\title{
Multiplexed Optical Fiber Sensors for Coal Fired Advanced Fossil Energy Systems
}

FINAL TECHNICAL REPORT

Report Period Start Date: October 1, 2008

Report Period End Date: $\quad$ December 31, 2011

Principal Investigator: $\quad$ Dr. Anbo Wang

Dr. Gary Pickrell

Report Issue Date: $\quad$ March 31, 2012

DOE Award Number: $\quad$ DE-NT0005591

Submitting Organization: Center for Photonics Technology

Virginia Tech

460 Turner St., Suite 303

Blacksburg, VA 24061 


\section{Disclaimer}

This report was prepared as an account of work sponsored by an agency of the United States Government. Neither the United States Government nor any agency thereof, nor any of their employees, makes any warranty, express or implied, or assumes any legal liability or responsibility for the accuracy, completeness, or usefulness of any information, apparatus, product, or process disclosed, or represents that its use would not infringe privately owned rights. Reference herein to any specific commercial product, process, or service by trade name, trademark, manufacturer, or otherwise does not necessarily constitute or imply its endorsement, recommendation, or favoring by the United States Government or any agency thereof. The views and opinions of authors expressed herein do not necessarily state or reflect those of the United States Government or any agency thereof. 


\begin{abstract}
This report summarizes technical progress on the program "Multiplexed Optical Fiber Sensors for Coal Fired Advanced Fossil Energy Systems" funded by the National Energy Technology Laboratory of the U.S. Department of Energy, and performed jointly by the Center for Photonics Technology of the Bradley Department of Electrical and Computer Engineering and the Department of Materials Science and Engineering at Virginia Tech.

This three-year project started on October 1, 2008. In the project, a fiber optical sensing system based on intrinsic Fabry-Perot Interferometer (IFPI) was developed for strain and temperature measurements for Ultra Supercritical boiler condition assessment. Investigations were focused on sensor design, fabrication, attachment techniques and novel materials for high temperature and strain measurements.

At the start of the project, the technical requirements for the sensing technology were determined together with our industrial partner Alstom Power. As is demonstrated in Chapter 4, all the technical requirements are successfully met. The success of the technology extended beyond laboratory test; its capability was further validated through the field test at DOE NETL, in which the sensors yielded distributed temperature mapping of a testing coupon installed in the turbine test rig. The measurement results agreed well with prior results generated with thermocouples.
\end{abstract}

In this project, significant improvements were made to the IFPI sensor technology by splicing condition optimization, transmission loss reduction, sensor signal demodulation and sensor system design. These innovations have yielded three journal publications and two conference papers.

The report is organized as follows. Chapter 1 and 2 reviewed the research background, which includes an introduction to the proposed sensing technology. Detailed system design, analysis and optimization are given in Chapter 3. The investigations for sensor fabrication, attachment techniques, and construction of the laboratory testing environment are summarized in Chapter 4, by the end of which the lab testing results, together with an in-depth analysis and discussion are provided. On November $16^{\text {th }}, 2011$, the developed sensing system was successfully deployed for field testing at DOE NETL. In Chapter 5, we reported on the technical details of the preparation and the result analysis for this field test. 


\section{List of figures and figure captions}

Figure 2-1 Schematic of proposed SMS-IFPI sensor .......................................................... 14

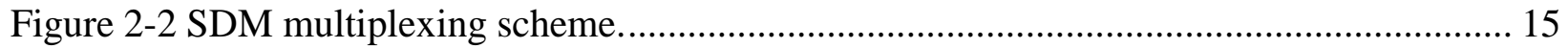

Figure 2-3 SDM multiplexing scheme with multiple channels. .......................................... 15

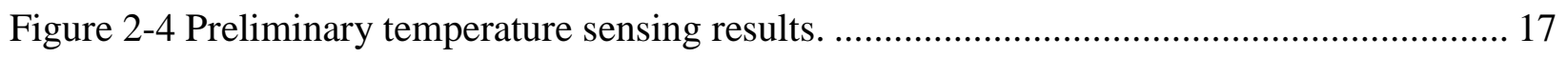

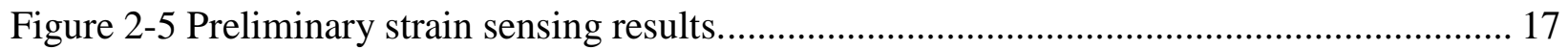

Figure 3-1 Computation procedure to calculate $m(\mathrm{z})$ for the index profile shown in (a). The $m$ profile, shown in (b), can be decomposed as a convolution of the functions shown in (c) and (d).

Figure 3-2. Relative reflectivity as a function of index profile transition width. Solid, dashed and dot-dash curves represent 1550,800 and $2300 \mathrm{~nm}$, respectively. ......................................... 21

Figure 3-3. Five lowest order mode profiles of MMF1. The fundamental mode profile of the excitation SMF is also plotted for comparison. All the modes are normalized, and SMF and MMF core boundaries are marked.

Figure 3-4. Light propagation in multimode fibers. (a) Field amplitude distribution in GI-MMF (MMF2). (b) Field amplitude distribution in SI-MMF, whose parameters are identical to MMF2.

Figure 3-5. Calculated and experimental results for the coupling loss and beam radius of the GIMMF. (a) Coupling loss variation as MMF length and (b) Beam radius variation as MMF length. For the simulation, the MMF1 fiber is selected. Corning InfiniCor600 was used for the

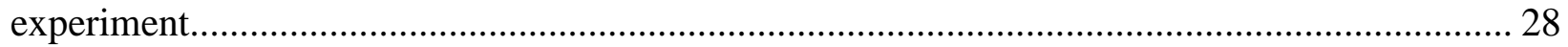

Figure 3-6. Experimental results showing the benefit of IFPI cavity length control. In (a), the FFT of the spectra for a link with (solid) and without (dashed) refocusing are compared. The relative OPD estimation error for each sensor in the links is plotted in (b) ............................. 31

Figure 3-7. Additional phase term for a two-mode cavity at different excitation ratios. ............. 34

Figure 3-8. Relationship between the total field vector and five individual mode vectors. ......... 35

Figure 3-9. Zoom-in view of the rotating vectors. Nine groups of vectors are plotted with equal angular spacing such that $v_{1}$ rotates exactly $2 \pi$. Dark lines: first group of vectors (beginning position); gray lines: last group of vectors (end position: $v_{1}$ coincides with $v_{1}$ in the first group, $v_{2}$ is a little behind $v_{2}$ in the first group; the effective vector $\Sigma$ is ahead of $\Sigma$ in the first group). Inset: global view of the vector rotation. Upper-left arrow: position of the first and last groups of vectors; other arrows illustrate the rotational direction of the vectors...................................... 36

Figure 3-10. Relative phase shift to $L P_{01}$ mode as $k$ increases. Plotted are phase shifts of $L P_{02}$

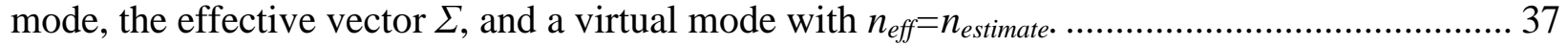

Figure 3-11. The evolution of the additional phase term $\theta$ with cavity length $L$. (a) The additional phase term in GI-MMF (MMF2). (b) The additional phase term in SI-MMF, whose parameters are identical to MMF2. 40

Figure 3-12. Verification of the analysis of the additional phase term $\theta$ in (26). The experimental demonstration is achieved by comparing the estimation of $\delta \mathrm{n}$ by two approaches for ten sensors multiplexed in a link. 41 
Figure 3-13. Output of a high-quality SMS-IFPI sensor under smoothly increasing temperature. When interrogated using traditional OPD techniques, the sensor experiences an abrupt jump in its demodulated signal.

Figure 3-14. (a) Slope $\kappa$ of the $O P D_{\mathrm{m}}{ }^{(1)}$ vs. $m$ curve as a function of phase estimation error $\Delta$ (b) An example of the tilting at a phase shift $\Delta \varphi \approx \pi$, given by the $O P D_{\mathrm{m}}{ }^{(1)}$ vs. $m$ curves for $n^{(1)}=27$ and $n^{(1)}=28$...... 46

Figure 3-15. OPD of an IFPI sensor (demodulated using traditional constant phase and new total phase algorithms) and its phase shift $\Delta \varphi$ under increasing temperature. Spectral data generated by computer simulation.

Figure 3-16. Performance of the varying total phase algorithm evaluated at different cavity lengths and SNR levels. MSE errors were calculated based on 1000 sampling points, among which phase shift from $0-1.2 \pi$ was evenly distributed. Each spectrum contains 20000 points spaced over 1520-1570nm..... 50

Figure 3-17. IFPI sensor OPD (demodulated using traditional constant phase and new total phase algorithms) and total phase $\Phi=k \cdot O P D+\varphi_{0}$ during an experimental controlled temperature increase. 51

Figure 3-18. IFPI strain sensor evaluation: demodulated by (a) traditional fixed-phase OPD method, (b) new varying total phase method.

Figure 3-19. Design of a sensing link with 22 IFPI sensors multiplexed with three different types of MMF. 62.5/125 MMF: Thorlab GIF-625; 50/125 MMF: Corning InfiniCor600; 100/140

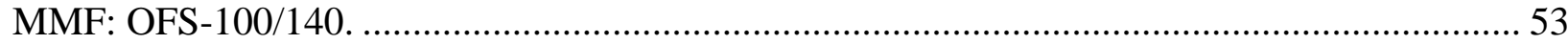

Figure 4-1. T-22 steel machined cut out (left) with dimensions seen at right. ......................... 55

Figure 4-2. Schematic of simulated testing environment ................................................ 57

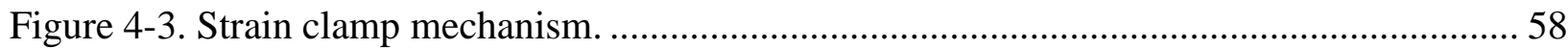

Figure 4-4. Strain sample and clamp mounting jig.......................................................... 59

Figure 4-5. Mounting jig during installation of strain sample............................................ 59

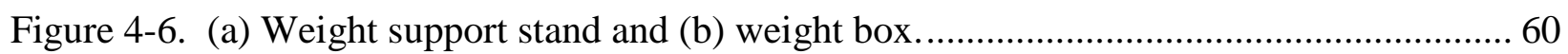

Figure 4-7. Complete assembly of simulated testing environment....................................... 61

Figure 4-8. A sensor response from a direct bonding attachment. The sensor failed around 350

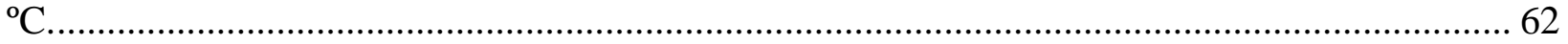

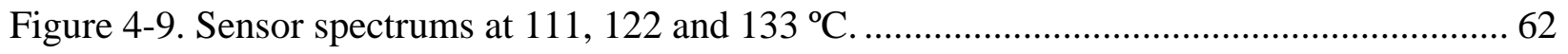

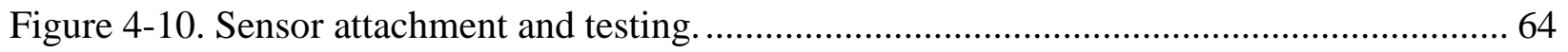

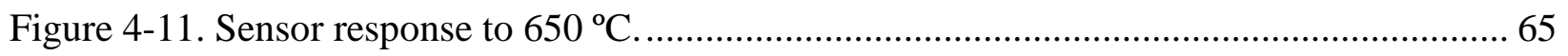

Figure 4-12. Sensor bonded to the T-23 tube by a set of mechanical brackets. ........................ 66

Figure 4-13.The compensation technique for reducing the strain in the sensor. ...................... 67

Figure 4-14. The sensor assembly when using in a harsh environment. .................................. 68

Figure 4-15. A drawing for temperature sensor protection tubes. ......................................... 69

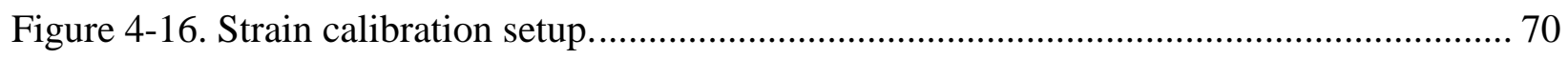

Figure 4-17. Comparison of simulated, experimental and theoretical variations in initial phase

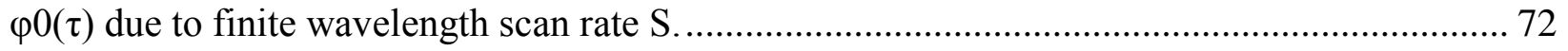


Figure 4-18. The signal processing approach shown in steps. (a) The original spectrum, with 10 sensors multiplexed. (b) The Fourier transform of the interpolated spectrum showing corresponding harmonics contributed by the sensors. The red dashed curve shows the digital bandpass filter applied to select one of the sensors. (c) The filtered signal, showing up as a pure sinusoid. (d) The tracked fringe peak locations plotted as a function of relative fringe order. Red solid line is the linear fitting.

$\ldots$

Figure 4-19 Temperature calibration curves of an IFPI link multiplexed with 6 sensors. ........... 76

Figure 4-20. Calculated histogram and corresponding distribution for sensor 1......................... 78

Figure 4-21. Strain calibration at different temperatures. Different slopes correspond to different strain-optic coefficient at various temperatures. The inset shows the calculated strain-optic coefficient at different temperatures. 79

Figure 4-22. Strain measurement with temperature compensation. (a) The OPD change measured with a strain sensor and a co-located temperature sensor as a function of temperature. (b) The

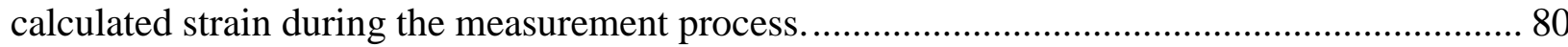

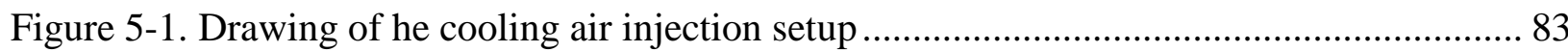

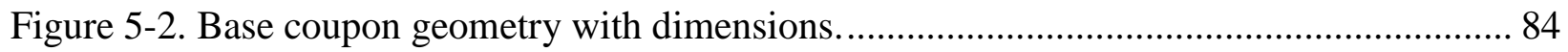

Figure 5-3. Fiber coating apparatus and the testing of the bending of the coated fiber. (a) Fiber coating setup: a tube furnace is aligned with a silica tubing in vertical position. On the bottom of the heating furnace is a glass chamber which contains the polyimide solution. The dip-coating process will be performed by firstly dipping the bare fiber inside the solution, and gradually pull it up through the furnace and the reshaping glass tube. (b) and (c): bending test of the coated fiber. The yellow thin piece in the white metal tubing is the coated fiber, and the whole piece was curled to a radius of 0.75 ", and heated. (b) is a zoomed-in view of (c)..................................... 85 Figure 5-4. Nonuniform polyimide coating (lower-right) compared to commercial acylate coated

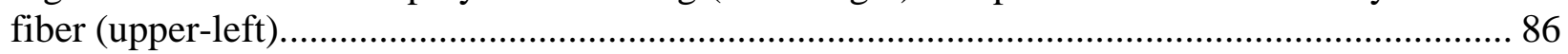

Figure 5-5. Illustration of the sensor chain fabrication process. SMF: single-mode fiber; MMF:

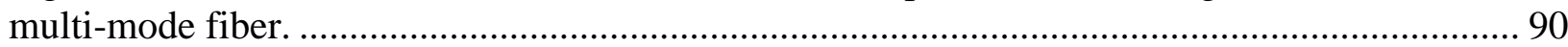

Figure 5-6. Chain characterization results. (a) and (b) show the spectrum obtained by CTS. (c) and (d) show the Fourier Transform of the spectra in (a) and (b). The figures represent a change in down-link signal quality after chain installation, as reflected by the decreased harmonic intensities. 91

Figure 5-7. Calculated chirp intensities of the sensors multiplexed in one link. The cavity lengths are (a) $1040 \mu \mathrm{m}$, (b) $1560 \mu \mathrm{m}$, (c) $2080 \mu \mathrm{m}$ and (d) $2600 \mu \mathrm{m}$. Colors represent different installation steps. 92

Figure 5-8. The calibration curve (dOPD/OPD) against dT determined with high accuracy for the sensor chain used in the field test. 95

Figure 5-9. (a) Mechanical drawing of the testing environmental chamber design. (b) the measured temperature using an installed sensor chain during the testing process. ....................... 96 Figure 5-10. (a) Design of the optical fiber feedthrough assembly. (b) Feedthrough assembled in the lab. 97

Figure 5-11. (a) Design of the 1" O.D. tube with the fitting plug. The plug provides stabilization of the metal tubing, and the bend-insensitive fiber will be curled inside the tubing. (b) Cross- 
sectional view showing the machined plug. The central bore is for the fiber to go through, while the surrounding holes provide air-path to maintain balanced pressure on both sides of the plug. 98 Figure 5-12. Photo of the installed fiber optic probe (photo taken during the field test). ........... 99 Figure 5-13. Measured temperature (real time) with direct OPD demodulation algorithm. ..... 101 Figure 5-14. Post-processed data with the total phase algorithm. The control parameters are plotted with the same time scale for better comparison................................................. 102

Table 3.1. Splicing Parameters for SMS-IFPI Sensor ........................................................ 21

Table 3.2. Fiber Parameters Used for Simulation.................................................................. 26

Table 3.3. Power Coupling Coefficients of Five MMF Modes............................................... 26

Table 3.4. Simulation Parameters for SMS-IFPI Sensor. ........................................................ 49

Table 3.5. Key Properties of Three Types of MMF. ............................................................. 53

Table 4.1. Temperature calibration coefficients. ........................................................... 77

Table 4.2. Temperature measurement accuracy. ............................................................... 77

Table 4.3. Measured temperature resolution of the sensors....................................................... 78

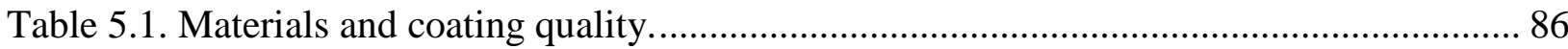

Table 5.2. Summary of investigated coupon configurations. ................................................ 88

Table 5.3. Sensor fabrication procedure ........................................................................ 89

Table 5.4. Parameters affecting the coupon temperature during the field test. ........................ 100 


\section{CONTENTS}

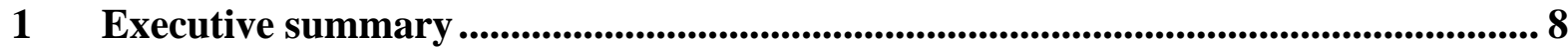

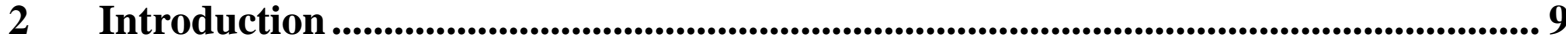

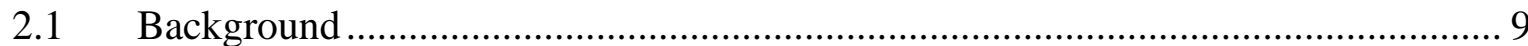

2.2 Technology review................................................................................... 10

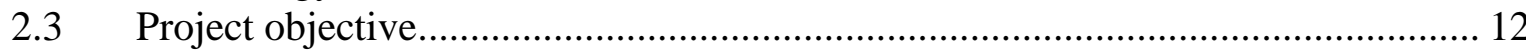

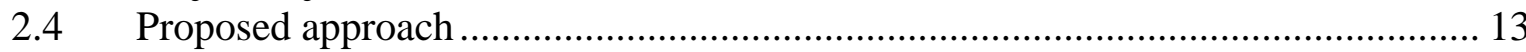

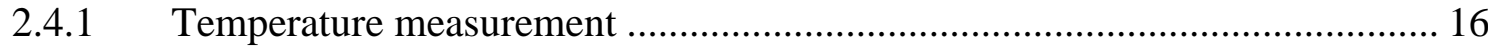

2.4.2 Strain measurement ........................................................................ 17

3 Sensor design and optimization ........................................................................................ 18

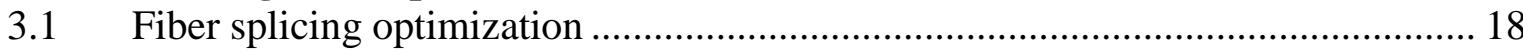

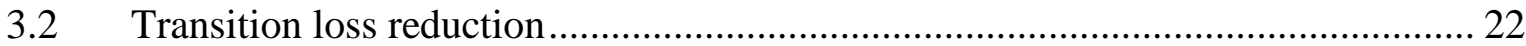

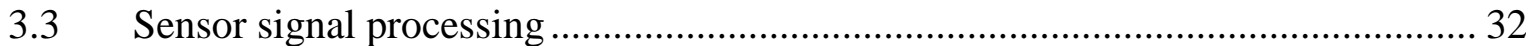

3.3.1 Modeling of the IFPI structure and the introduction of the additional phase.. 32

3.3.2 Sensor signal processing and the total phase approach .............................. 42

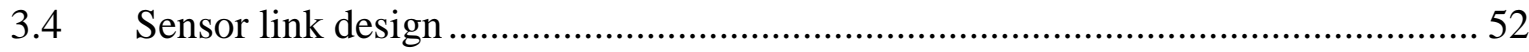

$4 \quad$ Laboratory testing of sensors ................................................................................... 54

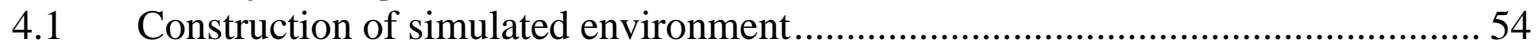

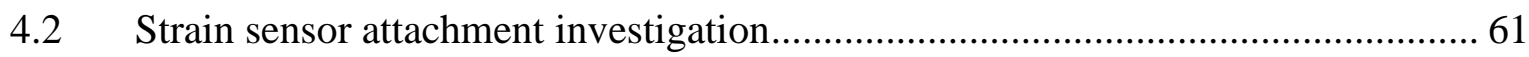

4.2.1 Sensor response using the direct bonding technique ................................. 61

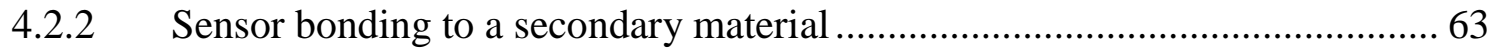

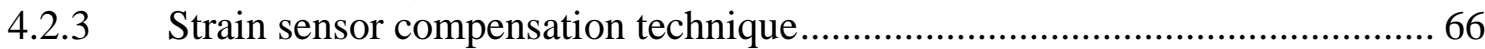

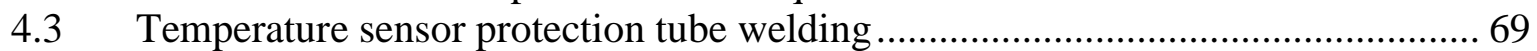

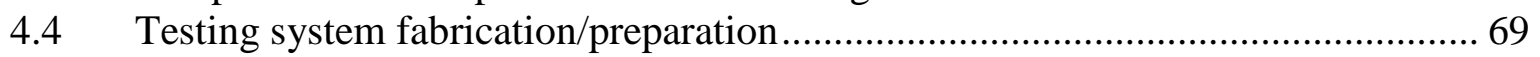

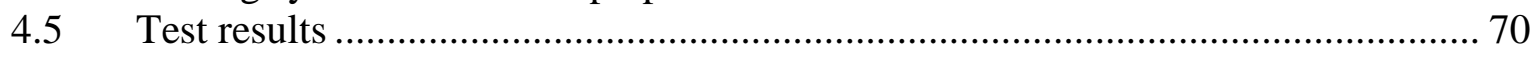

4.5.1 Some signal processing remarks.......................................................... 70

4.5.2 Temperature calibration and measurement results ................................... 75

4.5.3 Strain calibration and measurement results ............................................. 78

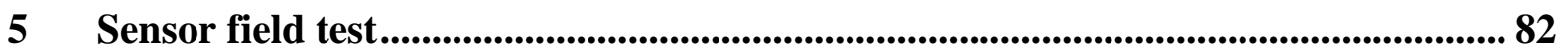

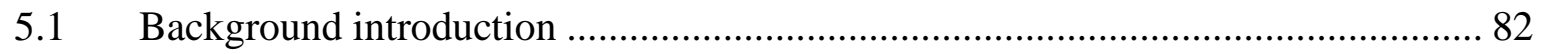

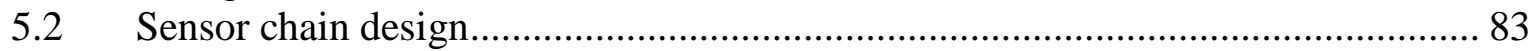

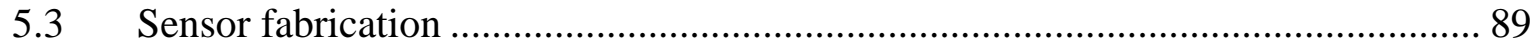

5.4 Sensor performance characterization ............................................................. 90

5.5 Simulated environmental test................................................................... 95

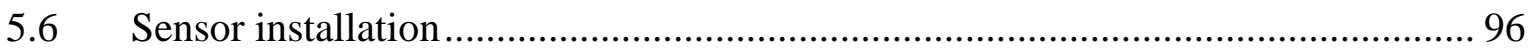

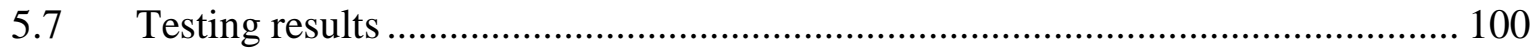

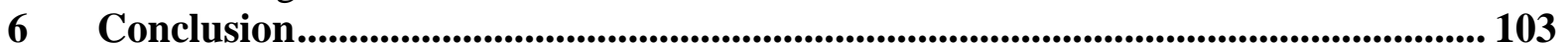

$7 \quad$ Reference..................................................................................................................... 104

Appendix i. Matlab code for OPD demodulation .................................................................... 106

Appendix ii. Alstom Power technical report .......................................................................... 108 


\section{EXECUTIVE SUMMARY}

The objective of this project is to develop a fiber optic (FO) strain, and temperature sensor technology for the needed Ultra Supercritical (USC) boilers Condition Assessment performance required by the next generation of Fossil Energy Power Systems. These new sensors will not only be able to operate at temperatures exceeding the current sensor capabilities, but also have the desired multiplexed capability for a distributed sensor network. It is this larger component coverage that will enable predictive Condition Assessment models of the USC process to more accurately identify any operating conditions which will adversely impact boiler reliability and hence plant availability. Currently there are no distributed sensor networks rated for in-furnace conditions associated with a USC boiler. This project targets the design, development and testing of a candidate FO sensor technology which will provide vital information on USC boiler component conditions.

Throughout the project timeline, research investigations have been emphasized on fabrication of low insertion-loss and high dynamic range sensors, sensor attachment and packaging technologies, the construction of the simulated testing environments, cascade capabilities of network sensors and sensor capabilities of handling sharp bending and high temperatures. Fiberoptic sensing technologies using intrinsic Fabry-Perot interferometric (IFPI) sensors have been developed and demonstrated for strain and temperature measurements. A network sensor link containing six sensors (five temperature sensors and one strain sensor) has been developed and tested for waterwall cooling pipes for the Ultra Supercritical (USC) boilers at Alstom power Inc. And a network sensor link containing four temperature sensors has also been developed and tested for the temperature distributions on a cooling coupon of the turbine at National Energy Technology Lab (NETL). 


\section{INTRODUCTION}

\subsection{Background}

The next generation of fossil-fueled boilers has, as part of a major $\mathrm{CO}_{2}$ mitigation strategy, the goal of higher energy conversion efficiency. Ultra Super Critical (USC) steam cycle designs have an efficiency target in the range of $45-47 \%$. This is an increase in efficiency of $10 \%$ over current saturated designs and results in a $\mathrm{CO}_{2}$ decrease of $25-30 \%$ compared with current technology. This higher boiler cycle efficiency has a similar impact on all boiler emissions. Increased efficiency is a clear path forward for the evolutionary "clean coal" power station.

In addition to higher efficiencies, the need to maintain plant availability is critical to taking advantage of these USC efficiency improvements. The need to operate the plant to improve operating performance and stay within equipment or material limits and other constraints is key. The utilization of advanced sensing and controls technology to affect such improvements in plant availability is important to these future plants.

This movement toward USC and the design targets of $760{ }^{\circ} \mathrm{C}$ steam temperature and 5000 psi pressure places new challenges on all of the materials that would be utilized in an USC design. Boiler components are becoming increasingly monitored for the purpose of predicting the amount of remaining life. These component Condition Assessment (CA) models all require process sensor measurements in order to produce reliable predictions. For a USC boiler operating under these higher temperatures and pressures there is a need for local measurements rather than the global process operation and control measurements available from the current generation of sensors. The local measurements are needed to assess equipment health or impending problems over and along the entire components area.

A USC boilers CA requires that new extreme environment sensors technology be developed. USC boiler conditions $\left(760^{\circ} \mathrm{C} 5000\right.$ psi steam side higher gas side) lead to accelerated

degradation. In order to capture the higher efficiency of the USC steam cycle unit, availability must remain high. What is required is that new sensors must perform in the boiler with the same or better accuracy and long term operating reliability as the lower temperature versions. 


\subsection{Technology review}

Temperature can be defined in terms of the heat transferred in a Carnot cycle. This is generally not the most practical way to measure temperature, and in practice many different techniques are used depending on the temperature measurement requirements. Theoretically speaking, all temperature measurements are indirect; every temperature measurement involves the use of some type of calibrated transducer to convert a measurable quantity into a temperature value. Those transducers are the parts of the temperature sensors that convert changes in temperature into other measurable physical quantities, such as volumetric expansion (liquid-filled thermometer), dimensional change (bimetallic thermometer), EMF electromotive force (thermocouple), resistance (resistance temperature detector, or RTD), radiated energy (radiation thermometer), or some other characteristic of a material that varies predictably and reproducibly with temperature. However, in industrial process measurement and control, the concept of direct and indirect temperature measurement has a different meaning. A direct measurement is a measurement of the temperature of the product itself. An indirect measurement is a measurement of some other parameter from which one can infer the product temperature.

Temperature may be measured using any physical system with an extrinsic property related to its temperature. The overwhelming majority of temperature measurements are made using only a few basic types of instruments: liquid-in-glass thermometers, thermocouples, resistance thermometers and radiation thermometers. These conventional and traditional measurement instruments have been in use for several decades, and the major sources of instability or drift, as well as possible systematic errors, are well understood. Further, there is extensive industrial experience with these techniques, which allows the user to design a reliable system with a high chance of success. These traditional techniques also offer the advantage of having a large range of suppliers offering equivalent plug-compatible equipment in product ranges, product support and replacements are unlikely to be a problem. As mature technologies, they are also generally much cheaper.

There is a continuing stream of new instrumentation techniques entering the market due to the increased availability of technologies as a spin-off from the electronics and computer industries. Advances in communications technologies have made laser sources and optical fibers 
cheap, reliable and readily available. Many of the unconventional thermometry techniques require complex processing of the signals received from the sensor. The availability of cheap computers integrated into the measurement equipment enables the often complex processing associated with the more unusual sensors to be made available to the user.

These newer techniques may offer the promise of doing a much better job than traditional techniques. Also, generally speaking, these "unconventional" techniques in thermometry have been developed in response to a specific measurement problem for which the commonly used traditional methods are not suitable. Those specific measurement requirements have become quite common in today's modern industrial environments, such as low thermal mass sensors for fast time response, gas temperature measurement, measurement of internal temperature profiles, measurements in hostile environments, e.g., EMI (Electromagnetic interference), radiation, corrosion and intrinsic safety, where the measurement environment should not be affected by measurement units, optical sensors are easily able to satisfy these requirements.

A thermocouple is an assembly of two wires of unlike metals joined at one end, the hot end, and at the other end, the cold junction, across which the open circuit voltage is measured. This voltage (electromotive force, or EMF), also called Seebeck voltage, depends on the difference in temperature between the hot and the cold junction and the Seebeck coefficient of the two metals. The Seebeck EMF develops when there are differences in temperature between junctions of dissimilar metals in the same circuit. Also known as the thermoelectric effect, German physicist J.T. Seebeck discovered the phenomenon in 1821. A measurement indicates the temperature of the hot junction when one knows the cold junction temperature or when measurement circuitry compensates for cold junction temperature.

From the nature of the proposed measurements, accurate temperature and strain measurements are required at the specified locations of industrial applications. Especially, surface and multiple-point temperature measurements are required during the heating and cooling operations of the boiler and turbine. Therefore, well-suited sensor attachment and cascade techniques are to be developed for the sensors. Fiber-optic sensors have superior advantages for bonding and embedded into structures because of their small size, high accuracy, long lifetime, immunity to EM interference and the ability to be multiplexed, etc. Furthermore, 
they can be cascaded into a network containing multiple sensors sharing one light source and detection system with minimum insertion loss and maximum signal dynamic range. In conclusion, fiber-optic sensors have great advantages over traditional sensors and will be utilized and developed for this project.

\subsection{Project objective}

The objectives of this project include: 1) the development of a multiplexed fiber optic sensor system for the measurement of strain and temperature for conditions expected in the next generation of high efficiency Ultra Supercritical (USC) boilers; 2) demonstration of sensor network performance and survivability when exposed to the simulated conditions of an USC Boiler; and 3) development and testing of a sensor network when attached to the coupon surface of the turbine cooling jet for temperature measurements.

The scope of work entails an analysis of advanced combustion processes, including USC boilers, to identify the measurement requirements that are applicable to fiber optic sensors. Sensor development efforts include the maturation of intrinsic Fabry-Perot interferometer (IFPI) sensor design for measurement of temperature, and strain. The proposed IFPI sensor is based on a single-mode-multimode-single-mode (SMS) structure, which has been preliminarily demonstrated. These sensors will also be multiplexed onto a single fiber and sets of fibers for the development of a sensor network. The sensors will be packaged for UCS conditions and a demonstration of the sensor network will be made.

Given the project's interdisciplinary nature, effective communication and coordination between the team members will be crucial for the technical tasks in order to be completed successfully. The sensor development team and the packaging/protection team both at Virginia Tech will hold regular meetings to ensure the packaged sensors can deliver the required performance.

Project management tasks include the timely preparation and submission of reports (i.e. Quarterly Progress Reports, Final Reports, etc.) and frequent communication and coordination between DOE NETL and the project participants. 
Technical requirements for real time measurement and monitoring of the strain along the tubes, pipe welds and vessel headers, as well as temperatures within the next generation of advanced boiler systems will be defined. This will be performed using plant designs and knowledge of plant operations available to the development team.

Important parameters such as operational temperatures, dynamic range, resolution, accuracy, frequency response, maximum acceptable sensor size, distance between sensor elements, minimum distance to lead the sensor signal out of the hot zone, and appropriate atmospheres in these boiler industries will be determined. Sensor requirements will be selected such that the defined next generation boilers (SC and USC) will be represented by the simulated testing. Based on the specified requirement metrics, a matrix of measurement need and the sensor performance will be prepared.

\subsection{Proposed approach}

Intrinsic Fabry-Perot (FP) interferometric (IFPI) fiber sensors have attracted considerable interest for measuring temperature, strain, pressure and many other physical parameters in the past decade. IFPI sensors possess all the advantages of FP-based fiber sensors such as high spatial resolution, high accuracy, high measurement resolution, harsh environment capability, and electromagnetic immunity. In addition, IFPI sensors have a unique advantage of small size. When used for temperature and strain measurement, the sensing element is simply a section of optical fiber without the need for external transducers. Therefore, the diameter of a packaged sensor can be as small as a few hundred micrometers. Traditionally IFPI sensors are made by thermally fusing section of fiber with mirrors coated on both ends in between two single-mode fibers (SMFs). The methods used to produce the mirrors include vacuum deposition, magnetron sputtering, or e-beam evaporation $[1,2,3]$. The cost of the sensors is prohibitively high due to the expensive sensor fabrication methods. In addition, the sensors have high reflection losses induced by the mirrors, leading to a limited multiplexing capability. 


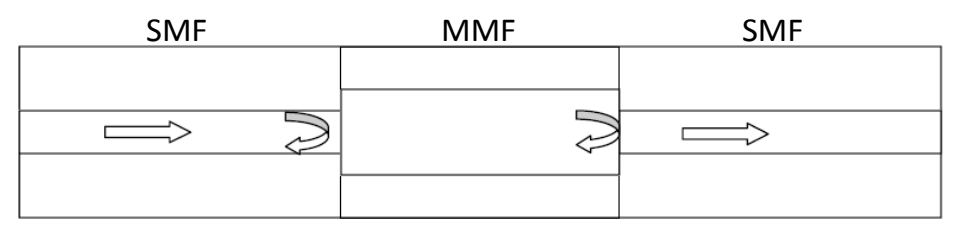

Figure 2-1 Schematic of proposed SMS-IFPI sensor

We recently proposed and demonstrated the temperature and strain measurement applications of an IFPI fiber sensor that does not require thin-film mirror. [4] The proposed IFPI sensor is based on a single-mode-multimode-single-mode (SMS) structure, as shown in Figure 2-1. The sensor is fabricated by simply splicing a section of multimode fiber (MMF) in between two SMFs. Because the core refractive indices of the SMF and the MMF are slightly different, a weak Fresnel reflection is generated by the two splicing points and thus an IFPI sensor is formed.

The optical path distance (OPD) between the two reflections is

$$
L=2 * n * l,
$$

where $l$ is the length and $n$ is the refractive index of the fiber between the two single-modemultimode fiber junctions. Due to the low reflectance at each point, the interference between the two reflections can be approximated by

$$
I(\lambda)=I_{1}(\lambda)+I_{2}(\lambda)+2 \sqrt{I_{1}(\lambda) I_{2}(\lambda)} \cos \left(\frac{2 \pi L}{\lambda}+\phi\right),
$$

where $I(\lambda)$ is the optical power measured at wavelength $\lambda, I_{1}(\lambda)$ and $I_{2}(\lambda)$ are reflected optical power from the two junctions at wavelength $\lambda$, and $\lambda$ is the wavelength of the light source.

From Eqs (2.1) and (2.2), it can be seen that that the IFPI sensor can be used to measure any quantity that can change either the refractive index or fiber dimension or both. Some common parameters include pressure, temperature and strain. Of course, it is important for each application that the sensor is purposely designed to have an enhanced sensitivity to the measurands and least sensitivity to other parameters. Also, since multiple such sensors are to be arranged close to each other and if they are designed to have different responses to various 
quantities, simultaneous measurement of multiple quantities can be realized. The same function can also provide self compensation if only one quantity is of interest.

It is clear that compared to traditional IFPI sensors, the proposed SMS-IPFI sensors are much easier and cheaper to fabricate, and have a high yield-rate. In addition, due to the weak reflection at the two splicing points, a large number of sensors can potentially be multiplexed to achieve distributed measurement.

As described above, the small reflection loss of each of the proposed IFPI sensor renders the possibility of high multiplexing capability. Many multiplexing techniques for interferometric sensors have been investigated, including time and wavelength [5, 6], code [7], spatial [8], and coherence [9] division multiplexing. For the demonstrated IFPI sensors, the best multiplexing method is spectrum division multiplexing (SDM).

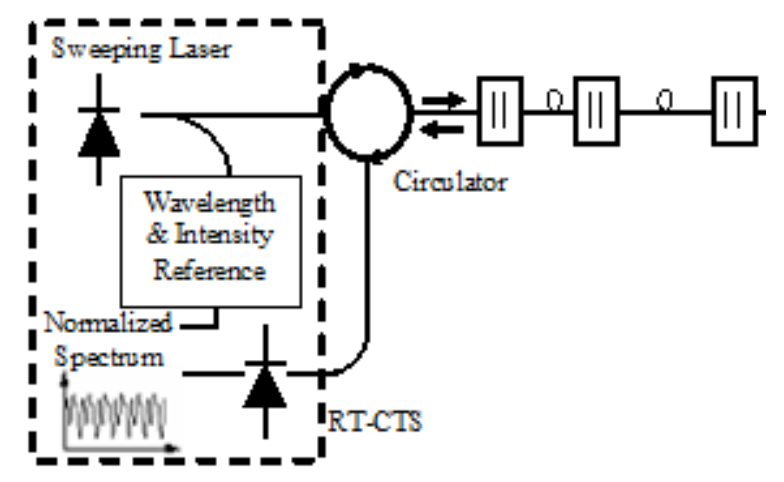

Figure 2-2 SDM multiplexing scheme.

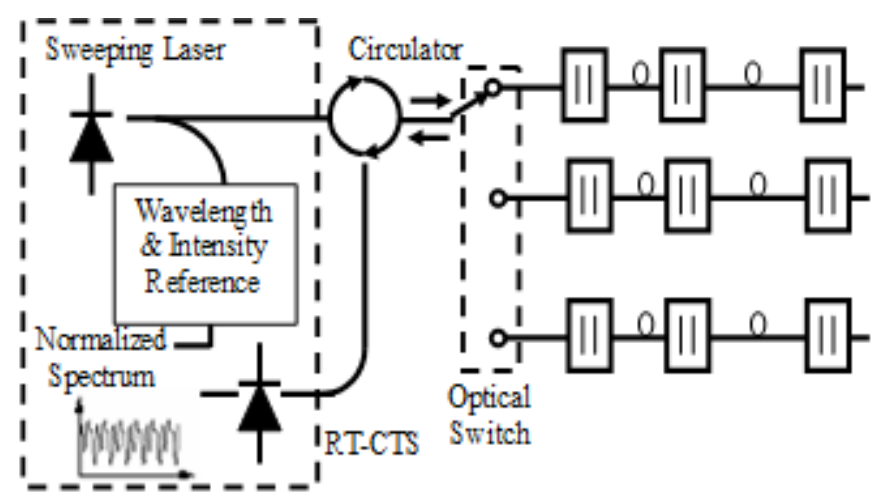

Figure 2-3 SDM multiplexing scheme with multiple channels. 
In the SDM scheme as shown in Figure 2-2, the sensors are sequentially arranged and signals from different sensors are multiplexed in the frequency domain using a wavelength sweeping laser or a broadband source. Continuous light is transmitted down the sensor array. If the cavity length of each sensor is different from others, the interferometric fringe pattern of each sensor will yield a unique spatial frequency in the Fourier transformed spectrum. By separating each frequency component and measuring its frequency and phase information, the optical path length of each IFPI sensor can then be determined.

The multiplexing capability can be further enhanced by a multi-channel instrumentation, as shown in Figure 2-3. In this scheme, a $1 \times \mathrm{M}$ optical switch is inserted between the optical circulator and the sensing fiber. The optical switch can be electronically controlled. Each of the $M$ channels of the optical switch can connect a sensing fiber with multiple sequentially arranged sensors and all the channels can share the same optical demodulation system.

\subsubsection{Temperature measurement}

The optical path distance (OPD) of the IFPI sensor will change due to the temperature dependence of the refractive index and thermal expansion. The change of the OPD is given by

$$
\delta L=2 *(\delta n * l+n * \delta l)=2 *\left(n_{T} * \Delta T * l+n * l * \alpha * \Delta T\right),
$$

where $n_{T}$ is thermal optic coefficient $\left(\sim 1.2 * 10^{-5} /{ }^{\circ} \mathrm{C}\right)$ and $\alpha$ is the thermal expansion coefficient $\left(\sim 0.5^{*} 10^{-6} /{ }^{\circ} \mathrm{C}\right)$. The feasibility of the IFPI for temperature sensor has been experimentally confirmed. Figure 2-4 shows the temperature response of an initial IFPI temperature sensor with a $408 \mu \mathrm{m}$ cavity length tested in the temperature range $25-600^{\circ} \mathrm{C}$ with a resolution of $0.1^{\circ} \mathrm{C}$. 


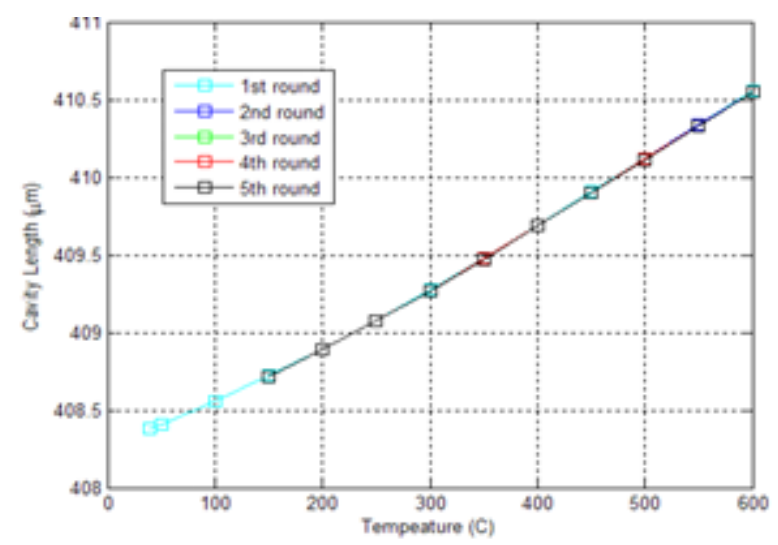

Figure 2-4 Preliminary temperature sensing results.

\subsubsection{Strain measurement}

The IFPI sensor can also be designed to measure strain. The OPD of the IFPI sensor will change due to the deformation of the cavity and the stress-optic effect of the optical fiber. The change of the OPD is given by

$$
\delta L=2 *(\delta n * l+n * \delta l)=2 *\left(n_{c} * \varepsilon * l+n * \varepsilon * l\right),
$$

where $\mathrm{n}_{\mathrm{c}}$ is a coefficient related to stress-optic effect and $\varepsilon$ is the fiber strain defined as $\delta l /$. Figure 2-5 shows preliminary test results of an IFPI strain sensor as a function of applied tensile stress.

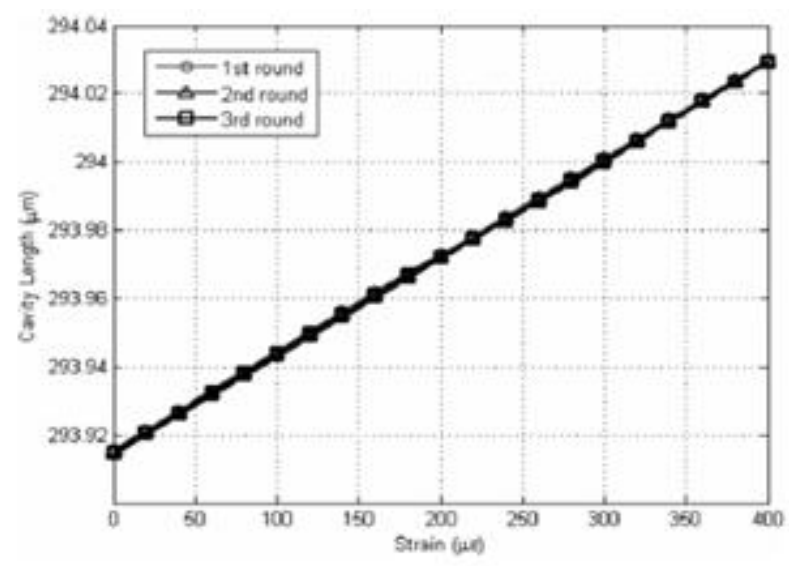

Figure 2-5 Preliminary strain sensing results. 


\section{SENSOR DESIGN AND OPTIMIZATION}

In this section the detailed design merits of the IFPI sensor and sensing systems will be given. We will first start with our analysis of the optimization of the fiber splicing condition seeking a balanced performance in terms of sensor signal to noise ratio (SNR) and mechanical strength. In order for the desired multiplexibility, we will also investigate light refocusing within the multimode fiber (MMF) cavity, which is of great significance in reducing the insertion loss associated with the sensor. We will also expound on some practical signal demodulation issues that affect the demodulation accuracy and resolution of the sensing system. We will finally conclude, based on the above technical discussions, an optimized sensing system design with which experiments were conducted for the ensuing chapters.

\subsection{Fiber splicing optimization}

The sensor is basically a low-finesse fiber optic (FO) Fabry-Perot (FP) interferometer measuring the optical path difference (OPD) of the FP cavity (see Section 2). The FP-type reflections are generated by the weakly reflective boundaries formed by the fiber splices between two types of fibers. The Fresnel reflection created by the refractive indices differences between the fiber cores will support reflections generating the interference pattern upon which the OPD is calculated. As compared to traditional low-finesse FO FP cavities, the reflectivity for this IFPI structure is in principle, ultra-weak, as determined by the Fresnel reflection coefficient

$$
R=\frac{\left(n_{1}-n_{2}\right)^{2}}{\left(n_{1}+n_{2}\right)^{2}}
$$

in which the refractive indices of the cores of the neighboring fibers are $n_{1}$ and $n_{2}$, such that $n_{1}-n_{2}$ represents their difference. For typical fibers employed in forming the sensor, the SMF lead in fiber has a relative index difference of $0.3 \%$ and the MMF has a relative index difference of $1 \%$, upon which we can estimate the value of Eq. 3.1 accurately as $\mathrm{R}<-49 \mathrm{~dB}$. The upper bound is reached once the transition width of the fiber splicing is approaching zero. However, in real practices, splicing of fibers results in a finitely valued transition width which would, as will be shown in the following, significantly reduce the reflectivity as predicted in Eq. 3.1. 
During a real fusion splice process, the extremely high temperature and finite arc duration give rise to diffusion of the dopant across the splicing plane, which tends to smear any index difference across the boundary, thus reducing reflectivity. In order to model the complex index profile, numerical methods have been used [10]. Here we consider a simple analytical solution to show how the reflectivity decreases with widening index transition length.

Suppose a complex index distribution along the fiber axis $n(\mathrm{z})$, and assume the index is uniform on the fiber cross section for any given $\mathrm{z}$. For the above index profile, at location $\mathrm{z}$ the amplitude reflectivity is given by (1). Assuming the total change of $n$ is small, $r(\mathrm{z})=n^{\prime}(\mathrm{z})$ $\mathrm{dz} / 2 n(\mathrm{z})$, where $n^{\prime}(\mathrm{z})=\mathrm{d} n / \mathrm{dz}$ is the changing rate of $n$ with respect to $z$. Let $\mathrm{z}=0$ represent the point at which the total reflected field is detected, and the field reflected at $\mathrm{z}=\mathrm{z}_{0}$ will pick up a phase term $\exp \left[-j 2 \beta \mathrm{z}_{0}\right]$ at $\mathrm{z}=0$. The term $\beta$ denotes the optical propagation constant in the fiber (here for simplicity the fiber is assumed to be single-mode). The total field reflectivity, defined by the ratio between the reflected field and the input field at $\mathrm{z}=\mathrm{z}_{0}$ can thus be expressed as

$$
r_{t o t}=\int_{-\infty}^{\infty} m(z) e^{-j(2 \beta) z} d z
$$

where $m(z)=n^{\prime}(z) / 2 n(z)$ and the power reflectivity is found by $R=\left|r_{\text {tot }}\right|^{2}$. Equation 3.2 shows the Fourier transform (FT) relationship between $m(\mathrm{z})$ and $r_{\text {tot }}$. It should be noted that such mathematical relationship has been used for frequency domain distributed sensing [11]. The reflection spectrum of an IFPI with finite index transition length is modeled by applying Eq. 3.2 and some basic properties of FT.

The splice point with diffusion-induced finite transition width is modeled by a half-period cosine function, and the index profile along the z-axis of an imperfect IFPI is shown in Figure 3-1 (a). The FP cavity length is $L$, the transition width is $\Delta$, and the total index change is $\delta n$. The corresponding $m(\mathrm{z})$ distribution is calculated and shown in Figure 3-1 (b). This distributed reflectivity profile can be represented as the convolution of two functions $\left(f_{1} * f_{2}\right)(\mathrm{z})$, where $f_{1}$ is a rectangular-windowed cosine (Figure 3-1 (c)), and $f_{2}$ is formed by two Dirac delta functions at $\pm L / 2$ with amplitude (-/+) unity (Figure 3-1 (d)). The above procedure assumes a constant $n$ in the denominator of $m$, which is reasonably accurate for small index changes. The final power spectrum, by using Eq. 3.2 and some mathematical manipulations, is 


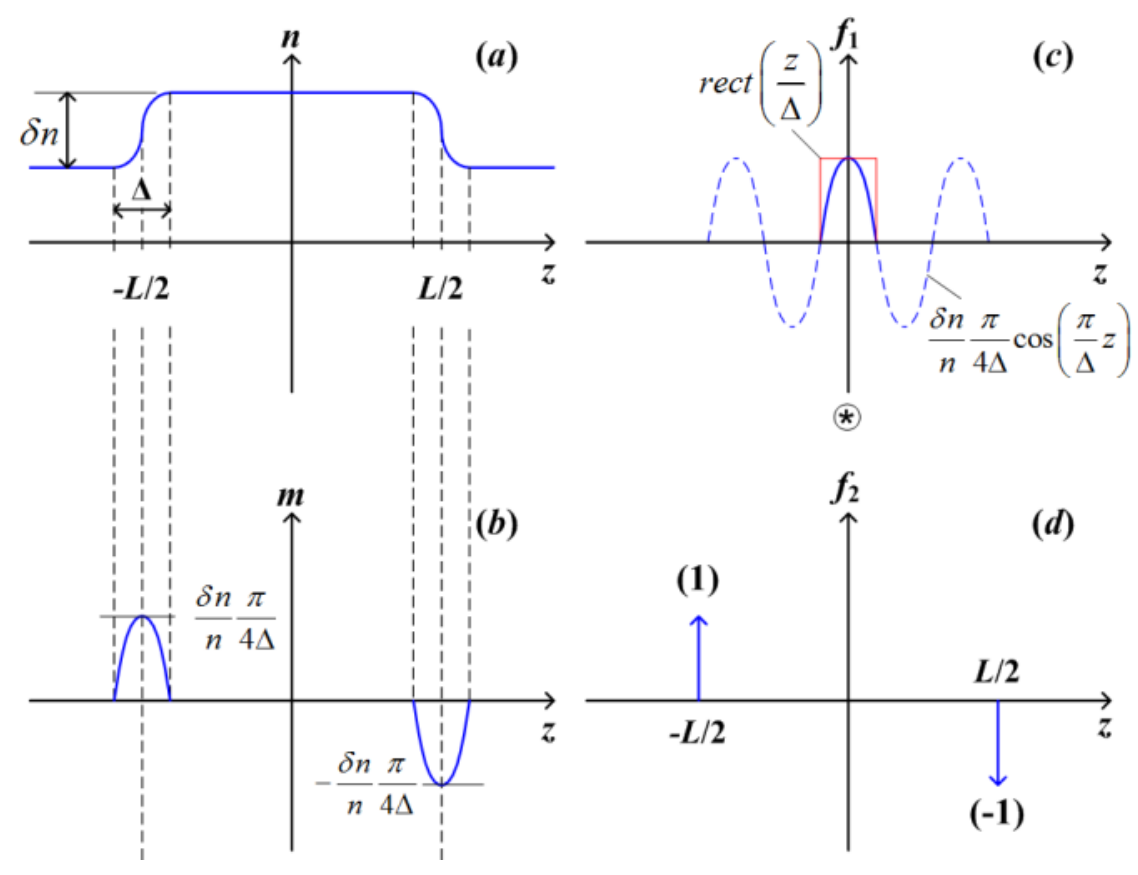

Figure 3-1 Computation procedure to calculate $m(z)$ for the index profile shown in (a). The $m$ profile, shown in (b), can be decomposed as a convolution of the functions shown in (c) and (d).

$$
I=\frac{\pi^{2}}{2}\left(\frac{\delta n}{4 n}\right)^{2} \Gamma^{2}(\beta \Delta)[1-\cos (2 \beta L)]
$$

where $\Gamma(x)=\operatorname{sinc}(x-\pi / 2)+\operatorname{sinc}(x+\pi / 2)$ and $\operatorname{sinc}(x)=\sin (x) / x$. The result is shown in Figure 3-2, in which the relative reflectivity (reflectivity normalized to the maximum reflectivity defined in (1)) is plotted as a function of the transition width $\Delta$ for three different wavelengths. $n$ is assumed to be 1.46. When the transition length approaches $\lambda / 2$ ( $\lambda$ being the vacuum wavelength), the reflectivity drops more than $20 \mathrm{~dB}$ from its maximum value. 
In practice, the optimal splicing condition is found by compromising splicing strength and the reflectivity. In Table 3.1, two splicing conditions are compared (with fusion splicer Sumitomo Type-36): a standard MMF-MMF splice setting ("hot"), and an optimized splicing condition for IFPI fabrication ("cold"). Splicing was performed using SMF (SMF-28, Corning Inc.) and GI-MMF (GIF625, Thorlabs, Inc.).

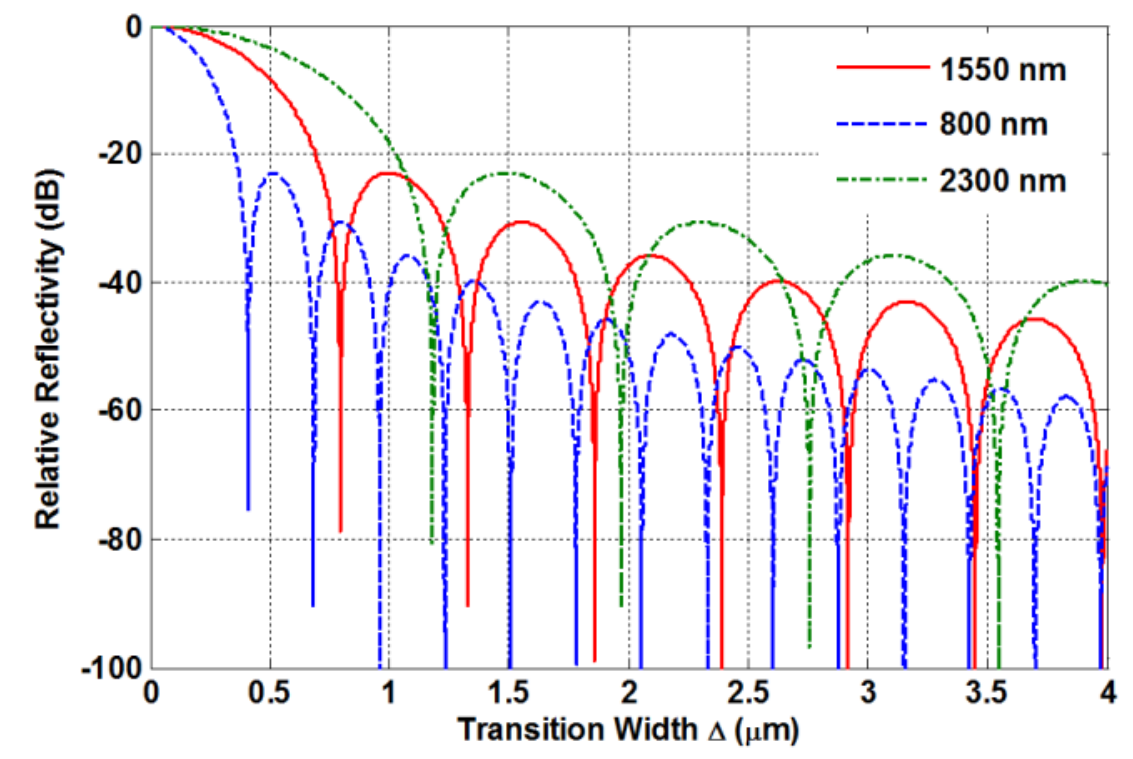

Figure 3-2. Relative reflectivity as a function of index profile transition width. Solid, dashed and dotdash curves represent 1550,800 and $2300 \mathrm{~nm}$, respectively.

Table 3.1. Splicing Parameters for SMS-IFPI Sensor

\begin{tabular}{|c|c|c|c|c|c|c|c|}
\hline Type & $\begin{array}{l}\text { Duration } \\
\text { (second) }\end{array}$ & $\begin{array}{l}\begin{array}{l}\text { Prefusion } \\
\text { (second) }\end{array} \\
\end{array}$ & $\begin{array}{l}\text { Gap } \\
(\mu \mathrm{m})\end{array}$ & $\begin{array}{l}\text { Overlap } \\
(\mu \mathrm{m})\end{array}$ & $\begin{array}{l}\text { Power } \\
\text { (level) }\end{array}$ & $\begin{array}{l}\text { Strength }^{2} \\
(\mu \varepsilon)\end{array}$ & $\begin{array}{l}\text { Reflectivity } \\
\text { (dB) }\end{array}$ \\
\hline Hot & 4.5 & 0.1 & 10 & 15 & 20 & 9,800 & $<-70$ \\
\hline Cold & 0.5 & 0.2 & 5 & 10 & 22 & 6,700 & -50 \\
\hline
\end{tabular}




\subsection{Transition loss reduction}

This subsection provides a detailed analysis of GI-MMF cavity refocusing. The theoretical analysis begins with the traditional ray matrix method, and is further extended to the more rigorous fiber modal analysis. Both methods are shown to give similar results.

The ray matrix approach adopts the treatment by Emkey and Jack [12]. The modeling process begins with the complex $q$ parameter of a Gaussian beam with curvature radius $R_{\mathrm{C}}(\mathrm{z})$ and beam radius $\omega(\mathrm{z})$

$$
\frac{1}{q(z)}=\frac{1}{R_{C}(z)}-i \frac{\lambda}{\pi \omega^{2}(z) n}
$$

The $q$ parameter at the SMF-MMF boundary is defined as $q_{1}$, and the input field is the $\mathrm{LP}_{01}$ mode of the SMF. The planar wavefront at the $\mathrm{z}=0$ splice interface requires $R_{\mathrm{C}}(0) \rightarrow \infty$. In order to calculate the $q$ parameter in the MMF at $\mathrm{z}=l$, the transformation is readily obtained by

$$
q_{2}=\left(A q_{1}+B\right) /\left(C q_{1}+D\right)
$$

the elements $A, B, C$ and $D$ are determined by the GI-MMF through

$$
\left[\begin{array}{ll}
A & B \\
C & D
\end{array}\right]=\left[\begin{array}{cc}
\cos (g l) & \frac{1}{g} \sin (g l) \\
-g \sin (g l) & \cos (g l)
\end{array}\right]
$$

where the $g$ parameter is defined as

$$
g=\frac{\sqrt{\delta}}{a_{M M F}}
$$

where $\delta=1-\left(n_{2} / n_{1}\right)^{2}$, (notice $\delta$ is twice the value of the fractional refractive index difference), in which $n_{1}$ and $n_{2}$ denote the maximum refractive index of the MMF core, and the refractive index of the MMF cladding, respectively; $a_{\mathrm{MMF}}$ denotes the radius of the GI-MMF core. Inserting Eq. 3.4 into Eq.3.5 and applying $R_{\mathrm{C}}(0) \rightarrow \infty$ for $q_{1}$, we get 


$$
\begin{gathered}
\frac{1}{q_{2}(l)}=A-i B \\
A=\frac{g\left(\lambda^{2}-\pi \omega_{0}^{4} n_{1}^{2} g^{2}\right) \sin (g l) \cos (g l)}{\lambda^{2} \sin ^{2}(g l)+\pi^{2} \omega_{0}^{4} n_{1}^{2} g^{2} \cos ^{2}(g l)} \\
B=\frac{\lambda \pi \omega_{0}^{2} n_{1} g^{2}}{\lambda^{2} \sin ^{2}(g l)+\pi^{2} \omega_{0}^{4} n_{1}^{2} g^{2} \cos ^{2}(g l)}
\end{gathered}
$$

where $\omega_{0}$ is the Gaussian beam radius of the $\mathrm{SMF} \mathrm{LP}_{01}$ mode. In seeking of the position where planar wavefront along the MMF is located, we set $A=0$ and find two sets of solutions for $l$

$$
\begin{gathered}
l_{r}=\frac{\pi}{g} N, \text { where } N=1,2,3, \ldots \\
l_{c}=\frac{\pi}{g}\left(\frac{1}{2}+N\right), \text { where } N=1,2,3, \ldots
\end{gathered}
$$

For the first set of solutions $\left(l_{\mathrm{r}}\right)$, substitution into (8.3) yields a spot radius equal to $\omega_{0}$, which is identical to that of the SMF fundamental mode. We refer such locations as "refocusing points". The second set of solutions $\left(l_{\mathrm{c}}\right)$, when inserted into (8.3) gives rise to a spot radius of

$$
\omega(l)=\frac{\lambda}{\pi \omega_{0} n_{1} g}
$$

It can be easily shown that $\omega(l) \approx\left(\mathrm{NA}_{\mathrm{SMF}} / \mathrm{NA}_{\mathrm{MMF}}\right) a_{\mathrm{MMF}}$, where $\mathrm{NA}_{\mathrm{SMF}}$ and $\mathrm{NA}_{\mathrm{MMF}}$ are the numerical apertures of SMF and MMF. For typical GI-MMF, the value of Eq.10 is approximately 2 3 times of $\omega_{0}$. We refer $l_{\mathrm{C}}$ as "collimating points", which are employed to fabricate fiber lenses.

Equations 3.9.1 and 3.9.2 imply a series of periodic focusing and defocusing when light propagates in a SMF-excited GI-MMF. For an SMS-IFPI, when the cavity length is cut to the neighborhood of $l_{\mathrm{r}}$ (3.9.1), light in the FP cavity is refocused into the lead-out SMF, leading to minimum transmission loss. On the contrary, when the FP cavity has a length near to $l_{\mathrm{c}}$ (3.9.2), 
due to the significant mode field diameter (MFD) mismatch, coupling of the cavity mode into the lead-out fiber can be very lossy.

In reality, periodic refocusing effect only exists at the beginning of the MMF section (within several centimeters), and the pattern becomes increasingly blurry along the fiber. The periodicity is dictated by the "beating" of the excited modes (as will be discussed below), and eventually all the modes become desynchronized during propagation. In order to better understand and more accurately analyze such phenomena, fiber modal analysis is necessary.

For the GI-MMF whose index profile is radially parabolic, the transverse mode profile can be well approximated by the Laguerre-Gaussian function [13]. If the MMF is excited by standard SMF, modes with azimuthal index other than zero $\left(\mathrm{LP}_{v \mu}\right.$ mode, $\left.v \neq 0\right)$ annihilate due to mode orthogonality, which greatly simplifies the following analysis. In this case the mode profile can be expressed as

$$
\psi_{\mu}(r)=A e^{-\left(r^{2} / \omega_{1}^{2}\right)} L_{\mu}\left(\frac{2 \rho^{2}}{\omega_{1}^{2}}\right)
$$

with an effective Gaussian spot size defined through

$$
\omega_{1}^{2}=\frac{2 a_{M M F}}{k_{0} n_{1} \sqrt{\delta}}
$$

and the mode number $\mu=0,1,2, \ldots$ In Eq.3.11, $\rho$ denotes radial coordinate and $L_{\mu}$ is the Laguerre polynomial of degree $\mu$. The constant $A$ is calculated by mode power normalization:

$$
\int_{0}^{\infty}\left|\psi_{\mu}(\rho)\right|^{2} \rho d \rho=1
$$

The effective refractive index for mode $\mu$ is found to be

$$
n_{e, \mu}=n_{1}\left[1-\frac{2 \sqrt{\delta}}{k_{0} n_{1} a_{M M F}} M\right]^{1 / 2}
$$


where $M=2 \mu+1$ and the propagation constant is defined by $\beta_{\mu}=k_{0} \cdot n_{\mathrm{e}, \mu}$. The mode functions in Eq.11 are orthogonal, and the $\mathrm{LP}_{01}$ mode of the SMF may couple into multiple core modes of the MMF at the first splicing plane. The field in the MMF is thus decomposed into the orthogonal modes as

$$
\psi_{M M F}(\rho)=\sum_{\mu=0}^{N-1} c_{\mu} \psi_{\mu}(\rho)
$$

$\psi_{M M F}$ is the field profile in the MMF, $N$ is the total number of modes excited, and the coupling coefficients $c_{\mu}$ are found by the overlapping integral:

$$
c_{\mu}=\int_{0}^{\infty} \psi_{S M F}(\rho) \psi_{\mu}^{*}(\rho) \rho d \rho
$$

where $\psi_{S M F}$ is the normalized field profile of the SMF $\mathrm{LP}_{01}$ mode. The field profile at any location along the MMF (assume $\mathrm{z}=0$ is the first splicing plane) is found by summing the individual orthogonal modes:

$$
\Psi(z)=\sum_{\mu=0}^{N-1} c_{\mu} \psi_{\mu}(\rho) \exp \left(-j \beta_{\mu} z\right)
$$

Numerical solutions of the field distribution in the MMF were generated using the fiber parameters listed in Table 3.2, with $\lambda=1.55 \mu \mathrm{m}$. The excitation fiber was a SMF (as listed in the table on the row "SMF"), and two types of MMF's were studied, the parameters of which were provided in the table as row "MMF1" and "MMF2". The normalized mode profiles of the five lowest MMF modes $\left(\mathrm{LP}_{00}\right.$ to $\left.\mathrm{LP}_{04}\right)$ were calculated and are depicted in Figure 3-3 for MMF1. Co-plotted is the normalized profile of the excitation SMF $\mathrm{LP}_{01}$ mode. The figure clearly shows the MFD mismatch between the SMF and MMF fundamental modes. Under this particular excitation condition, the power excitation coefficients $\left(\left|c_{\mu}\right|^{2}\right)$ for five lowest order modes of MMF1 and MMF2 were calculated based on (16) and are summarized in Table 3.3. For both fibers, the fundamental mode carries more than $80 \%$ of the total power, and less than $20 \%$ of the power is carried by higher order modes with significantly different propagation constants. The splitting of power into different modes, which propagate individually with distinct phase delays, causes interference among the modes during propagation to create particular field patterns along 
the fiber, as expressed by Eq.17. In Figure 3-4 (a), the amplitude of the field along MMF2, with $\mathrm{z}=0$ as the splicing boundary, is plotted. In Figure 3-4 (b), the field distribution for a SI-MMF, with fiber parameters identical to MMF2 is illustrated for comparison. The field distribution in the GI-MMF manifests periodic focusing along the $\mathrm{z}$ axis, whereas the field in the SI-MMF is rather complex. The reason causing this disparity lies in a special relationship among the MMF propagation constants, as discussed below.

Table 3.2. Fiber Parameters Used for Simulation.

\begin{tabular}{cccccc}
\hline \hline Fiber Type & $\begin{array}{c}\text { Core } \\
\text { Diameter } \\
(\mu \mathrm{m})\end{array}$ & $\begin{array}{c}\text { Cladding } \\
\text { Diameter } \\
(\mu \mathrm{m})\end{array}$ & $\begin{array}{c}\text { Core } \\
\text { Index } \\
(\mathrm{RIU})\end{array}$ & $\begin{array}{c}\text { Cladding } \\
\text { Index } \\
(\mathrm{RIU})\end{array}$ & $\begin{array}{c}\delta \\
(\%)\end{array}$ \\
\hline SMF & 8.4 & 125 & 1.4504 & 1.4447 & 0.78 \\
MMF1 & 50 & 125 & 1.4760 & 1.4615 & 1.96 \\
MMF2 & 62.5 & 125 & 1.4760 & 1.4500 & 3.49
\end{tabular}

Table 3.3. Power Coupling Coefficients of Five MMF Modes.

\begin{tabular}{cccccc}
\hline \hline \multirow{2}{*}{ Fiber } & 1 & 2 & 3 & 4 & 5 \\
& $\mathrm{LP}_{00}$ & $\mathrm{LP}_{01}$ & $\mathrm{LP}_{02}$ & $\mathrm{LP}_{03}$ & $\mathrm{LP}_{04}$ \\
\hline MMF1 & 0.8259 & 0.1115 & 0.0413 & 0.0132 & 0.0051 \\
MMF2 & 0.8457 & 0.1002 & 0.0371 & 0.0108 & 0.0040
\end{tabular}




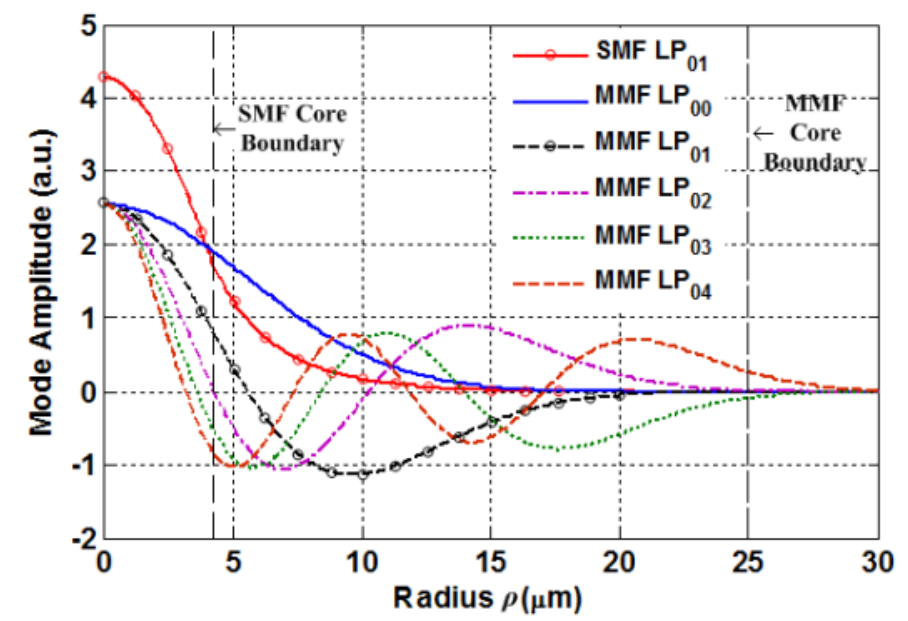

Figure 3-3. Five lowest order mode profiles of MMF1. The fundamental mode profile of the excitation SMF is also plotted for comparison. All the modes are normalized, and SMF and MMF core boundaries are marked.
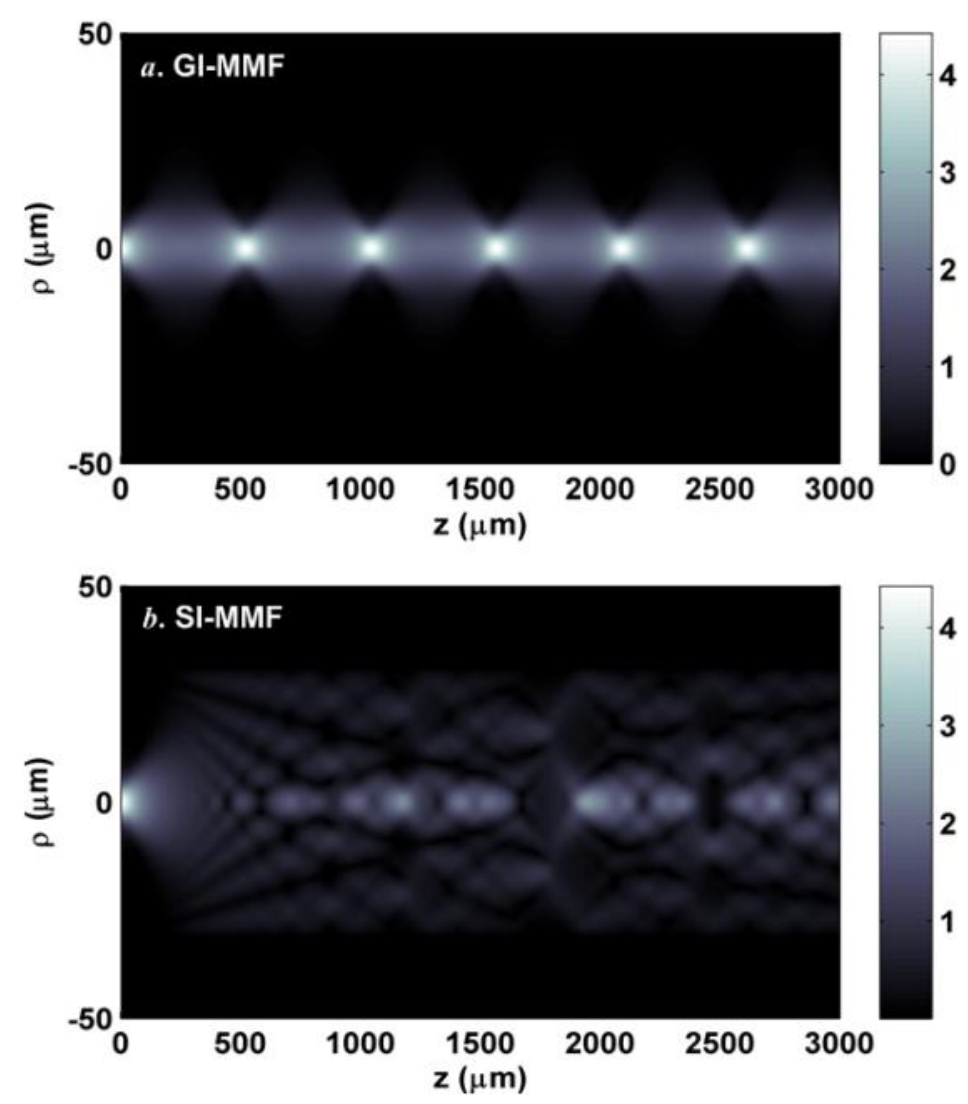

Figure 3-4. Light propagation in multimode fibers. (a) Field amplitude distribution in GI-MMF (MMF2). (b) Field amplitude distribution in SI-MMF, whose parameters are identical to MMF2. 


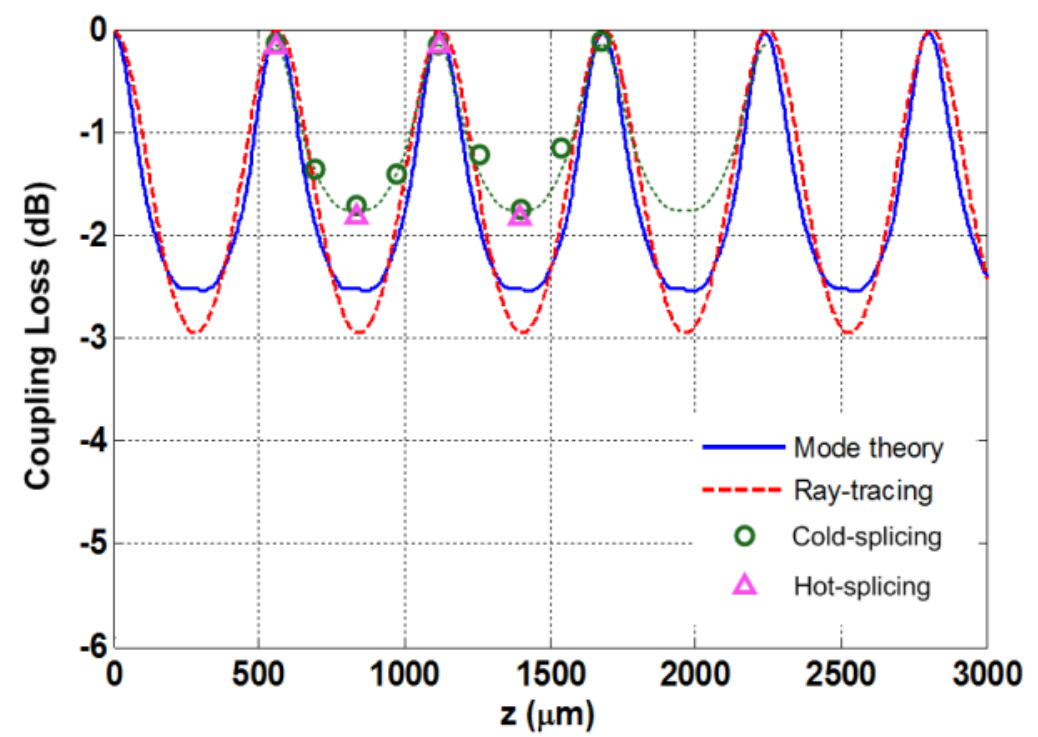

(a)

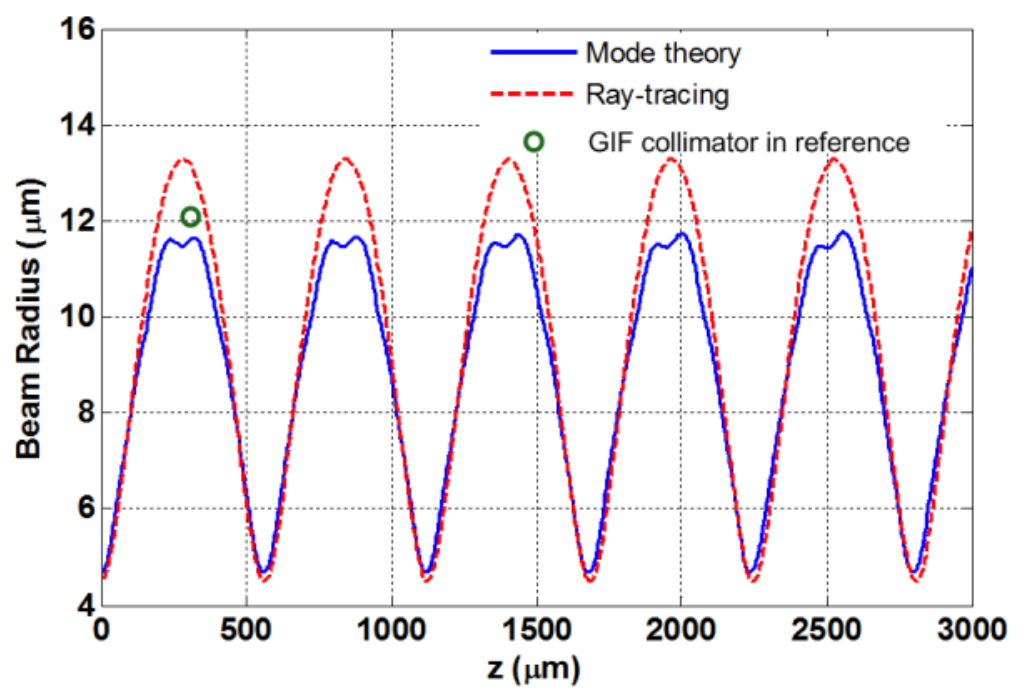

(b)

Figure 3-5. Calculated and experimental results for the coupling loss and beam radius of the GI-MMF. (a) Coupling loss variation as MMF length and (b) Beam radius variation as MMF length. For the simulation, the MMF1 fiber is selected. Corning InfiniCor600 was used for the experiment.

The periodic focusing effect in GI-MMF gives rise to periodic transmission loss. An investigation of the loss using fiber mode theory gives a clearer physical interpretation of the refocusing phenomenon. The coupling loss of the MMF can be expressed as [14]

$$
\alpha(z)=\left|\sum_{m=1}^{N} c_{m}{ }^{2} \exp \left(j \beta_{m} z\right)\right|^{2}
$$


where $m=\mu+1$. Through binomial expansion of Eq. 3.14, the difference between two neighboring values of the effective refractive index is calculated as:

$$
\Delta_{n}=n_{e, n}-n_{e, n+1}=2 \sqrt{\delta} /\left(k_{0} a_{M M F}\right)
$$

Inserting Eq. 3.19 into Eq. 3.18 leads to

$$
\alpha(z)=\left|\sum_{m=1}^{N} c_{m}{ }^{2} \exp \left[-j(m-1) k_{0} \Delta_{n} z\right]\right|^{2}
$$

With some mathematical manipulations, the loss is obtained as

$$
\alpha(z)=\sum_{p=1-N}^{N-1} \xi_{p} \exp \left(j p k_{0} \Delta_{n} z\right)
$$

in which the expansion coefficient is expressed as

$$
\xi_{p}=\sum_{n-m=p}\left(c_{n} c_{m}\right)^{2}
$$

As clearly shown in Eq. 3.21, the $\mathrm{z}$ dependent transmission loss is a Fourier series with fundamental angular frequency $k_{0} \Delta_{\mathrm{n}}$, which predicts the spatial period as

$$
\Lambda=\frac{2 \pi}{k_{0} \Delta_{n}}=\frac{\pi a_{M M F}}{\sqrt{\delta}}=\frac{\pi}{g}
$$

The periodicity shown in Eq. 3.23 is in complete agreement with the one deduced by the ray matrix method as in Eq. 3.9.1. In fact the effective index relationship in Eq. 3.19 dictates a spatial frame with which the field repeats itself according to the Fourier series Eq. 3.21; this serves as an alternative interpretation of the refocusing effect. In applying Eq. 3.19, only the first two terms in the expansion of Eq. 3.14 are retained. However, neglecting the higher order terms in Eq. 3.14 will eventually cause significant error at larger z, and the Fourier series in Eq. 3.21 will fail. Thus the refocusing can only held for a certain distance before reaching an unfocused equilibrium state, which, together with the decay of the leaky modes, sets up a steady-state propagation within centimeters [15]. 
To verify the theory, standard SMF (SMF 28e, Corning Inc.) was fusion spliced to a section of MMF (InfiniCor600, Corning, Inc.) and re-spliced back to the lead-out SMF. According to the technical specs, the MMF is similar to MMF1 in Table 3.2. The transmission loss of the device was characterized as a function of the MMF length. For the fusion splicing, we used both of the splicing conditions in Table 3.1.

For comparison, we used both the ray matrix method and the fiber modal analysis to determine the transmission loss and the beam radius of MMF1. The calculated and measured transmission loss (one-way) are co-plotted in Figure 3-5 (a), and the simulation results based on both approaches show reasonable agreement with each other and the measured data. Notice the splicing condition does not strongly affect the transmission loss, in contrast to its influence on the reflection. In Figure 3-5 (b), the calculated beam radius, using both ray matrix and modal analysis, are shown to have good agreement. We also compared the calculation result to the experimental result published in [16], in which the fiber is cut to designed length for beam collimation. The beam spot size shown in the figure in green circle was deduced from the divergence angle as reported in the paper.

The influence of the loss reduction on sensor signal demodulation was further studied with real sensors. A link with ten IFPI sensors was fabricated using standard SMF and a GI-MMF (GIF625, Thorlabs Inc.). The MMF selected has fiber parameters similar to that of MMF2 as listed in Table 3.2. Based on simulation results, the refocusing length was determined to be $517 \mu \mathrm{m}$, according to Eq. 3.9.1. Subsequently, the cavity lengths of the sensors were cut to multiples of $517 \mu \mathrm{m}$ to minimize the transmission loss. The spectrum of the link was interrogated using a swept-laser spectrometer (Si-720, Micron Optics Inc.) which offered 20,000 interrogation points from 1520 to $1570 \mathrm{~nm}$ spectral range. All the sensors were co-located inside a furnace and temperature calibration was performed. The calibration curve was fitted with a third-order polynomial, and the fitting error was characterized by its standard deviation as an estimation of the 1- $\sigma$ estimation error. Notice that the calibration was based on calculating the OPD of the sensors, and we used the "direct OPD" approach, as elaborated in [17], Eq. 3.21, for the signal demodulation. 


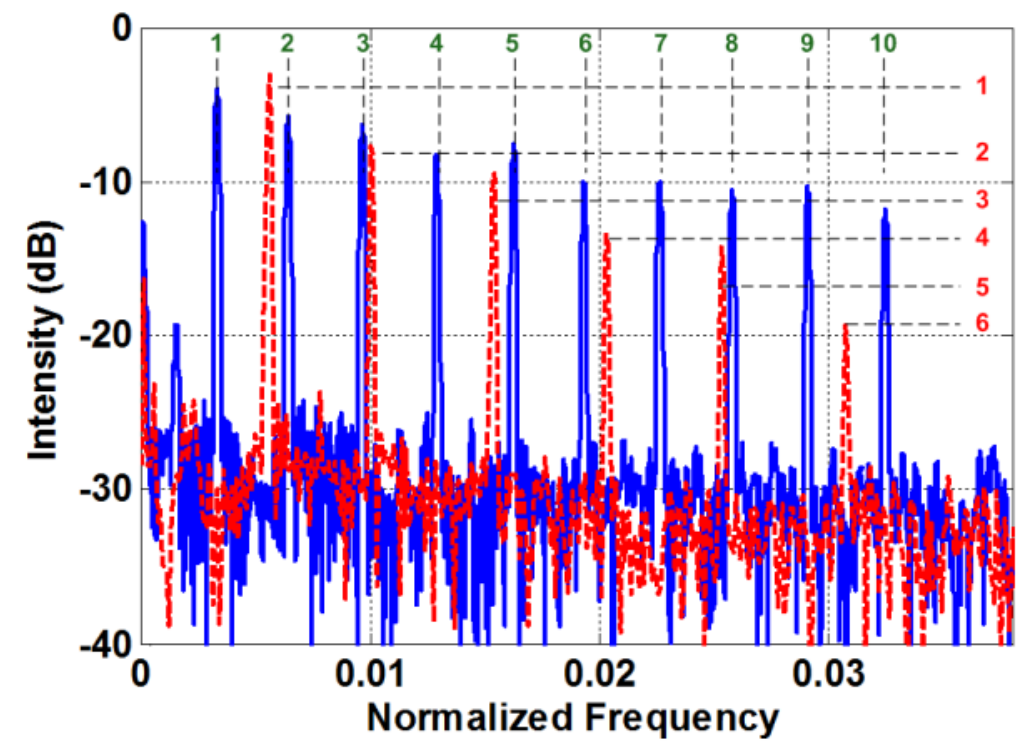

(a)

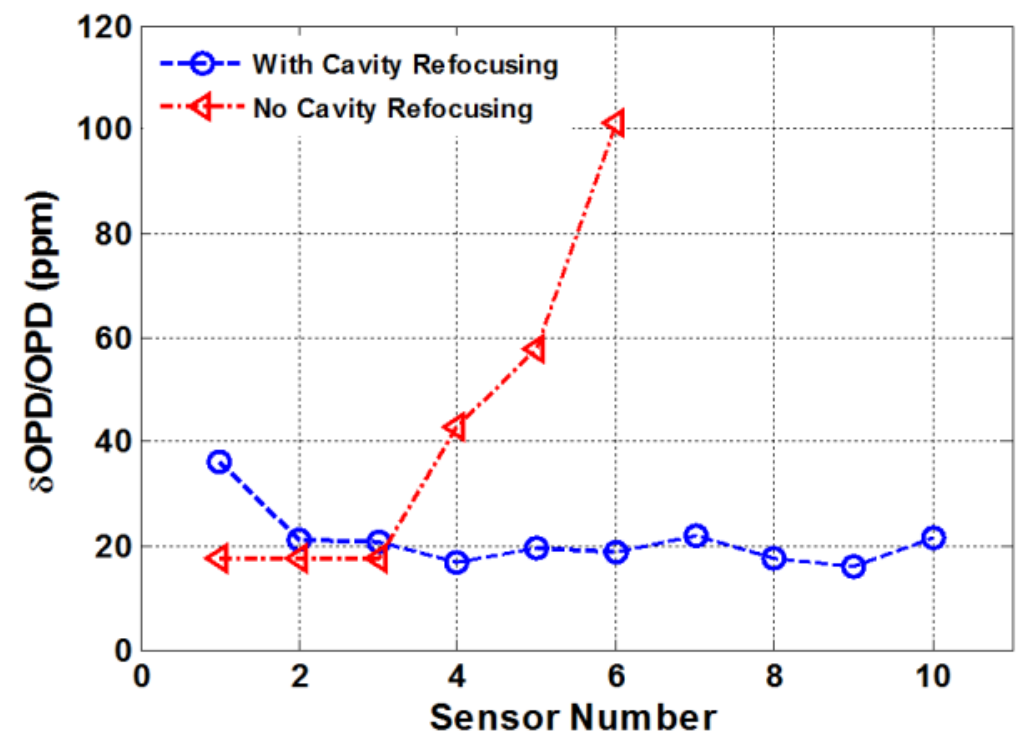

(b)

Figure 3-6. Experimental results showing the benefit of IFPI cavity length control. In (a), the FFT of the spectra for a link with (solid) and without (dashed) refocusing are compared. The relative OPD estimation error for each sensor in the links is plotted in (b).

For comparison, another link with six sensors was fabricated and tested. Instead of having controlled length to minimize the transmission loss, the sensors were cut into lengths in rough multiples of $1000 \mu \mathrm{m}$ (from 1000 to $6000 \mathrm{um}$ ). The link had significantly increased transmission loss (roundtrip loss averaged nearly $3 \mathrm{~dB}$ per sensor), in vivid contrast to the first link whose 
average loss was $<0.5 \mathrm{~dB}$ per sensor. This is clearly shown in the Fourier transform of the sensor spectra in Figure 3-6 (a), where the harmonic peaks due to each individual sensor are visible.

In Figure 3-6 (b), the relative OPD estimation error (normalized to OPD) for the sensors in both links is plotted. While the sensors in link 1 demonstrate no quality reduction toward the end of the link, the last three sensors in the non-optimized link show dramatically increased inaccuracy as a result of reduced SNR. A natural benefit from the loss reduction is an increase in multiplexing capacity.

\subsection{Sensor signal processing}

\subsubsection{Modeling of the IFPI structure and the introduction of the additional phase}

\section{a. Exact field expression}

Light in the SMF is carried by $L P_{01}$ mode of SMF with normalized mode profile $\Phi_{0}$. At the interface $R_{1}$, the mode energy is split into several parts carried separately by $L P_{0 k}$ modes with normalized mode profile $\Phi_{k}$ in the MMF (here we made the assumption that the MMF is weakly guided, step-index fiber for simplicity of analysis). Modes in the MMF with azimuthal number other than zero will not be exited due to mode orthogonality. For each mode, the coupling coefficient is defined as $\eta_{0 \rightarrow k}=\iint_{R_{1}} \phi_{0} \phi_{k}^{*} d x d y$. Being reflected by $R_{2}$ and propagating to $R_{1}$, the $k$ th mode profile can be expressed as $r \eta_{0 \rightarrow k} \phi_{k} \exp \left[j\left((2 \pi / \lambda) O P D_{k}+\pi\right)\right]$, where $O P D_{k}=2 n_{k} L, n_{k}$ is the effective index of the $k$ th mode. At interface $R_{l}$, all these modes will be re-coupled into the fundamental $L P_{01}$ mode of the single mode fiber with coupling coefficient $\eta_{k \rightarrow 0}=\iint_{R_{1}} \phi_{k} \phi_{0}^{*} d x d y=\eta_{0 \rightarrow k}^{*}$. As a result, the contribution to the total mode profile by each mode can be described as $r\left|\eta_{0 \rightarrow k}\right|^{2} \phi_{0} \exp \left[j\left(\frac{2 \pi}{\lambda} O P D_{k}+\pi\right)\right]$, and the total field profile at $R_{l}$ can be expressed as:

$$
\phi_{s}=r \phi_{0}\left(1+\sum_{k=1}^{N} \eta_{k}^{2} \exp \left[j\left(\frac{2 \pi}{\lambda} O P D_{k}+\pi\right)\right]\right)
$$


where $\eta_{k}=\left|\eta_{0 \rightarrow k}\right|$ is a real number and $N$ modes are assumed to be excited in the MMF. Based on the expression for the total field above, we can find the reflection intensity from the sensor as:

$$
I=\iint_{R_{1}} \phi_{s} \phi_{s}^{*} d x d y=r^{2}|1+\Sigma|^{2}
$$

where $\Sigma=\sum_{k=1}^{N} \eta_{k}^{2} \exp \left[j\left(\frac{2 \pi}{\lambda} O P D_{k}+\pi\right)\right]$ is an effective vector to be discussed in more details in the following section. Also we have already applied the normalization condition $\iint_{R_{1}} \phi_{0} \phi_{0}^{*} d x d y=1$.

\section{b. Two-mode excitation}

The expression for the total field intensity deduced from the last section (Eq.3.25) is comprehensive but relatively complicated. In this section we consider a much simpler case in which only two modes are excited in the MMF with similar effective refractive indices; this analysis can provide us with illustrative understanding of how intermodal dispersion affects the sensor spectrum. Assume that the effective indices of the two modes to be $n_{1}$ and $n_{2}$, respectively, and accordingly the $O P D$ experienced by the two modes to be $O P D_{1}=2 n_{1} L$ and $O P D_{2}=2 n_{2} L$, define $\triangle O P D=O P D_{1}-O P D_{2}$ such that $O P D_{2}$ can be expressed by $O P D_{2}=O P D_{1}-\triangle O P D$. The total reflected spectrum can be expressed as

$$
\begin{aligned}
& I= \\
& 1+\eta_{1}^{4}+\eta_{2}^{4}+2 \eta_{1}^{2} \cos \left(O P D_{1} \cdot k_{0}\right)+2 \eta_{2}^{2} \cos \left(\left(O P D_{1}-\Delta O P D\right) k_{0}\right)+2 \eta_{1}^{2} \eta_{2}^{2} \cos \left(\Delta O P D \cdot k_{0}\right)
\end{aligned}
$$

In the above equation, the fourth and fifth terms on the right hand side describe the interference of the modes with themselves, and the last term accounts for the interference between the two modes. After some mathematical manipulations, Eq.3.26 can be rewritten as:

$$
I=r^{2}\left\{1+\Gamma+2 \sqrt{\Gamma} \sin \left[O P D_{1} k_{0}+\varphi\left(\Delta O P D, k_{0}\right)+\pi\right]\right\}
$$

where

$$
\Gamma=\eta_{1}^{4}+\eta_{2}^{4}+2 \eta_{1}^{2} \eta_{2}^{2} \cos \left(\Delta O P D \cdot k_{0}\right)
$$




$$
\varphi\left(\Delta O P D, k_{0}\right)=\left\{\begin{array}{c}
\arctan \left(\frac{\eta_{1}^{2}+\eta_{2}^{2} \cos \left(\Delta O P D \cdot k_{0}\right)}{\eta_{2}^{2} \sin \left(\Delta O P D \cdot k_{0}\right)}\right), \sin \left(\triangle O P D \cdot k_{0}\right) \geq 0 \\
\pi-\arctan \left(\frac{\eta_{1}^{2}+\eta_{2}^{2} \cos \left(\Delta O P D \cdot k_{0}\right)}{\left|\eta_{2}^{2} \sin \left(\Delta O P D \cdot k_{0}\right)\right|}\right), \sin \left(\Delta O P D \cdot k_{0}\right)<0
\end{array}\right.
$$

It is not difficult to find that at the limit $\eta_{2}^{2}<<\eta_{1}^{2}$ (which means the second mode is excited so weakly that almost all the power is carried by the first mode), Eq.3.27 reduces to the case when the additional phase term equals to zero. As the second mode emerges, the amplitude and phase of the sinusoid will be modulated. The phase term $\varphi\left(\triangle O P D, k_{0}\right)$ as a function of $\triangle O P D \cdot k_{0}$ has been plotted in Figure 3-7. To plot the figure, the wavelength span was set from 1400 to 1700 $\mathrm{nm}$, the effective refractive indices of mode 1 and mode 2 were set to be 1.448 and 1.441 , respectively, the cavity length was set to $1 \mathrm{~mm}$, and four different cases with excitation ratio ( $\left.\eta_{1}^{2} / \eta_{2}^{2}\right)$ of $6: 4,7: 3,8: 2$ and 9:1 were compared.

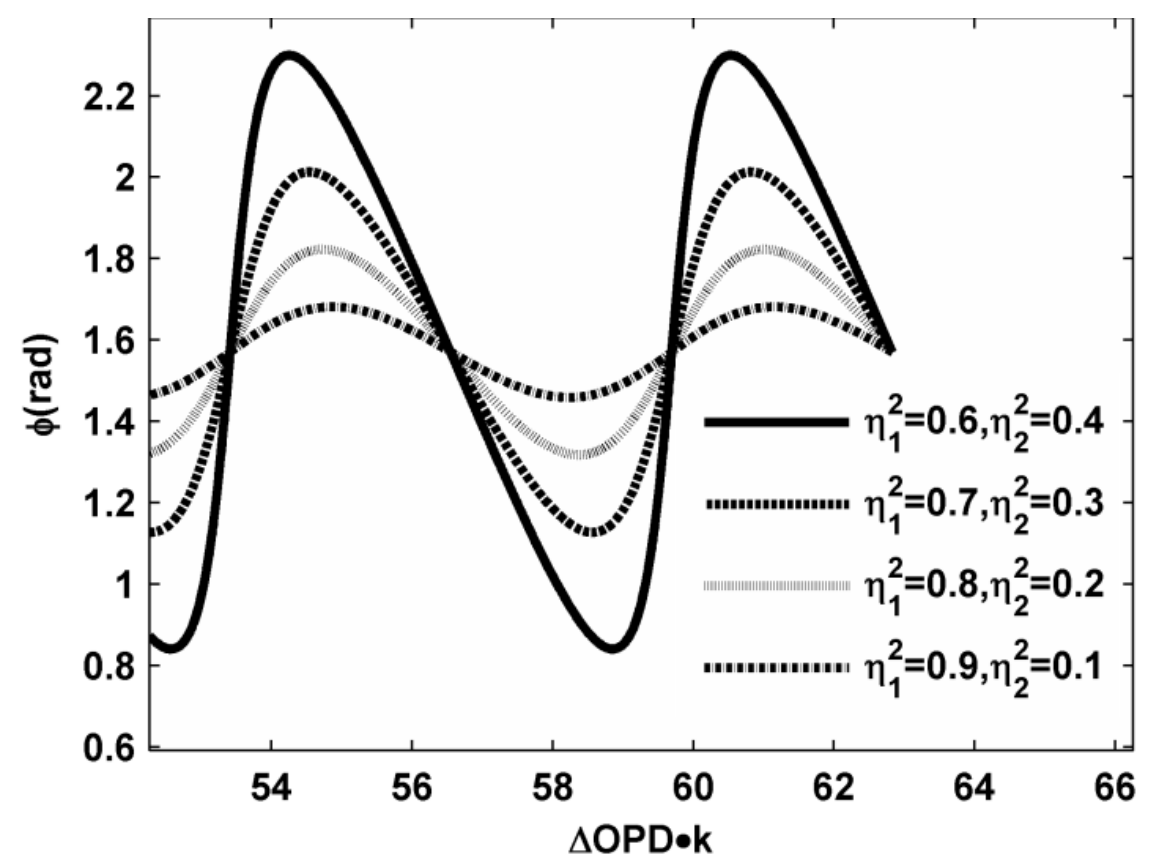

Figure 3-7. Additional phase term for a two-mode cavity at different excitation ratios.

\section{c. Rotating vector picture}

In the previous section we only considered the excitation of two modes inside the MMF cavity. In reality, the number of modes excited can be much more, which complicates the problem. 
From Eq.3.25, the received intensity is proportional to $|1+\Sigma|^{2}$ where the vector $\Sigma$ is defined as $\Sigma=\sum_{k=1}^{N} \eta_{k}^{2} \exp \left[j\left(\frac{2 \pi}{\lambda} O P D_{k}+\pi\right)\right]$. The received power can be expressed as:

$$
I \sim|1+\Sigma|^{2}=1+|\Sigma|^{2}+2|\Sigma| \cos (\psi), \text { where } \psi=\angle \Sigma
$$

Eq. 3.28 leads to the conclusion that the total phase in the interference spectrogram can be directly obtained from the angle of the vector $\Sigma$. In the following discussion, for simplicity without losing generality, we omit the phase term $\pi$ in the expression of vector $\Sigma$ :

$$
\Sigma=\sum_{k=1}^{N} \eta_{k}^{2} \exp \left[j\left(\frac{2 \pi}{\lambda} O P D_{k}\right)\right]
$$

where $O P D_{k}=2 n_{k} L, n_{k}$ is the effective refractive index of the $k$ th mode. It is straightforward to find out that $\Sigma$ is the sum of $N$ vectors representing $N$ orthogonal modes (define mode vector $\left.v_{k}=\eta_{k}^{2} \exp \left[j\left((2 \pi / \lambda) O P D_{k}\right)\right]\right)$, the modulus of each vector is the square of the magnitude of the mode coupling coefficient between the given mode and the $L P_{01}$ mode of the SMF; the phase of each vector is simply the product of the vacuum wavenumber and the effective $O P D$ of that mode. Figure 3-8 illustrates how the total field vector is related to each individual mode vector. Totally five modes were assumed to be excited.

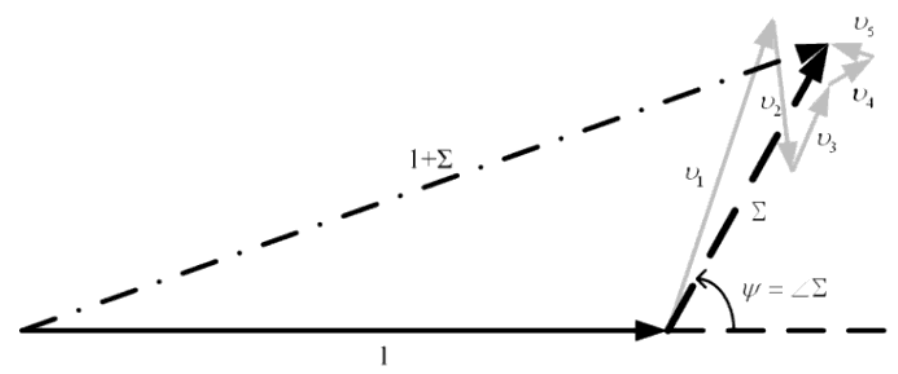

Figure 3-8. Relationshio between the total field vector and five individual mode vectors. 
In the interference spectrogram, as the wavenumber $k$ increases, all the $N$ individual mode vectors $\left(v_{k}\right)$ will rotate at different angular speed $O P D_{k}=2 n_{k} L$, the mode vector of the fundamental $L P_{01}$ mode rotates at the fastest speed (corresponding to the largest effective refractive index), while the mode vector of $L P_{O N}$ mode rotates the slowest; the differences among rotational speeds of all the mode vectors are very small because $n_{k}$ 's have very similar values. Although the individual vectors rotate at similar speeds, their pointing directions are almost arbitrary because the wavenumber $k$ and the cavity length $L$ are both very large numbers. The effective vector $\Sigma$ rotates at a speed that depends simultaneously on the rotational speed of each vector and their relative orientations. If most of the mode vectors align at similar directions, the rotating speed of the effective vector can be slower than any of the individual vectors; there are also occasions where its rotational speed is faster than any of the individual vectors (see Figure 3-9 for an example).

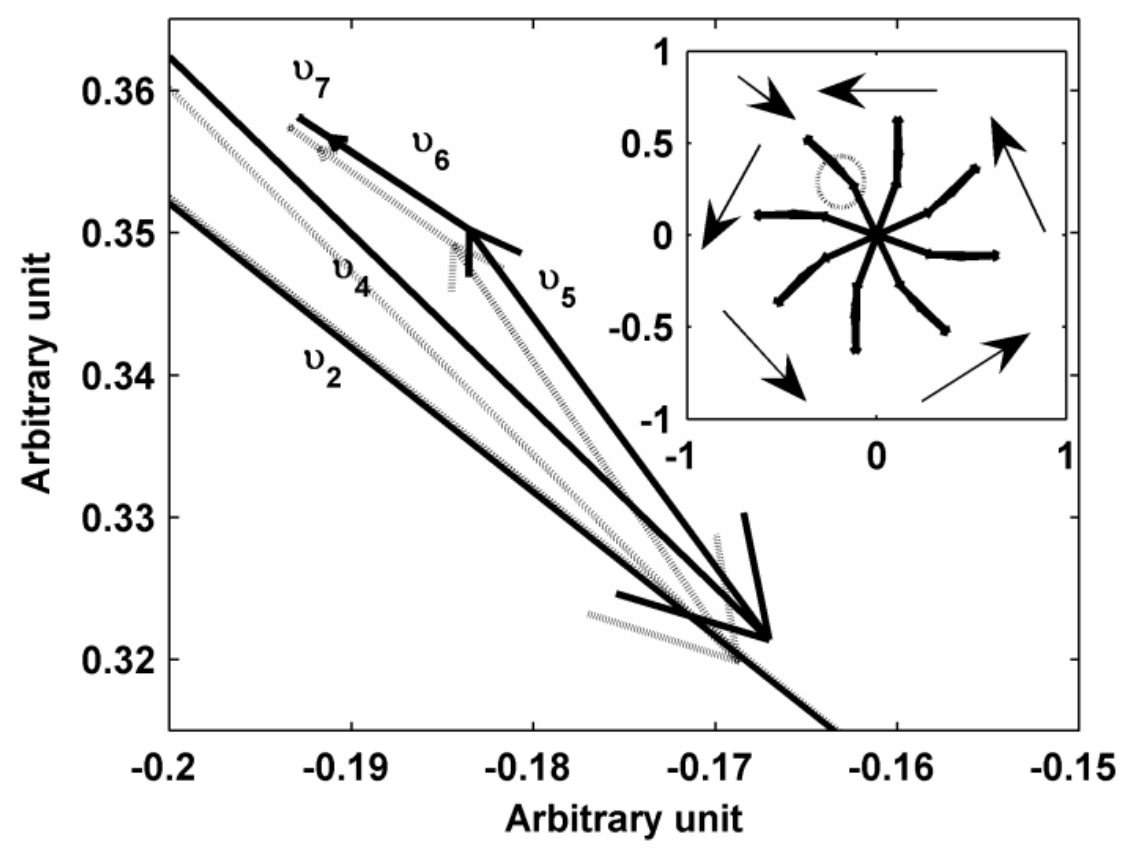

Figure 3-9. Zoom-in view of the rotating vectors. Nine groups of vectors are plotted with equal angular spacing such that $v_{1}$ rotates exactly $2 \pi$. Dark lines: first group of vectors (beginning position); gray lines: last group of vectors (end position: $v_{1}$ coincides with $v_{1}$ in the first group, $v_{2}$ is a little behind $v_{2}$ in the first group; the effective vector $\Sigma$ is ahead of $\Sigma$ in the first group). Inset: global view of the vector rotation. Upper-left arrow: position of the first and last groups of vectors; other arrows illustrate the rotational direction of the vectors. 
Figure 3-9 plots the mode vectors of all the seven excited modes in a step-index MMF with $n_{\text {core }}=1.448, n_{\text {clad }}=1.434$, and $r_{\text {core }}=25 u m$, excited by SMF with $n_{\text {core }}=1.445, n_{\text {clad }}=1.440$, and $r_{\text {core }}=4.2 \mathrm{um}$. In the simulation, the cavity length is $2 \mathrm{~mm}$, and the wavelength is set to be $\lambda=1550 \mathrm{~nm}$. Increasing wavenumber $k$ results in rotation of mode vectors, while the effective vector rotates correspondingly. Totally nine groups of vectors are plotted with equal angular spacing, such that the fundamental mode vector $\left(v_{1}\right)$ of the ninth group coincides with that of the first group, which means by increasing $k, v_{1}$ rotates exactly $2 \pi$. Since the fundamental mode travels the fastest, all the higher order modes rotate at slower rates; none of the higher order modes rotates as far as $2 \pi$. However, the effective vector rotates faster than the fundamental mode vector, as shown in Figure 3-9. The end of the cascaded gray arrows (which represents the effective vector after $v_{1}$ rotates by $2 \pi$ ) falls ahead of the end of the cascaded dark arrows (which represents the effective vector before rotation), indicating a faster rotational speed of the effective vector than the fundamental mode vector $v_{l}$.

As $k$ increases, all the vectors begin to rotate; while each individual mode vector rotates at constant speed, the effective vector does not, even though the speed variation is not significant if

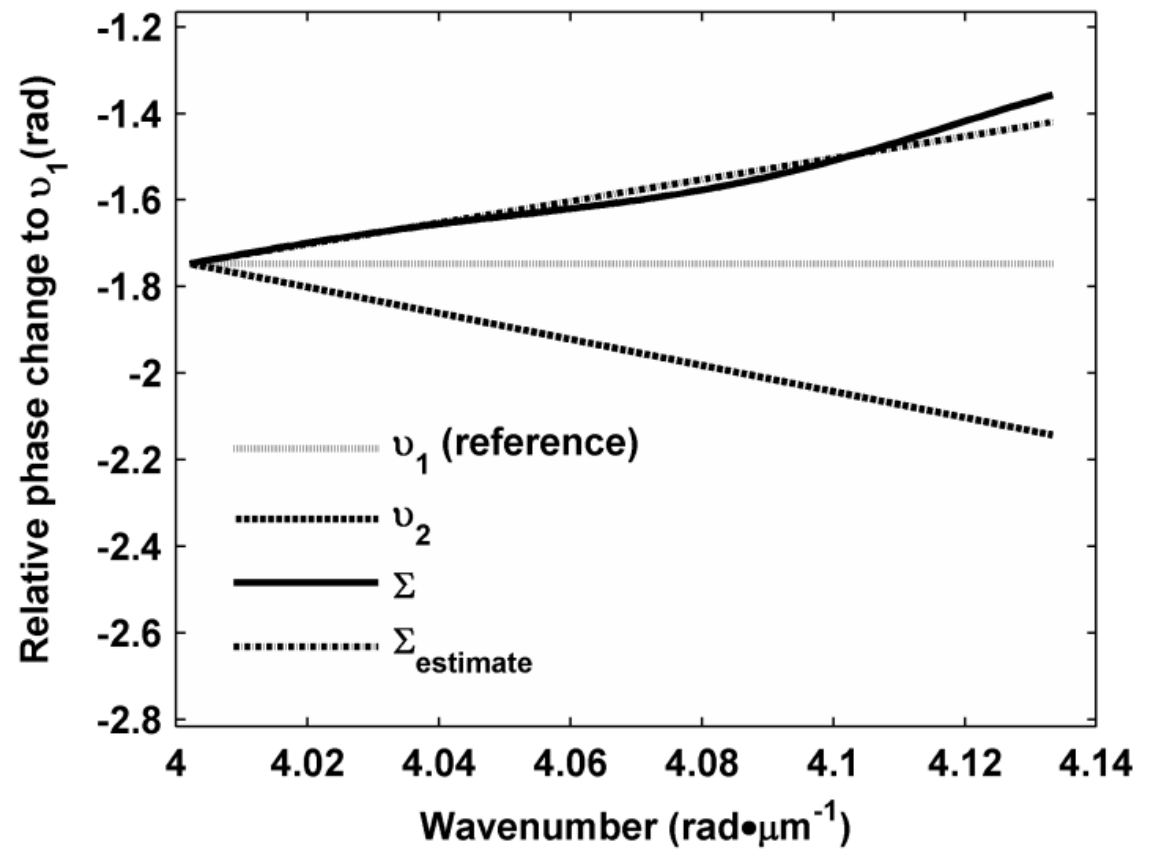

Figure 3-10. Relative phase shift to $L P_{01}$ mode as $k$ increases. Plotted are phase shifts of $L P_{02}$ mode, the effective vector $\Sigma$, and a virtual mode with $n_{\text {eff }}=n_{\text {estimate }}$. 
$k$ scans in a narrow range (within the spectral range of the interference spectrogram). Taking the rotational speed of $v_{1}$ as reference, the relative speed for any other vector can be obtained by subtracting the speed of $v_{1}$ from its own speed. At any given wavenumber $k$, this relative speed is proportional to the phase difference between that vector and the $L P_{01}$ vector. For the cavity we examined to plot Figure 3-9, when the wavelength is scanned from 1520-1570nm, the relative phase shift (taking $L P_{01}$ vector as the reference) of the following vectors are plotted in Figure 5: $L P_{02}$ (at a lower rotating speed), the effective vector $\Sigma$ (at a faster, varying speed), and the linear fitting of the rotating speed of $\Sigma$ (represents a virtual mode vector that best approximates the rotating speed of the effective vector).

\section{d. The spectrum of GI-MMF based IFPI}

The mode theory developed in Section 3.2 is extended in this subsection to treat the IFPI spectrum, in which the analysis in Section 3.2 is employed to yield the explicit expression for the MMF fields. This is of great importance to any white-light interferometry (WLI) [18] based sensor signal processing method.

The spectrum of the SMS-IFPI, normalized with respect to the input power, is expressed as [19]

$$
I=R\left|1-\sum_{m=1}^{N} c_{m}{ }^{2} \exp \left(-j 2 \beta_{m} L\right)\right|^{2}
$$

where $R$ is the reflectivity of the splicing point and L is the length of the FP cavity. For WLI based signal demodulation, the optical path difference (OPD), defined as

$$
O P D=2 \bar{n} L
$$

is found by estimating the frequency of the spectrogram [21], in which $\bar{n}$ is the estimated index. By removing the phase term associated with $\bar{n}$ out of the superposition in Eq. 3.30 and rearranging the rest terms, applying Eq. 3.19 for GI-MMF, the spectrum is subsequently found as

$$
I=R\left[1+|\xi|^{2}+2|\xi| \cos \left(2 \bar{n} k_{0} L+\theta+\pi\right)\right]
$$


where the complex variable $\xi$ is defined by

$$
\xi=|\xi| e^{j \theta}=\exp \left[j 2 \delta_{n} k_{0} L\right] \cdot \sum_{m=1}^{N} c_{m}{ }^{2} \exp \left[-j 2(m-1) k_{0} \Delta_{n} L\right]
$$

with $\delta_{\mathrm{n}}=n_{\mathrm{e}, 1}-\bar{n}$ being the difference between the estimated index and the highest effective index of the MMF. The absolute value of $\xi$ affects the fringe visibility of the spectrum as

$$
F V(L)=\frac{2|\xi|}{1+|\xi|^{2}}
$$

The additional phase term $\theta$, which plays very important role in WLI signal processing [17], is defined by the phase of $\xi$. This additional phase, according to Eq. 3.33, is composed of two terms, a linear term (with respect to L) associated with $\delta_{\mathrm{n}}$ and an oscillatory one associated with the superposition term. Our numerical analysis for both GI-MMF and SI-MMF yields several conclusions regarding the phase terms in Eq. 3.32 and Eq. 3.33. Because $c_{1}$ is typically significantly larger than the rest of the coupling coefficients, the oscillatory phase is trivial compared with the linear phase in Eq. 3.33. For GI-MMF, the index difference $\delta_{\mathrm{n}}$ is fairly stable with $L$, in contrast to the case of SI-MMF in which $\delta_{\mathrm{n}}$ changes drastically. As a result, for GIMMF, the additional phase term $\theta$ changes nearly linearly with $L$ (slope defined by the linear phase term in Eq. 3.33 with little ripples superimposed), whereas for SI-MMF the additional phase term would change rapidly and nearly randomly. Such phenomena are illustrated in Figure 3-11, where the additional phase term in the spectrum of a SMS-IFPI employing MMF2, as a function of cavity length is plotted. The phase term of an IFPI employing a SI-MMF, with all the fiber parameters identical to MMF2 is also plotted for comparison. In plotting these curves, for both GI-MMF and SI-MMF, one thousand spectrums were created based on Eq. 3.30, with $L$ evenly distributed from 500 to $3500 \mu \mathrm{m}$ and all the other fiber parameters fixed. Then, the OPD estimation algorithm in [17] was applied for both groups of data to calculate the additional phase terms, and the results were plotted as a function of cavity length. One should note that the rapid change in Figure 3-11(b) is attributed to the change of $\delta_{\mathrm{n}}$ with $L$, not the oscillatory phase of the superposition term in Eq. 3.33. 


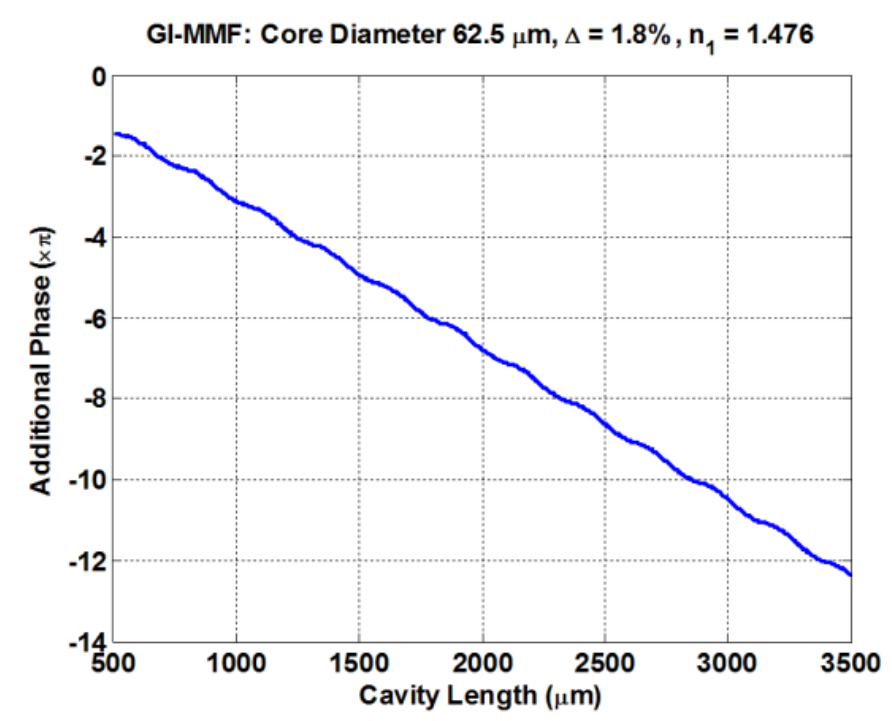

(a)

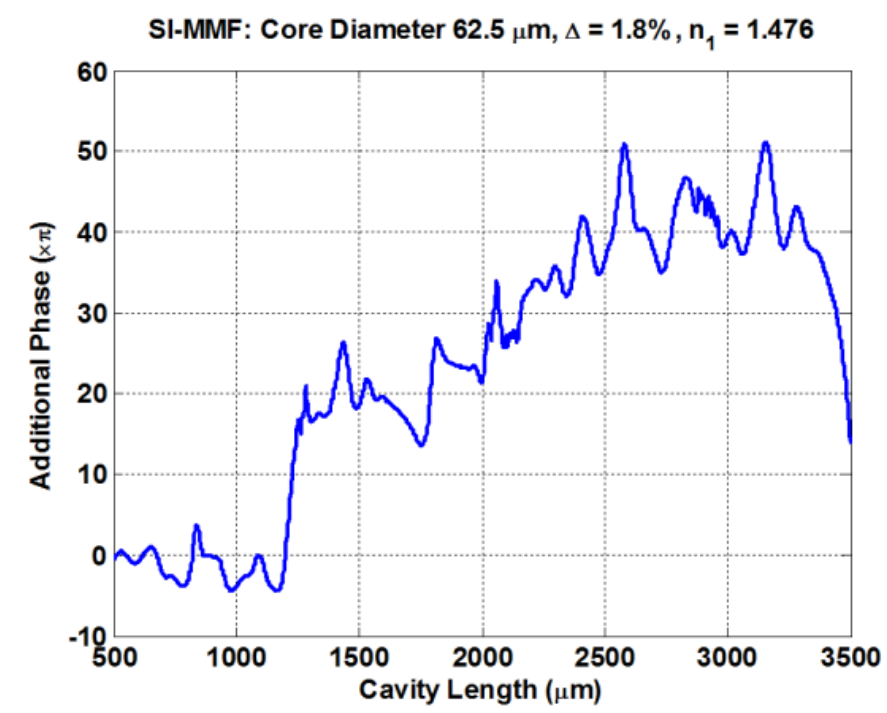

(b)

Figure 3-11. The evolution of the additional phase term $\theta$ with cavity length $L$. (a) The additional phase term in GI-MMF (MMF2). (b) The additional phase term in SI-MMF, whose parameters are identical to MMF2.

Equation 3.33 indicates that the additional phase term in the SMS-IFPI sensor spectrum Eq. 3.32 is composed of two parts. The linear part $2 \delta_{\mathrm{n}} k_{0} L$ is generated by the difference between the refractive index of the MMF fundamental mode $\left(n_{\mathrm{e}, 1}\right)$ and the estimated refractive index $(\bar{n})$, denoted as $\delta_{\mathrm{n}}$. For GI-MMF, $\delta_{\mathrm{n}}$ is stable, and the contribution by $2 \delta_{\mathrm{n}} k_{0} L$ is almost linear with $L$. The other component in the additional phase is 


$$
\theta_{\text {osc }}(L)=\angle\left\{\sum_{m=1}^{N} c_{m}{ }^{2} \exp \left[-j 2(m-1) k_{0} \Delta_{n} L\right]\right\}
$$

which oscillates around zero with small amplitude due to the dominant coupling coefficient $c_{1}$ in the superposition. As such, replacing $\theta$ with $2 \delta_{\mathrm{n}} k_{0} L$ provides reasonably good approximation for the additional phase. Furthermore, the linear term can be written as $\left(\delta_{\mathrm{n}} / \bar{n}\right) k_{0} O P D$, upon which we take the derivative with respect to $O P D$, and get

$$
\frac{d \theta}{d O P D} \approx \frac{\delta_{n}}{\bar{n}} k_{0}
$$

In real measurement, $O P D$ can be estimated with very high accuracy as $O P D_{\mathrm{e}}$, and $\theta$ can also be estimated by the algorithm. Thus the value of $\mathrm{d} \theta / \mathrm{d} O P D$ is readily obtained after the sensor is calibrated. Because $L$ can be controlled during sensor fabrication with accuracy of $\pm 5 \mu \mathrm{m}$, the value of $\bar{n}$ is estimated by $O P D_{\mathrm{e}} / 2 L$. From Eq. 3.36 the value of $\delta_{\mathrm{n}}$ is obtained as $\delta_{\mathrm{n}}=\bar{n}($ $\mathrm{d} \theta / \mathrm{d} O P D) / k_{0}$.

On the other hand, from the numerical model described in Section 3.2, the calculation of the

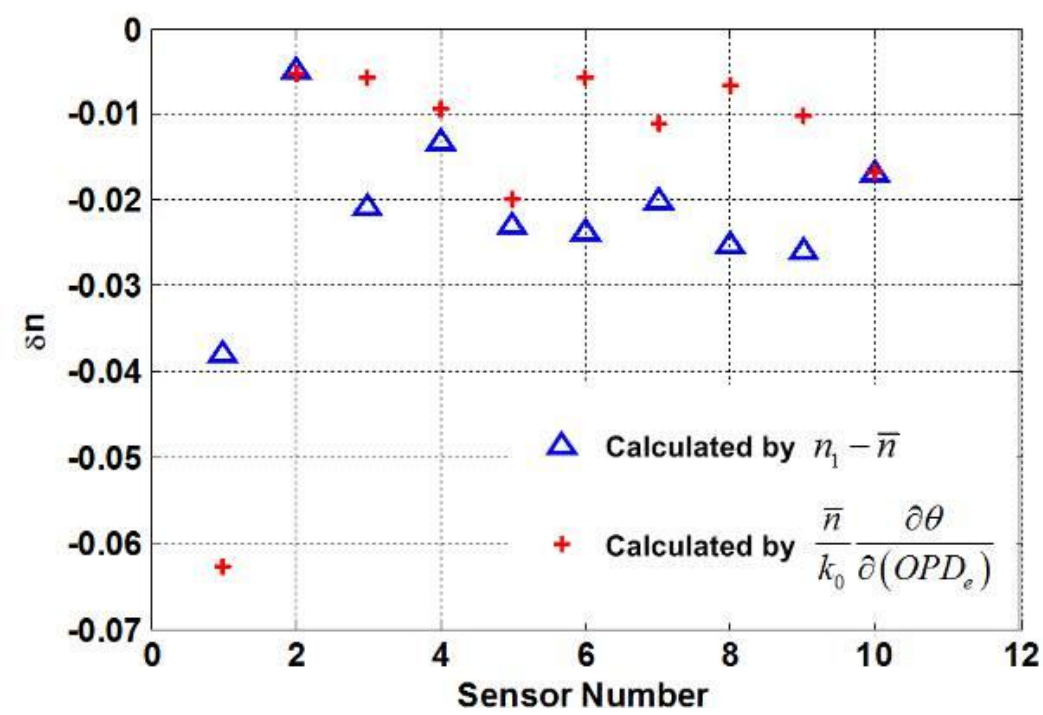

Figure 3-12. Verification of the analysis of the additional phase term $\theta$ in (26). The experimental demonstration is achieved by comparing the estimation of $\delta$ n by two approaches for ten sensors multiplexed in a link.

effective indices of the excited modes of MMF2 provides estimation of $n_{\mathrm{e}, 1}$, and an alternative 
estimation of $\delta_{\mathrm{n}}$ is readily obtained by its definition $\delta_{\mathrm{n}}=n_{\mathrm{e}, 1^{-}} \bar{n}$. In Figure 3-12, the $\delta_{\mathrm{n}}$ values of the IFPI sensors in the link whose Fourier spectrum is depicted as the solid curve in Figure 3-11(a) are calculated using the above two methods and co-plotted. The figure shows acceptable accuracy, and the discrepancies are attributed to the uncertainties associated with the estimation of $\theta, O P D, \bar{n}$ and $n_{\mathrm{e}, 1}$.

According to Eq. 3.36, the additional phase is proportional to OPD and $\delta_{\mathrm{n}}$. A non-constant additional phase will potentially lead to abrupt discontinuities in the demodulated signal, as analyzed in [17]. As a result, minimizing the value of $\mathrm{d} \theta / \mathrm{d} O P D$ is essential for reliable sensor performance. As predicted by Eq. 3.36 and demonstrated in Figure 3-12, the longer the cavity length, the larger the total additional phase change. Minimization of the additional phase change is also achievable via reducing the value of $\delta_{\mathrm{n}}$, which is not a trivial task. The value of $\delta_{\mathrm{n}}$ (e.g., the difference between the "apparent" effective index and the index of the MMF fundamental mode) is directly associated with the modal excitation condition in the MMF. For real cases, the modal excitation can be more complex than modeled in Section 3.2, as a result the additional phase is not as linear as in Figure 3-11(a).Practical values of $\delta_{\mathrm{n}}$ can be large (as shown in Figure 3-12), which leads to a much larger rate of phase change than predicted in Figure 3-11(a).

\subsubsection{Sensor signal processing and the total phase approach}

\section{a. Non-constant phase-induced OPD demodulation jumps}

To interrogate a low-finesse FP sensor, the phase of the periodic fringe pattern is measured at either a fraction or all of the sampling points in the spectrogram [20, 21, 22, 23]. This interference spectrum is represented by $S(k) \propto \cos [\Phi(k)]$, where $k=2 \pi / \lambda$ is the optical wavenumber, $\lambda$ is the wavelength, $\Phi(k)$ is the total interferogram phase which is expressed as $\Phi(k)=k \cdot \mathrm{OPD}+\varphi_{0}$, where $\varphi_{0}$ is an additional phase term caused by beam reflection and propagation $[19,24]$. In traditional OPD-based demodulation, the additional phase term $\varphi_{0}$ is usually assumed to be constant during measurement process and needs to be pre-calibrated [21, 22]. However, physical changes in IFPI sensors can cause this term to vary during measurement (as discussed in Section 3.3.1, and in Ref [19, 24]). 


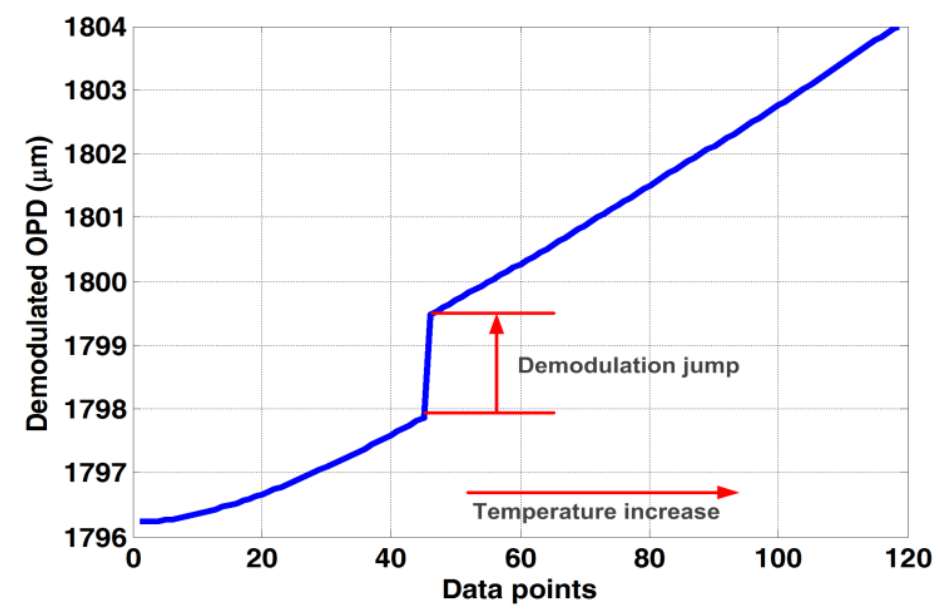

Figure 3-13. Output of a high-quality SMS-IFPI sensor under smoothly increasing temperature.

When interrogated using traditional OPD techniques, the sensor experiences an abrupt jump in its demodulated signal.

The SMS-IFPI sensors were typically interrogated using a swept-laser interrogation system (Micron Optics Si-720) over a spectral range of 1520-1570nm with spectral resolution of $2.5 \mathrm{pm}$, dynamic range of $70 \mathrm{~dB}$, and a scanning rate of $0.5 \mathrm{~Hz}$. Each collected spectrum was subsequently processed using a peak tracking method, in which local curve fitting is used to identify the exact locations of every fringe peak in the interferogram, and their corresponding fringe orders are determined to measure OPD [20, 21, 23]. The additional phase term $\varphi_{0}$ is assumed to be constant in this algorithm [21].

Several sensors were fabricated and tested under continuously-increasing temperature in a tube furnace. Figure 3-13 clearly shows the OPD of a high-quality sensor beginning to lengthen as the temperature increases. However, even with a SNR of greater than $30 \mathrm{~dB}$, the sensor experiences an abrupt jump in its measured OPD. From these results, it is clear that noise is not the sole cause of the OPD discontinuity problem. The following section provides detailed analysis to show that a time-varying initial phase $\varphi_{0}$ is the source of the observed OPD jumps.

In order to better expose the influence of the phase shift on the demodulation process, the analysis begins with recording a reference spectrum and tracking the spectral changes relative to it. At a reference measurement condition (e.g., room temperature), the following equation holds: 


$$
O P D^{(0)} \cdot\left[k_{m}^{(0)}\right]_{N \times 1}+\varphi_{0} \cdot[I]_{N \times 1}=2 \pi \cdot[m]_{N \times 1}
$$

where $\left[k_{\mathrm{m}}{ }^{(0)}\right]$ denotes a vector containing the $N$ wave-numbers corresponding to $N$ peak positions in the interference spectrum, $[m]$ denotes their corresponding fringe-orders, and $[I]=[1,1, \ldots 1]^{\mathrm{T}}$. At another measurement condition, both the OPD and the phase in the spectrum are modified by the change of the measurand, and accordingly Eq. 3.37 evolves into the following equation:

$$
O P D^{(1)} \cdot\left[k_{m}^{(1)}\right]_{N \times 1}+\varphi_{0} \cdot[I]_{N \times 1}+\Delta \varphi_{0} \cdot[I]_{N \times 1}=2 \pi \cdot[m]_{N \times 1}+2 \pi n^{(1)} \cdot[I]_{N \times 1}
$$

where $\Delta \varphi_{0}$ accounts for the additional phase change and $n^{(1)}$ is an integer denoting the fringeorder shift. By subtracting Eq.3.37 from Eq. 3.38, the following equation for estimating the modified OPD is obtained:

$$
\left[O P D_{m}^{(1)}\right]_{N \times 1}=\left[k_{m}^{(0)} / k_{m}^{(1)}\right]_{N \times 1} \cdot O P D^{(0)}+\psi \cdot\left[1 / k_{m}^{(1)}\right]_{N \times 1}
$$

where $\psi=2 \pi n^{(1)}-\Delta \varphi_{0}$ represents the fringe order shift relative to the reference interferogram, and $\left[O P D_{\mathrm{m}}{ }^{(1)}\right]$ denotes a total of $N$ OPD values estimated from the $N$ fringe peaks. The OPD can then be defined such that the variance of $\left[O P D_{\mathrm{m}}{ }^{(1)}\right]$ is minimized, calculated by Eq. 3.40:

$$
\frac{\partial V}{\partial \psi}=\frac{\partial\left[\sum_{m=1}^{N}\left(O P D_{m}^{(1)}-\overline{O P D_{m}^{(1)}}\right)^{2}\right]}{\partial \psi}=0
$$

In an ideal case, the above variance is zero so that the right-hand side (R.H.S.) of Eq. 3.39 gives a constant $\mathbf{C}$ for any fringe order $m$ :

$$
\frac{k_{m}^{(0)}}{k_{m}^{(1)}} \cdot O P D^{(0)}+\frac{\psi}{k_{m}^{(1)}}=\mathbf{C}
$$

If an estimation error exists such that the measured phase shift becomes $\psi^{\prime}=\psi+\Delta \psi$, and the estimated OPD becomes: 


$$
O P D_{m}^{(1)}=\frac{k_{m}^{(0)}}{k_{m}^{(1)}} \cdot O P D^{(0)}+\frac{\psi}{k_{m}^{(1)}}+\frac{\Delta \psi}{k_{m}^{(1)}}
$$

By substituting Eq. 3.41 into 3.42, the estimated OPD in the presence of phase error becomes:

$$
O P D_{m}^{(1)}=\mathbf{C}+\frac{\Delta \psi}{k_{m}^{(1)}}=\mathbf{C}+\frac{\Delta \psi}{2 \pi} \lambda_{m}
$$

As a result, instead of being a constant, the estimated OPD tends to increase or decrease almost linearly with fringe-order (the $O P D_{\mathrm{m}}{ }^{(1)}$ vs. $m$ curve becomes tilted), and the slope of the tilt is given by $\kappa$ (Eq. 3.44).

$$
\kappa=\frac{O P D_{m+1}^{(1)}-O P D_{m}^{(1)}}{m+1-m}=-\frac{\Delta \psi}{2 \pi}\left(\lambda_{m}-\lambda_{m+1}\right)=\xi \cdot \Delta \psi
$$

In Eq. 3.44, the term $\xi=\left(\lambda_{\mathrm{m}+1}-\lambda_{\mathrm{m}}\right) / 2 \pi$ is a constant relating the $O P D_{\mathrm{m}}{ }^{(1)}$ vs. $m$ tilt angle and the phase estimation error. If the signal demodulation algorithm assumes the phase term $\varphi_{0}$ to be a constant, which suggests that $\Delta \varphi_{0}=0$ in Eq. 3.38, such an assumption leads to a phase error $\Delta \psi=\Delta \varphi_{0}$, and consequently, results in tilting of the $O P D_{\mathrm{m}}{ }^{(1)}$ vs. $m$ curve with resulting slope $\kappa$ defined by Eq. 3.44 .

The tilt angle $\xi$ can be obtained directly from the spectrum by identifying the wavelength positions of adjacent peaks. Figure 3-14(a) plots the calculated slope as a function of the total phase error from experimentally-obtained IFPI spectral data. After identifying the peaks in the spectrum, the total phase shift $\psi$ in Eq. 3.39 was scanned over a broad range by increasing the temperature of the IFPI sensor. Upon inserting each $\psi$ value into the R.H.S. of Eq. 3.39, a series of $O P D_{\mathrm{m}}{ }^{(1)}$ values were obtained, and $\kappa$ was calculated by linear fitting these values to the fringe order $m$.

As long as $\Delta \varphi_{0}<\pi$ and noise is sufficiently low that peak positions can be determined accurately, this erroneous assumption of constant initial phase does not cause a jump in the demodulated OPD. Under these ideal conditions, the fringe-order shift $n^{(1)}$ in Eq. 3.38 can be uniquely determined by minimizing $|\kappa|$ in Eq. 3.44 without hopping to its nearest integers. However, once $\Delta \varphi_{0}$ reaches $\pi$, two adjacent $n^{(1)}$ values generate two possible solutions for $\kappa$ with 

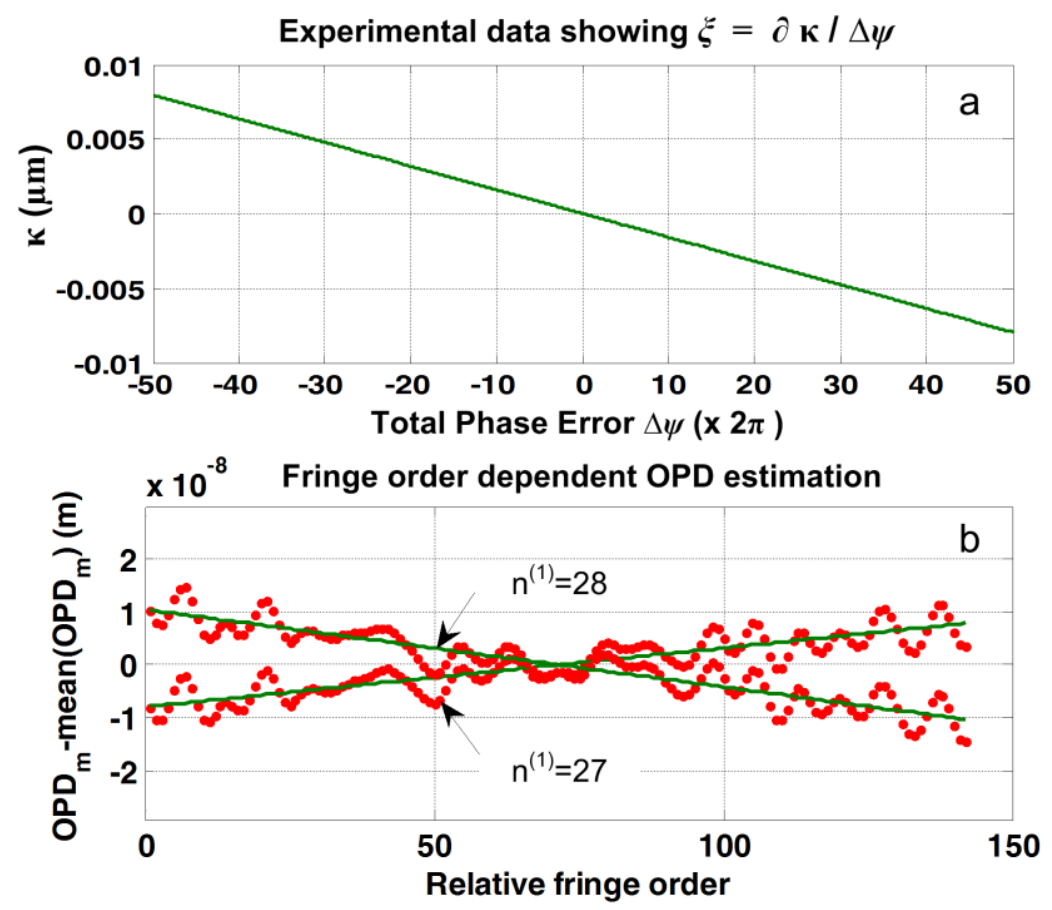

Figure 3-14. (a) Slope $\boldsymbol{\kappa}$ of the $O P D_{\mathrm{m}}{ }^{(1)}$ vs. $m$ curve as a function of phase estimation error $\Delta$ (b) An example of the tilting at a phase shift $\Delta \varphi \approx \pi$, given by the $O P D_{\mathrm{m}}{ }^{(1)}$ vs. $m$ curves for $n^{(1)}=27$ and $n^{(1)}=28$.

identical absolute values but opposite signs. This degeneracy can cause a demodulation jump to take place at the point where $n^{(1)}$ jumps to a neighboring value.

This inherent fringe order estimation ambiguity problem is demonstrated by analyzing the experimental spectral data near an OPD jump point. Shown in Figure 3-14(b), when $\varphi_{0}$ is assumed to be constant, it is impossible to distinguish whether the spectrum has shifted 27 or 28 orders from the reference spectrum. Figure 3-14(b) illustrates the relationship between the ambiguity in minimization of the slope $\kappa$ and calculating the value of fringe order shift $n^{(1)}$.

\section{b. Total phase interrogation method}

In order to avoid jumps caused by the inherent fringe-order ambiguity problem, the additional phase shift $\Delta \varphi_{0}$ in Eq. 3.38 can no longer be treated as zero. Instead, the varying phase term $\varphi_{0}(\tau)=\varphi_{0}+\Delta \varphi_{0}(\tau)$ needs to be estimated for each measurement. The following algorithm is designed specifically to achieve the required dynamic recalculation of $\varphi_{0}(\tau)$. We begin by rearranging Eq. 3.37 into the following form, in which the functional dependence on $\tau$ is explicitly 
stated and the fringe order integers are expressed such that $[m(\tau)]_{N \times 1}=[0,1,2, \ldots, N(\tau)-1]^{T}$ and $m_{0}(\tau)$ is the starting fringe-order in the spectrum.

$$
O P D(\tau) \cdot\left[k_{m}(\tau)\right]_{N \times 1}+\phi_{0}(\tau) \cdot[I]_{N \times 1}=2 \pi\left([m(\tau)]_{N \times 1}+m_{0}(\tau) \cdot[I]_{N \times 1}\right)
$$

Based on the position of each peak, multiple OPD values can be estimated, as expressed by the following equation:

$$
\left[O P D_{m}\right]_{N \times 1}=\left[c_{m}\right]_{N \times 1}+\left[d_{m}\right]_{N \times 1} \cdot Y
$$

In Eq. 3.46, we define the $[c]$ and $[d]$ vectors as: $\left[c_{m}\right]_{N \times 1}=2 \pi\left[m / k_{m}\right]_{N \times 1},\left[d_{m}\right]_{N \times 1}=\left[1 / k_{m}\right]_{N \times 1}$, and an auxiliary phase $Y=2 \pi m_{0}-\varphi_{0}$. The solution of Eq. 3.40 gives the least-square estimation of the auxiliary phase $\hat{Y}$ :

$$
\hat{Y}=-\sum_{m=0}^{N-1} C_{m} D_{m} / \sum_{m=0}^{N-1} D_{m}^{2}
$$

where $C_{m}=c_{m}-\bar{c}_{m}$ and $D_{m}=d_{m}-\bar{d}_{m}$, and $\bar{x}$ denotes the average value of $x$ (averaged over $m$ fringe orders in a single spectrum). The OPD can then be estimated from the measured total phase shift $\hat{\psi}$ based on Eq. 3.46 and Eq. 3.47:

$$
O \hat{P} D=\bar{c}_{m}+\bar{d}_{m} \hat{Y}
$$

From the auxiliary phase $\hat{Y}$, one could also estimate the additional phase term according to:

$$
\hat{\varphi}_{0}=2 \pi \cdot \text { floor }[\hat{Y} /(2 \pi)]-\hat{Y}
$$

where the function floor $(x)$ denotes rounding the variable $x$ to the nearest integer less than or equal to $x$. In practice, the estimation of $\hat{\varphi}_{0}$ and $O \hat{P} D$ can fluctuate with the same trend during measurement, which results in unwanted ripples in the measured value of $O \hat{P} D(\tau)$. This phenomenon is illustrated in the results of experimental testing of the algorithm (Figure 3-17). To eliminate this problem, we propose a significant departure from existing FP sensor Multiplexed Optical Fiber Sensors for Coal Fired Advanced Fossil Energy Systems 
interrogation techniques. Instead of monitoring changes in OPD, we define a new sensor measurement parameter, average total phase $\bar{\Phi}$, calculated by inserting measured $O \hat{P} D$ and $\hat{\varphi}_{0}$ into (2). $\bar{\Phi}$ represents the total phase at a fixed reference wavenumber, taken to be the central point in the wavenumber scan range.

$$
\bar{\Phi}(\tau)=O \hat{P} D(\tau) \cdot \bar{k}+\hat{\varphi}_{0}(\tau)
$$

As proven by the numerical simulations and experimental results in Section $\mathrm{V}$, the use of average total phase $\bar{\Phi}$ as a new measurement parameter allows for dynamic recalculation of initial phase $\varphi_{0}(\tau)$ while retaining the high measurement resolution of conventional signal demodulation methods.

c. Simulation of multimode-induced phase variation

Computer simulation was performed to verify that the total phase measurement algorithm can eliminate demodulation jumps when initial phase changes more than $\pi$. The spectrogram of an IFPI sensor was simulated using Eq. 3.28 and Eq. 3.29 with the step-index optical fiber parameters outlined in Table 3.4.

A total of 180 spectra, with an SNR of $35 \mathrm{~dB}$, were generated and evenly distributed over a temperature range from 0 to $800^{\circ} \mathrm{C}$. As the simulated temperature increased, a phase shift of about $-1.2 \pi$ was generated due to multimode excitation in the MMF. This phase shift and OPD change were calculated using the algorithm in Section 3.3.2 (b) and plotted in Figure 3-15. The blue curve in the figure shows the OPD demodulated using an algorithm which assumed constant phase. As shown in the figure, when the phase shifts over $\pi$, a series of jumps occur as a result of fringe-order estimation ambiguity. In contrast, the red curve shows the OPD calculated using the new varying phase algorithm, which is shown to remove the fringe-order-ambiguity induced OPD jumps completely. 
Table 3.4. Simulation Parameters for SMS-IFPI Sensor.

SMF core radius

SMF core refractive index

SMF cladding refractive index

MMF core radius

MMF core refractive index

MMF cladding refractive index

MMF coefficient of thermal expansion (CTE)

MMF thermo-optic coefficient (TOC)
$4.5 \mu \mathrm{m}$

1.445

$25.0 \mu \mathrm{m}$

1.448

1.434

$0.5 \times 10^{-6}\left(1 / /^{\circ} \mathrm{C}\right)$

$0.65 \times 10^{-}$

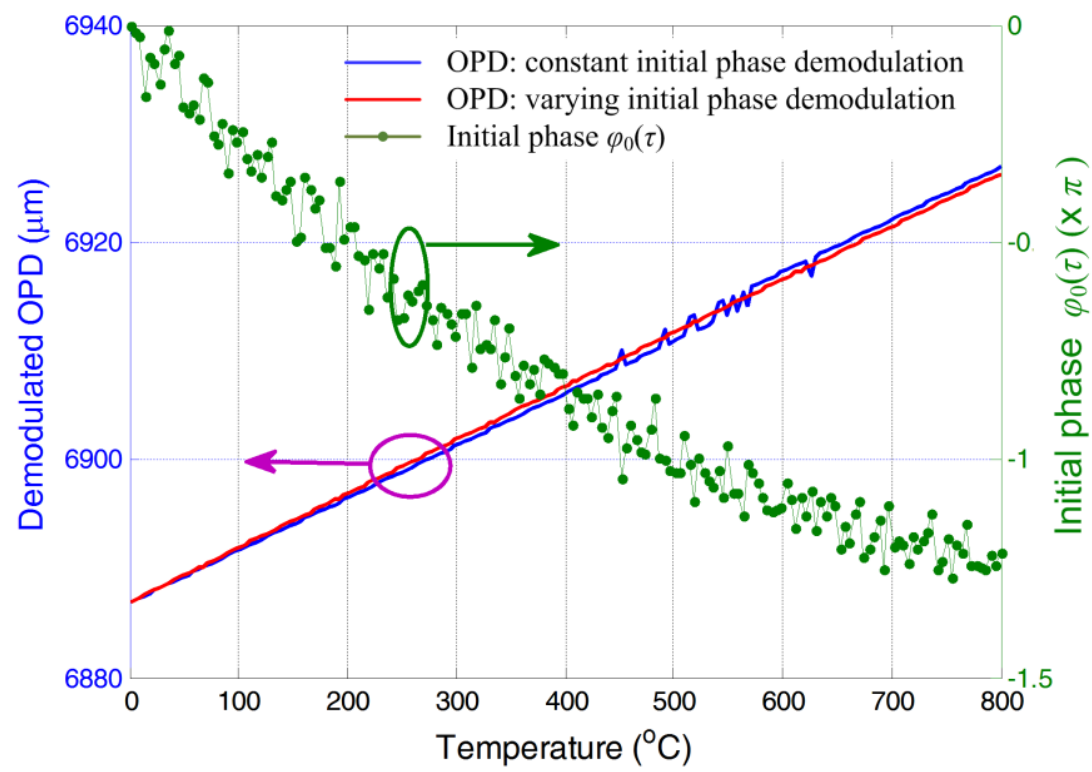

Figure 3-15. OPD of an IFPI sensor (demodulated using traditional constant phase and new total phase algorithms) and its phase shift $\Delta \varphi$ under increasing temperature. Spectral data generated by computer simulation.

\section{d. Simulated noise performance}

The performance of the total phase algorithm was further evaluated by identifying its OPD estimation accuracy at different noise levels, as shown in Figure 3-16. The evaluation method 


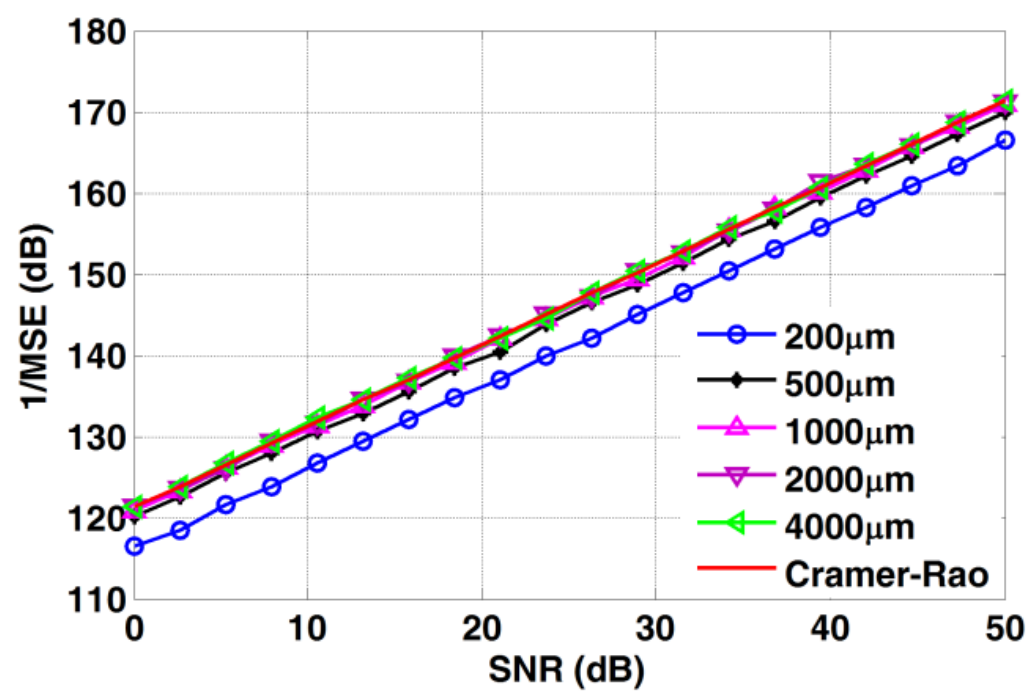

Figure 3-16. Performance of the varying total phase algorithm evaluated at different cavity lengths and SNR levels. MSE errors were calculated based on 1000 sampling points, among which phase shift from 0$1.2 \pi$ was evenly distributed. Each spectrum contains 20000 points spaced over $1520-1570 \mathrm{~nm}$.

employed was similar to that in [22]. The Mean-Square-Error (MSE) was evaluated at different cavity lengths and SNR levels by averaging over 1000 measurements at each given condition. An additional phase ranging from 0 to $1.2 \pi$ was introduced into the simulated spectra and evenly distributed over the 1000 statistical sampling points to demonstrate the robustness of the algorithm against phase drifts larger than $\pi$. The corresponding Cramer-Rao bound [25] is also plotted in Figure 3-16 to show that the varying total phase method can reach the upper theoretical bound of linear regression-based algorithms even at the presence of a phase shift as large as $1.2 \pi$.

\section{e. Experimental evaluation of algorithm}

The benefits of the varying total phase algorithm are further demonstrated by the following two experiments. In the first experiment, an IFPI temperature sensor with strong multimode excitation was used in order to show the superior performance of the algorithm in an extreme case, in which large phase shift exists due to the modal behavior of the cavity. The environmental temperature of the sensor was raised from room temperature to $600^{\circ} \mathrm{C}$.

As shown in Figure 3-17, if the phase term is assumed to be constant, a pronounced OPD demodulation jump is observed at the point where the initial phase shift exceeds $\pi$. If the variable 
initial phase algorithm in Eqs. 3.48 and 3.49 is used to demodulate the sensor, the measured OPD exhibits no demodulation jump. However, the estimated OPD exhibits a strong, quasi-sinusoidal fluctuation, which is attributed to the heavy modal-excitation in the cavity as described in [11] (not to demodulation error).

These periodic fluctuations clearly demonstrate that OPD is not a usable sensing parameter when initial phase is allowed to vary. Instead, average total phase $\bar{\Phi}$ (Eq. 3.50) is calculated to quantify changes in the IFPI sensor due to external stimuli. The result is a demodulated total phase curve that is entirely free from both jumps and sinusoidal fluctuations (Figure 3-17)

A second experiment was performed to demonstrate the advantage of the total phase algorithm under varying initial phase due to finite laser scan rate. An IFPI sensor was stressed by hanging a dead-weight onto the far-end of the fiber link with burst vibration instabilities caused by swaying of the object. These high-speed fluctuations led to chirp in the spectra due to the limited speed of the wavelength scanning in the optical detection and generated an additional phase term.

As a result, if OPD is used to characterize the sensor, the strain calibration curve (strain versus demodulated OPD) in Figure 3-18 (a) is full of small fluctuations and suffers from poor

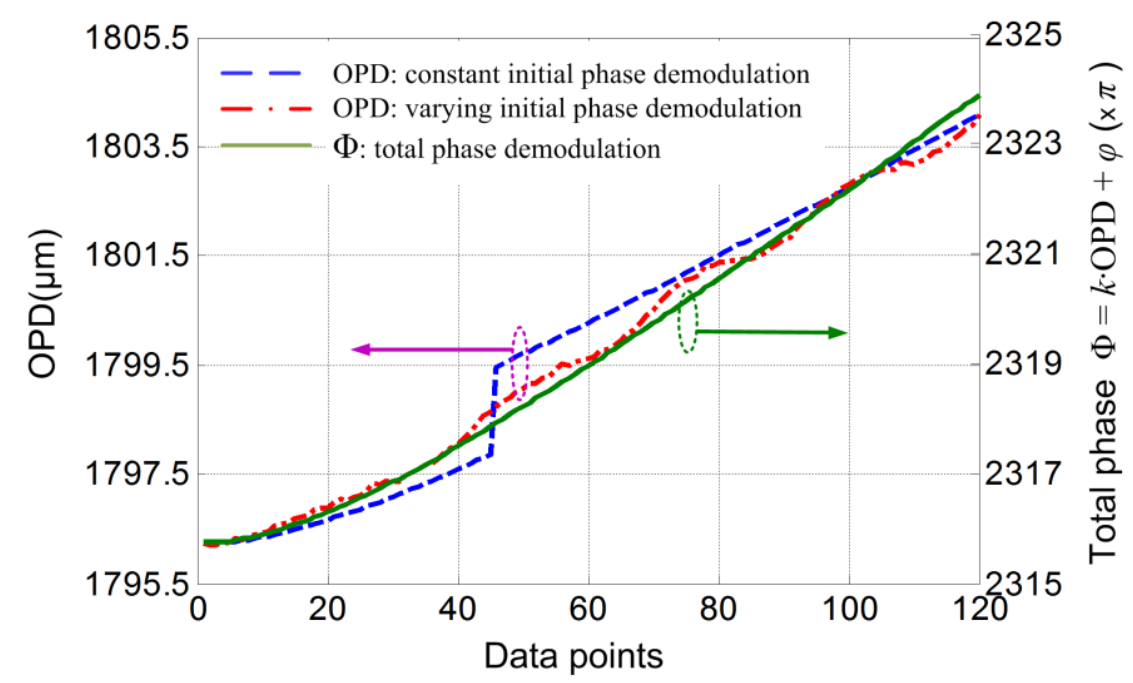

Figure 3-17. IFPI sensor OPD (demodulated using traditional constant phase and new total phase algorithms) and total phase $\Phi=k \cdot O P D+\varphi_{0}$ during an experimental controlled temperature increase. 

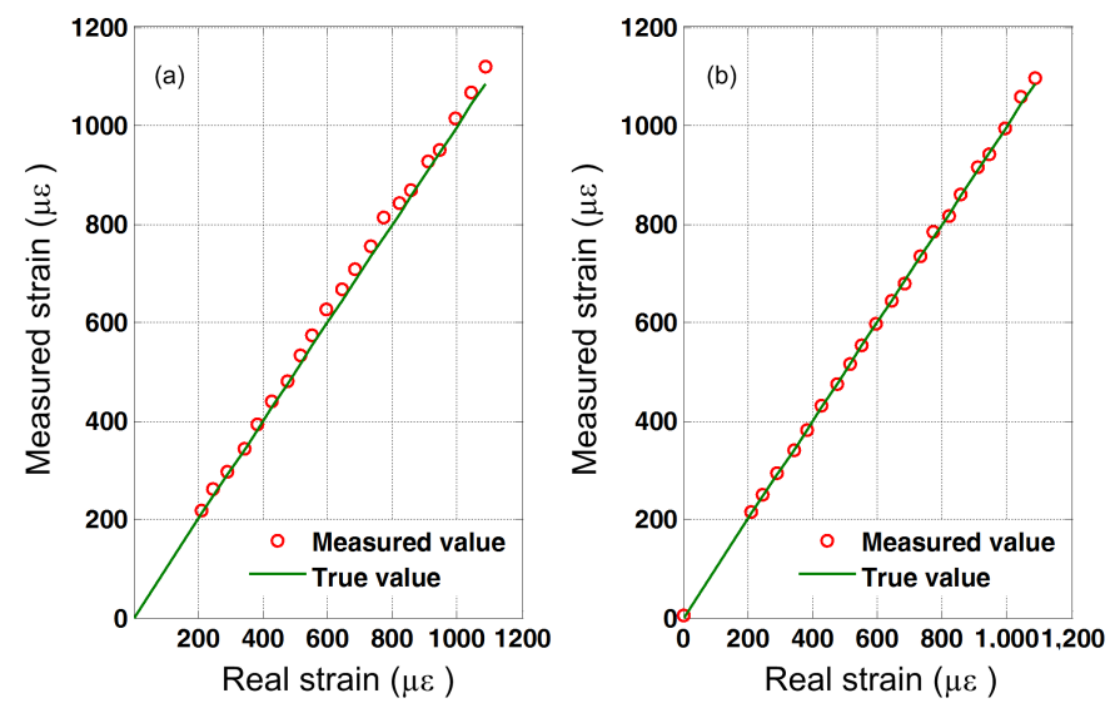

Figure 3-18. IFPI strain sensor evaluation: demodulated by (a) traditional fixed-phase OPD method, (b) new varying total phase method

repeatability. This is reflected by the large measurement error $(\mathrm{RMSE}=19.9 \mu \varepsilon)$ obtained using the traditional OPD algorithm. Instead, if the total phase is used to characterize the same fluctuating IFPI data, it results in much better repeatability and measurement accuracy $(\mathrm{RMSE}=$ $5.4 \mu \varepsilon$ ) as shown in Figure 3-18 (b).

\subsection{Sensor link design}

As discussed in Sections 3.2, by controlling the SMS-IFPI cavity lengths, the roundtrip insertion loss of each sensor can be reduced to less than $0.5 \mathrm{~dB}$. For a twenty-sensor link, this results in a total insertion loss of less than $10 \mathrm{~dB}$, which is sufficient for reliable demodulation of all the sensors. However, this requires some of the sensors to have very long cavity lengths: the twentieth sensor will have a cavity length exceeding $1 \mathrm{~cm}$. Such long cavity length tends to impose signal demodulation challenges stemming from environmental fluctuations. To address this problem, different types of MMF can be employed. Table 3.5 lists some key properties of three types of GI-MMF. The diverse optical properties of different MMFs lead to distinct pitches, allowing multiplexing of more than twenty sensors with a maximum cavity length of around $6 \mathrm{~mm}$. The designed cavity lengths should bear enough range. Figure 3-19 conceptually illustrates a potential design. 
Table 3.5. Key Properties of Three Types of MMF.

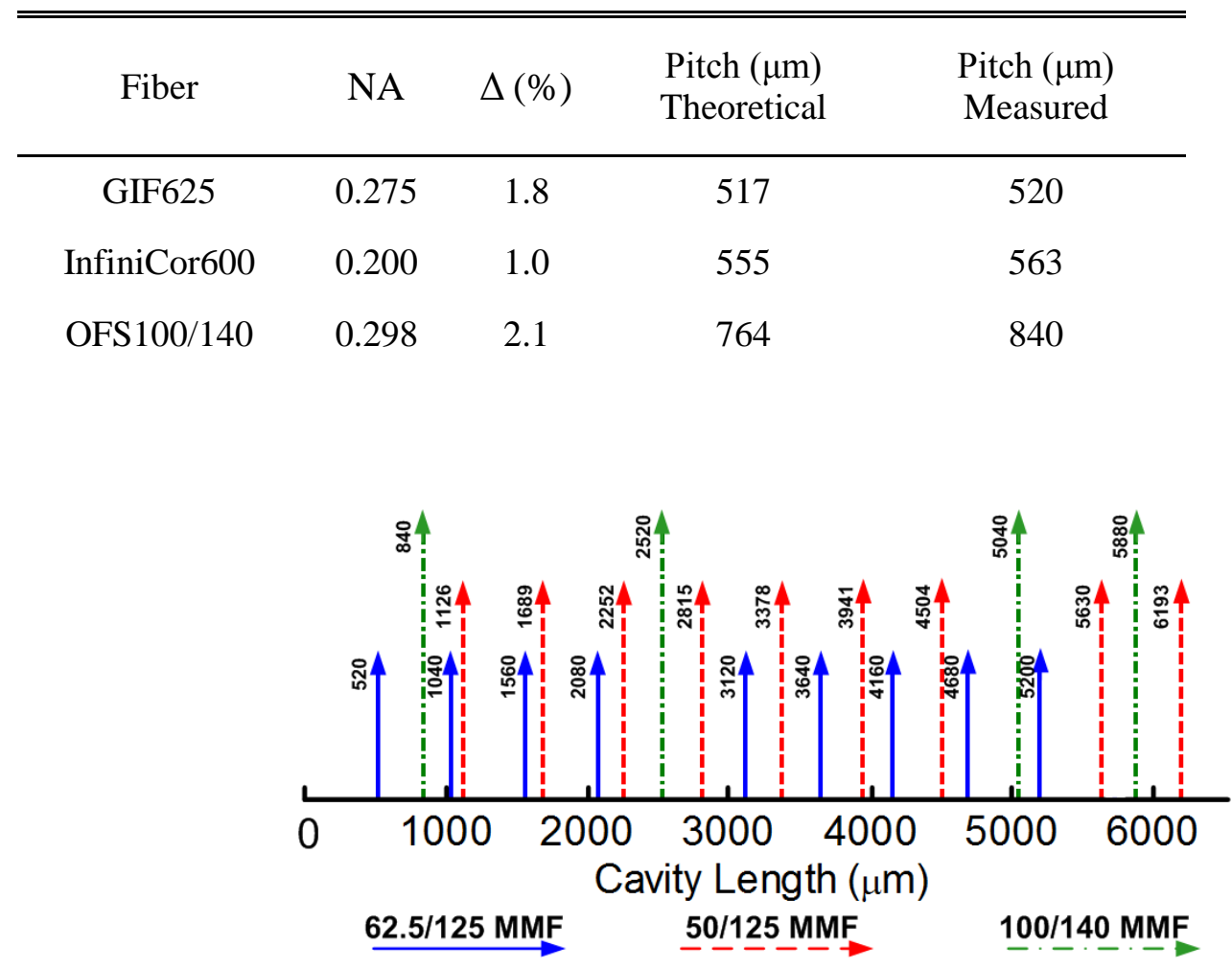

Figure 3-19. Design of a sensing link with 22 IFPI sensors multiplexed with three different types of MMF. 62.5/125 MMF: Thorlab GIF-625; 50/125 MMF: Corning InfiniCor600; 100/140 MMF: OFS-100/140. 


\section{LABORATORY TESTING OF SENSORS}

\subsection{Construction of simulated environment}

According to the requirements of the project, a simulated test environment is designed, fabricated and assembled. The test environment is capable to simulate the environment up to 800 ${ }^{\circ} \mathrm{C}$ in temperature and apply $1000 \mu \varepsilon$ in strain (in steps such as $100 \mu \varepsilon$ ). The main design is based-on a horizontal tube furnace in the Materials Science Department that provides heating capabilities and the sufficient space for sensor tests. The strain mechanism is applied to the sensor through a heavy-duty pulley that is installed on a separate stand fixed in the floor. Weights will be applied to the sensor through the wire to the plate, where the sensor is attached on. The other end of the plate is be fixed to the wall.

In order to design the ideal strain sensor test apparatus, we performed a simple calculation to determine how much a T-22 steel test plate will strain under load. The goal of this work is to design a test plate, with similar physical properties to the components in industry standard boilers, on which to attach the IFPI sensors for strain calibration and testing. The sensor

calibration requirements for the simulated testing environment include a range of 5 1000 microstrain, an accuracy 50 micro-strain, and a temperature up to $600{ }^{\circ} \mathrm{C}$. In order to keep the required weight in a reasonable range, we limit the test load to $100 \mathrm{lbs}$.

Strain is governed by the following basic equations:

$$
\begin{aligned}
& \operatorname{Strain}(\varepsilon)=\frac{\text { Stress }}{\text { Young's Modulus }(E)} \\
& \text { Stress }=\frac{\text { Force }(F)}{\text { Area }(A)} \\
& \Rightarrow A=\frac{F}{E \cdot \varepsilon}
\end{aligned}
$$

Assuming the following coefficients,

100 lbs. of weight ( $=444$ Newton $)$ 


$$
\begin{gathered}
E=2 \times 10^{7} \text { psi }=1.3789 \times 10^{11} \text { Pascal, } \\
\text { Strain }=0.001(1000 \text { micro-strain }) \\
A=444 \mathrm{~N} /\left(1.3789 \mathrm{e} 11 \mathrm{~Pa}^{*} 0.001\right) \\
A=3.219 \times 10^{-6} \mathrm{~m}^{2}
\end{gathered}
$$

We arrive at the desired dimensions for a T-22 steel test plate with square cross-section:

$$
\text { Width }=1.794 \times 10^{-3} \mathrm{~m} \text { and thickness }=1.794 \times 10^{-3} \mathrm{~m}=0.071 \mathrm{in}
$$

This 0.071 represents our "target size" for the height and width of the T-22 steel. Next, we milled the T-22 to the dimensions shown in Figure 4-1.

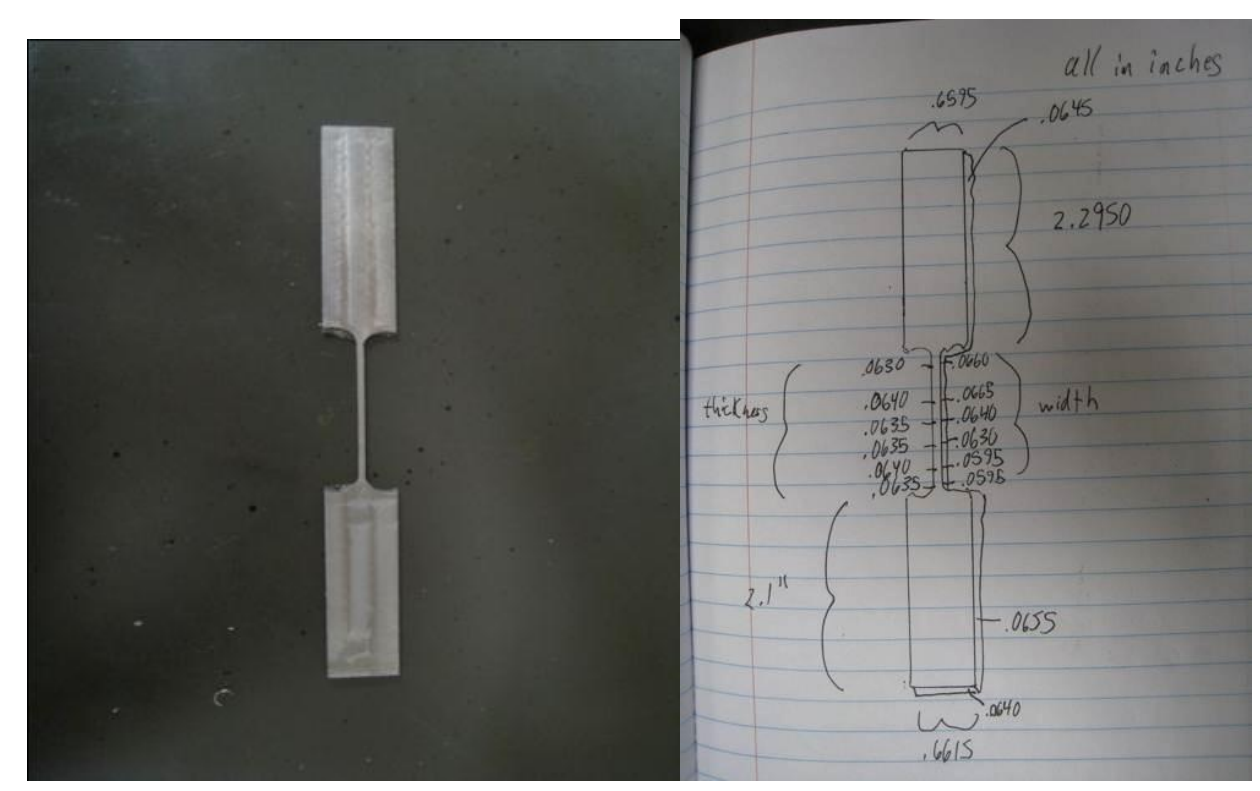

Figure 4-1. T-22 steel machined cut out (left) with dimensions seen at right.

As shown in Figure 4-1, we have fabricated a test plate with maximum and minimum crosssectional dimensions of 0.0665in and 0.0595in, respectively. The average cross-sectional thickness is $=0.06333$ in. We must now recalculate our previous example since our thinnest dimension (the point at which the steel is most likely to fail) will change the amount of force needed to obtain 1000 micro-strain in the fiber sensor. At thinnest dimension:

$$
A=0.0595 \text { in } * 0.0635 \text { in }=3.77825 \mathrm{in}^{2}=\mathbf{2 . 4 3 7 5 8 0 9 3} \times 10^{-6} \mathrm{~m}^{2}
$$


When plugged into the previous equation:

$$
\begin{aligned}
& A=F /(E . \varepsilon) \text { Solve for } F(F=?), E=2 e 7 p s i=1.3789 e 11 P a, \text { Strain }=0.001 \\
& 0.095967 \mathrm{~m} 2=F /(1.3789 e 11 P a * 0.001)
\end{aligned}
$$

Thus, a force of $\mathrm{F}=336.12 \mathrm{~N}=75.56 \mathrm{lbs}$ to obtain 1000 micro-strain in the $\mathrm{T}-22$ steel.

The simulated testing environment is composed of 4 components:

- Tube furnace

- Strain sample and strain clamps

- Strain weights, support stand for applying strain via weight

- And a cable anchor point and cable that tie all the pieces together.

The tube furnace has a high temperature capability of $1800^{\circ} \mathrm{C}$ with a 3-inch outside diameter tube and a heat zone of approximately 12 inches. The overall configuration uses an existing wall to support one end of the cable while the other end is attached to a clamp that holds one end of the strain sample. On the other end of the strain sample an identical clamp is secured. At the end of the second clamp a cable is attached with the other end of the cable running over a supported pulley and attaching to a sled that can have weight added in order to increase the strain on the sample. An overall schematic of the system is shown in Figure 4-2. 


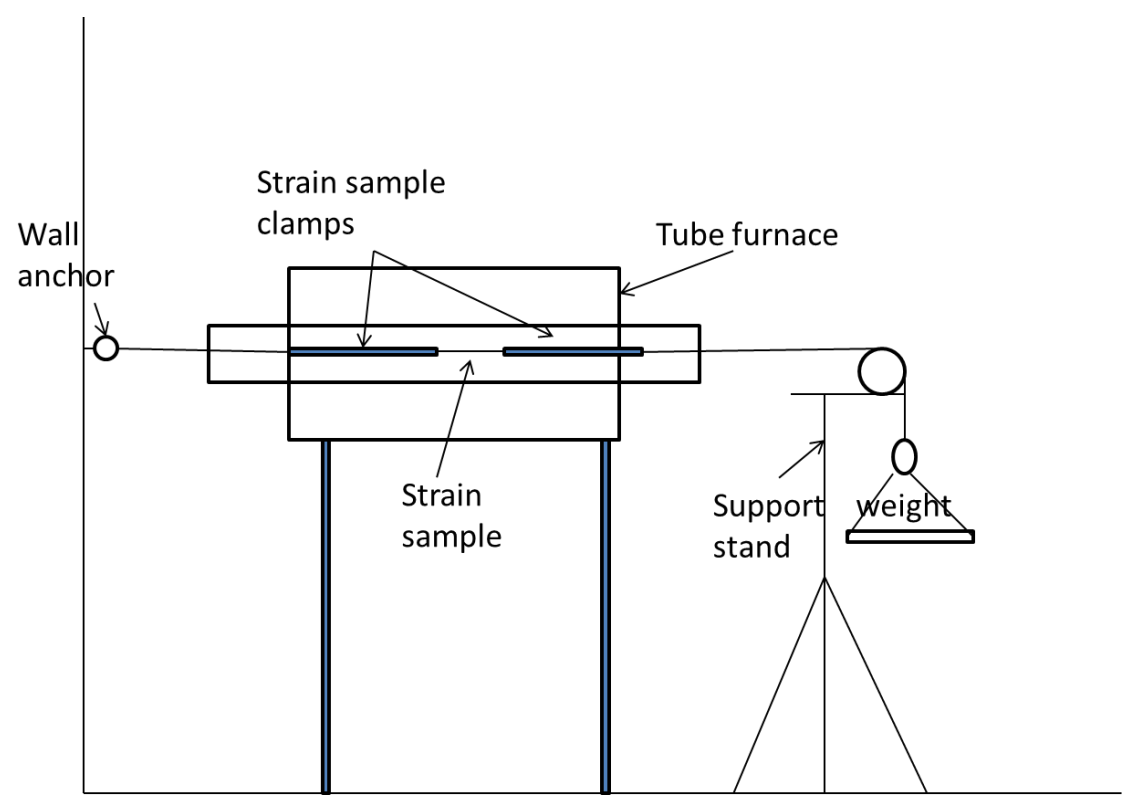

Figure 4-2. Schematic of simulated testing environment

The strain sample is held in the furnace with a pair of mechanical clamps made out of the same material (T-22 stainless steel) as the strain sample. Two lengths of T-22 bar stock were prepared by milling out a slot at one end of the bar to a depth of 0.070 " for a length of approximately 2". A 2.5 " piece of T-22 bar stock was welded to the top of the slotted bar stock so that it protrudes over the slotted portion of the bar. This configuration acts as the clamping surface. At the end of the welded on portion and the bar immediately below it, a flat piece of steel was welded on with the lower piece threaded for a $5 / 16$ " bolt and the upper portion drilled so that the bolt slides through freely. The attached pieces provide the clamping force for holding the strain sample in place. Attaching the clamp to the cables is done by connecting a cable to a French link connector that is inserted into a hole drilled at the end opposite the clamp. Figure 4-3 shows the clamp portion of the configuration. 


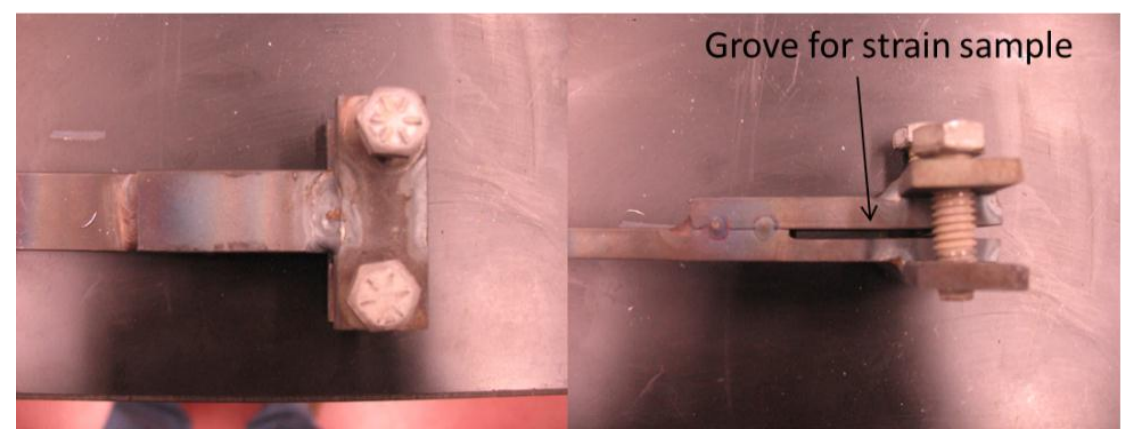

Figure 4-3. Strain clamp mechanism.

The position of the strain sample in the clamps requires the alignment of the clamps in addition to securing the clamps while the sample is being clamped into position. This is taken care by the fabrication of a jig that holds both strain clamps while allowing the strain sample to be inserted. A jig was made by welding a 1" square tube onto a longer 2" wide piece of steel bar. Onto the steel bar 4 small steel pieces were welded that had threaded matching holes to a similar steel piece. This configuration acts as a clamping mechanism to hold the 2 strain sample clamps. During installation of the strain sample the fixture can be clamped down onto a surface to fix the fixture in place. In addition to holding the strain clamps during installation of the strain sample the jig will also be used for transport of the assembly during installation of the IFPI strain sensors onto the strain sample. The jig is shown in Figure 4-4 and the fixture during installation of the strain sample is shown in Figure 4-5. 


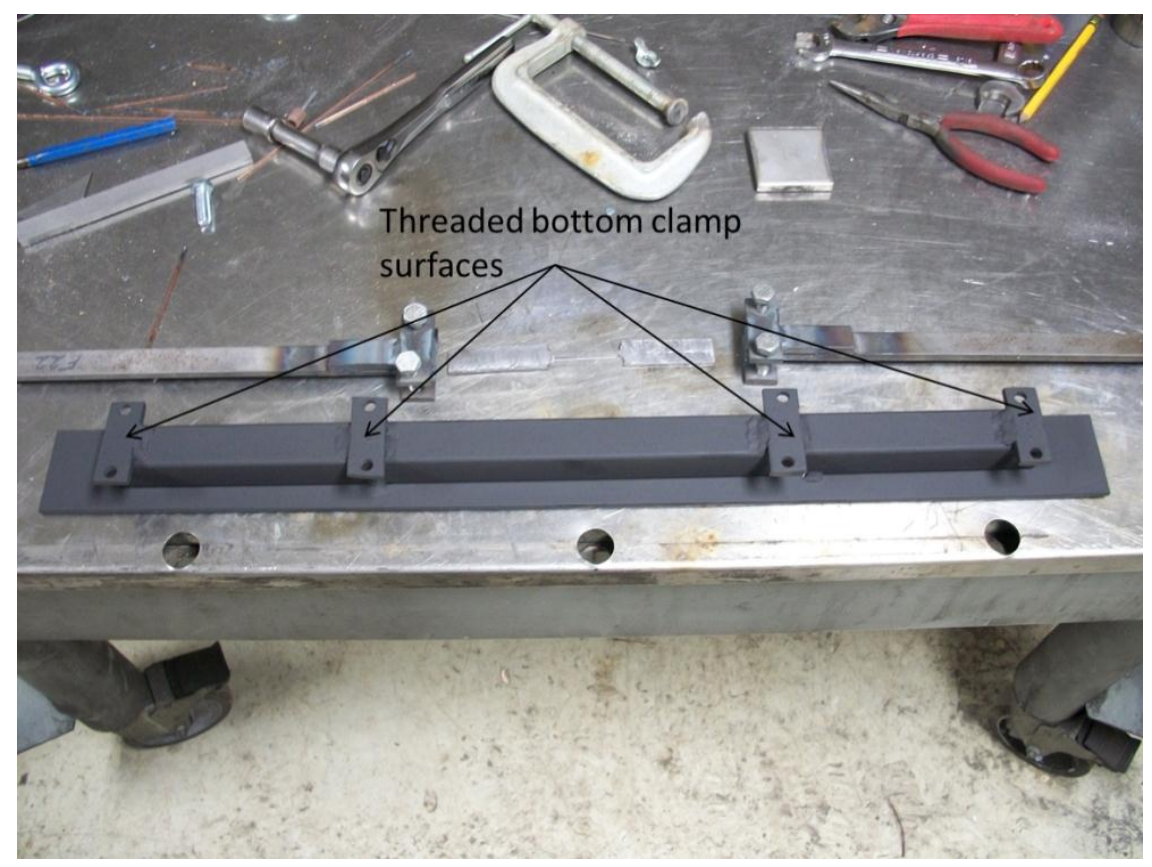

Figure 4-4. Strain sample and clamp mounting jig.

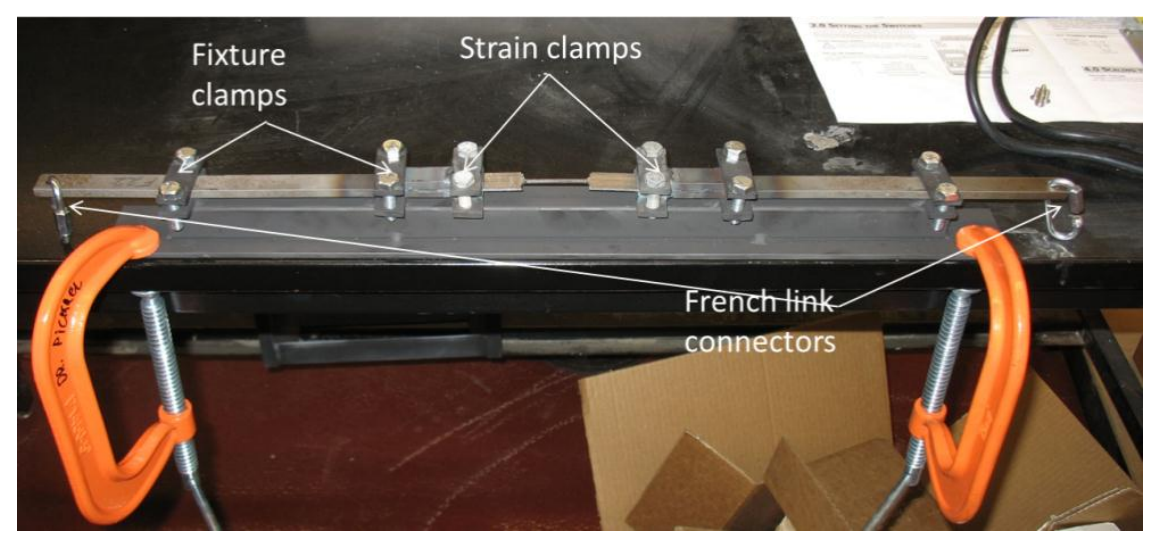

Figure 4-5. Mounting jig during installation of strain sample.

The weight stand was made from $1 / 8$ " and 3/16" steel angle welded together into a structure that allows for free hanging weight to apply strain to the sample through an attached cable. In order to make the weight hang freely an arm was made which the pulley sits on so that the cable routes over into free space. The arm assembly is threaded onto a $3 / 4$ " diameter threaded rod that allows for the height of the pulley to be adjusted as necessary. Since the pulley is supported on a rod the adding of weight will cause a deflection in the rod and alter the actual strain in the sample. Prevention of the deflection was accomplished by adding a support to the rear of the arm to counter act the force due to the weight. The support was constructed out of threaded rod 
welded to brackets that attach at the arm and the base with a turnbuckle in the middle to adjust length and tension applied. Holding the weight is done with a box made from welded steel angle. The corners of the box were drilled and cable was run from corner to corner and looped in the middle to have a place to attach a hook. An image of the stand and the weight box is shown in Figure 4-6.

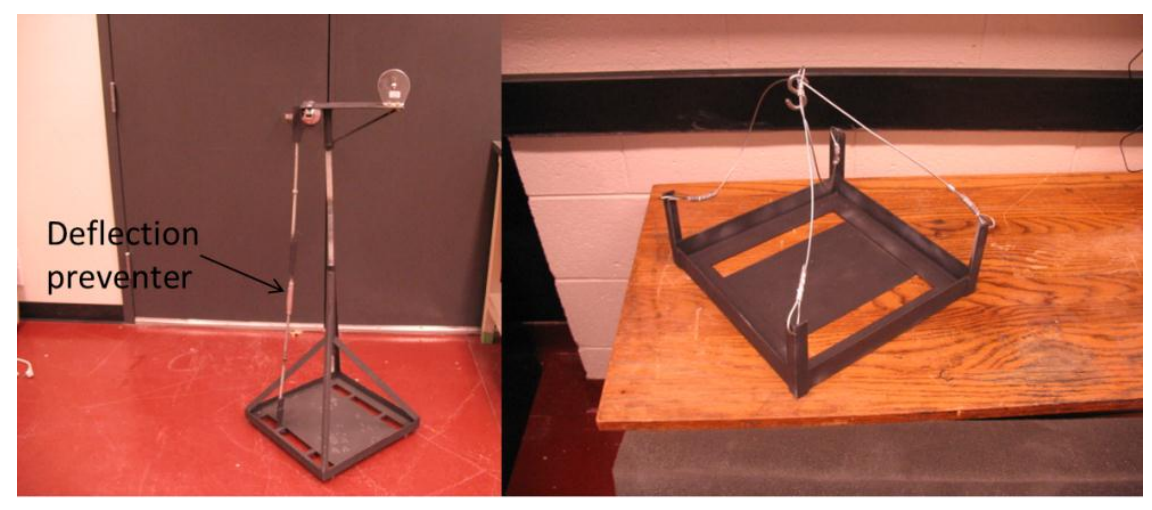

$\begin{array}{ll}\text { a) Weight stand } & \text { b) Weight box }\end{array}$

Figure 4-6. (a) Weight support stand and (b) weight box.

Attaching of the individual pieces together was done with 3/32" cable in which a loop was made at each end of the cable segment and secured by swaging. There are two lengths of cable in the system, one from the wall to one strain clamp and one from the other strain clamp to the weight box. The completed installation into the furnace with all the components is shown in Figure 4-7. 


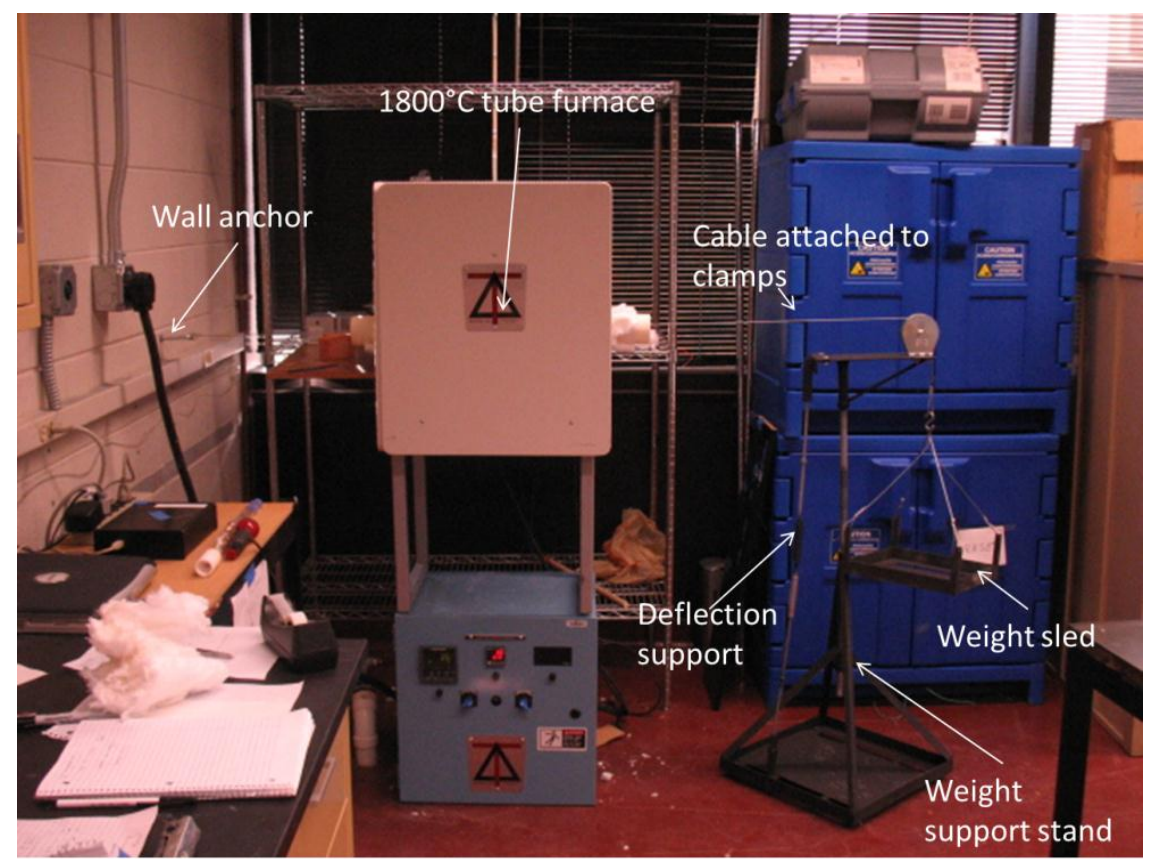

Figure 4-7. Complete assembly of simulated testing environment.

\subsection{Strain sensor attachment investigation}

\subsubsection{Sensor response using the direct bonding technique}

Although theoretically, the glass fiber can withstand the strain up to $10^{5}$ microstrain, the sensors are made by splicing sections of fibers together, hence the mechanical strength is weaker than the original fiber. The resultant sensors have quite different tensile strengths. Especially, when adhesive is used to attach the sensors on the metal surface, during the curing process, strains from various random directions are applied to the sensors and bare fibers. Sensors and fibers can be easily broken at much smaller strains than the theoretical breakage values. An example is given in the following section.

The Alstom waterwall tube (T23) has a CTE between 11.3 and 13.9 microstrain $/{ }^{\circ} \mathrm{C}$, in the temperature range of $20 \sim 600{ }^{\circ} \mathrm{C}$, according to the technical document from Alstom Power Inc. therefore, the thermal strain produced by this material can be up to 8120 microstrain. The experiment shows the sensor failed at $350{ }^{\circ} \mathrm{C}$, as in Figure 4-8. 


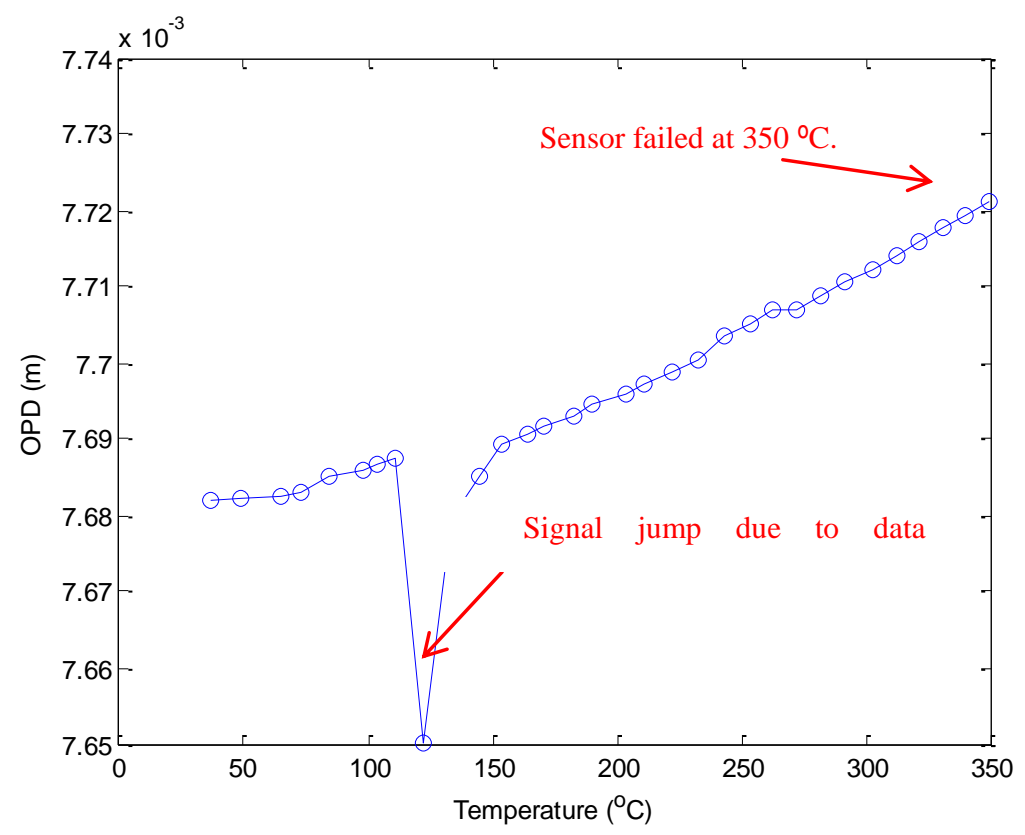

Figure 4-8. A sensor response from a direct bonding attachment. The sensor failed around $350{ }^{\circ} \mathrm{C}$.

At $350{ }^{\circ} \mathrm{C}$, the strain applied to the sensor shouldn't exceed the breakage value. However, spectrums from lower temperatures show that the sensor was under strains from directions other than the lateral direction. Therefore, the spectrum is seriously distorted, as seen in Figure 4-9(b) when the sensor is at temperatures of 111,122 and $133^{\circ} \mathrm{C}$.

(a)

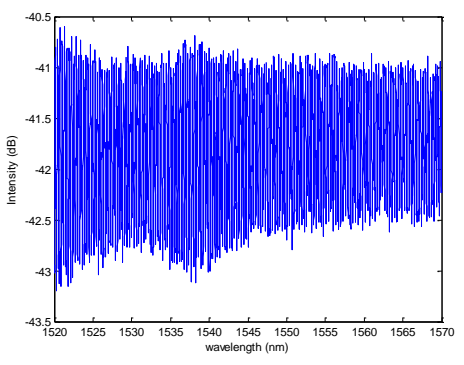

(b)

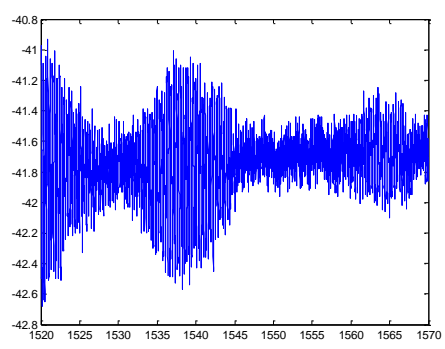

(c)

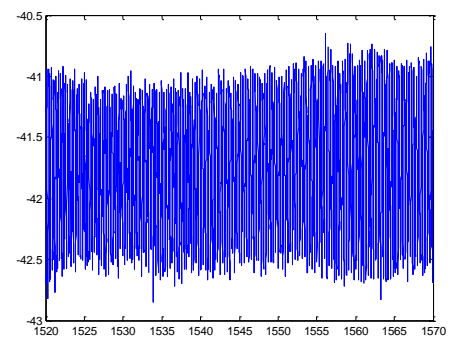

Figure 4-9. Sensor spectrums at 111,122 and $133{ }^{\circ} \mathrm{C}$. 
Similar experiments were conducted and sensors failed at different temperatures. However, the failures may be due to various reasons, especially for the alumina-based adhesive we used (Cotronix 989 FS), it expands and shrinks during the curing, heating and cooling processes and is a huge threat to the delicate fiber and sensor during the experiment. We found that breakages happened mostly at the bare fiber segments.

One solution to prevent the sensor breakage at the bare fiber section is to use gold-coated fibers to fabricate the sensors. The gold-coated fibers can be mechanically strong up to $700{ }^{\circ} \mathrm{C}$. Such fiber material will significantly reduce the unwanted fiber breakage during the attachment and testing process.

\subsubsection{Sensor bonding to a secondary material}

In order to reduce the strain transferred from the T23 alloy, our approach is to attach the sensor to a secondary material that has a lower Coefficient of Thermal Expansion (CTE) and a high Young's modulus than those of the stainless steel (T23, CTE 11 14 $\mu \varepsilon /{ }^{\circ} \mathrm{C}, \mathrm{E} 160 \sim 208 \mathrm{GPa}$ ), and then attach this material to the steel surface. From our investigation in the last quarter, we have used alumina plate for this purpose, whose $\mathrm{CTE}$ is $\sim 5 \mu \varepsilon /{ }^{\circ} \mathrm{C}$ and $\mathrm{E}$ is $300 \mathrm{GPa}$. The sensor attachment is shown in Figure 8, where a sensor is attached on an alumina plate with the same adhesive (Cotronix 989 FS) and then onto the waterwall tube. 

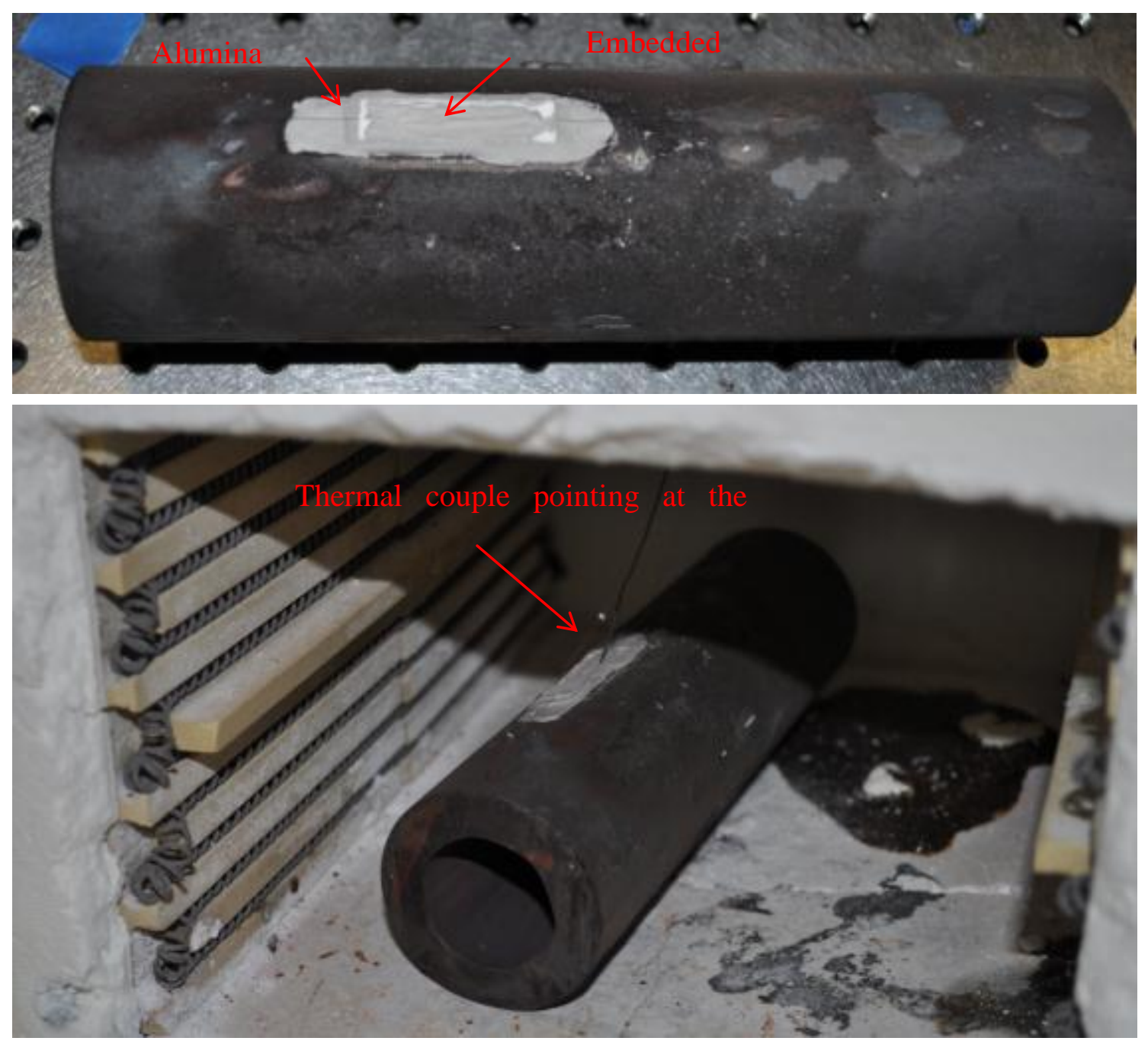

Figure 4-10. Sensor attachment and testing.

The sensor is then placed into the furnace with a thermal couple pointing right at the sensor for the temperature reference, as shown in Figure 4-10. The whole tube is heated to $650{ }^{\circ} \mathrm{C}$ at a rate of $2{ }^{\circ} \mathrm{C} / \mathrm{min}$, and then cooled naturally back to room temperature. The optical pathlength difference (OPD) due to the thermal expansion of the sensor is measured with respect to the temperature. The sensor response is shown in Figure 4-11. 


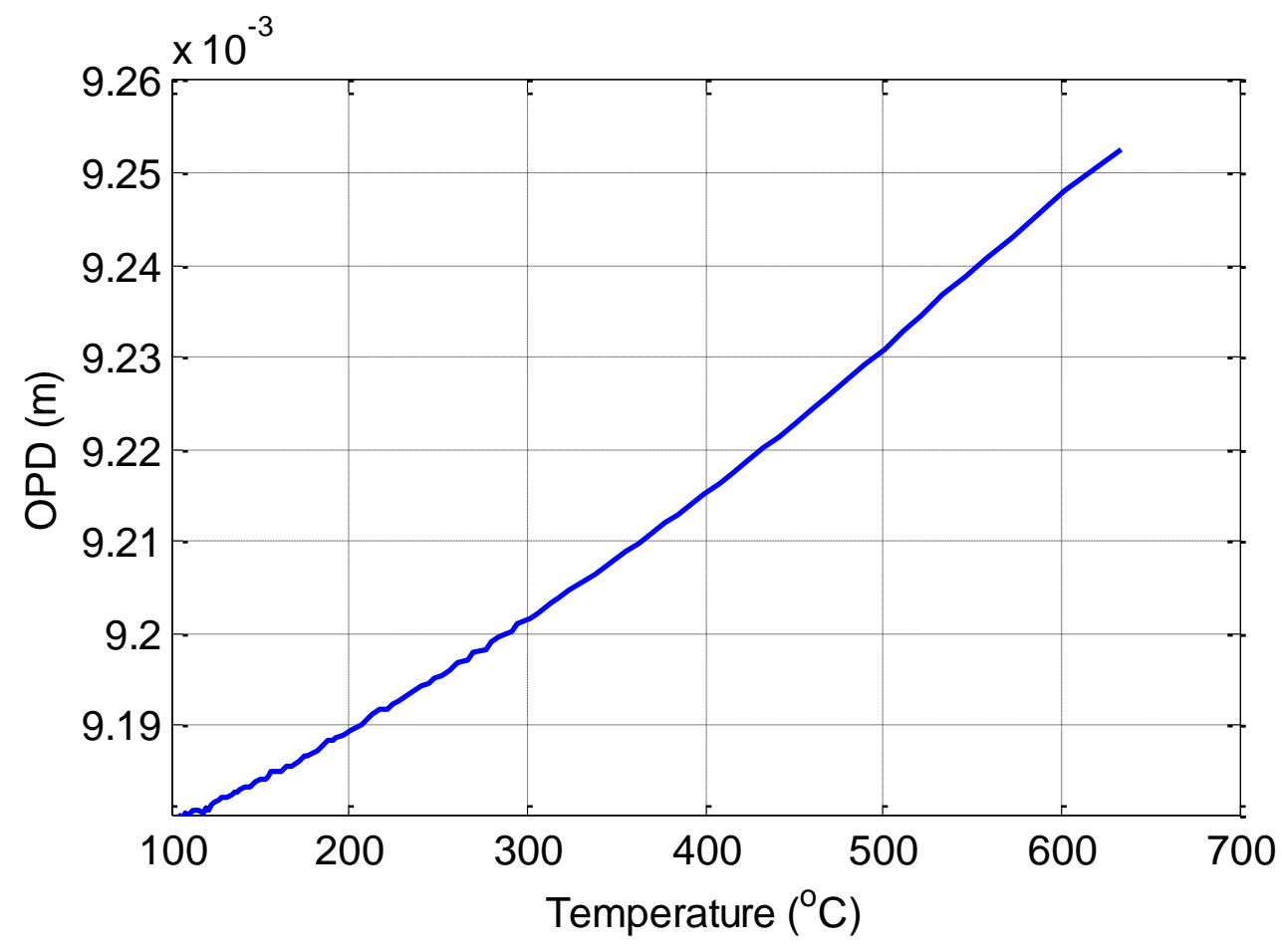

Figure 4-11. Sensor response to $650{ }^{\circ} \mathrm{C}$.

From this experiment, by using previous sensor calibration date between the strain and the Optical Path difference (OPD) due to temperature (dT), we can calculate the total measured strain $/{ }^{\circ} \mathrm{C}$ coefficient is: $\mathrm{C}=\mathrm{d}_{\mathrm{OPD}} / \mathrm{dT} / \mathrm{OPD} \sim=15 \times 10^{-6} /{ }^{\circ} \mathrm{C}$;

Since the glass fiber expands itself during the heating process, fiber CTE and Thermal-optic coefficient $(\mathrm{dn} / \mathrm{n} / \mathrm{dT})$ together are: $\mathrm{C}_{\text {fiber }} \sim 9.3 \times 10-6 /{ }^{\circ} \mathrm{C}$ (measured from previous temperature calibration data).

Thus, we have the measured strain coefficient from the substrate: $\mathrm{C}_{\text {substrate }}=\mathrm{C}-\mathrm{C}_{\text {fiber }}$, so the $\mathrm{C}_{\text {substrate }} \sim 6 \times 10^{-6} /{ }^{\circ} \mathrm{C}$, which is greater than alumina's CTE $5 \times 10^{-6} /{ }^{\circ} \mathrm{C}$. This result is reasonable for bonding a sensor on an alumina plate.

From the experiments described in the above sections, bonding alumina plate to a metallic surface using adhesive did not give the best performance when they are heated to high tempeatures and expand due to thermal expansions. Therefore, a mechanical fixture is designed and fabricated in order to bond the alumina plate and transfer the thermal strain from the T-23 test plate to the sensor, as shown in Figure 4-12. The sensor was attached to an alumina plate. 
Then the plate was clamped by a set of brackets through four screws on each side, where the brackets were also fixed to the T-23 test tube through two screws.
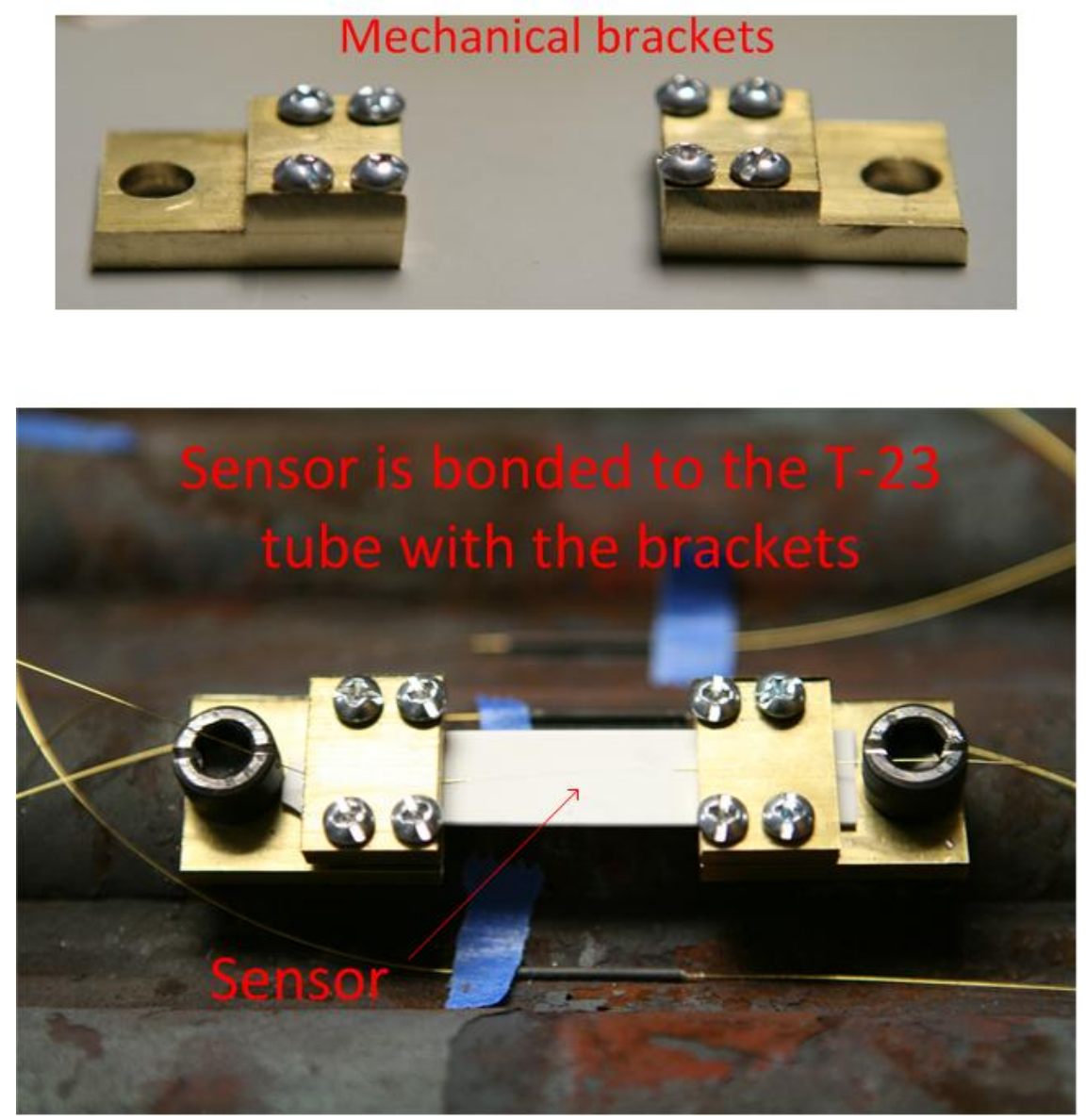

Figure 4-12. Sensor bonded to the T-23 tube by a set of mechanical brackets.

\subsubsection{Strain sensor compensation technique}

Although bonding the sensor to the secondary material will reduce the amount of strain in the sensor as introduced and tested in the previous sections, this technique still requires bonding ceramics and metals. Such a bond under high temperatures could be a huge challenge as well as under pressure and corrosive environments, such as inside a commercial boiler. Thus, we are also investigating a technique that can be used in a harsh environment without (or least) using adhesives. A compensation technique using a large CTE material than the T-23 alloy is proposed. Theoretically, this technique can achieve zero strain in the sensor. 


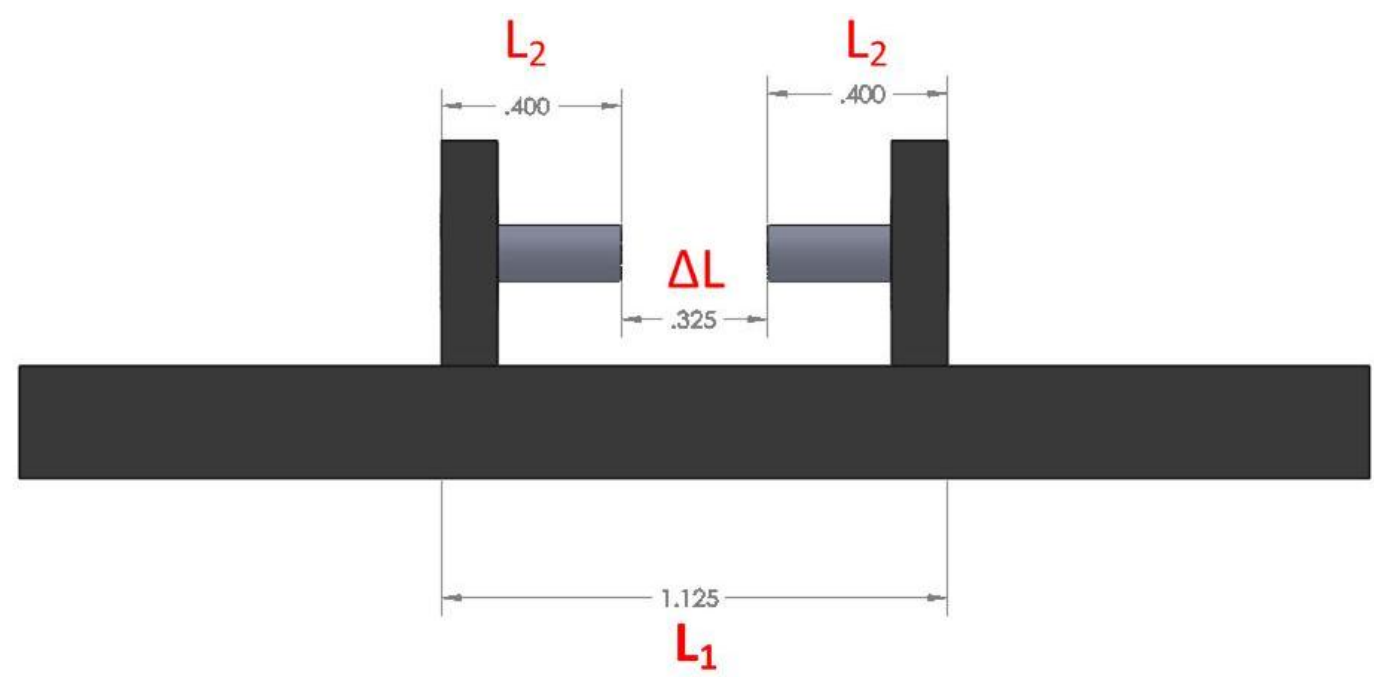

Figure 4-13.The compensation technique for reducing the strain in the sensor.

The compensation technique is illustrated in Figure 4-13, where the dark material is the T-23 alloy and grey material is the compensation material. It is important to let $\mathrm{C}_{2}>\mathrm{C}_{1}$

Coefficient of thermal expansion (CTE)

Base materials (T23) $\mathrm{C}_{1}$

Compensation material $\sim \mathrm{C}_{2}$

When the whole assembly is heated by temperature $\Delta \mathrm{T}$, it is under thermal expansion:

$$
C_{1} \Delta T L_{1}=2 C_{2} \Delta T L_{2}
$$

In the meantime, the total length (suppose zero expansion in the sensor section):

$$
L_{1}=2 L_{2}+\Delta L
$$

Therefore,

$$
L_{1}=\frac{2 \frac{C_{2}}{C_{1}} \Delta L}{2\left(\frac{C_{2}}{C_{1}}-1\right)}
$$




$$
L_{2}=\frac{\Delta L}{2\left(\frac{C_{2}}{C_{1}}-1\right)}
$$

The proposed assembly dimensions can be calculated by knowing $\mathrm{C}_{1}, \mathrm{C}_{2}$ and $\Delta \mathrm{L}$. From the expression of $\mathrm{L}_{1}$ and $\mathrm{L}_{2}$, we can conclude that $\mathrm{C}_{2}>\mathrm{C}_{1}$, in order to make the compensation work.

For example, given $\mathrm{C}_{1}=14$ for $\mathrm{T} 23$ alloy, $\mathrm{C}_{2}=20$ for copper, and $\Delta \mathrm{L}=0325$ in, we can calculate: $\mathrm{L}_{1}=1.08$ in and $\mathrm{L}_{2}=0.34$ in.

There are several possible candidate materials with larger CTEs than the T-23 alloy, such as aluminum. However, this project requires temperature measurements up to $700{ }^{\circ} \mathrm{C}$, which exceeds the melting point of aluminum. Therefore, we chose copper in the current stage of research.

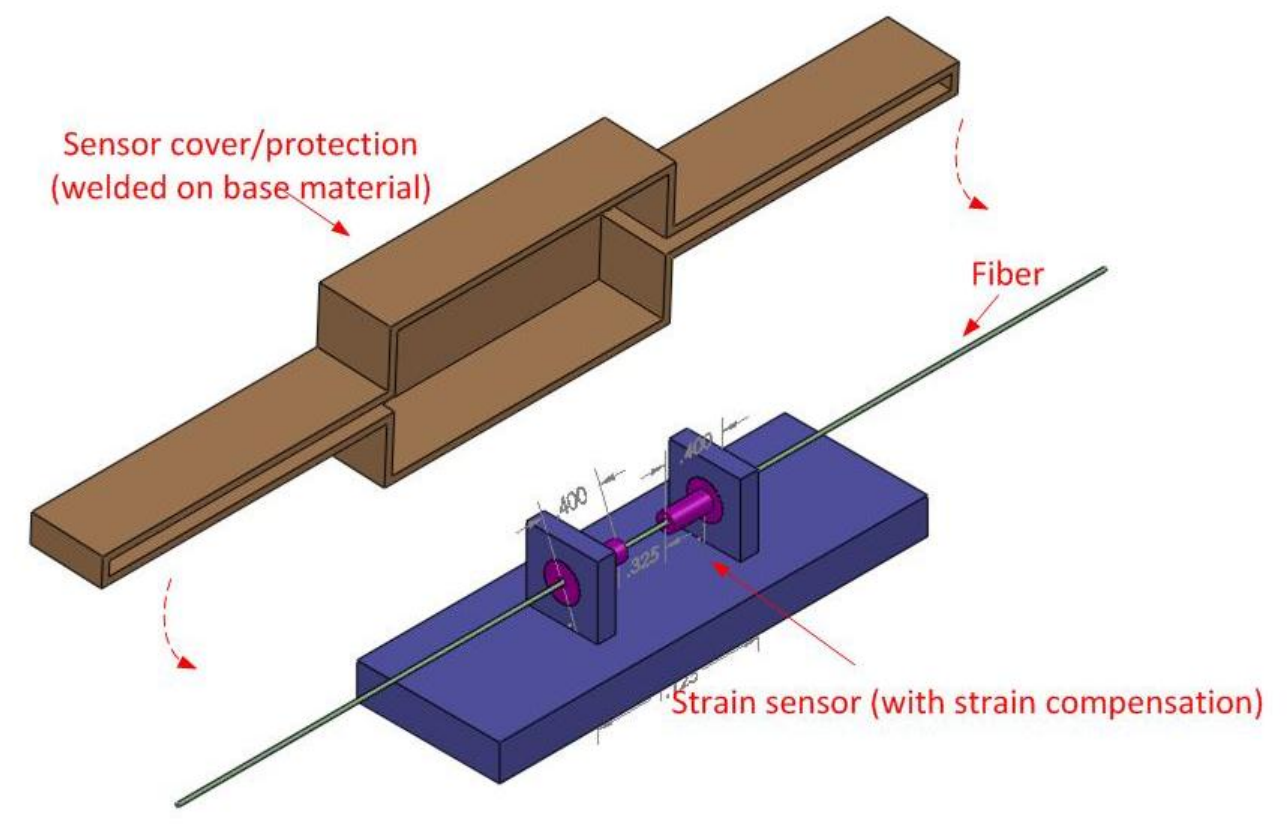

Figure 4-14. The sensor assembly when using in a harsh environment.

As illustrated in Figure 4-14, the sensor will be attached through the copper material with the sensor section in the middle. The copper and the T-23 alloy will be welding together with calculated dimensions. When this assembly is used in a harsh environment, a protective cover 
can be designed to protection the sensor assembly from the outside environment. Welding can be used as a more reliable technique than bonding with adhesives.

\subsection{Temperature sensor protection tube welding}

As proposed previously, a distributed sensor link containing five temperature sensors and one strain sensor will be deployed on the waterwall sample. Therefore, for the five temperature sensors, protection tubes are needed at these specified locations. We have found an outside vendor who can weld these tubes using laser welding technique and shows very high quality welding. A detailed drawing for the tube positions can be seen from Figure 4-15.

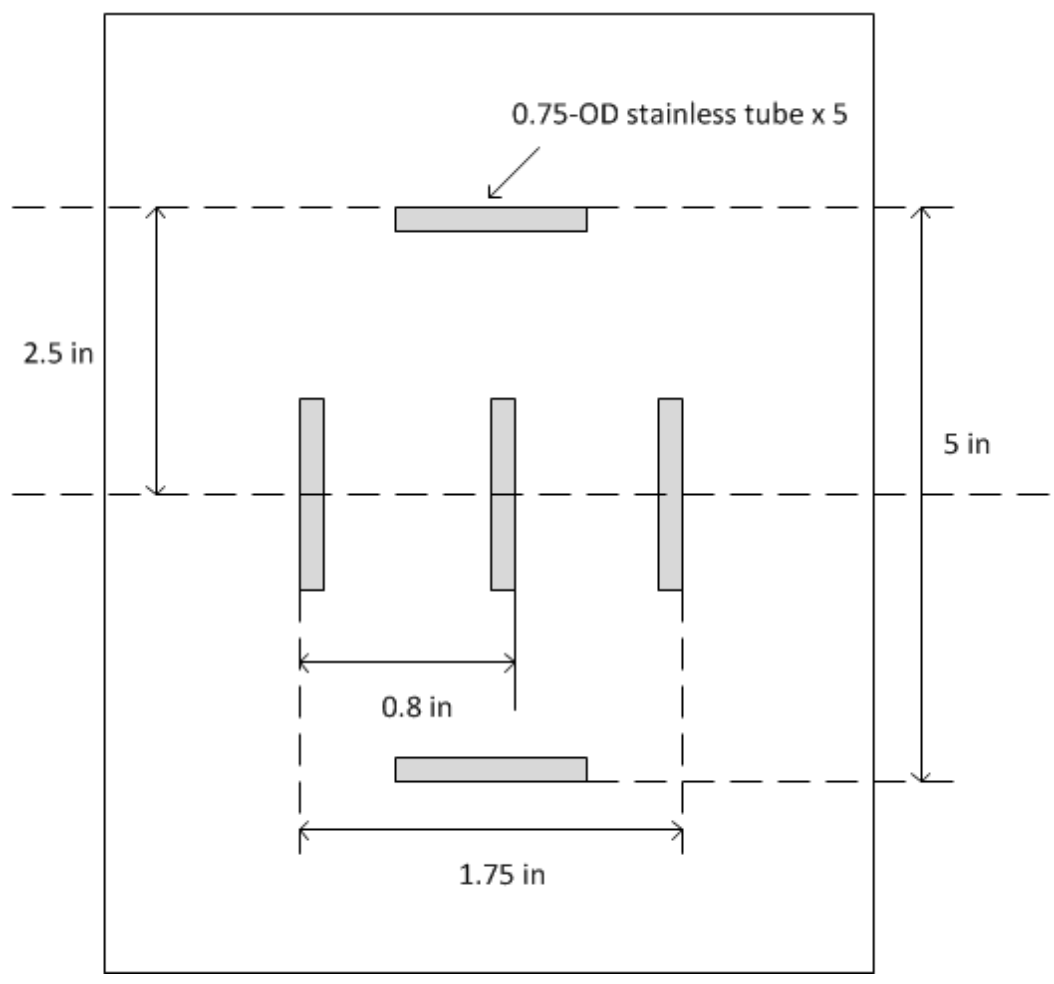

Figure 4-15. A drawing for temperature sensor protection tubes.

\subsection{Testing system fabrication/preparation}

The test plate was obtained from Alstom power Inc. It's a section of waterwall tube made of T23 alloy. As required in the specifications, five temperature sensors and one strain sensor were fabricated in one sensor link and attached to the test plate at the locations specified in Figure 4.15. In order to increase the overall mechanical strength and stability under of the fiber/sensor 
link under high temperatures, all sensors were fabricated in gold-coated fibers. The test plate with the sensor link was placed in a furnace for temperature calibration. The strain sensor was calibrated separately with a setup shown in Figure 4-16.

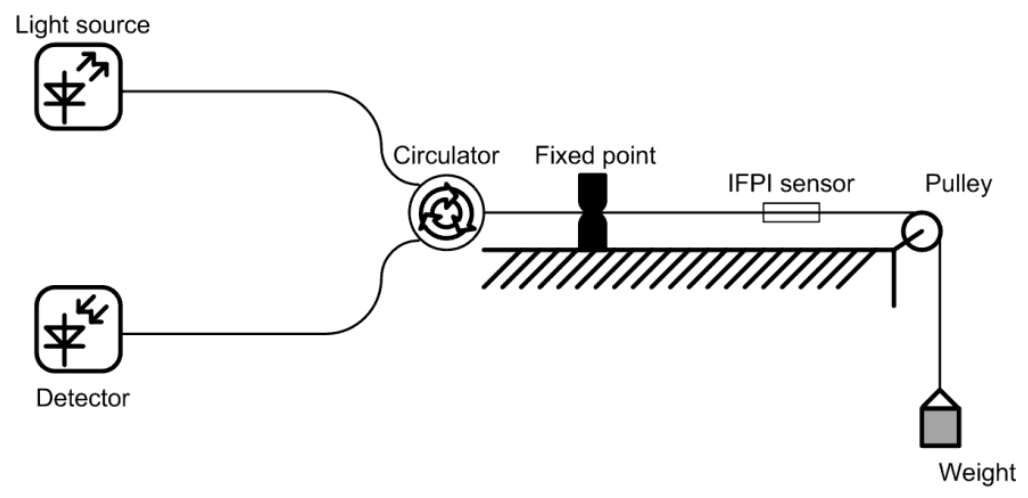

Figure 4-16. Strain calibration setup.

In the calibration procedure, we applied different weights to the fiber to calibrate the sensor from $0 \mu \varepsilon$ to more than $1000 \mu \varepsilon$. At each weight value, the interference pattern of the sensor was captured and its corresponding OPD and total phase calculated.

\subsection{Test results}

\subsubsection{Some signal processing remarks}

\section{a. Choice of sampling rate}

The system used for interrogating the sensor chain was Component Testing System (CTS, Model Si-720, Micron Optics, Inc.), which had a wavelength resolution of $5 \mathrm{pm}\left(10^{-12} \mathrm{~m}\right)$, covering spectral range from 1520 to $1570 \mathrm{~nm}$ at 20,000 sampling points and a dynamic range of $-70 \mathrm{~dB}$. The system had two choices for sampling rates, $5 \mathrm{~Hz}$ and $0.5 \mathrm{~Hz}$. The choice of sampling rates depends on the specific application, as will be discussed.

In addition to modal excitation, finite sampling rate of the spectrometer also contributes to varying initial phase. Assuming the OPD of the sensor changes with speed $R(\mathrm{~nm} / \mathrm{second})$ and it takes the data acquisition unit $S$ seconds for a single scan of the probe wavelength over the 
spectral range, the OPD change during each scan is given by $\triangle O P D=R \cdot S[\mathrm{~nm}]$. The expression for total phase is accordingly modified to:

$$
\Phi(k)=\left[O P D_{1}+\frac{R \cdot S}{\Delta k}\left(k_{1}-k\right)\right] k+\varphi_{0}
$$

where $O P D_{1}$ and $k_{1}$ are the OPD and wavenumber at the beginning of each scan, $\Delta k$ is the spectral range of the spectrometer, and $\varphi_{0}$ represents the initial phase term generated by any other effects. The variation in initial phase $\Delta \varphi_{0}$ caused by the sampling can be calculated from Eq. 3.44, while the L.H.S. of Eq. 3.44 can be well estimated by $\triangle O P D / N=R \cdot S / N$ where $N$ is the number of fringes in the interference spectrum. The term $\Delta \psi$ in R.H.S. of Eq. 3.44 can be treated as the phase error caused by the finite sampling rate. It is then straightforward to obtain the initial phase shift $\Delta \varphi_{0}$ due to finite sampling rate using Eq. 4.2, where $k_{0}$ is the central wavenumber.

$$
\Delta \psi=\Delta \varphi_{0} \approx \frac{R \cdot S \cdot k_{0}^{2}}{\Delta k}
$$




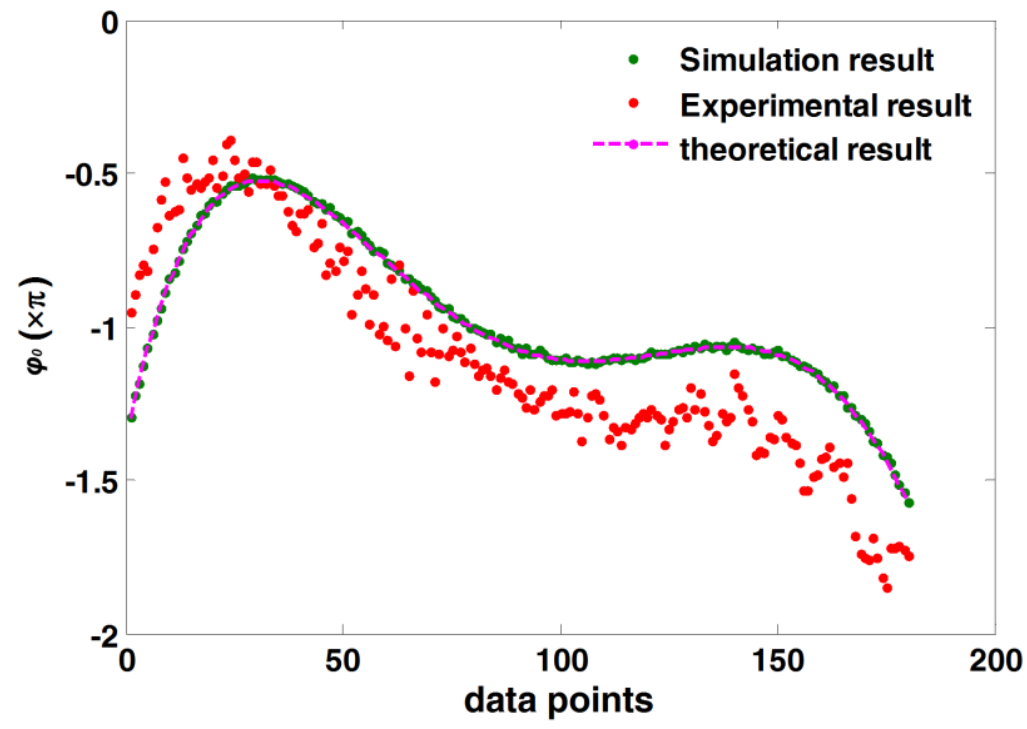

Figure 4-17. Comparison of simulated, experimental and theoretical variations in initial phase $\varphi 0(\tau)$ due to finite wavelength scan rate $S$.

Similar to the case for multimode-induced phase shifts, simulations in Section V show that finite-rate sampling can produce an unwanted phase shift of $\pi$ or greater.

In order to demonstrate the effects of finite sampling rate on initial phase, an IFPI sensor was heated from room temperature to $600^{\circ} \mathrm{C}$ in 30 minutes, while a total of 180 spectra were recorded at equal time intervals. The rate of temperature increase varied throughout the heating process, which led to a non-constant OPD changing rate $R$. The measured OPD and additional phase $\varphi_{\text {omeas }}$ of the sensor were estimated at each sampling point using the algorithm developed in Section IV. Next, the rate of OPD change R was estimated by applying a fifth-order polynomial fit to the measured OPD vs. sample number data.

Using Eq. 4.1, the estimated $R$ curve was applied to generate a set of simulated spectra containing the theoretical dependence of $\Phi(k)$ on sampling rate $S$ and OPD change rate $R$. This simulated data was then re-processed to calculate its additional phase $\varphi_{0 s i m}$. Figure 4-18 shows the comparison between measured additional phase $\varphi_{\text {omeas }}$, simulated additional phase $\varphi_{0 \text { sim }}$, and the theoretical sampling-induced phase shift in Eq. 4.2. All three curves clearly have the same shape, confirming the analytical sampling-induced phase shift result. A total initial phase shift of 
slightly less than $\pi$ is shown to result from this finite sampling rate phenomenon. As in Section V.A, the varying total phase algorithm is able to interrogate the sensor without introducing jumps in the demodulated output.

It should be noted that the two choices for sampling rates provided by CTS poses significant design considerations. As expressed in Eq. 4.2, a superficial additional phase term will be resulted if the sampling rate is finite, and the phase is inversely proportional to the rate. Also depicted from Eq. 4.2 is the fact that the additional phase is proportional to the OPD changing speed, which implies an increase in the phase if sampling frequency is fixed. For applications in which temperature changes drastically and fast, the additional phase will be large. This would potentially lead to demodulation jumps if the phase evolves over $\pi$. A solution, explicit from Eq. 4.2 , is to increase the sampling rate to compensate for the fast OPD changing. In other words, in such circumstances, $5 \mathrm{~Hz}$ scanning rate is preferred. A major drawback for faster scanning rate lies in the signal distortion associated with increased scanning speed. The spectrum for an ideal low-finesse IFPI sensor is purely sinusoidal, which is represented by a sharp harmonic peak in the Fourier transform domain without higher-order harmonics. However, as the scanning speed increases, the originally sinusoidal wave-pattern becomes distorted while still remains periodic. This phenomenon manifests clearly as higher order harmonics in the Fourier transform domain, which is undesirable if multiplexing is required. The harmonics may overlap with the fundamental frequency belonging to a certain multiplexed sensor and their interference will eventually generates significant demodulation errors. As a result, for real practice, if the temperature changes slowly, $0.5 \mathrm{~Hz}$ scanning rate is preferred in the sense that it guarantees minimum signal distortion and best demodulation accuracy.

\section{b. The algorithm for OPD demodulation}

The algorithm used for demodulation of the sensor OPD is based on the principle discussed in Section 3.3. It is important to note that the additional phase, together with OPD, is estimated. The benefit of using total phase demodulation has been given in Section 3.3, which suggests for better resolution and accuracy the estimated additional phase and OPD should be used together and plug into Eq. 3.50 for the total phase calculation. Eqs. 3.47 3.49 are used for the determination of OPD and the additional phase. 
The spectrometer provides equally spaced wavelength sampling, however the signal is sinusoidal with wavenumber. As a result, the digital spectrum, obtained at the pre-defined wavelength points, will have to be interpolated in order to have a transformed spectrum with evenly sampled wavenumber.
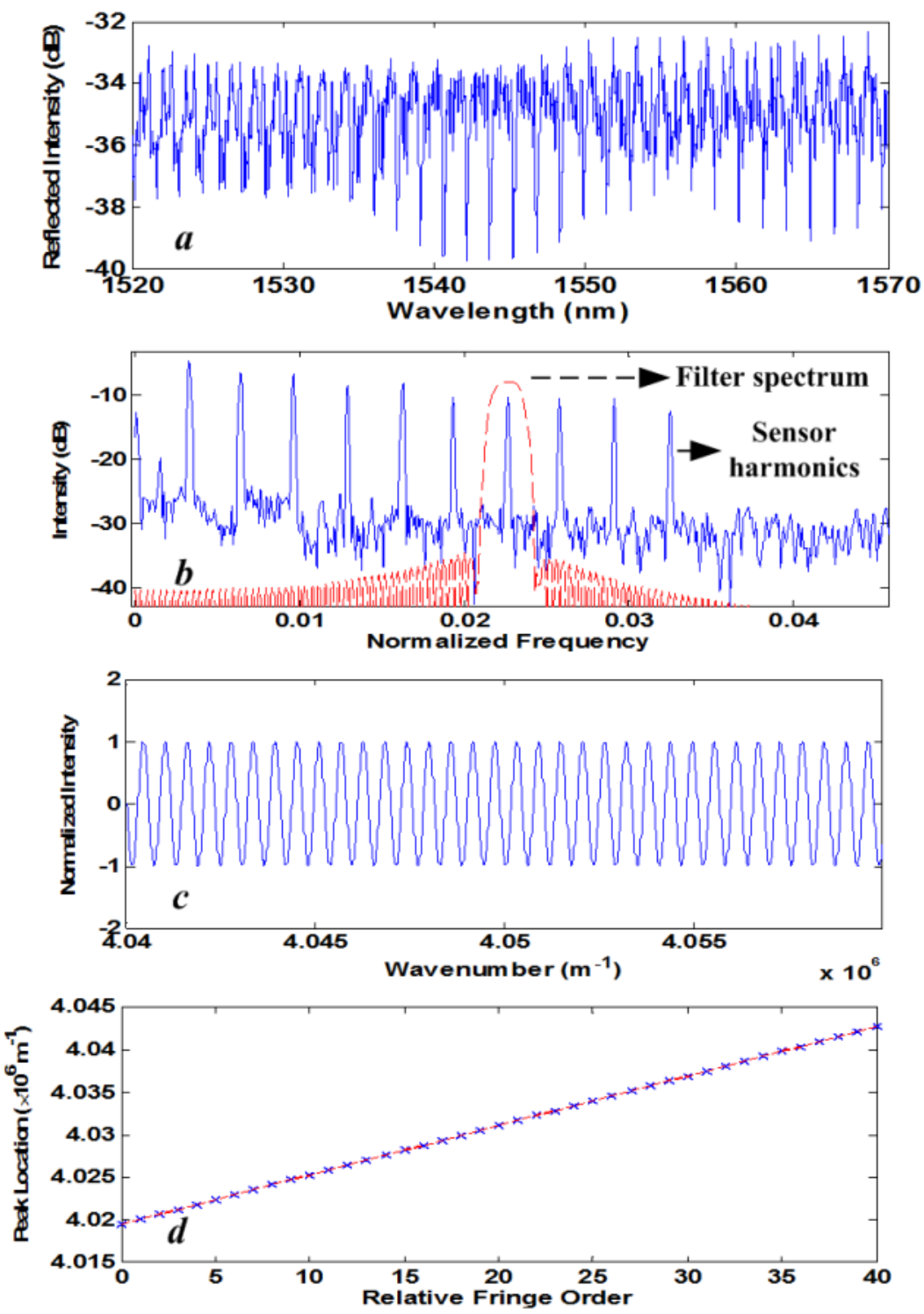

Figure 4-18. The signal processing approach shown in steps. (a) The original spectrum, with 10 sensors multiplexed. (b) The Fourier transform of the interpolated spectrum showing corresponding harmonics contributed by the sensors. The red dashed curve shows the digital bandpass filter applied to select one of the sensors. (c) The filtered signal, showing up as a pure sinusoid. (d) The tracked fringe peak locations plotted as a function of relative fringe order. Red solid line is the linear fitting. 
The interpolated signal is then converted with Fourier transform to the frequency domain, in which several distinct peaks show up, corresponding to the harmonics contributed to the multiplexed sensors. After determining the Fourier domain location of the peaks, a digital filter with order of 5000 is applied to select one particular sensor while the harmonics due to the other sensors are effectively suppressed. This is illustrated in Figure 418 (b).

The filtered signal appears as a pure sinusoid (Figure 418 (c)) with the frequency of the harmonic being the OPD of the selected sensor. Before demodulating its OPD, it is important to shift the spectrum back for 2500 points to compensate the delay caused by filtering, otherwise a significant additional phase estimation error will result.

With the treated harmonic signal, it is straightforward to calculate the fringe peak locations, and their relative fringe orders. Plotted in Figure 4.18 (d) in blue cross is the peak location versus fringe order calculated based on the spectrum shown in 4.18 (c). The locations are denoted as $k_{\mathrm{m}}$ and fringe orders are denoted as $m$, their values are subsequently applied in Eqs. 3.47 3.50 for the estimation of OPD and additional phase $\varphi_{0}$, and eventually the total phase.

The Matlab code for the program is provided in the appendix.

\subsubsection{Temperature calibration and measurement results}

Sensors were fabricated with cavity lengths controlled to be $N \times 520 \mu \mathrm{m}$, where $N$ is an integer ranging from 1 to 6 representing the sensor number, totally 6 sensors were multiplexed in a single link. $520 \mu \mathrm{m}$ corresponds to the refocusing pitch length (see Section 3.2) in a $62.5 \mu \mathrm{m}$ core multi-mode fiber (Thorlabs, GIF625). Lead-in fiber was standard single-mode fiber (Corning, SMF28). The sensor link was calibrated in a furnace at 40 discrete temperature points (with a K-type thermal couple) to $800{ }^{\circ} \mathrm{C}$. The temperature values were fitted to the corresponding OPD with a second order polynomial. The 2-sigma error of the fitting represents the accuracy (plus the inaccuracy caused by the thermal couple). Finally the resolution was obtained with the whole chain placed in a temperature-static environment and calculating the 2sigma of the output (twice the standard deviation). The temperature calibration curves are depicted in Figure 4-19. 

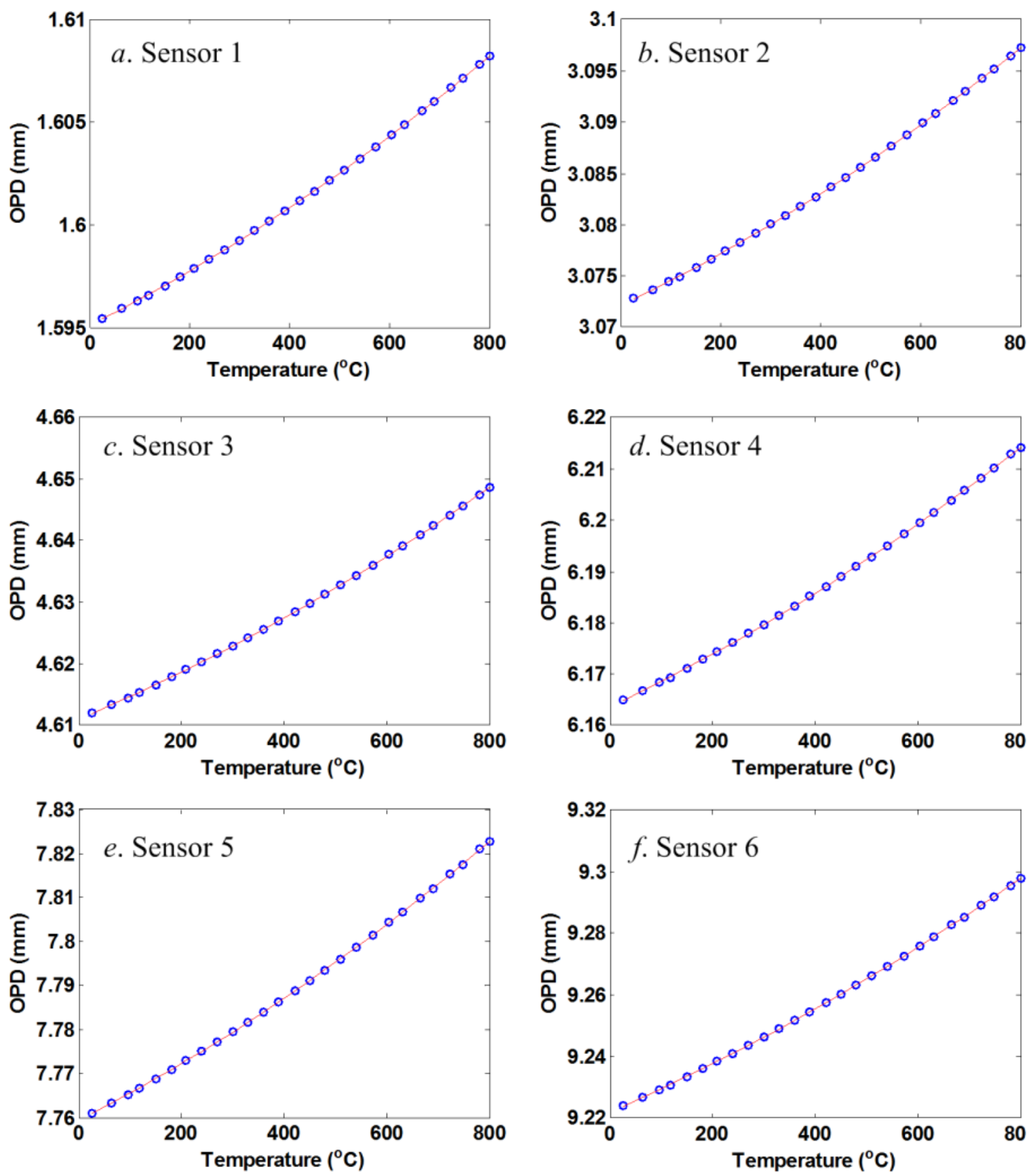

Figure 4-19 Temperature calibration curves of an IFPI link multiplexed with 6 sensors. 
The calibration curves are fitted with second-order polynomial as

$$
L=p_{2} \cdot T^{2}+p_{1} \cdot T+p_{0}
$$

where $T$ denotes temperature, and $L$ is the measured OPD. $p_{\mathrm{i}}(i=0,1,2)$ are the calibration coefficients determined with fitting. The values of the coefficients are summarized in Table 4.1Table 4.1. Temperature calibration coefficients.. The 2-sigma error of the sensor is estimated with the following equation

$$
\varepsilon=2 \operatorname{std}[L-\mathbf{P}(T)] / p_{1}
$$

In the equation $\operatorname{std}[\cdot]$ denotes standard deviation, $\mathbf{P}$ is the operation using the fitting polynomial (as in Eq. 4.3). The accuracy of the sensor is estimated by

$$
\sigma=\left[\varepsilon^{2}-\kappa^{2}\right]^{1 / 2}
$$

In which $\kappa$ is the error of the thermocouple, which is $2^{\circ} \mathrm{C}$. The values for $\varepsilon$ and $\sigma$ for all the sensors are listed in Table 4.2.

Table 4.1. Temperature calibration coefficients.

\begin{tabular}{c|ccc}
\hline \hline Sensor & $p_{2}\left(\times 10^{-11}\right)$ & $p_{1}\left(\times 10^{-8}\right)$ & $p_{0}\left(\times 10^{-3}\right)$ \\
\hline 1 & 0.5260875 & 1.218083 & 1.595098 \\
2 & 1.034270 & 2.305197 & 3.072154 \\
3 & 1.482535 & 3.518189 & 4.611009 \\
4 & 1.994117 & 4.722386 & 6.163666 \\
5 & 2.478893 & 5.936638 & 7.759351 \\
6 & 2.839841 & 7.210341 & 9.222042 \\
\hline
\end{tabular}

Table 4.2. Temperature measurement accuracy.

\begin{tabular}{c|cccccc}
\hline \hline Sensor & 1 & 2 & 3 & 4 & 5 & 6 \\
\hline$\varepsilon\left({ }^{\circ} \mathrm{C}\right)$ & 3.04 & 2.98 & 2.82 & 2.99 & 2.65 & 2.75 \\
$\sigma\left({ }^{\circ} \mathrm{C}\right)$ & 2.29 & 2.21 & 1.99 & 2.22 & 1.74 & 1.84 \\
\hline
\end{tabular}


The temperature measurement resolution was estimated by stabilizing the environmental temperature and taking 700 data. The data obtained shows Gaussian distribution with its 2-sigma (twice the standard deviation of the normal distribution) being defined as the resolution. Histogram was used to visualize the noise distribution, and the calculated histogram for sensor 1 , together with its Gaussian fitting and the normal distribution was plotted in Figure 4-20.

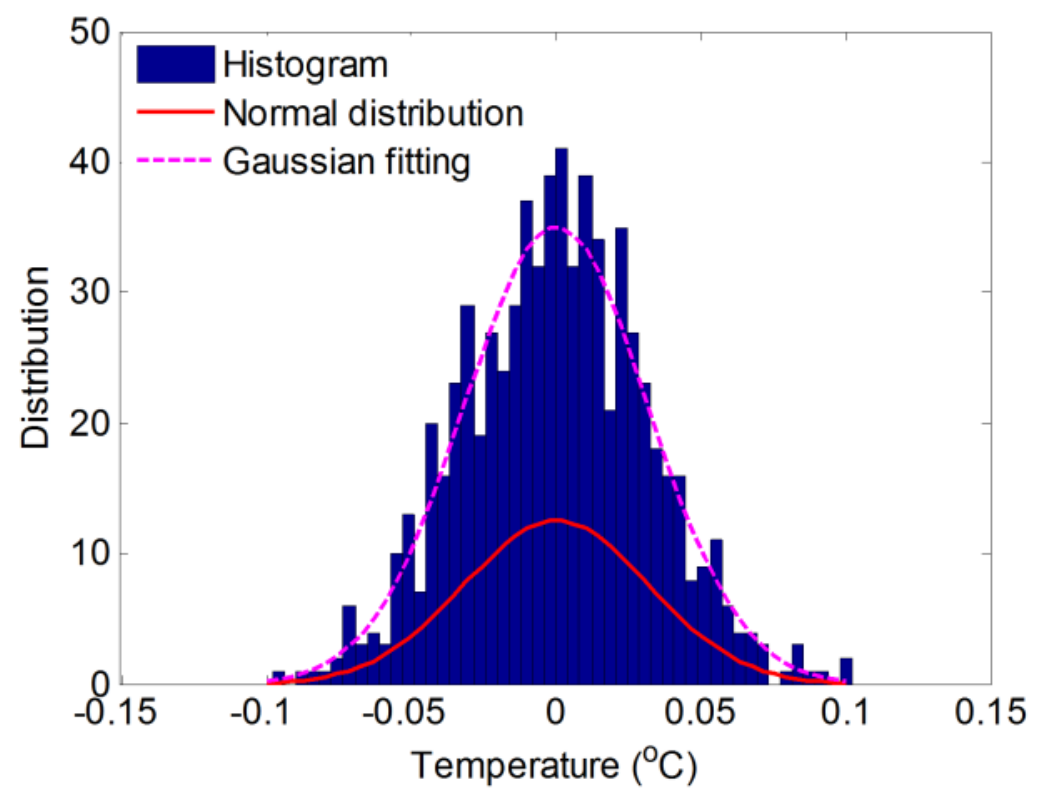

Figure 4-20. Calculated histogram and corresponding distribution for sensor 1.

In the same manner, the resolutions for the other five sensors were obtained and summarized in Table 4.3.

Table 4.3. Measured temperature resolution of the sensors.

\begin{tabular}{c|cccccc}
\hline \hline Sensor & 1 & 2 & 3 & 4 & 5 & 6 \\
\hline$\delta\left({ }^{\circ} \mathrm{C}\right)$ & 0.064 & 0.060 & 0.049 & 0.048 & 0.048 & 0.048 \\
\hline
\end{tabular}

\subsubsection{Strain calibration and measurement results}

a. Strain calibration and characterization

Denoting the strain, defined as $\mathrm{d} L / L$ (where $L$ is the physical cavity length) to be $\varepsilon$, the relative OPD change as a function of the applied strain can be found by 


$$
\frac{d \mathrm{OPD}}{\mathrm{OPD}}=\left[1-P_{e}(T)\right] \varepsilon
$$

in which $P_{\mathrm{e}}(T)$ is the strain-optic coefficient which represents the refractive index change associated with applied strain, and the argument $T$ denotes the fact that this coefficient is dependent on temperature. In order to measure $P_{\mathrm{e}}$ and its temperature dependence, we calibrated a strain sensor with different strain loading and environmental temperatures. The calibration curves are shown in Figure 4-21.

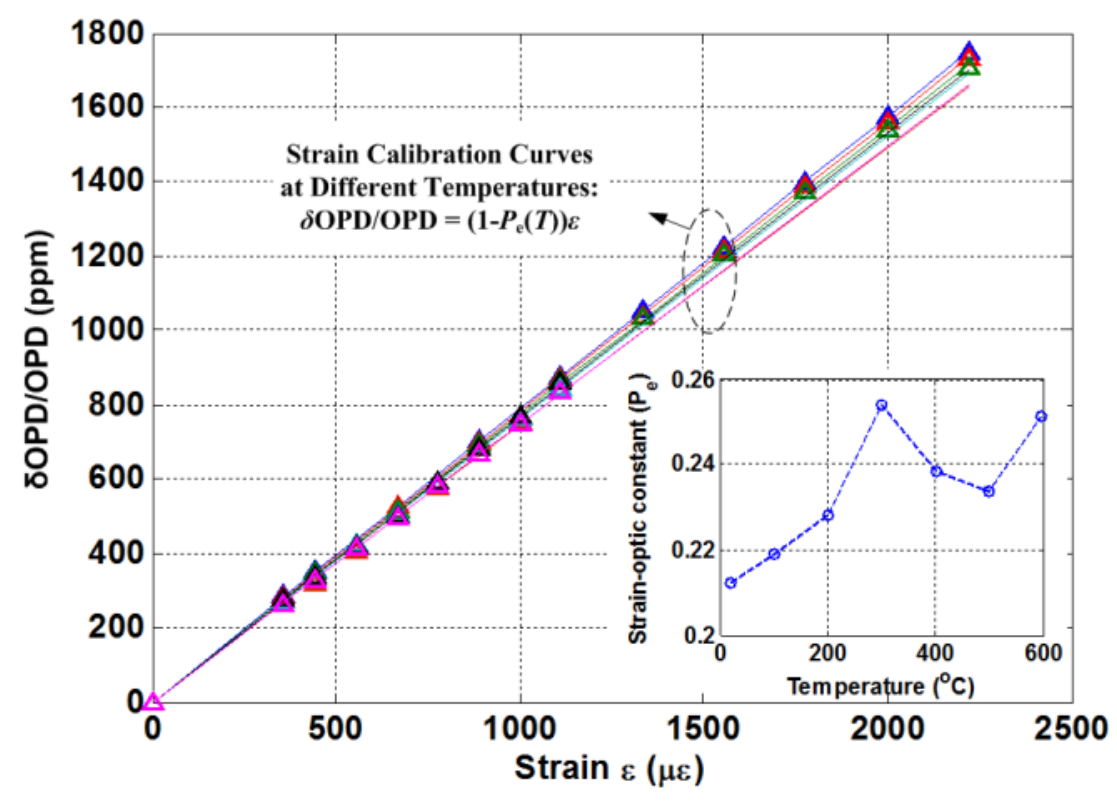

Figure 4-21. Strain calibration at different temperatures. Different slopes correspond to different strainoptic coefficient at various temperatures. The inset shows the calculated strain-optic coefficient at different temperatures.

The slope of the strain-OPD calibration curve varies with temperature, using Eq. 4.6, the corresponding strain-optic coefficients at a number of temperature points were calculated and displayed in the inset figure. From the data, we concluded that the strain-optic coefficient has the following temperature dependence:

$$
P_{e}=A(T-C)+B
$$



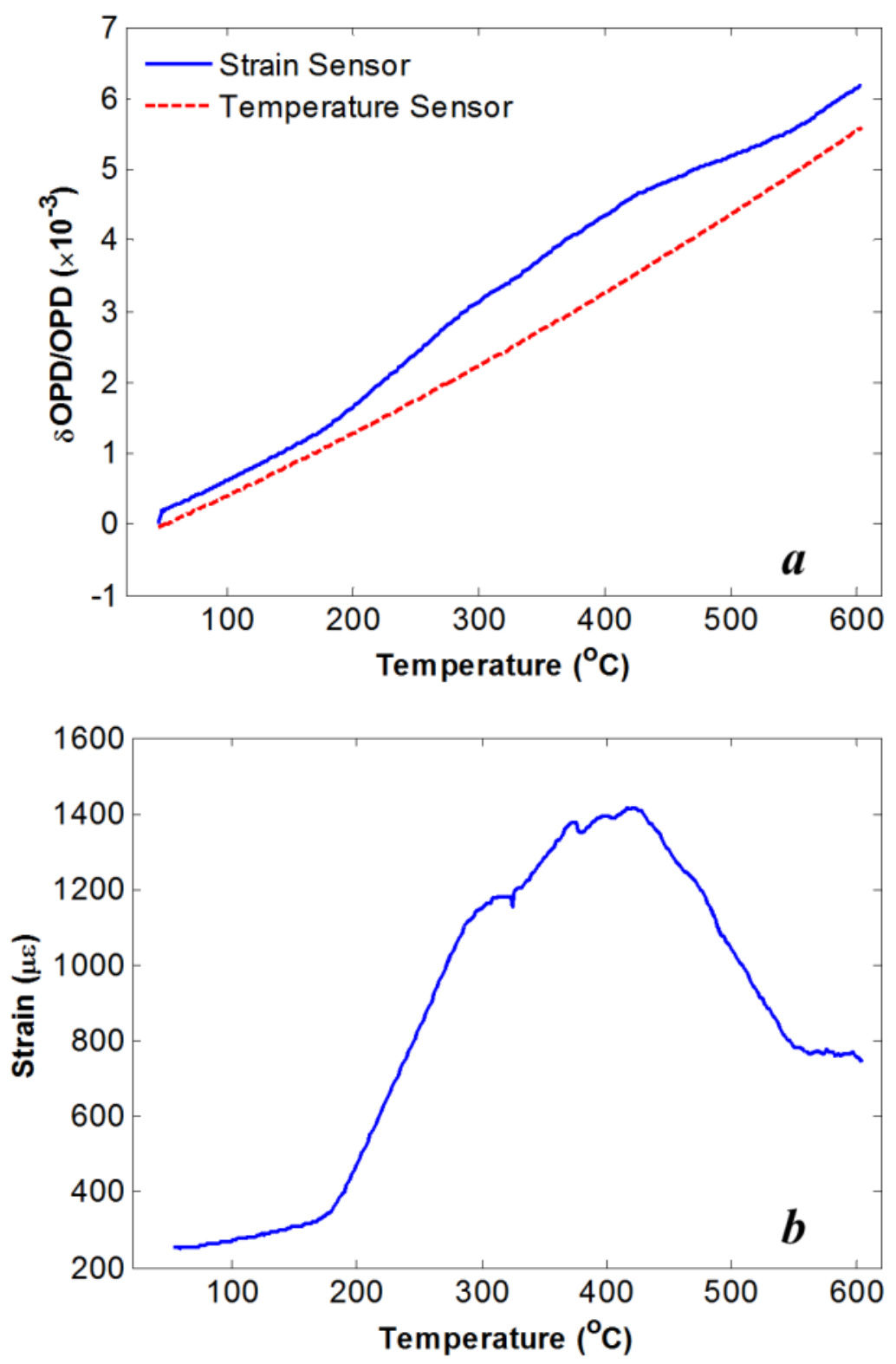

Figure 4-22. Strain measurement with temperature compensation. (a) The OPD change measured with a strain sensor and a co-located temperature sensor as a function of temperature. (b) The calculated strain during the measurement process.

where $A=6.957 \times 10^{-5}\left(/^{\circ} \mathrm{C}\right), B=0.21$, and $C=30^{\circ} \mathrm{C}$. With similar definitions used for temperature measurement, the strain measurement accuracy and resolution was found to be 3.79 $\mu \varepsilon$ and $2.96 \mu \varepsilon$, respectively. 


\section{b. Strain measurement}

Denoting the temperature calibration relationship as $\mathbf{P}(T)$, where $T$ is the temperature measured with a co-located IFPI temperature sensor, the strain applied on the strain sensor was calculated as:

$$
\varepsilon=[1-\mathbf{P}(T) / \mathrm{OPD}] /\left(1-P_{e}(T)\right)
$$

In this equation the only variable is the OPD which is being measured with the strain sensor, all other parameters are constants and have been defined earlier. During the temperature increase cycle, the $\delta \mathrm{OPD} / \mathrm{OPD}$ values for the strain sensor and the co-located temperature sensor were calculated and depicted in Figure 4-22 (a), the calculated strain during the temperature cycle was shown in Figure 4-22 (b). 


\section{SENSOR FIELD TEST}

On November $16^{\text {th }}$, 2011, a field test using the IFPI sensor for distributed temperature measurement was performed at the National Energy Technology Laboratory (NETL) at the US Department of Energy (DOE) at Morgantown, West Virginia. This section reviewed the preparation and results of the field test.

Due to their compact size, sensitivity, and ability to be multiplexed, IFPI sensors are excellent candidates for almost any multipoint temperature or strain application. Installing a sensor chain on an object as small as a 2"x2" coupon presents a unique set of challenges: fiber bending loss, precise fabrication techniques, and fragility issues.

It is known that using an SMF28 lead-in fiber, a multimode fiber section as a Fabry-Perot cavity, and an additional SMF28 fiber as the tail results in a structure that generates strong interference fringes while remaining robust. However, due to geometric constraints resulting from the size of the test coupon and sensor placement requirements, fabricating a sensor chain with appropriate sensor spacing and excellent temperature response characteristics proved a significant challenge. Issues addressed include inter-sensor interference, high-temperature mechanical reinforcement for bare fiber sections, and high bending losses.

\subsection{Background introduction}

Optical fiber sensors are recognized for their resistance to corrosion and ability to withstand all but the most extreme temperatures. These characteristics make them a valuable asset in monitoring harsh environments. While these sensors have been adopted for a variety of industrial applications, due to the fact that they are glass fibers, they inherently suffer from issues with mechanical robustness, and extreme bends.

The small-scale combustion chamber at the National Energy Technology Laboratory is designed to simulate the operating conditions found in gas turbines. Adjusting environmental variables and measuring their effect on temperature, burn efficiency, and chemical byproducts is the primary focus of this rig. As these complex processes are optimized, the improvements are incorporated into industrial power generation units, resulting in higher efficiency, lower 
emissions, and in the end, lower operating costs. One variable that significantly affects combustion is the flow of cooling air downstream from the flame. One approach to understanding the impact of flow direction and magnitude on the overall process is measuring the temperature gradients across the coupon the air flows through as it is injected into the system. The coupon measures 2" x 2" and is secured at the bottom of a tube, as shown at the very lefthand side of Figure 5-1.

It was determined that the temperate should be measured at four distinct locations on this

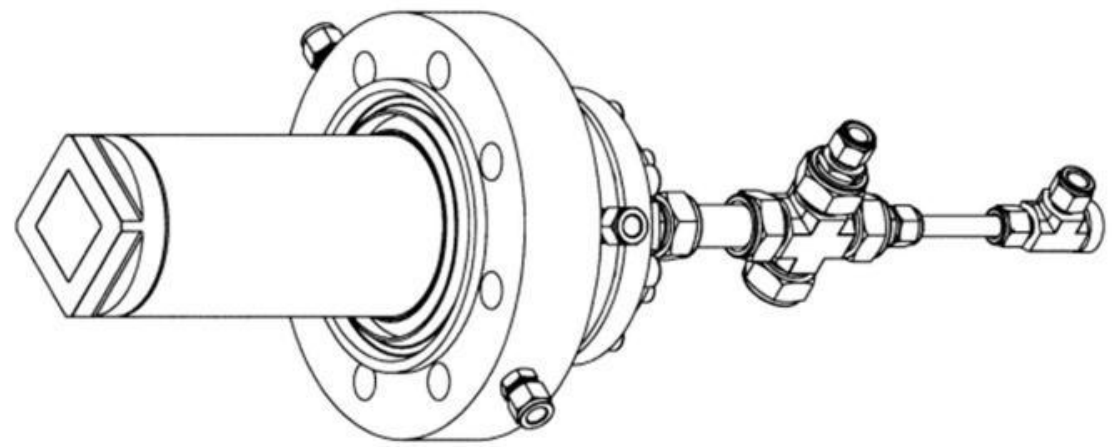

Figure 5-1. Drawing of he cooling air injection setup

coupon while pressurized high-velocity cooling air flowed through the coupon and the combustion chamber was subjected to a battery of tests. In many ways this provided an ideal test bed for addressing the above-mentioned weaknesses associated with optical fibers: the highvelocity air challenged the mechanical robustness and the coupon dimensions inherently required sharp bending.

\subsection{Sensor chain design}

Finalizing the sensor spacing and chain routing was one of the major challenges associated with this project. The baseline coupon geometry is shown in Figure 5-2. The initial plan was to space each sensor approximately $3.2 \mathrm{~cm}$ apart from each other in a simple spiral shape, with the fiber surrounded by a stainless steel sheath and held to the surface of the coupon by four welded tubes. In order to fabricate the sensors, a section of bare fiber is necessary, and the original concept was to recoat the entire chain with a high-temperature polyimide coating and then insert this fiber into the stainless capillary tube before routing it through the hold-down tubes. 
This approach failed for numerous reasons. First and foremost, the sensors were located too closely together, which seriously affected the signal quality. Additionally, the stainless steel tubing was extremely abrasive on the fiber, and when this was combined with a less-than-ideal polyimide coating, repeated breakages were the result. We have carried out extensive studies of recoating the fiber for mechanical enforcement, as will be discussed below.

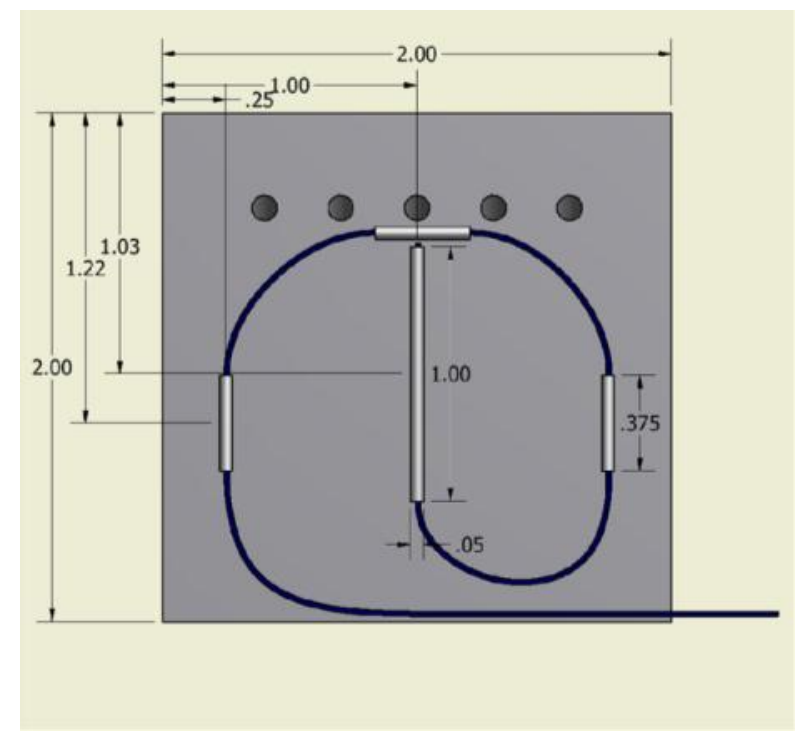

Figure 5-2. Base coupon geometry with dimensions.

\section{a. Fiber recoating}

The fiber recoating setup is shown in Figure 5-3(a), which comprises three parts: a glass chamber with the polyimide solution, a tube furnace (for wet polymer drying), and a thin glass tube for coating reshaping (to keep coating uniform). The three pieces are aligned vertically. The longterm bending performance of the polyimide-coated fibers was tested at $350^{\circ} \mathrm{C}$. Due to the effort required to fabricate a single test piece, a large sample size was not feasible, but 15 coated fibers were fixed at a $1.9 \mathrm{~cm}$ bend radius - the minimum bend radius experienced by a fiber when installed on the coupon - and placed into a furnace. The survival rate after one week was over $80 \%$. The performance of the coating can be further improved by increasing the thickness and uniformity of the coating, and a pursuing of achieving optimized performance is underway. 

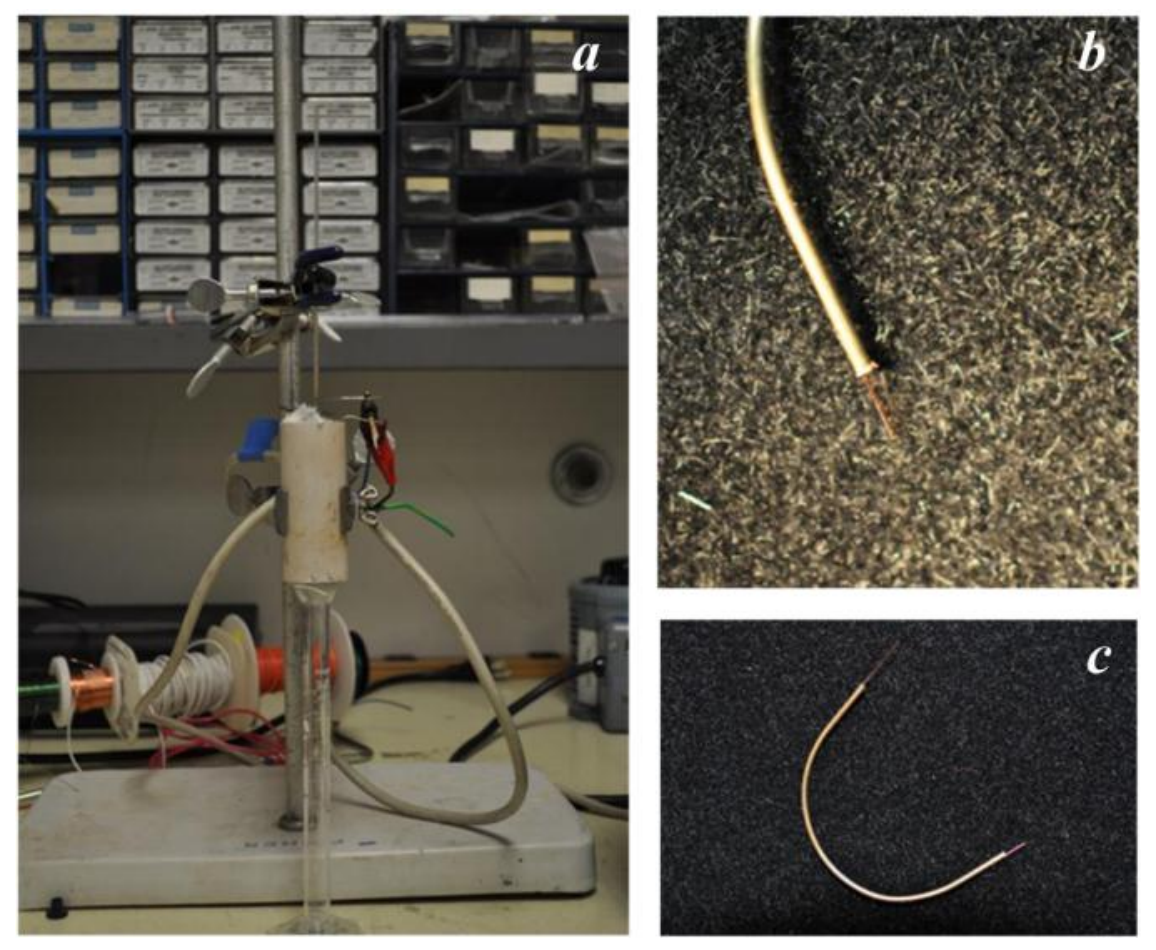

Figure 5-3. Fiber coating apparatus and the testing of the bending of the coated fiber. (a) Fiber coating setup: a tube furnace is aligned with a silica tubing in vertical position. On the bottom of the heating furnace is a glass chamber which contains the polyimide solution. The dip-coating process will be performed by firstly dipping the bare fiber inside the solution, and gradually pull it up through the furnace and the reshaping glass tube. (b) and (c): bending test of the coated fiber. The yellow thin piece in the white metal tubing is the coated fiber, and the whole piece was curled to a radius of 0.75 ”, and heated. (b) is a zoomed-in view of $(c)$.

The fiber coating process was much trickier than anticipated. The primary issue was coating uniformity. The polyimide tended to coagulate rather than adhere uniformly to the fiber, which resulted in areas of nonuniform thickness that acted as stress concentrators. When the fiber was bent sharply the non-uniform locations would inevitably snap. It was also postulated that microcracks in the glass due to the decoating process could be responsible for the breakage, but breakages occurred even when acetone was used to remove the polymer coating from the fiber.

Significant effort was put into developing a more sophisticated coating process, but nonuniformities still cropped up occasionally, as shown in Figure 5-4. These nonuniformities compromised the repeatability of the work. When this issue was coupled with an update that temperatures in the combustion chamber were higher than initially anticipated, the fiber 
recoating process was dropped entirely in favor of gold-coated fiber. A summary of the materials used and resultant coating quality is found in Table 5.1. With additional work to eliminate nonuniformities, the PI-2525 from HD Microsystems should be an excellent fiber coating for applications requiring temperatures up to $400^{\circ} \mathrm{C}$.

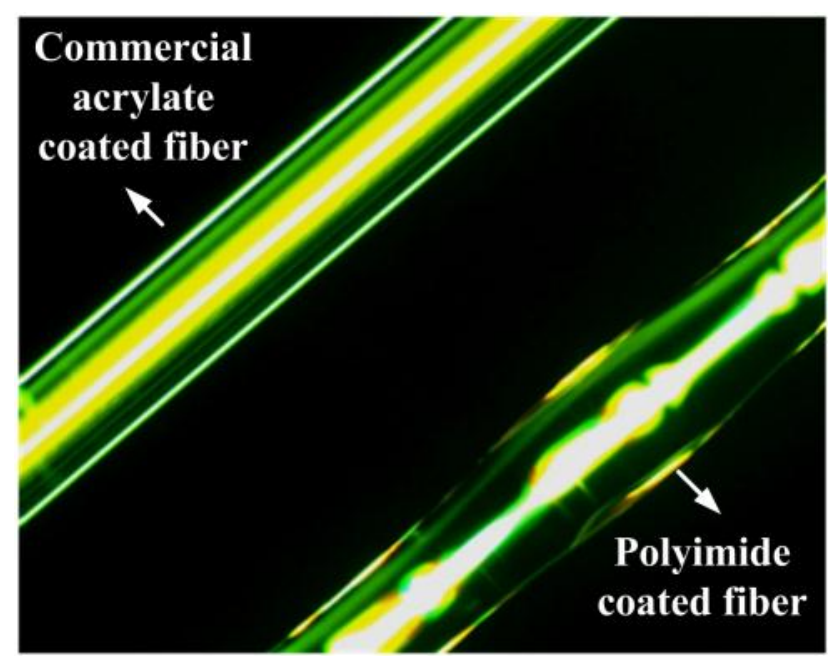

Figure 5-4. Nonuniform polyimide coating (lower-right) compared to commercial acylate coated fiber (upper-left).

Table 5.1. Materials and coating quality.

\begin{tabular}{c|c|c|c}
\hline \hline Product & MFG & Type & Results \\
\hline HP1632 & Michelman & Water-based polyimide & $\begin{array}{c}\text { Viscosity very low, requiring } 30 \\
\text { layers. Coating is brittle brittle. }\end{array}$ \\
\hline HP1432 & Michelman & Water-based polyimide & $\begin{array}{c}\text { Higher viscosity, but coating remains } \\
\text { brittle }\end{array}$ \\
\hline PA845 & Michelman & Water-based nylon solution & $\begin{array}{c}\text { Borderline temperature performance. } \\
\text { Good mechanical reinforcement }\end{array}$ \\
\hline PI-2525 & Polyazeridine & $\begin{array}{c}\text { Cross-linker for improving } \\
\text { flexibility of Michelman }\end{array}$ & $\begin{array}{c}\text { No obvious results. } \\
\text { Microsystems }\end{array}$ \\
\hline
\end{tabular}




\section{b. Gold-coated fiber}

After dropping the initially proposed configuration, all subsequent designs relied upon goldcoated fiber with short sections of bare fiber corresponding to each sensor. These bare fiber sections were protected by sections of commercial polyimide-coated glass capillary tubing. This approach eliminated the issues associated with recoating and the stainless steel sheath, but the problem of determining the appropriate inter-sensor spacing remained, as well as the challenge of securing the extensive amount of additional fiber to the coupon in such a limited space. After some analysis and experimentation, it was determined that spacing the sensors approximately $15 \mathrm{~cm}$ apart provided a the best signal quality by balancing the inter-sensor noise issue associated with short spacing with the increased bending losses associated with longer distances between sensors.

This balance proved very successful from a signal quality standpoint, and the additional fiber was initially just routed through the original hold-down tubing. However, during simulated environmental testing, it was found that the fibers rubbed against the edge of the hold-down tubes and breakages resulted. As a result, the free-form routing approach was adopted, and this proved very successful both in the lab and the field-test environment. All of these approaches are summarized with relevant photos in table 2 found on the next page.

\section{c. Sensor chain configuration}

We have adopted four sensor chain configurations throughout the project. We started with the configuration shown in the inset figure I of Table 5.2, in which a section of stainless-steel tubing with matched outer diameter with the inner diameter of the hold-down tubes was used to secure the sensing fiber. We originally designed this schematic expecting the best mechanical integrity provided by the metal tubing. However, as we demonstrated through designed experiments, the fiber zig-zaged inside the metal tubing to introduce highly localized strain, which consequently break the fiber during installation. Even with the strong gold-coated fiber, breakage during installation was not avoidable, as a result, this scheme was finally abandoned. Later we switched to the simple mounting scheme using gold coated fiber, shown in the inset figure 1 of Table 5.2. Such geometry provides great flexibility during installation, and demonstrated great mechanical endurance. However, a major issue was that the sensors have to be cascaded with very small spacing $(\sim 1 ")$ which generates significant cross-talk between 
adjacent sensors. To eliminate the cross-talk, we developed the "multi-wrap" scheme shown in figure 2 in Table 5.2. This scheme demonstrated the best performances in terms of mechanical strength and signal quality by then, but the sharp bending between the neighboring hold down tubes initiates the potential of fiber breakage at high temperature. We finally adopted the mounting scheme shown in figure 3 of Table 5.2. This scheme provides the best flexibility in sensor installation and robustness by eliminating sharp bending unavoidable in any of the former schemes.

Table 5.2. Summary of investigated coupon configurations.

\begin{tabular}{|c|c|c|}
\hline $\begin{array}{l}\text { Scheme } \\
\text { number }\end{array}$ & Characteristics & Figure \\
\hline I & $\begin{array}{l}\text { Initially Proposed Configuration } \\
\text { Benefits: } \\
\text { Elegant; Stainless protective tubing is very robust; Good } \\
\text { thermal transfer } \\
\text { Issues: } \\
\text { Interference between closely spaced sensors; } \\
\text { Stainless tubing abrades fiber, resulting in breakagesIssue with } \\
\text { antireflective end being too close to steel tubing. }\end{array}$ & 000 \\
\hline 1 & $\begin{array}{l}\text { Glass Capillary Tubes over Bare-Fiber Sections } \\
\text { Benefits: } \\
\text { Installation is tricky but repeatable; } \\
\text { Issues: } \\
\text { Interference between closely spaced sensors; } \\
\text { Sensor response is reduced due to insulating glass tubing; } \\
\text { Issue with antireflective end being too close to steel tubing. }\end{array}$ & \\
\hline 2 & $\begin{array}{l}\text { Increased Inter-sensor Spacing } \\
\text { Benefits: } \\
\text { Installation is simple; } \\
\text { Long spacing resolves sensor crosstalk issue } \\
\text { Issues: } \\
\text { Higher bending losses due to the number of tight bends; } \\
\text { Loops are difficult to control and are easily tangled. }\end{array}$ & \\
\hline 3 & $\begin{array}{l}\text { Free-form Routing } \\
\text { Benefits: } \\
\text { Minimizes regions of stress concentration near the edges of } \\
\text { hold-down tubes, breakages much less frequent; } \\
\text { Bending losses are reduced; less crosstalk. } \\
\text { Issues: } \\
\text { Complicating placement into test rig. }\end{array}$ & \\
\hline
\end{tabular}




\subsection{Sensor fabrication}

a. Sensor fabrication

The major steps for the sensor fabrication using Au-coated fiber are summarized in Table 5.3. The key component for the fabrication is a precision cleaver with a 1D translation stage. The stage and cleaver have their fiber axis aligned and their positions fixed. During fabrication, the IFPI cavity length can be easily controlled by adjusting the 1D stage after accurate alignment of the splicing point with respect to the cleaver's blade.

Table 5.3. Sensor fabrication procedure.

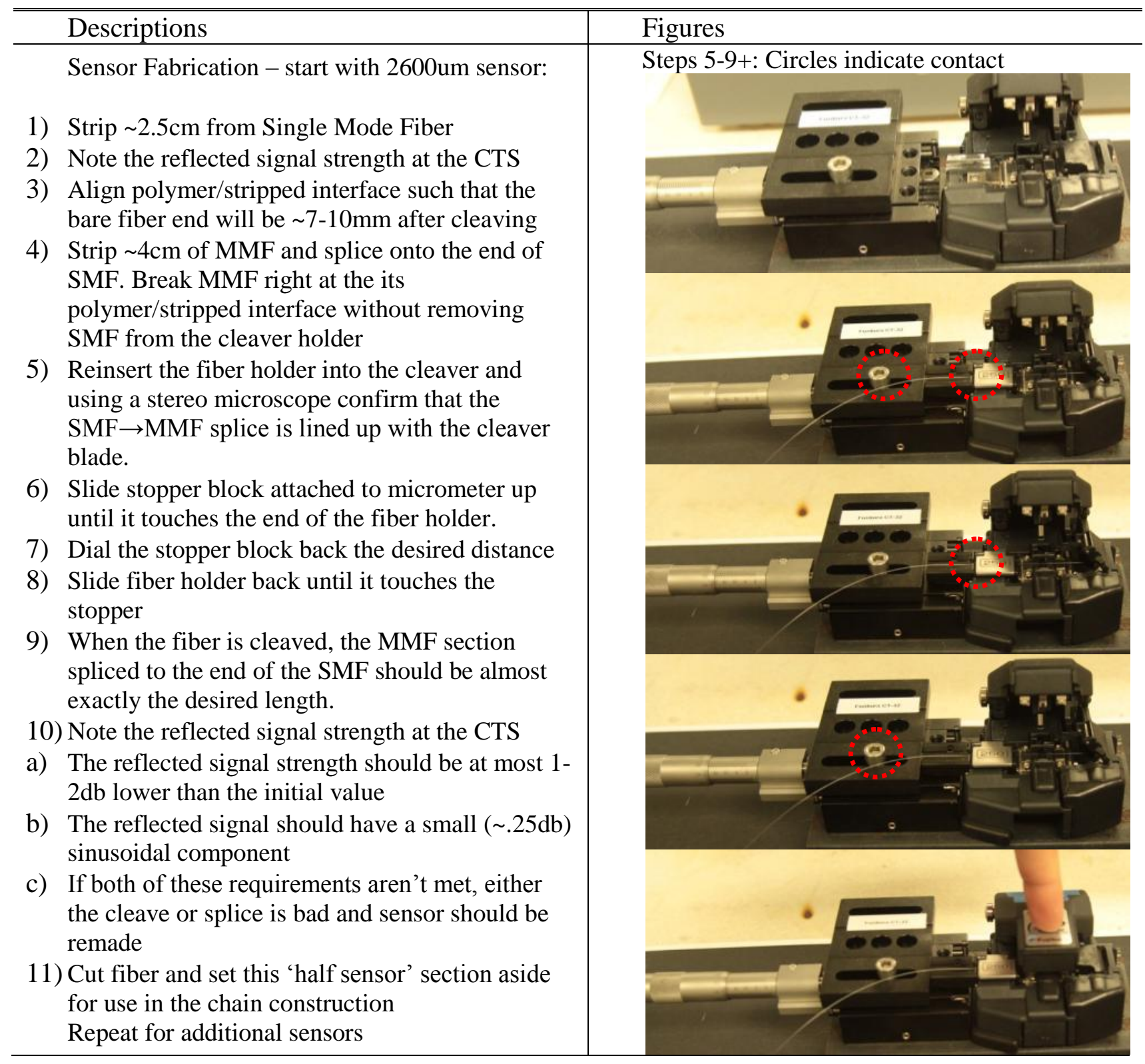


b. Chain fabrication

The chain fabrication process is summarized below (and illustrated in Figure 5-5):

1. With a half-sensor still attached to the $S M F$, strip $\sim 2.5 \mathrm{~cm}$ from the end of the next sensor to be located in the chain, cleave the end in such a way that there is a minimal amount of bare fiber remaining (same as step \#1 above).

2. Splice the SMF portion of the half-sensor link onto the end of the MMF section of the halfsensor still attached to the CTS. This completes the first full sensor. Loop a small knot into the middle of the second half-sensor link to eliminate any reflection from the end of the fiber, and then capture the spectrum from the CTS. This spectrum represents the performance of the first sensor in the link

3. Repeat this process for the remaining half-sensor links

4. Fabricate an antireflective end by either 'junk cleaving' an SMF fiber in such a way that $60 \mathrm{~dB}$ of loss is induced, or polish the end face at a $12^{\circ}$ angle for antireflection purposes while monitoring the loss

5. Splice this antireflective section onto the end of the chain.

6. Capture and characterize performance.

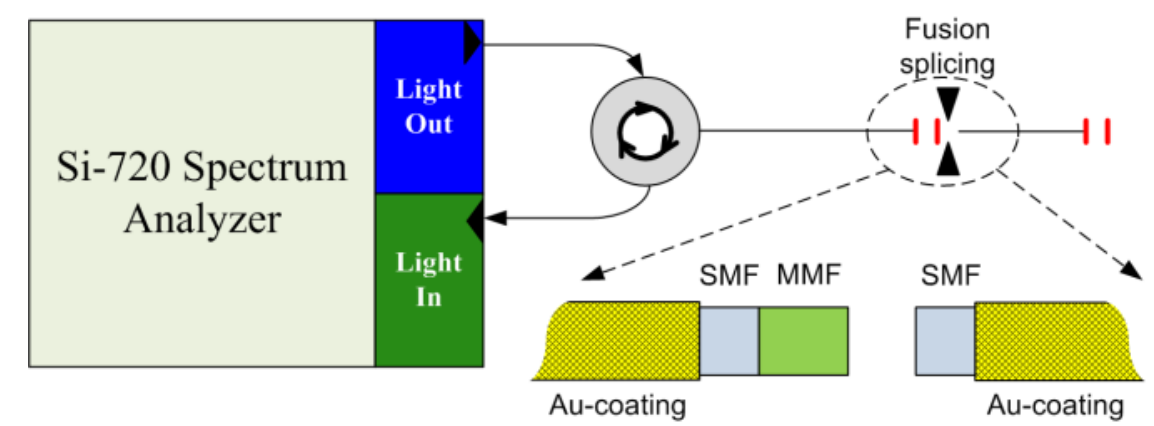

Figure 5-5. Illustration of the sensor chain fabrication process. SMF: single-mode fiber; MMF: multi-mode fiber.

\subsection{Sensor performance characterization}

\section{a. Sensor evaluation}

Irrespective of the fiber used or inter-sensor spacing, the chains were characterized using the same technique and then compared with one another. Due to improvements in technique and 
process optimization, total time for fabricating a viable sensor chain was controlled to be within 2 hours. The spectrum of the fabricated chain was captured by a CTS. Both the wavelength domain and the wavenumber domain provide valuable insight into the performance of a given chain. Figure 5-6 shows the characteristics of a typical chain.

The peak-to-valley strength as well as sharpness of the spikes in the bottom two plots have a direct impact on the accuracy and demodulability of the sensors. Each spike represents a single sensor. Both plots represent the same chain, but the signal strength for sensors 2, 3, and 4 clearly falls off after installation due to bending losses.

The upper two plots clearly show the roughly periodic nature of the spectrum, which is a superposition of frequencies corresponding to each of the sensors combined with any background noise. The 'purity' of each period in the sinusoid associated with each sensor can be quantified. The 'chirp', or deviation from a pure central frequency, is shown for each sensor in Figure 5-7, and has strong correlation with overall accuracy and sensitivity.
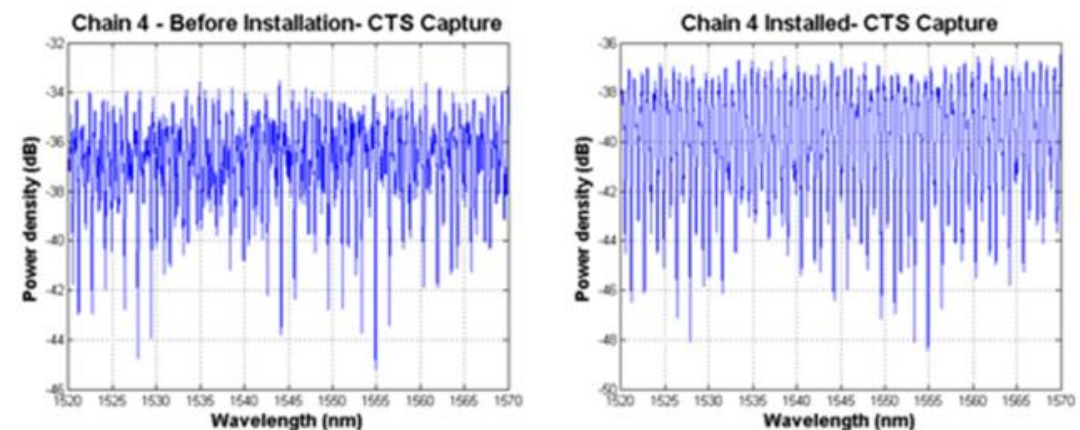

$a$

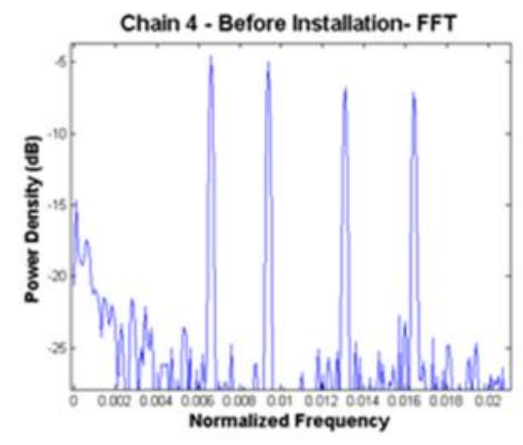

b

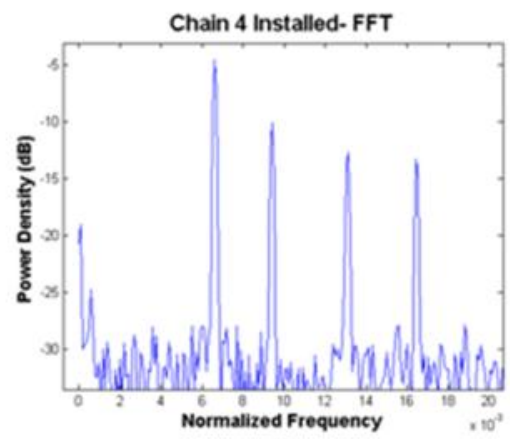

$c$

$d$

Figure 5-6. Chain characterization results. (a) and (b) show the spectrum obtained by CTS. (c) and (d) show the Fourier Transform of the spectra in (a) and (b). The figures represent a change in down-link signal quality after chain installation, as reflected by the decreased harmonic intensities. 
We found that the frequency impurity, as denoted by "chirp" of the spectrum, was an effective measure of the sensor's performance. Larger frequency chirp amounts to greater demodulation uncertainties, in turn, the sensor accuracy would sacrifice. Through extended experiments, we qualitatively confirmed the relationship between chirp intensity and sensor accuracy. Which is the bases for using the chirp intensity as an evaluation standards upon which different sensor links were compared. It was discovered that the chirp was highly dependent on several factors, the most prominent among them being the sensor spacing. Experimental evidences positively supported a significant increasing tendency of the chirp with decreased sensor spacing. This was attributed to the crosstalk between neighboring sensors caused by nonvanishing cladding mode. When spacing is large, the excited cladding modes at the fiber splicing interface will experience significant attenuation when reach the successive sensors, however the attenuation decreases when spacing between sensors is reduced.
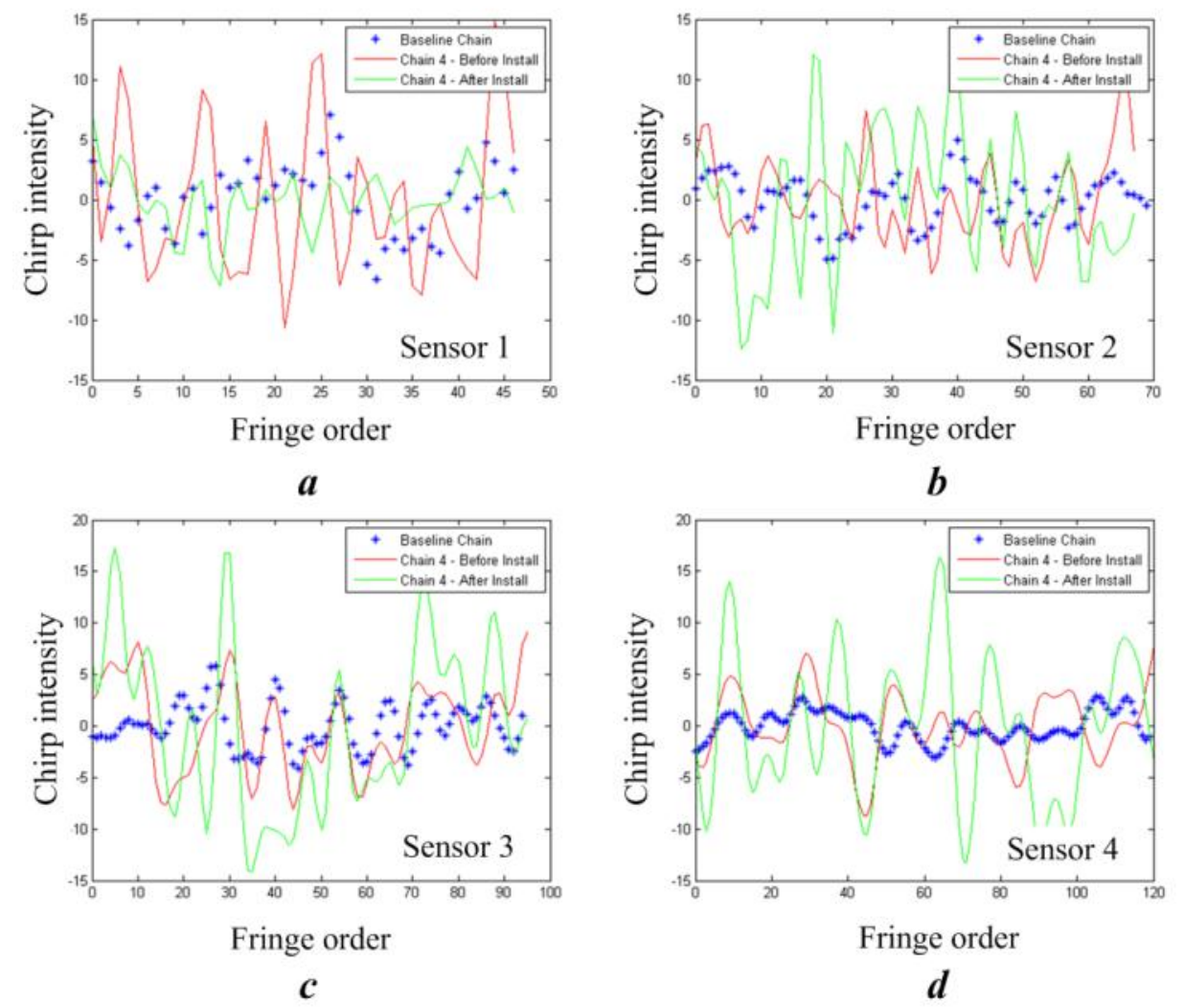

Figure 5-7. Calculated chirp intensities of the sensors multiplexed in one link. The cavity lengths are (a) $1040 \mu \mathrm{m}$, (b) $1560 \mu \mathrm{m}$, (c) $2080 \mu \mathrm{m}$ and (d) $2600 \mu \mathrm{m}$. Colors represent different installation steps. 
One important detail to note when analyzing these results is that the exact correlation between the chirp and sensor accuracy and sensitivity is unknown. As such, each sensor's performance must be compared to another sensor with proven performance. In Figure 5-7, the installed chain shows a significantly increased chirp as compared to that before installation. This is attributed to two factors. 1) the loss associated with bending decreases the signal to noise ratio, which correspondingly increases the effective power of noise falling within the digital bandpass filter; 2) the bending of the fiber induces stronger cladding mode excitation, which contributes to the significant enhancement of the chirp. Plotted in the figure in blue color are the chirp curves from a "standard" chain with best performance, which was referred to as the "baseline chain". In our research, it was found that sensors with chirp values less than approximately 5 times that of the baseline chain performed well.

\section{b. Sensor calibration}

After confirming that the sensor chain would exhibit satisfactory accuracy and sensitivity, the next step in the procedure was calibrating the chain to a known standard. This process essentially correlates the spectrum at a given temperature with a thermocouple reading. By repeating this process at a number of different points between room temperature and the maximum anticipated operating temperature, it is possible to anticipate the spectrum's behavior through a full range of temperatures. For the field test, where the maximum temperature was expected to be $600^{\circ} \mathrm{C}$, the temperature was stabilized and sampled at around $35^{\circ} \mathrm{C}$ intervals, giving $15-20$ data points.

During calibration, the sensors' optical path differences (OPD) were calculated based on the captured spectrum. For the Fabry-Perot type cavity, OPD of the sensor is twice the product of the effective refractive index of the fiber core mode and the physical length of the cavity, as expressed by

$$
\mathrm{OPD}=2 n_{\mathrm{eff}} L
$$

where $n_{\text {eff }}$ and $L$ represent the fiber cavity index and length, respectively. Both the index and length are dependent on temperature change $(\mathrm{d} T)$

$$
\begin{gathered}
\mathrm{d} L=\alpha L \mathrm{~d} T \\
\mathrm{~d} n_{\text {eff }}=\beta n_{\text {eff }} \mathrm{d} T
\end{gathered}
$$


where $\alpha$ and $\beta$ are the coefficient of thermal expansion (CTE) and thermo-optic coefficient (TOC) of the fiber core material, respectively. Thus, the OPD change is related to temperature change according to

$$
\mathrm{dOPD}=\gamma \mathrm{OPD} \mathrm{d} T
$$

where $\gamma=\alpha+\beta$ is an effective thermal coefficient for OPD change. From Eq. 5.3 we get the important relationship that

$$
d[\ln (\mathrm{OPD})]=\frac{d \mathrm{OPD}}{\mathrm{OPD}}=\gamma d T
$$

with which we concluded that the calibration coefficient for (dOPD/OPD) with respect to $\mathrm{d} T$ is independent on the absolute cavity length, and is purely dependent on the cavity material properties. This leads to an important implication to simplify the calibration process: by calibrating one single sensor to obtain the calibration coefficients for (dOPD/OPD) versus $d T$, such coefficients are applicable for temperature measurement using an IFPI with arbitrary cavity length. Setting $x=\mathrm{dOPD} / \mathrm{OPD}$ and $y=\mathrm{d} T$, the calibration is written as

$$
y=\mathbf{A} \cdot \mathbf{B}^{T}
$$

where $\mathbf{A}=\left[6.3367464 \times 10^{8},-9.3398831 \times 10^{6}, 1.3201338 \times 10^{5}, 1.7157008\right], \mathbf{B}=\left[x^{3}, x^{2}, x, 1\right]$. The universal calibration curve is plotted in Figure 5-8. In obtaining the curve, the value $\mathrm{dOPD} / \mathrm{OPD}_{0}$ was used, where $\mathrm{OPD}_{0}$ is the pre-calculated $\mathrm{OPD}$ at room temperature. With this type of calibration, it is important to accurately determine the value of the vector $\mathbf{A}$. In order to estimate $\mathbf{A}$ with the best quality, two approaches were simultaneously taken. 1) The calibration curves of several best-quality sensors were applied and their average was used to generate the calibration curve and 2) the total phase approach (discussed in Section 3.3.2) was applied for accurate determination of the OPD. 


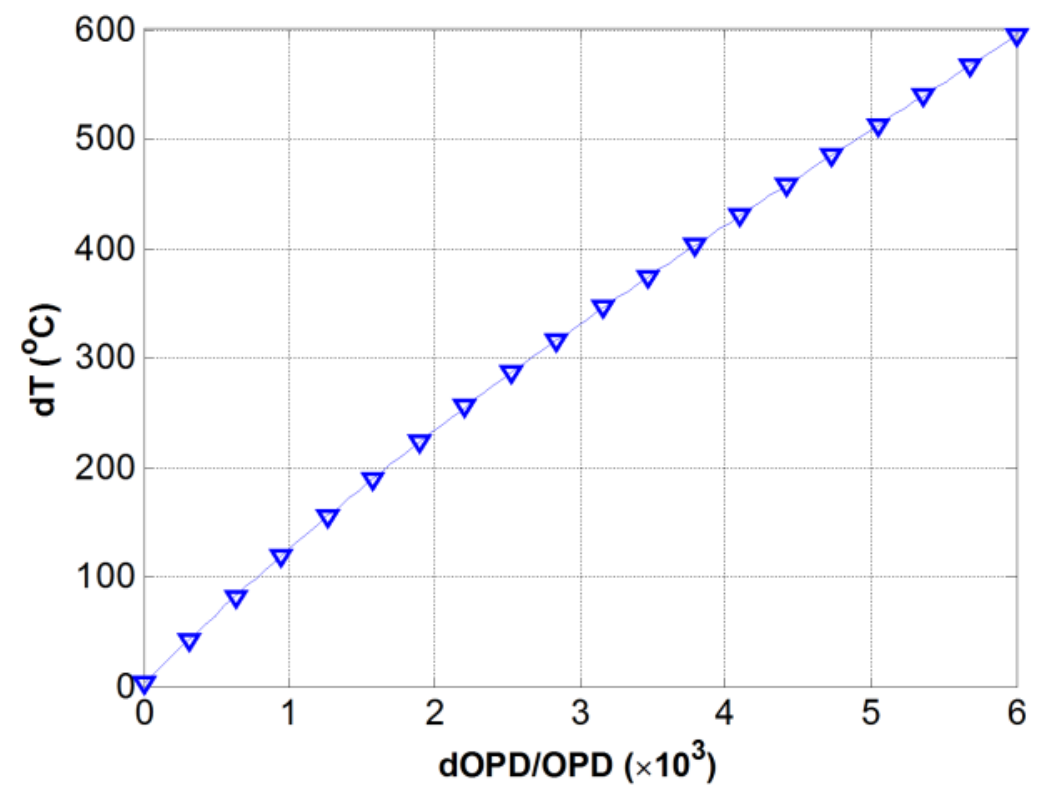

Figure 5-8. The calibration curve (dOPD/OPD) against dT determined with high accuracy for the sensor chain used in the field test.

\subsection{Simulated Environmental Test}

In order to confirm that the proposed design would survive the field-test environment, a chamber, shown in Figure 5-9(a), was fabricated to simulate similar conditions. The coupon with a sensor chain installed sat inside the chamber in a furnace while the temperature was raised to $400^{\circ} \mathrm{C}$. The chamber was then pressurized to $100 \mathrm{psi}$ and the cool air was forced through the holes in the coupon in a manner identical to the combustion chamber located at the NETL. This process is shown in Figure 5-9(b), the dramatic decrease in the measured temperature was due to the injection of the cooling air. With the high speed air flow impinging on the coupon surface, the whole chain was subject to severe vibrations. Through the test, we confirmed the following: 1) the strong vibration of the sensor would not affect the signal quality and 2) the air flow would not induce potential breakage at 100psi. Subsequent tests were run at temperatures up to $650^{\circ} \mathrm{C}$, at which point the aluminum chamber melted. However, it was confirmed that the sensor chains were robust enough, and capable of withstanding high temperatures while still producing accurate results. 

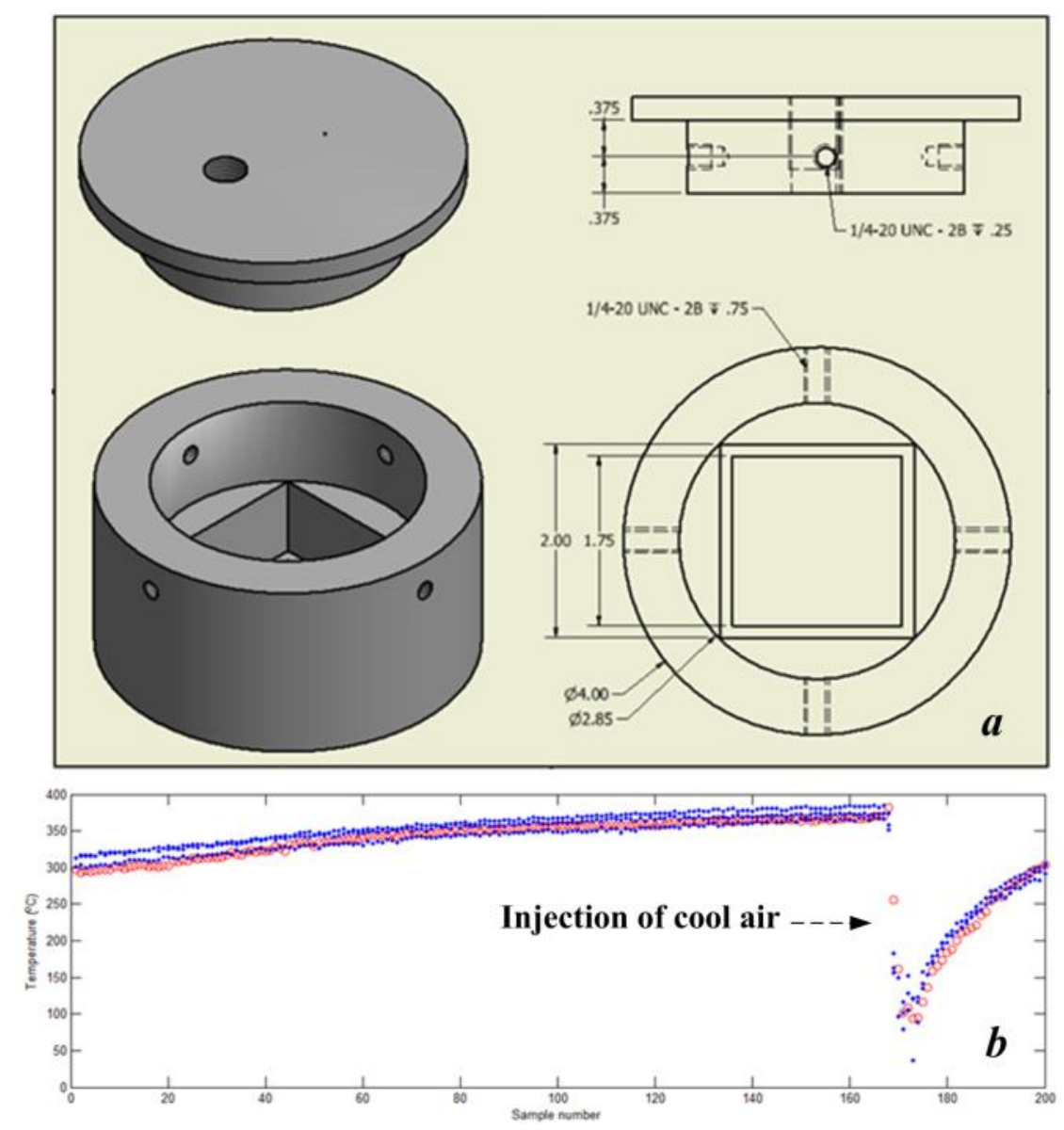

Figure 5-9. (a) Mechanical drawing of the testing environmental chamber design. (b) the measured temperature using an installed sensor chain during the testing process.

\subsection{Sensor installation}

a. Design of the assembly

The assembly required to attach the fiber feedthrough to the existing system was constructed according to dimensioned drawings provided by the NETL. Figure 5-10 shows the final assembly. The pressure-rated fiber feedthrough can be clearly seen on the right-hand-side of the assembly. The tubing provides working space for fiber splices. Due to concerns about high-velocity air and its impact on the survivability of the unprotected fiber inside the 1" tubing in the center, a perforated plug was installed into the left-most side of the tubing. The fiber will be fed through the center hole in the plug, and the relatively tight tolerances should serve to minimize fiber 
vibration. The holes drilled around the circumference of the plug will eliminate a pressure difference across the plug, which eliminates the danger associated with using an unrated and untested pressure boundary.

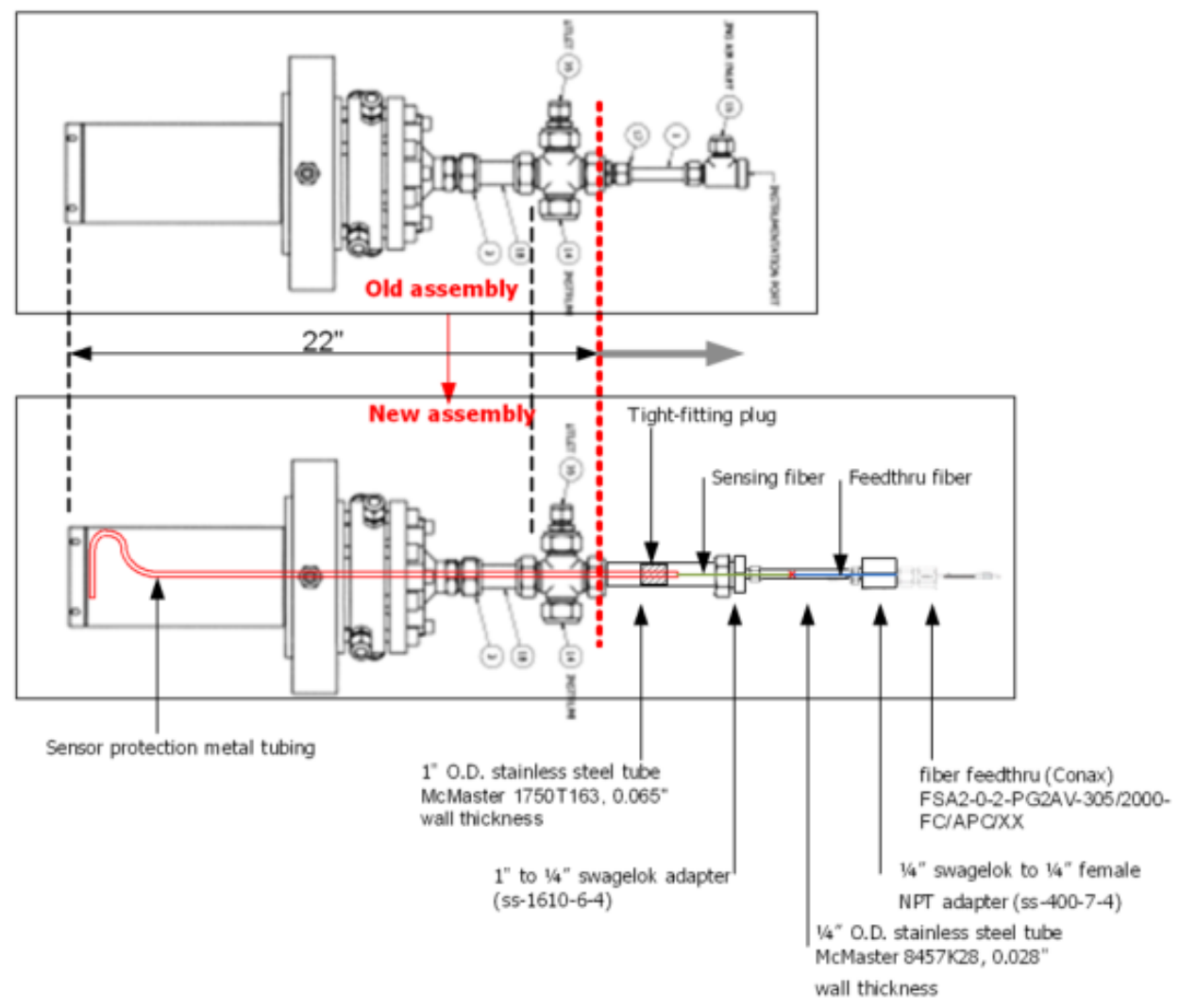

(a)

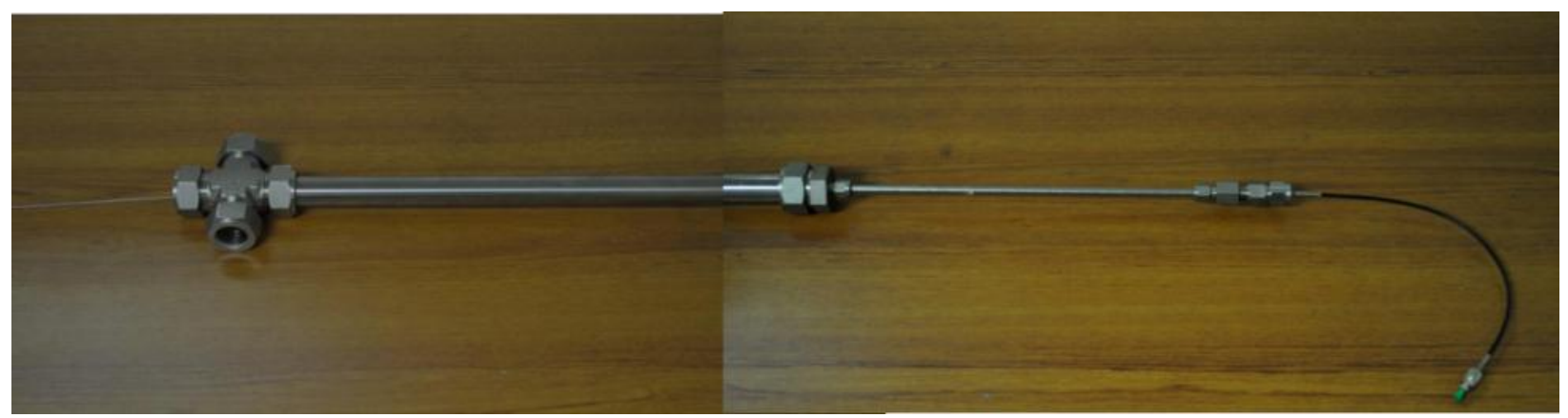

(b)

Figure 5-10. (a) Design of the optical fiber feedthrough assembly. (b) Feedthrough assembled in the lab. 
The metal plug was machined in our mechanical room, with the O.D. slightly larger than the I.D. of the 1" O.D. tubing. A central bore of the size slightly larger than the O.D. of the protection metal tubing was drilled for fiber stabilization. To install the plug into the outer tube, the tube was heated prior to insertion of the plug, to thermally expand its dimension. After heating, the plug can be inserted into the outer tube, and fixed very firmly when the tube cooled down. Fiber fusion splicing will be performed between the fiber intruding from the 1" tube and the fiber connected to the feedthough (both fibers are with redundant lengths). They will then be cut into desired lengths and spliced. The extra fiber will be curled inside the 1 " tube, and the tube will be finally fastened with the tube adapter. Figure 5-11(a) illustrates the design for the plug, and its relative position with the fiber and outer tubing. Figure 5-11(b) is a photograph showing the machined plug installed inside the 1 " tube.

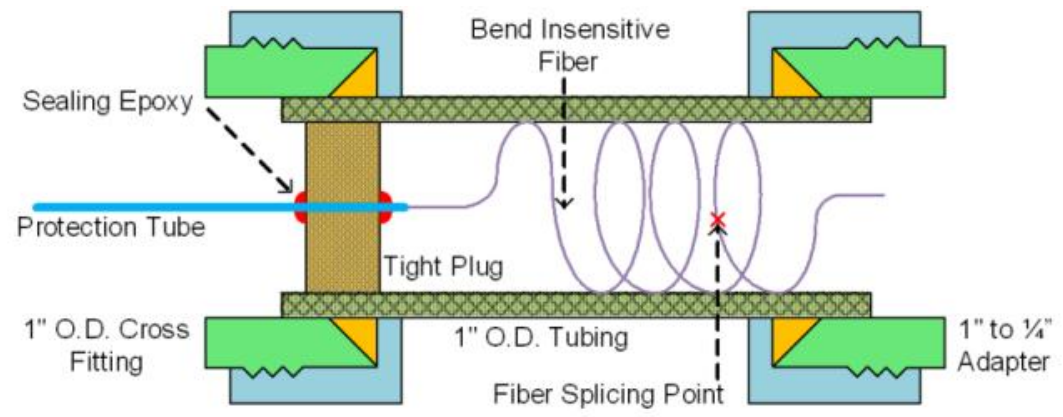

(a)

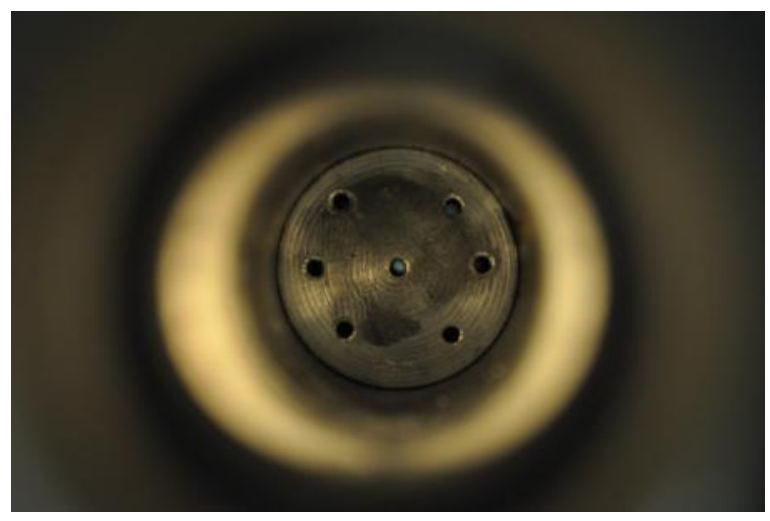

(b)

Figure 5-11. (a) Design of the 1" O.D. tube with the fitting plug. The plug provides stabilization of the metal tubing, and the bend-insensitive fiber will be curled inside the tubing. (b) Cross-sectional view showing the machined plug. The central bore is for the fiber to go through, while the surrounding holes provide air-path to maintain balanced pressure on both sides of the plug. 
b. Design of installation procedure

Installing the entire setup onto the existing system will be according to the following procedure:

1. Insert fiber and sensor chain into a section of stainless steel microtubing for protection

2. Route the fiber and protective tube through the fiber hold downs

3. Install the coupon and fiber sensors into the the coupon holder

4. Run the optical fiber and protective tube up through the existing chambers, through the antivibration plug, and then through the new tubing assembly until the end of the fiber passes completely through the .25 " tubing which interfaces with the (disconnected) commercial fiber feedthrough

5. The stainless steel protective tubing will end $\sim 3 \mathrm{~cm}$ to the right of the anti-vibration plug

6. Splice the end of the fiber with the fiber coming out of the commercial fiber feedthrough

7. Carefully attach the fiber feedthrough to the .25 " tubing while coiling the excess fiber into the 1 " tubing.

8. Tighten all fittings and test for leaks.

c. Field installation

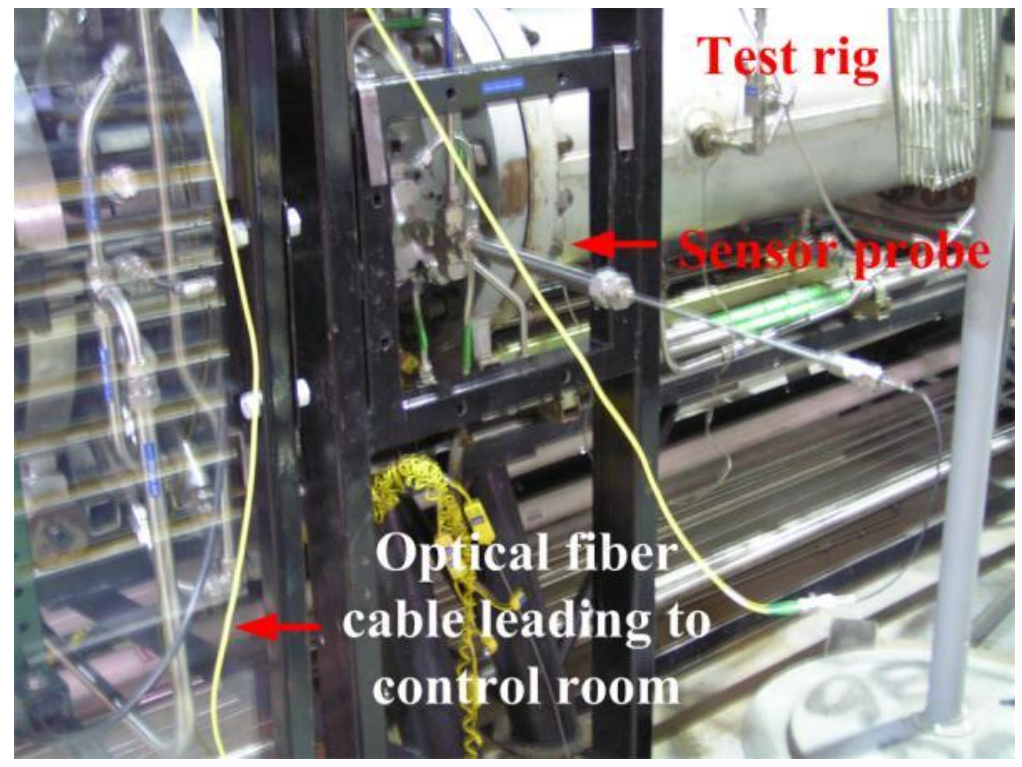

Figure 5-12. Photo of the installed fiber optic probe (photo taken during the field test). 
Upon arrival at the NETL on Nov. $15^{\text {th }}, 2011$, a sensor chain was installed in the system. This chain was routed in configuration 2, i.e. multiple loops routed through the hold-down tubes. Unfortunately, upon installation this chain broke, and replaced with the final sensor chain. This chain was routed in configuration 3, i.e. free-form routing. The following morning this sensor chain was subjected to a battery of tests and harsh operating conditions, including temperatures up to $700^{\circ} \mathrm{C}$. The installed assembly is shown in Figure 5-12.

\subsection{Testing results}

The field test started at 9:30 a.m., November $16^{\text {th }}, 2011$. The test lasted for 5.5 hours, during which the maximum coupon temperature reached beyond $700^{\circ} \mathrm{C}$. The sensor signal was remotely interrogated with a 10-meter SMF28 (Corning Inc.) optical fiber cable, which was connected to the Component Testing System (Model Si-720, Micron Optics, Inc.), we used toggle rate of $5 \mathrm{~Hz}$ (rather than $0.5 \mathrm{~Hz}$ ) to minimize the scanning-rate induced additional phase term in the spectrograph. The spectral interrogation unit interfaced with a laptop (Lenovo, Edge 14) through a General Purpose Interface Bus (GPIB) cable. Data acquisition and processing were performed with a Matlab based platform. Signal demodulation was performed in real-time using the direct OPD estimation algorithm introduced in Section 3.3.2 (b), Eq. 3.48. The universal calibration coefficient in Eq. 5.5 was used to calculate the temperature. During the field test, the cooling air flow rate and the gas pressure in the rig were adjusted during different periods, giving rise to controlled temperature change on the coupon surface (See Table 5.4 ).

Table 5.4. Parameters affecting the coupon temperature during the field test.

\begin{tabular}{c|c|c|c}
\hline \hline Parameter & Minimum & Maximum & Relationship with temperature \\
\hline $\begin{array}{c}\text { Rig pressure } \\
\text { (psig) }\end{array}$ & 1.54 & 30.48 & Temperature increases with rig pressure \\
\hline $\begin{array}{c}\text { Cooling air flow rate } \\
(\mathrm{scfh})\end{array}$ & 48.85 & 400 & $\begin{array}{c}\text { Temperature decreases with cooling air } \\
\text { flow rate }\end{array}$ \\
\hline $\begin{array}{c}\text { Total air flow rate } \\
(\mathrm{x} \mathrm{104} \mathrm{scfh})\end{array}$ & 0.2982 & 3.3449 & $\begin{array}{c}\text { Temperature increases with total air } \\
\text { flow rate }\end{array}$ \\
\hline
\end{tabular}


The real-time monitored temperatures measured by the sensors were co-plotted in Figure 5-13. The larger noise depicted in the figure is due to the poor resolution associated with the direct OPD estimation algorithm. However, the measured raw temperature delivered some important information: 1) the temperature changes measured showed excellent agreement with the inner rig temperature measured with thermocouple. 2) the sensors demonstrated significant temperature gradient across the coupon. The sensor at the middle far from the air holes (black) showed the highest temperature, during the entire test. With cooling air, the sensor in the middle closest to the air holes (pink) had the lowest temperature when cooling air was turned on, where as the temperature measured by the sensors at two sides (blue and red) were the lowest when cooling air was turned off. The maximum temperature gradient was almost $100^{\circ} \mathrm{C}$ across the length from the middle to the edge of the coupon. This trend was in agreement with expectation and with the temperature map recorded with a pyrometer located at the back side of the coupon (inner temperature). The raw data was post-processed with the total phase demodulation algorithm (see Section 3.3.2 b, Eq. 3.50). Post-processing corrected several phase jumps associated with this more accurate demodulation algorithm. As depicted in Figure 5-14, the data calculated using the total phase algorithm demonstrated significant noise reduction, which better exposed the details of the temperature history. In the same figure, the measured values of the parameters listed in Table 5.4 were co-plotted with the same time scale to better expose their correlations.

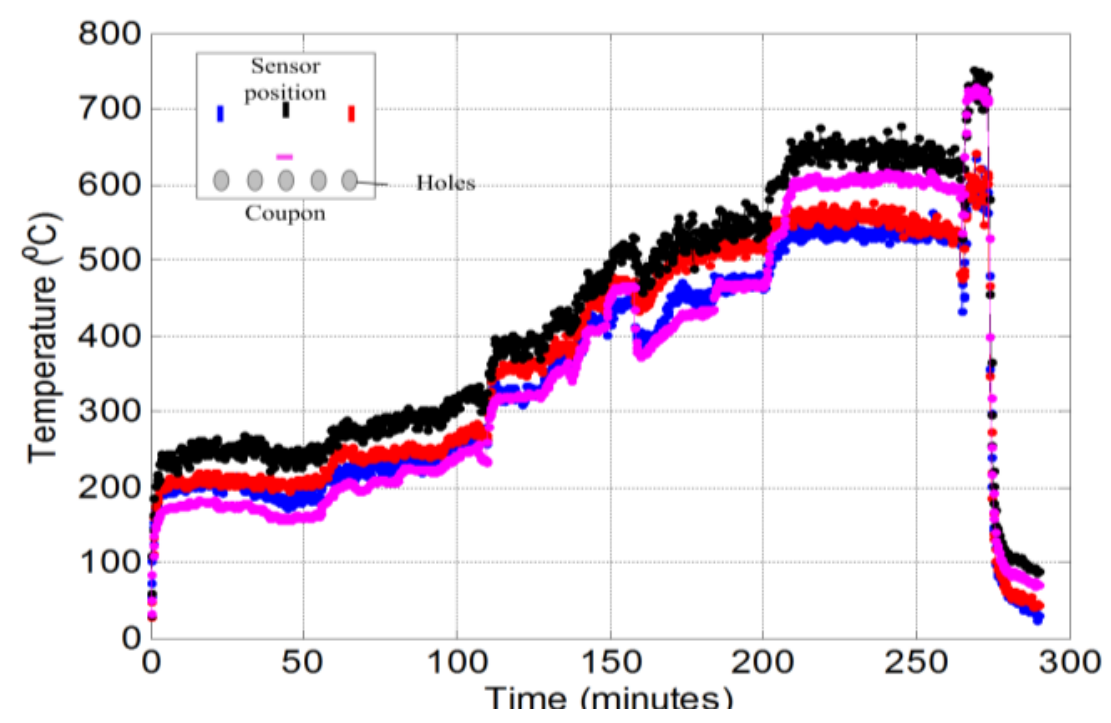

Figure 5-13. Measured temperature (real time) with direct OPD demodulation algorithm. 

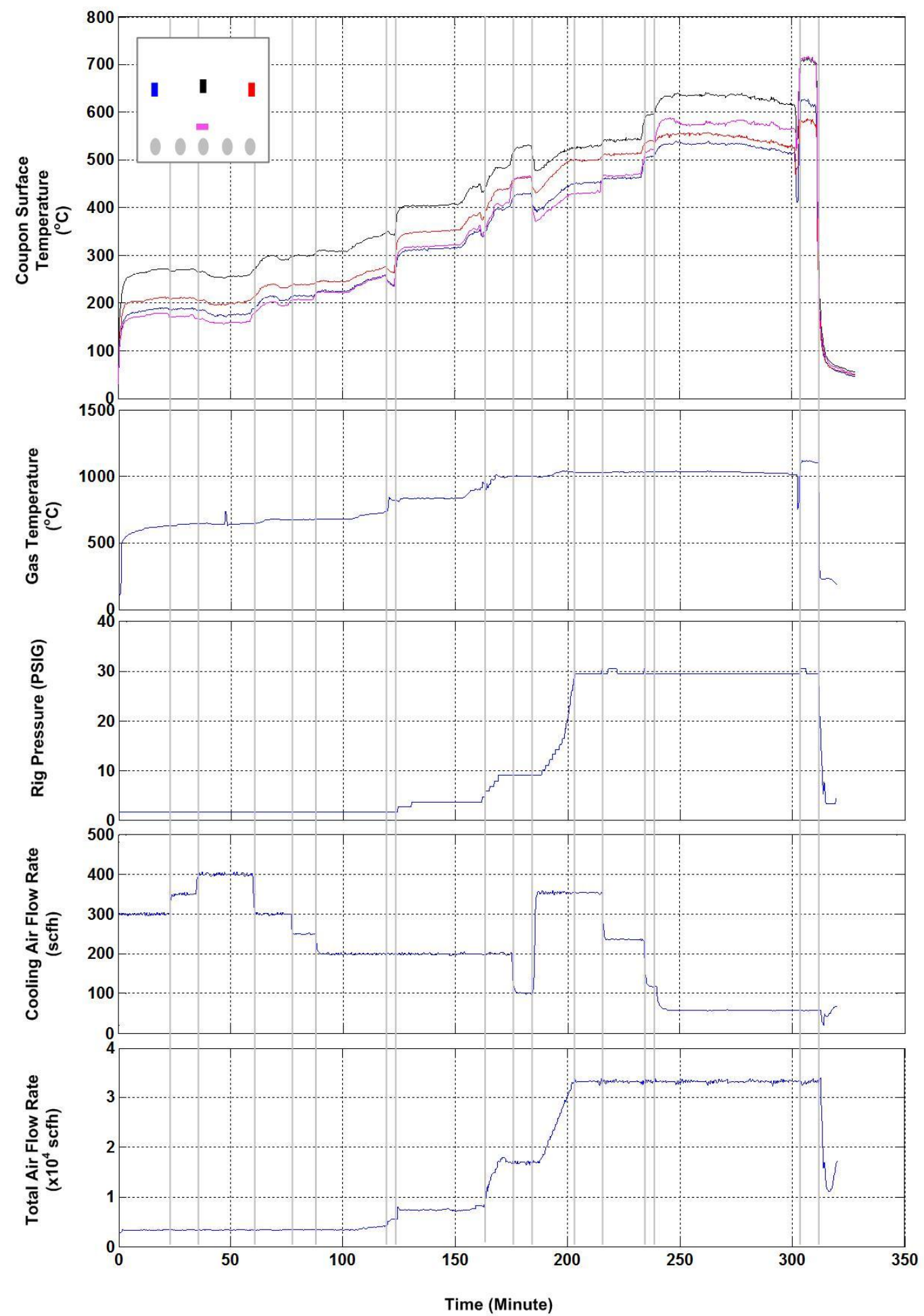

Figure 5-14. Post-processed data with the total phase algorithm. The control parameters are plotted with the same time scale for better comparison. 


\section{CONCLUSION}

The three-year program aims at developing a multiplexed fiber optic IFPI sensing system for health monitoring for ultra-supercritical boilers. The main investigations are focused on sensor design, fabrication, attachment techniques and novel materials for high temperature and strain measurements.

The sensor design involves splicing condition optimization, transmission loss reduction, sensor signal demodulation and sensor system design. Through our analysis and experiments, we achieved balanced performance in reflectivity and mechanical strength by optimizing the splicing condition; the transmission loss was reduced from $3 \mathrm{~dB}$ on average to less than $0.5 \mathrm{~dB}$ by a novel cavity refocusing approach; a new total phase method was proposed to address signal demodulation issues with increased measurement accuracy; a system with significantly improved multiplexibility and reliability was resulted from the analysis and optimizations.

The sensor has been attached to a secondary material before attaching to the waterwall tube therefore, thermal strain from the waterwall tube has been largely reduced and the sensor was able to measure the strain successfully in the proposed temperature range. Strain compensation technique was also tested in order to design sensors to measure even larger strain. Gold-coated fibers were used as the sensor material in order to enhance the fiber/sensor mechanical strength. A simulated testing environment was designed and developed in order to calibrate and test the sensors, in which the strain can be applied to the sensor with calculated values. In the meantime, the system temperatures can also be changed according to the project requirements.

A sensor link with five temperature sensors and a single strain sensor was tested in the simulated environment in the laboratory. The temperature sensors demonstrated measurement range from room temperature to $800^{\circ} \mathrm{C}$ at an accuracy of $2.0{ }^{\circ} \mathrm{C}$ and a resolution of $0.05^{\circ} \mathrm{C}$. The strain sensor showed a strain accuracy of $3.8 \mu \varepsilon$ and a resolution of $3.0 \mu \varepsilon$, from room temperature to $600^{\circ} \mathrm{C}$. The testing results met the technical requirements we determined with Alstom Power at the start of the project. A field test was successfully conducted at NETL in which four IFPI sensors yielded distributed temperature mapping of a testing coupon installed in the turbine test rig, the test lasted for five hours and the highest temperature exceeded $700^{\circ} \mathrm{C}$. The measurement results agreed well with prior results generated with thermocouples. 


\section{REFERENCE}

1. Choi, H.S.; Taylor, H.F.; Lee, C.E. High-performance fiber-optic temperature sensor using low-coherence interferometry. Opt Lett 1997, 22, 1814-1816.

2. Lee, C.E.; Taylor, H.F. Interferometric Optical Fiber Sensors Using Internal Mirrors. Electron Lett 1988, 24, 193-194.

3. Tseng, S.M.; Chen, C.L. Optical Fiber Fabry-Perot Sensors. Appl Optics 1988, 27, 547551.

4. Huang, Z.Y.; Zhu, Y.Z.; Chen, X.P.; Wang, A.B. Intrinsic Fabry-Perot fiber sensor for temperature and strain measurements. Ieee Photonic Tech L 2005, 17, 2403-2405.

5. Kersey, A.D.; Dandridge, A.; Tveten, A.B. Time-Division Multiplexing of Interferometric Fiber Sensors Using Passive Phase-Generated Carrier Interrogation. Optics Letters 1987, 12, 775-777.

6. Kersey, A.D. Demonstration of a Hybrid Time/Wavelength Division Multiplexed Interferometric Fiber Sensor Array. Electronics Letters 1991, 27, 554-555.

7. Kullander, F. Code division multiplexing in interferometric optical fiber sensor networks. Optical Fiber Sensors Conference Technical Digest 2002, 1, 523.

8. Hu, Y.Q.; Chen, S.P. Two-dimensional encoding scheme for digital spatial domain multiplexed large-scale optical fiber sensor arrays. Ieee Transactions on Instrumentation and Measurement 1997, 46, 1160-1164.

9. Brooks, J.L.; Wentworth, R.H.; Youngquist, R.C.; Tur, M.; Kim, B.Y.; Shaw, H.J. Coherence Multiplexing of Fiber-Optic Interferometric Sensors. Journal of Lightwave Technology 1985, 3, 1062-1072.

10. Yablon, A.D. Optical fiber fusion splicing; Springer: Berlin; New York, 2005; p.^pp.

11. Froggatt, M.; Moore, J. High-Spatial-Resolution Distributed Strain Measurement in Optical Fiber with Rayleigh Scatter. Appl. Opt. 1998, 37, 1735-1740.

12. Emkey, W.; Jack, C. Analysis and evaluation of graded-index fiber lenses. Lightwave Technology, Journal of 1987, 5, 1156-1164.

13. Ghatak, A.K.; Thyagarajan, K. An introduction to fiber optics; Cambridge University Press: Cambridge; New York, 1998; p.^pp.

14. Wang, Q.; Farrell, G.; Yan, W. Investigation on Single-Mode-Multimode-Single-Mode Fiber Structure. J. Lightwave Technol. 2008, 26, 512-519.

15. Feit, M.D.; Fleck, J.J.A. Light propagation in graded-index optical fibers. Appl. Opt. 1978, 17, 3990-3998.

16. Zhang, Y.; Li, Y.; Wei, T.; Lan, X.; Huang, Y.; Chen, G.; Xiao, H. Fringe Visibility Enhanced Extrinsic Fabry-Perot Interferometer Using a Graded Index Fiber Collimator. Photonics Journal, IEEE 2010, 2, 469-481.

17. Ma, C.; Lally, E.M.; Wang, A. Toward Eliminating Signal Demodulation Jumps in Optical Fiber Intrinsic Fabry-Perot Interferometric Sensors. J. Lightwave Technol. 2011, 29, 1913-1919.

18. Zhu, Y.; Huang, Z.; Shen, F.; Wang, A. Sapphire-fiber-based white-light interferometric sensor for high-temperature measurements. Opt. Lett. 2005, 30, 711-713.

19. Ma, C.; Wang, A. Multimode excitation-induced phase shifts in intrinsic Fabry?Perot interferometric fiber sensor spectra. Appl. Opt. 2010, 49, 4836-4845. 
20. Chen, S.; Palmer, A.W.; Grattan, K.T.V.; Meggitt, B.T. Digital signal-processing techniques for electronically scanned optical-fiber white-light interferometry. Appl. Opt. 1992, 31, 6003-6010.

21. Qi, B.; Pickrell, G.R.; Xu, J.; Zhang, P.; Duan, Y.; Peng, W.; Huang, Z.; Huo, W.; Xiao, H.; May, R.G.; Wang, A. Novel data processing techniques for dispersive white light interferometer. Optical Engineering 2003, 42, 3165-3171.

22. Shen, F.; Wang, A. Frequency-estimation-based signal-processing algorithm for whitelight optical fiber Fabry-Perot interferometers. Appl. Opt. 2005, 44, 5206-5214.

23. Jiang, Y. High-resolution interrogation technique for fiber optic extrinsic Fabry-Perot interferometric sensors by the peak-to-peak method. Appl. Opt. 2008, 47, 925-932.

24. Han, M.; Zhang, Y.; Shen, F.; Pickrell, G.R.; Wang, A. Signal-processing algorithm for white-light optical fiber extrinsicFabry-Perot interferometric sensors. Opt. Lett. 2004, 29, 1736-1738.

25. Tretter, S. Estimating the frequency of a noisy sinusoid by linear regression Information Theory, IEEE Transactions on 1985, 31, 832-835. 


\title{
APPENDIX I. MATLAB CODE FOR OPD DEMODULATION
}

\author{
Matlab code for OPD demodulation using peak-tracking method.
}

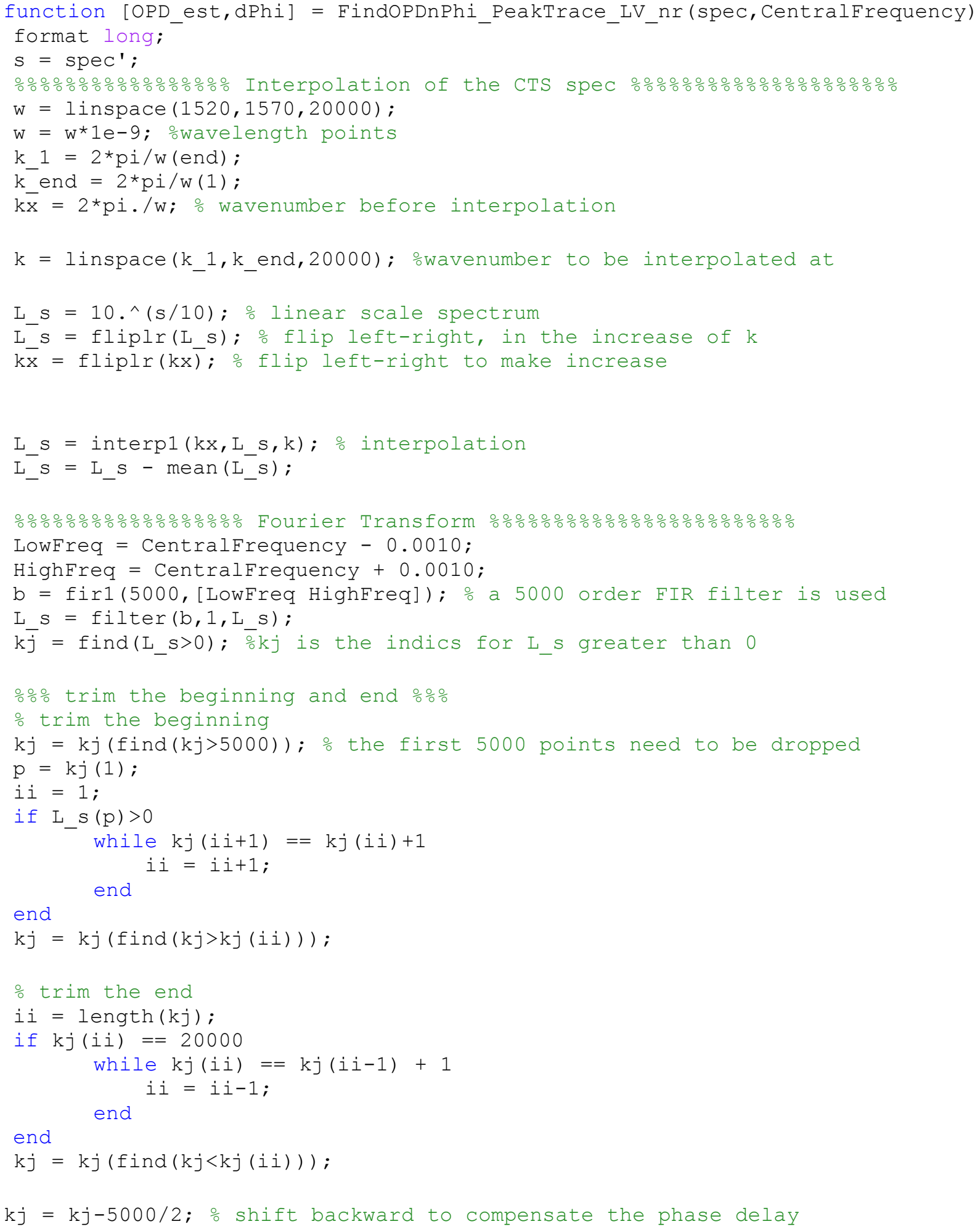




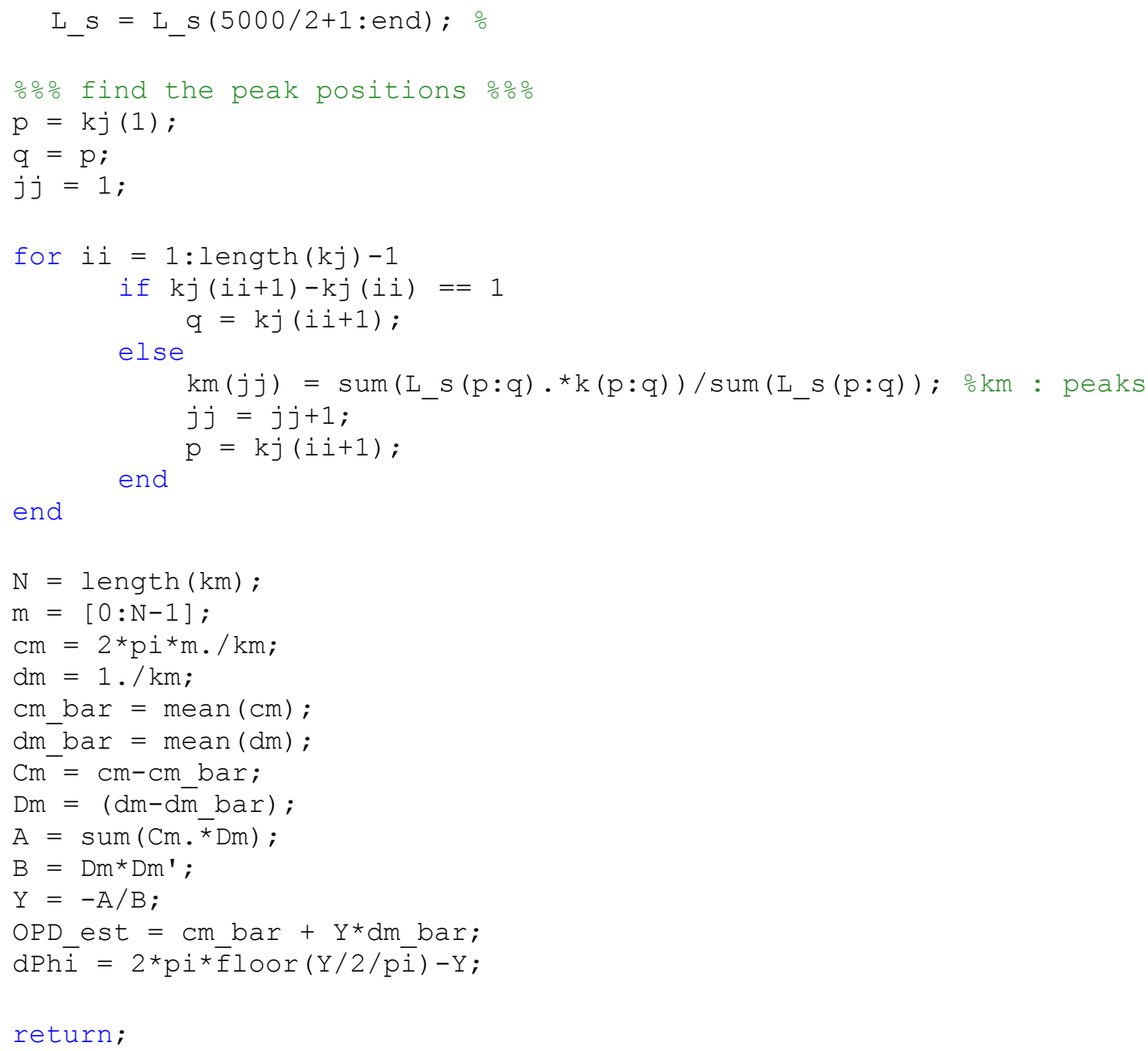


APPENDIX II. ALSTOM POWER TECHNICAL REPORT 


\title{
ALSTOM
}

Technical Report

Document Title:

\section{Examination of Various Strain Gauge Attachments after Corrosion Testing}

Document Ref. No.:

$11 \mathrm{E} 216$

Date of Issue:

December 5, 2011

No. of pages: 25

Client:

Author(s):

\author{
Leo Antony \\ TTTM, Materials and Materials Processing \\ 1201 Riverfront Parkway \\ Chattanooga, TN 37402 \\ Tel.: (423) 752-2845 \\ Fax: (423) 752-2825 \\ leo.antony@power.alstom.com
}

Distribution: Joseph Quinn

Keywords:

Strain Gauge Attachments, Corrosion, Oxidation and Sulfidation, Inconel Strips, Ceramic Adhesives, Capacitive Discharge Welds

Reviewed by:

Joseph Quinn

Summary:

Metallurgical examination of the test specimen after corrosion testing at $600^{\circ} \mathrm{C}$ and $650^{\circ} \mathrm{C}$ revealed that the Inconel strips attached onto the substrate by capacitive weld technique did not show any detachment; however, there is a concern in terms of long term serviceability because of the corrosion product formation underneath the Inconel strips/between the weld locations onto the substrate. The Inconel strips that were attached onto the substrate using Ceramabond 671-1112 and Resbond 989FS adhesives did show detachment from the substrate for both the test conditions and thus the performance was judged to be unsatisfactory.

The maximum corrosion product thickness observed on the test specimen/T22 material after $650^{\circ} \mathrm{C}$ test conditions was approximately 29 mils, which is approximately three times more than the $600^{\circ} \mathrm{C}$ test conditions.

A more corrosion resistant Inconel-690 material can be substituted for Inconel-600, which was used in this design, to combat the Inconel strip corrosion issues.

Information disclosed herein is furnished to the recipient solely for the use thereof as has been agreed upon with Alstom and all rights to such information are reserved by Alstom.

The recipient of the information disclosed herein agrees, as a condition of its receipt of such information, that the Power Plant Laboratories of Alstom Power Inc. shall have no liability for any direct or indirect damages including special, punitive, incidental, or consequential damages caused by, or arising from, the recipient's use or non-use of the information. 


\section{INTRODUCTION}

As a part of Ultra Super Critical (USC) Boiler Advanced Instrumentation Project, four test specimens were sent to the TTTM lab for boiler fireside corrosion testing and subsequent metallographic evaluation to assess the long-term survivability of the strain gauge attachments that were included with the specimens. Each test specimen contained four Inconel strips that were attached by different techniques onto the T22 test specimen, as described in Figure 3.

The purpose of the analysis was to document and evaluate the condition of the attachments and specimens after corrosion testing in a simulated furnace environment.

\section{CORROSION TESTING}

The test specimens were coated with the ash deposit, placed in ceramic boats (see Figure 2), and then inserted into horizontal tube furnaces (see Figure 1) that were operated at $600^{\circ} \mathrm{C}$ (2 specimens) and $650^{\circ} \mathrm{C}$ (2 specimens) for 30 days. The atmosphere consisted of a simulated eastern US coal combustion flue gas. The chemical composition of the simulated flue gas was as shown below:

$\mathrm{N}_{2}$ (balance), $15 \% \mathrm{CO}_{2}, 10 \% \mathrm{H}_{2} \mathrm{O}, 3.6 \% \mathrm{O}_{2}, 0.2 \% \mathrm{SO}_{2}, 0.01 \% \mathrm{Cl}$

The composition of the simulated ash deposit was as shown in Table 1 below:

Table 1. Ash Composition

\begin{tabular}{|c|c|}
\hline Composition & Composition, $\%$ \\
\hline $\mathrm{SiO}_{2}$ & 41.6 \\
\hline $\mathrm{Al}_{2} \mathrm{O}_{3}$ & 20.0 \\
\hline $\mathrm{Fe}_{3} \mathrm{O}_{4}$ & 30.8 \\
\hline $\mathrm{Na}_{2} \mathrm{SO}_{4}$ & 2.5 \\
\hline $\mathrm{K}_{2} \mathrm{SO}_{4}$ & 2.5 \\
\hline Carbon & 2.5 \\
\hline Methyl & +1.66 grams $/ 100$ \\
Cellulose & grams of ash \\
\hline
\end{tabular}

The schematic of the experimental set-up is shown in Figure 2.

At the completion of the test, the furnaces were cooled to room temperature and the test specimens removed. These specimens were then evaluated metallographically in terms of the corrosion product thickness and also the condition of the attachment. 


\section{SUPPORTING DOCUMENTATION}

\subsection{Visual Examination}

The test specimens were visually inspected after the corrosion testing to see if there were any signs of corrosion damage and/or attachment failures. In order to observe the condition of the test specimens more carefully, the ash deposits were removed from the surfaces. Upon inspection (as shown in Figures 6 \& 7), the Inconel strips attached by the ceramic adhesive methods appeared to be detached from the specimens for both test temperatures; 600 and $650{ }^{\circ} \mathrm{C}$.

\subsection{Metallography}

Metallographic specimens were removed from selected locations on the specimens so that their macrostructural and microstructural features could be evaluated in detail using light microscopy. The approximate locations from where metallographic sections were taken are indicated in Figures 6 \& 7.

The condition of the ID and OD surfaces of the test specimen, after corrosion testing at $600^{\circ} \mathrm{C}$, are shown in Figures 8 through 13. It was evident that the Inconel strips were intact for when the capacitive weld was used; however, there was corrosion product ( 2 mils) seen beneath the Inconel strip in between the weld spots. This corrosion could pose an issue regarding long-term serviceability. The Inconel strip was corroded where Resbond 989FS was used (as shown in Figure 10) and that an SEM-EDS analysis was performed on the corrosion product that was observed along the edges of the Inconel strip where Resbond989FS had been used (see Figure 19). The finding suggests that corrosive species of oxygen and sulfur were present, indicative of oxidation-sulfidation mechanism. The Inconel strip was consistent with the composition of Inconel-600 and the specimen was consistent with T22 material.

The condition of the ID and the OD surfaces of the test specimens after corrosion testing at $650^{\circ} \mathrm{C}$ are shown in Figures 14 through 17 . The condition of the attachments at this temperature was similar to those tested at $600^{\circ} \mathrm{C}$.

The maximum corrosion product thickness observed on the specimens after corrosion testing at $600^{\circ} \mathrm{C}$ and $650^{\circ} \mathrm{C}$ are shown in Figures 13 and 18 . The maximum corrosion product thickness observed after testing at $650^{\circ} \mathrm{C}$ was approximately 29 mils, which is approximately three times more than that which was observed at the $600^{\circ} \mathrm{C}$ test conditions. 


\section{CONCLUSIONS}

Metallurgical examination of the test specimens after corrosion testing at $600^{\circ} \mathrm{C}$ and $650^{\circ} \mathrm{C}$ revealed that the capacitive discharge weld attachments did not separate from the specimens. It should be noted, however, that corrosion products were seen underneath the Inconel strip, a finding which may pose an issue for long-term serviceability.

The Inconel strips that were attached onto the specimens using Ceramabond 671-1112 and Resbond 989FS adhesives were detached.

SEM-EDS analysis of the corrosion product on the Inconel strips confirmed that the corrosion mechanism was oxidation-sulfidation. The Inconel strip material was identified as Inconel-600, while the specimens were T22 material.

The Inconel-600 strips showed significant corrosion damage at both test temperatures. As such, it is recommended to use a more corrosion resistant material (with a chromium content $>25 \%$ ) such as Inconel 690 .

The maximum corrosion product thickness observed on the test specimen/T22 material after $650^{\circ} \mathrm{C}$ test conditions was approximately 29 mils, which is approximately three times more than the $600^{\circ} \mathrm{C}$ test conditions. 

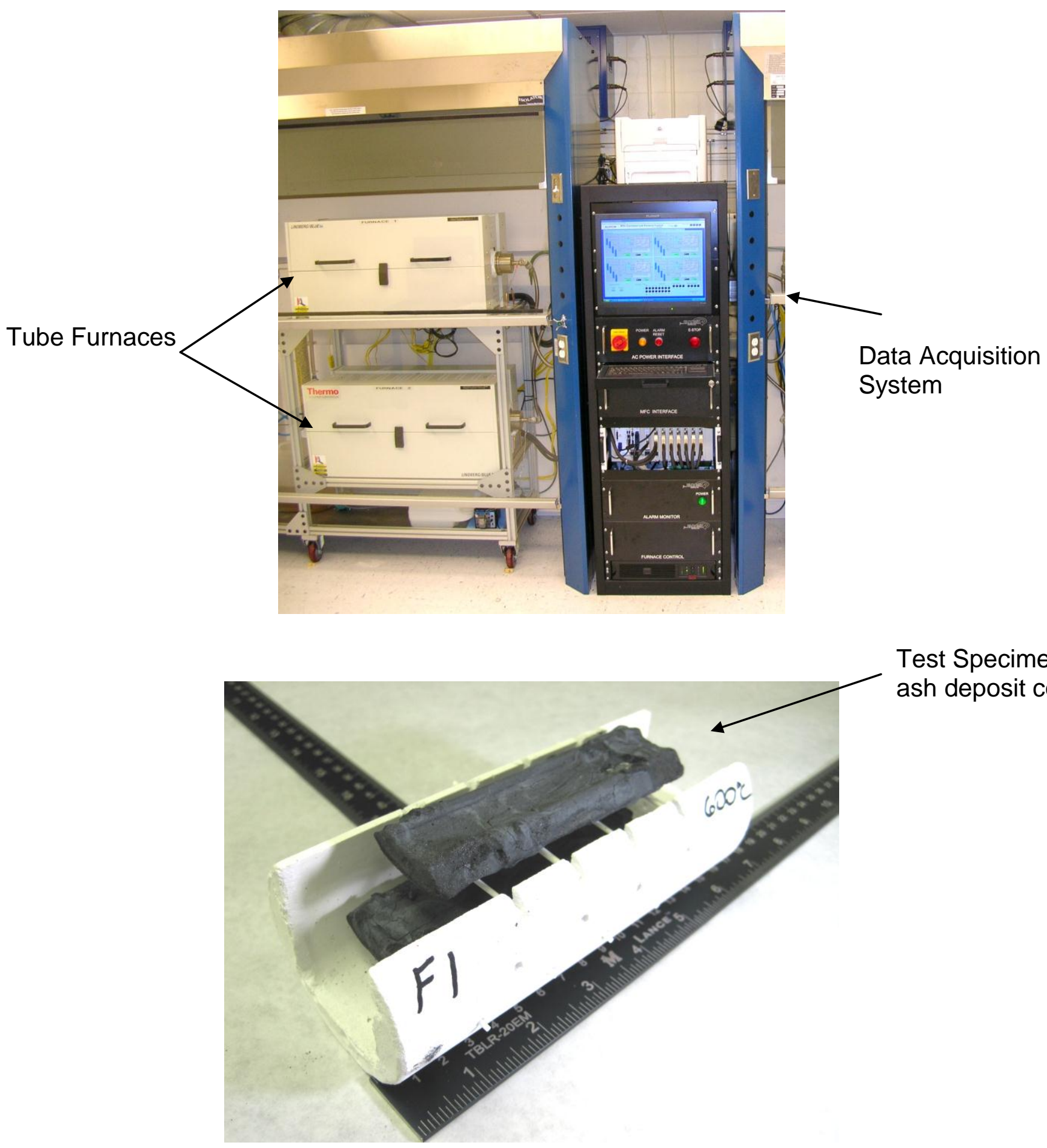

Test Specimen (after ash deposit coat)

Figure 1. The top photo shows the tube furnaces that were used for the testing, while the bottom photo shows one of the ceramic boats with the test specimen (after ash deposit coat). 


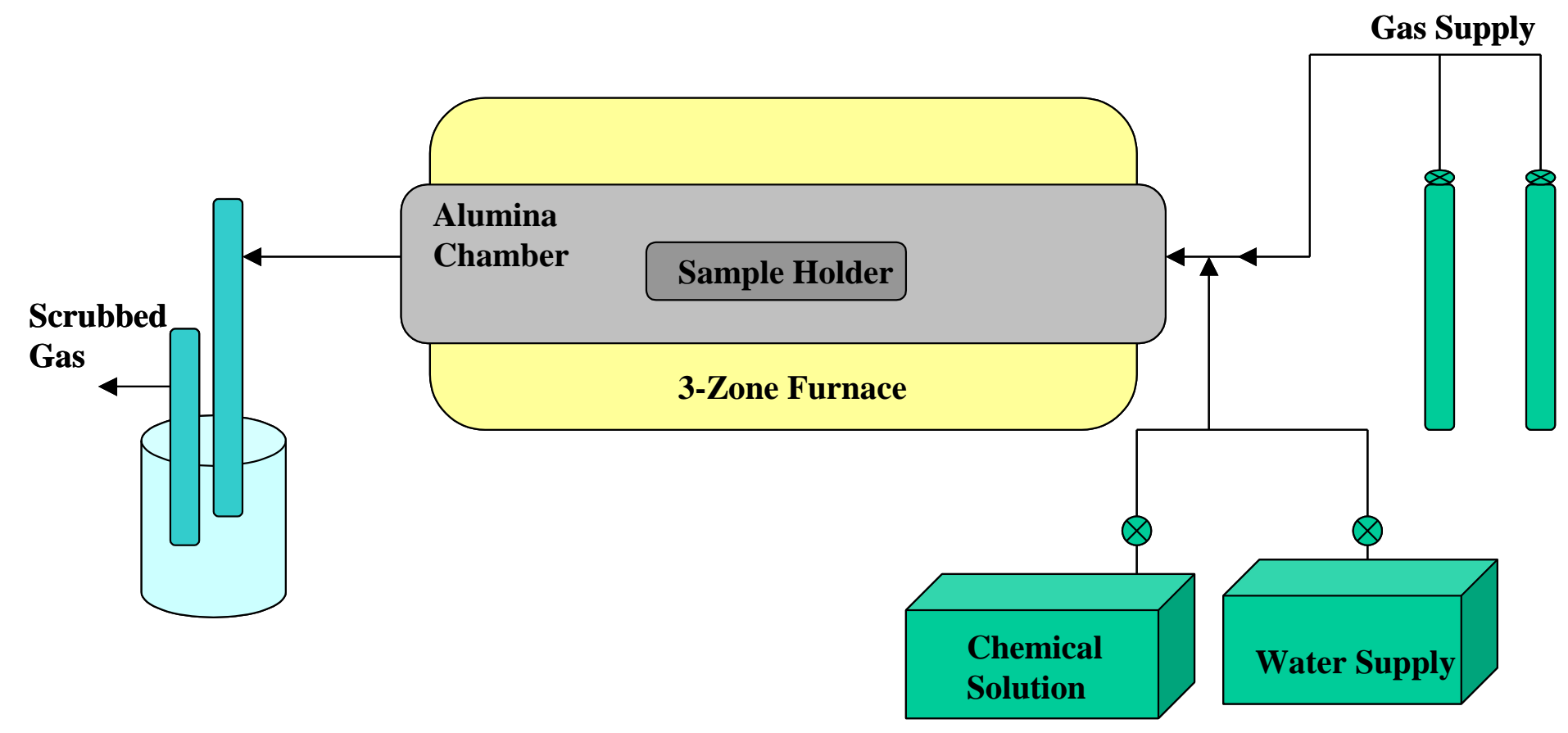

Figure 2. Shown is the schematic of the experimental set-up. 

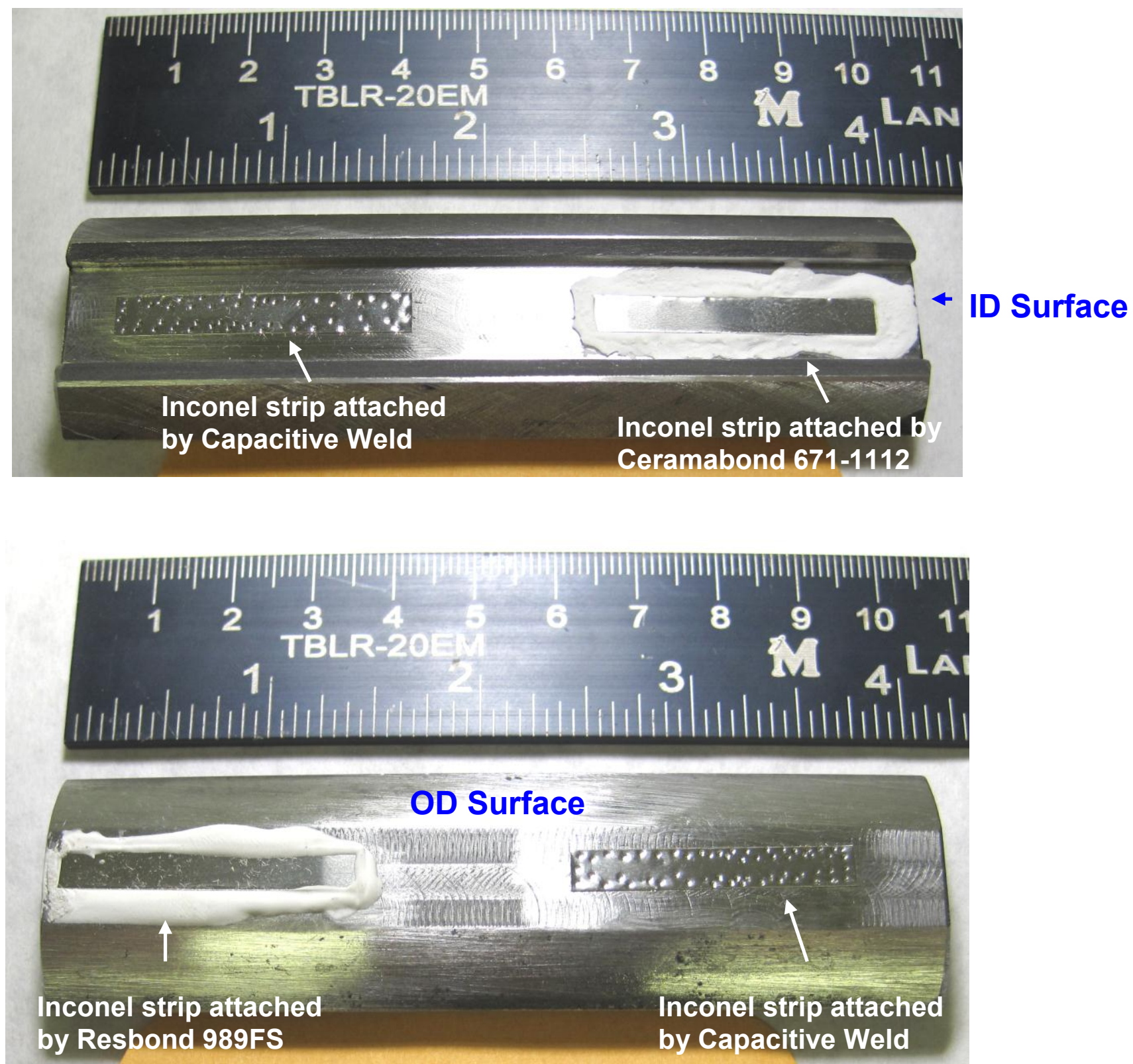

Figure 3. The typical views of the test specimen on the ID and OD surfaces are shown, revealing four Inconel strips that were attached onto the substrate by different attachment methods. 

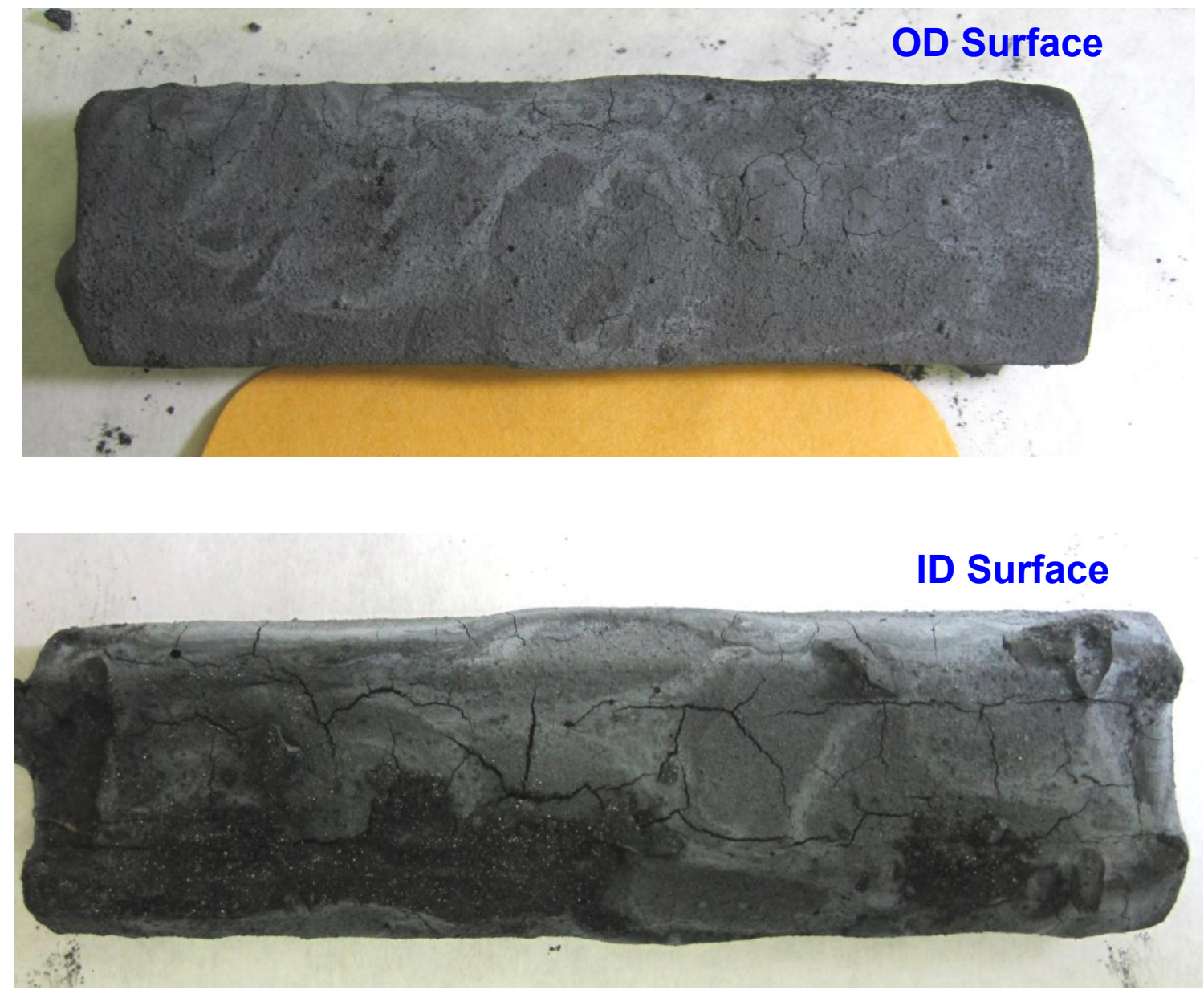

Figure 4. The typical views of the test specimen (ID and OD surfaces) after the ash deposit coating (prior to the corrosion testing) are shown. 


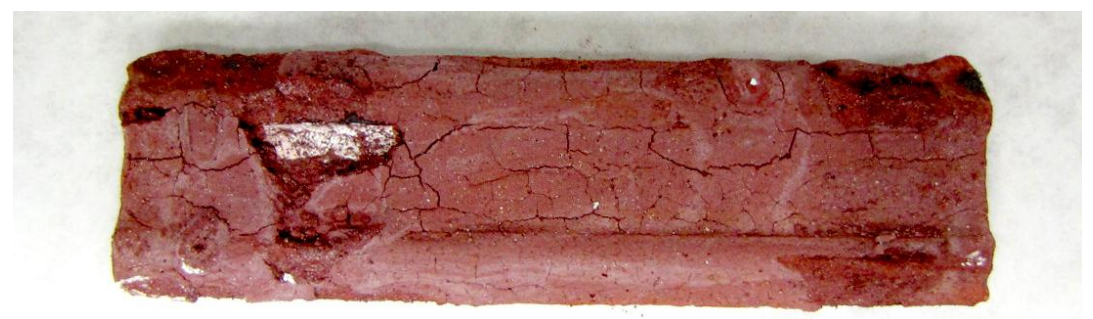

\section{ID Surface}
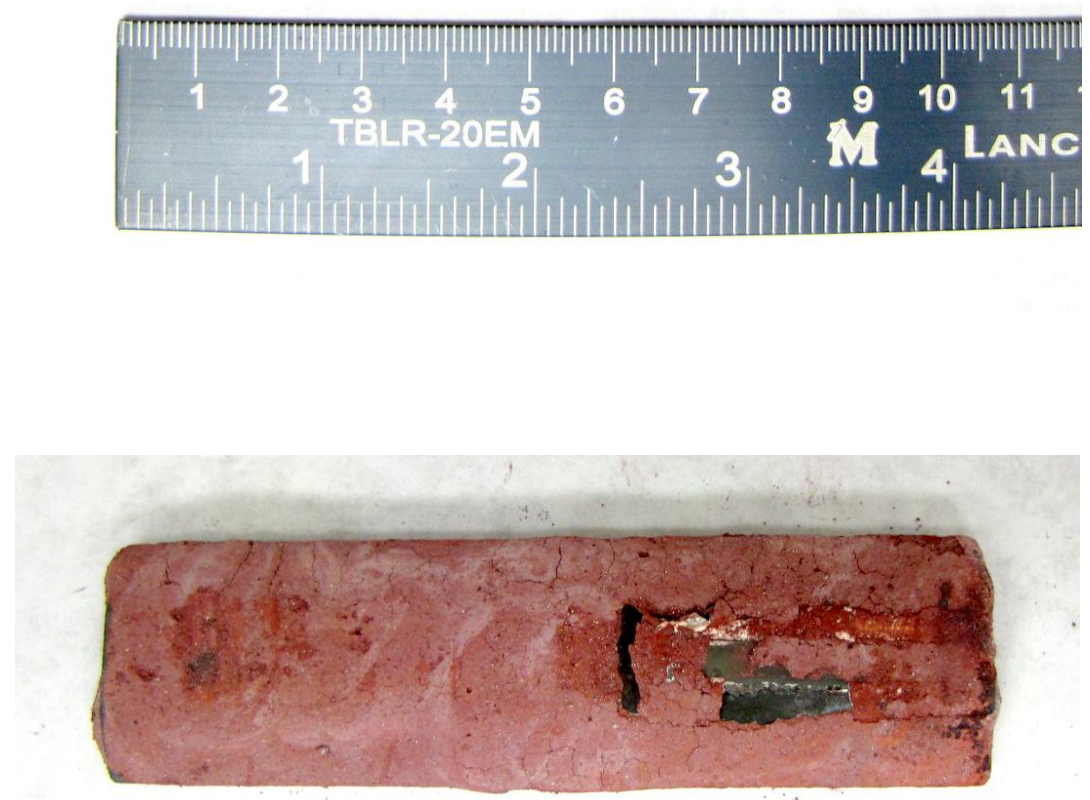

OD Surface

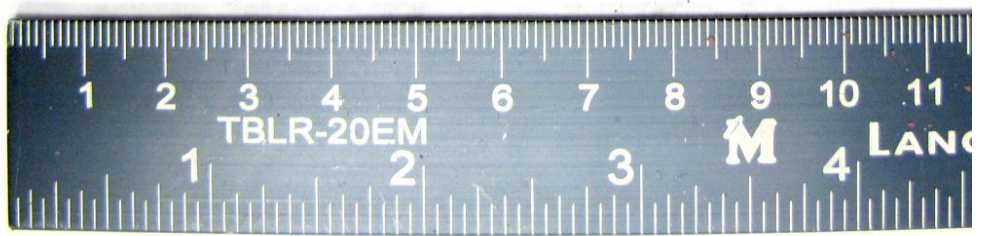

Figure 5. The typical views of the test specimen (with the ash deposit) after the corrosion testing at $600^{\circ} \mathrm{C}$ are shown. 


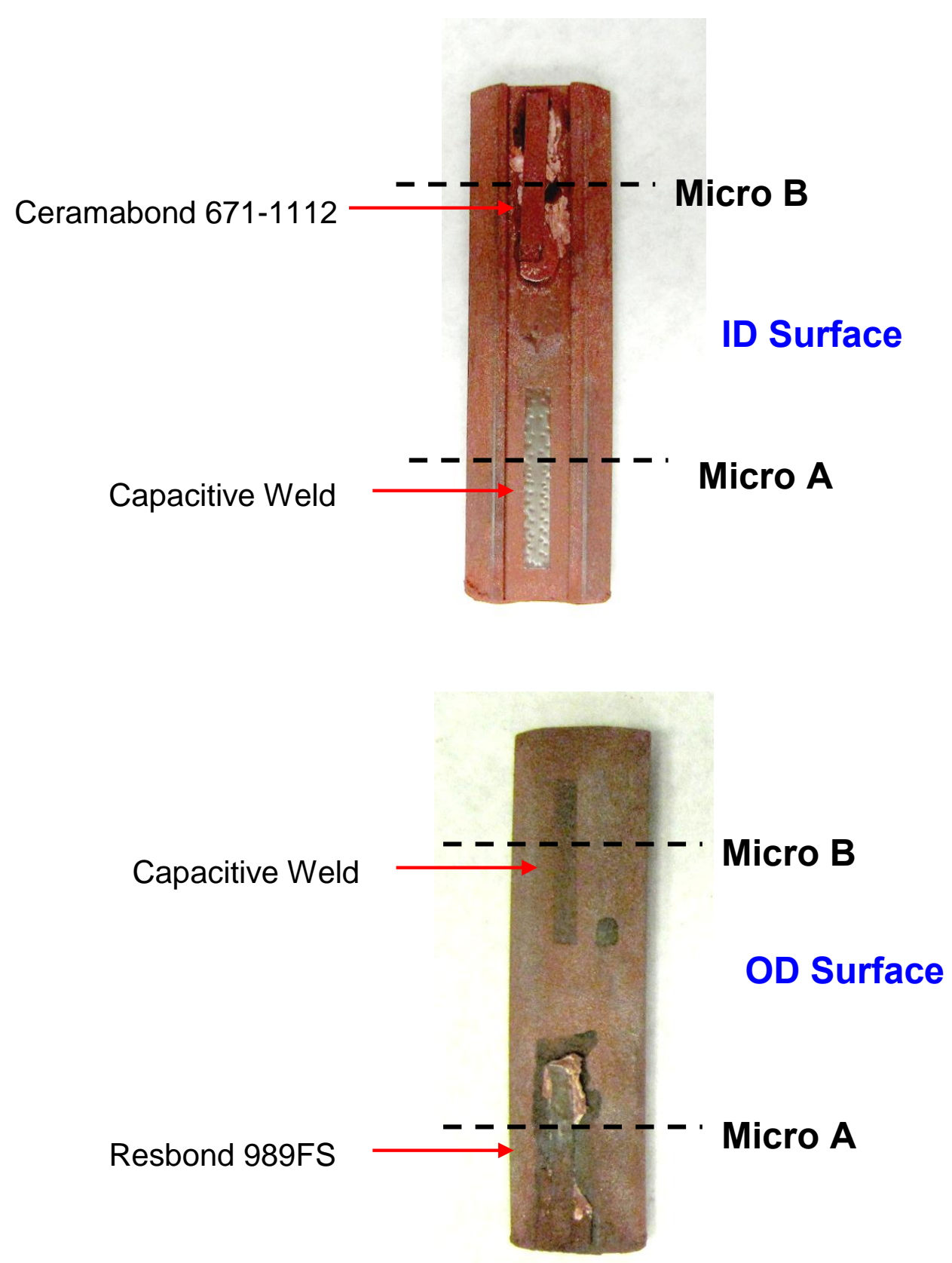

Figure 6. The typical views of the test specimen (after cleaning the ash deposit) after the corrosion testing at $600^{\circ} \mathrm{C}$ are shown. As seen, the Inconel strips got detached from the substrate where Ceramabond 671-1112 and Resbond 989FS were used. The approximate locations from where the metallographic sections were taken are indicated in this Figure. 

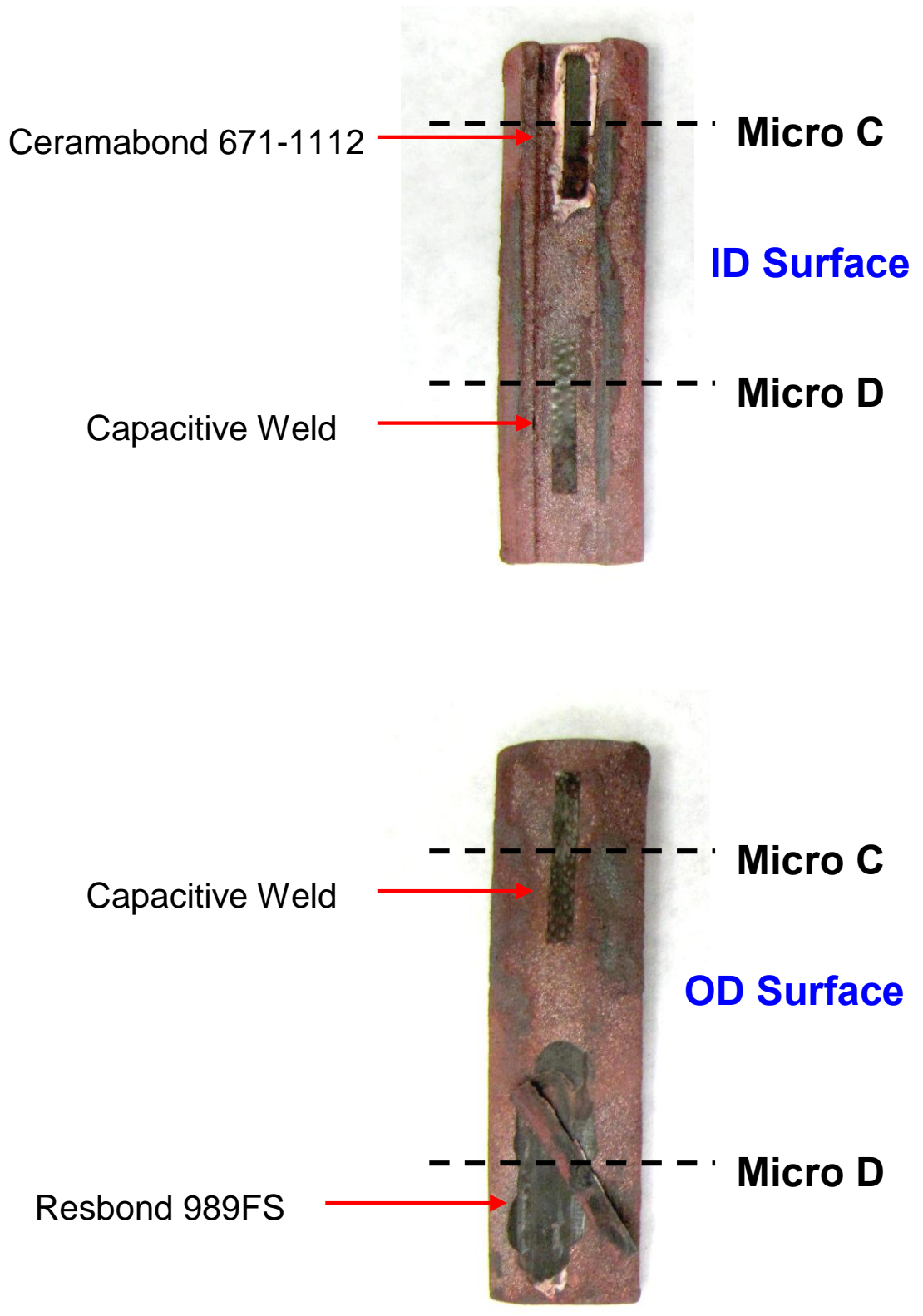

Figure 7. The typical views of the test specimen (after cleaning the ash deposit) after the corrosion testing at $650^{\circ} \mathrm{C}$ are shown. As seen, the Inconel strips got detached from the substrate where Ceramabond 671-1112 and Resbond 989FS were used. The approximate locations from where the metallographic sections were taken are indicated in this Figure. 


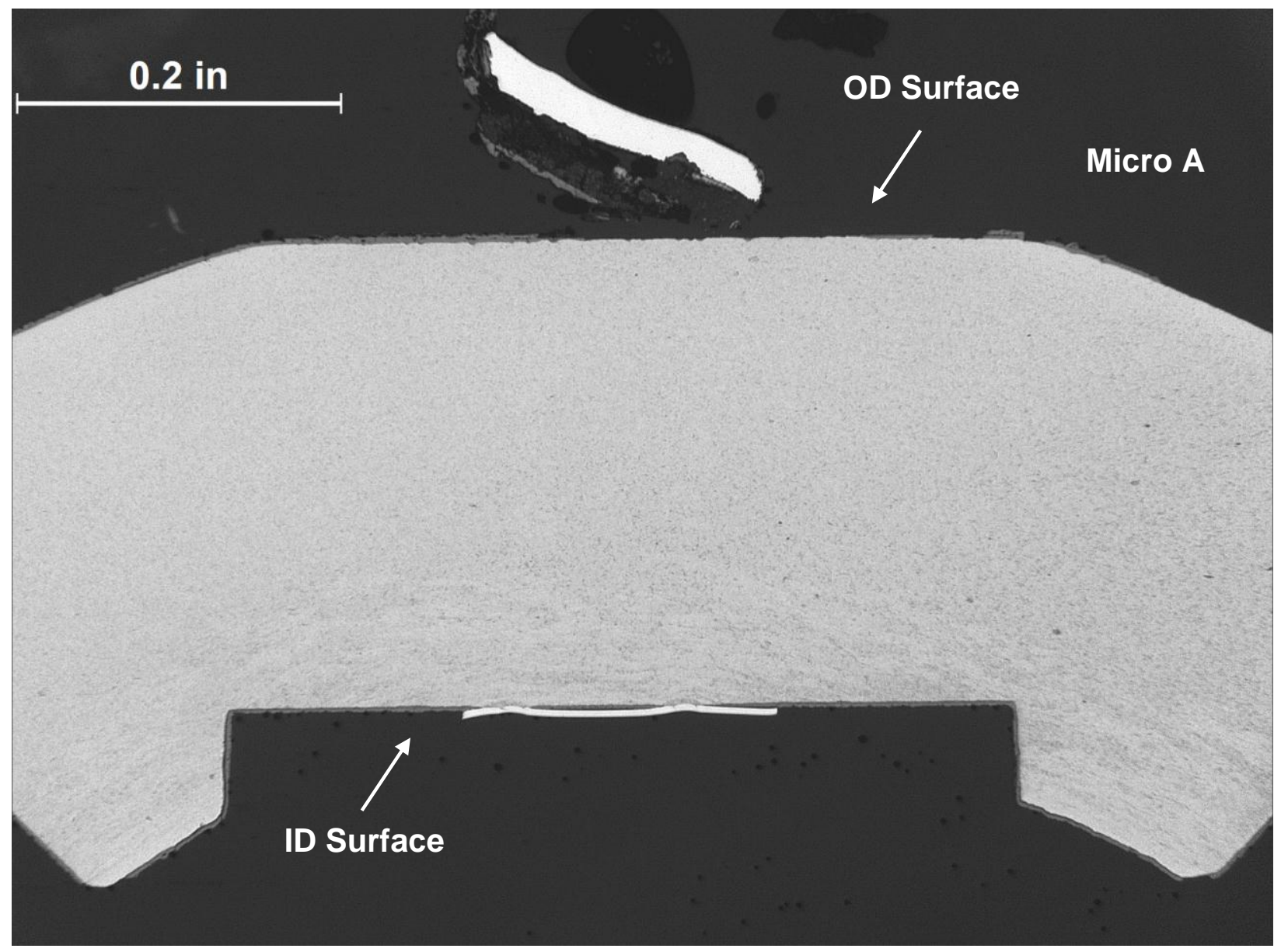

Figure 8. Documenting the overall condition of Micro $A$ after the corrosion testing. As shown, the Inconel strip is intact with the substrate on the ID surface where capacitive weld attachment was used. The Inconel strip was detached from the substrate on the OD surface where Resbond 989FS was used. 


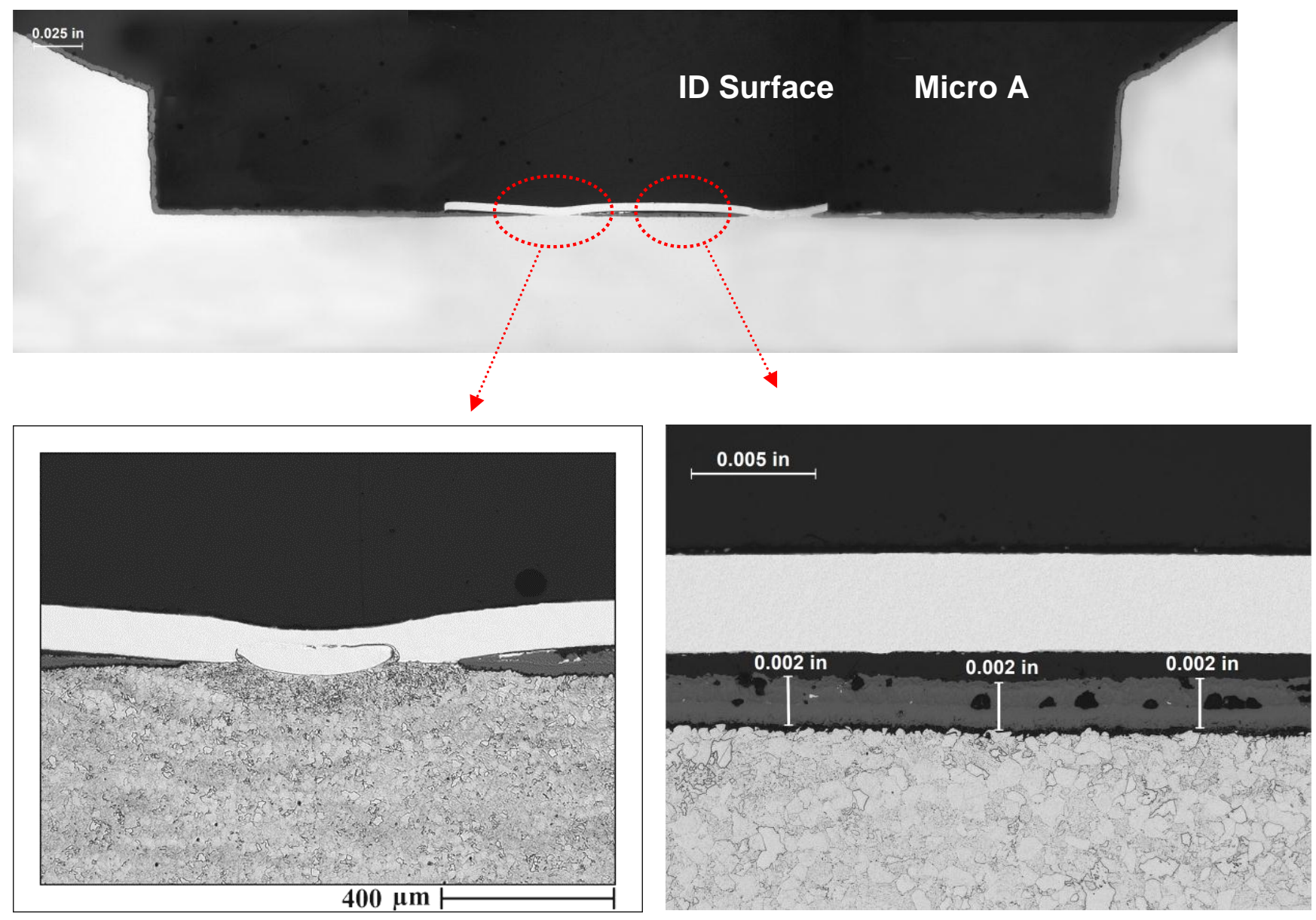

Figure 9. Documenting the condition of the test specimen after corrosion testing at $600^{\circ} \mathrm{C}$ on the ID surface at a location where capacitive weld was used to attach the Inconel strip. As seen, the attachment was judged to be satisfactory; however, there was corrosion product ( 2 mils thick) seen underneath the Inconel strip and that may pose an issue in terms of durability. 

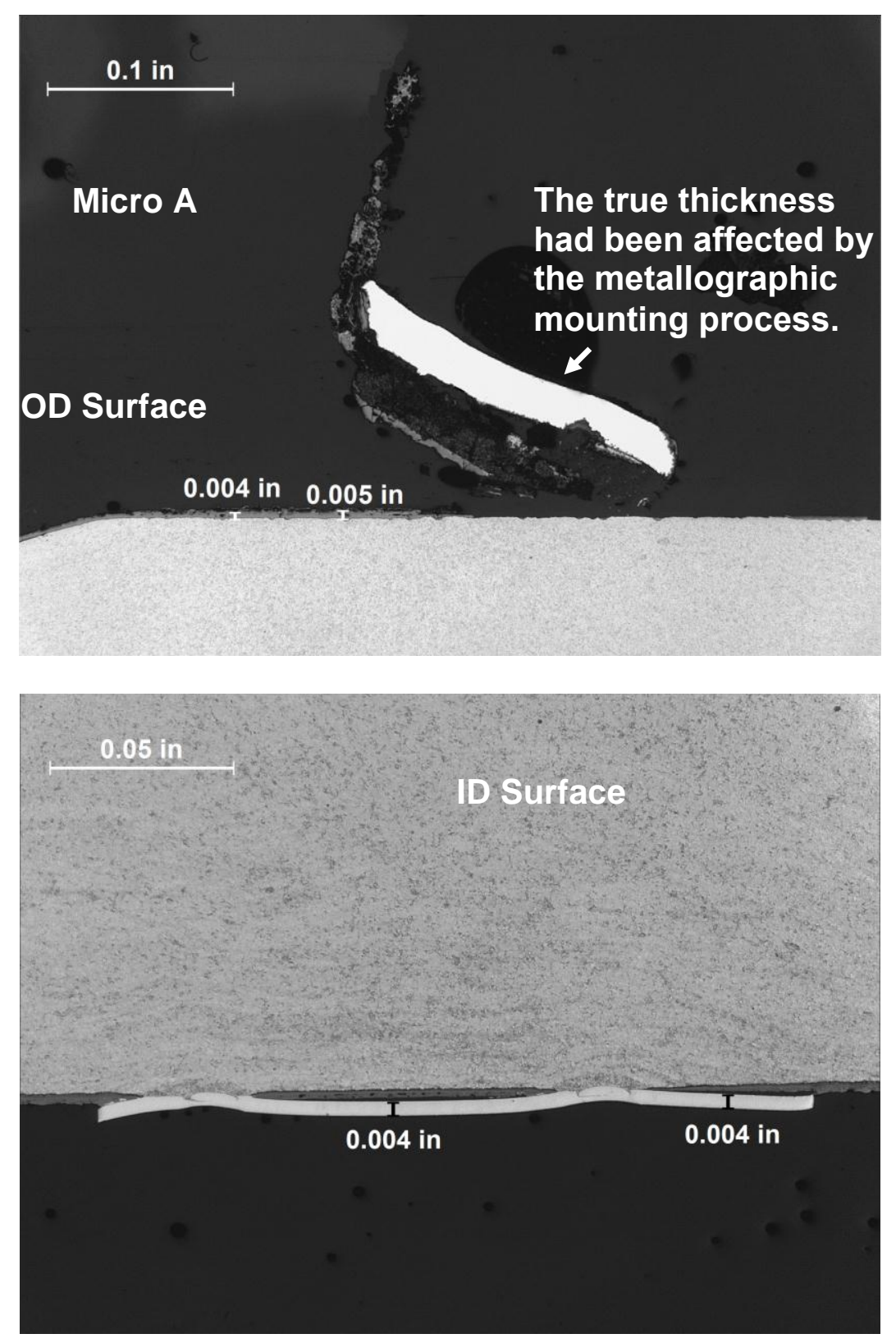

Figure 10. Documenting the Inconel strip thickness profiles on the OD and ID surfaces after the corrosion test. The thickness of the Inconel strip is $\sim 4$ mils. 


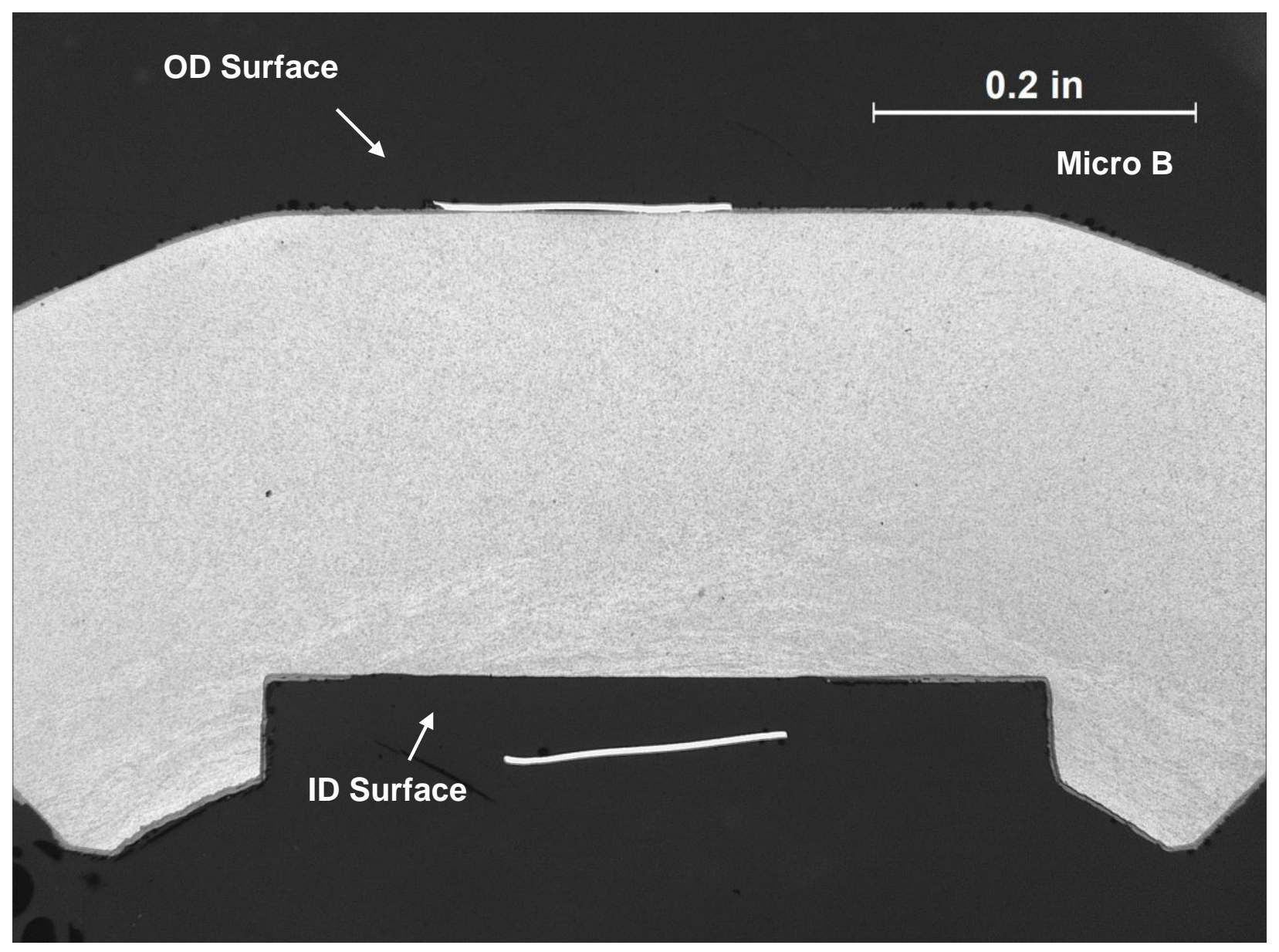

Figure 11. Documenting the overall condition of Micro B after the corrosion testing. As shown, the Inconel strip is intact with the substrate on the OD surface where capacitive weld attachment was used. The Inconel strip was detached from the substrate on the ID surface where Ceramabond 671-1112 was used. 


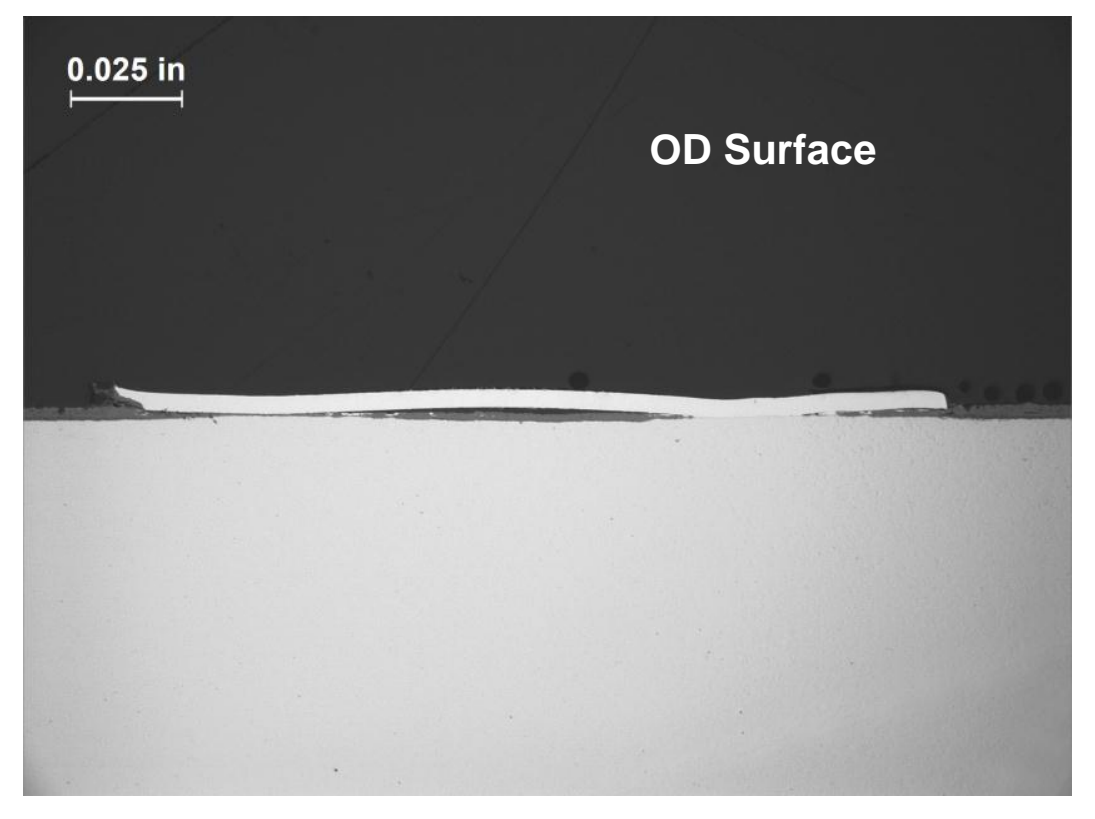

\section{Micro B}
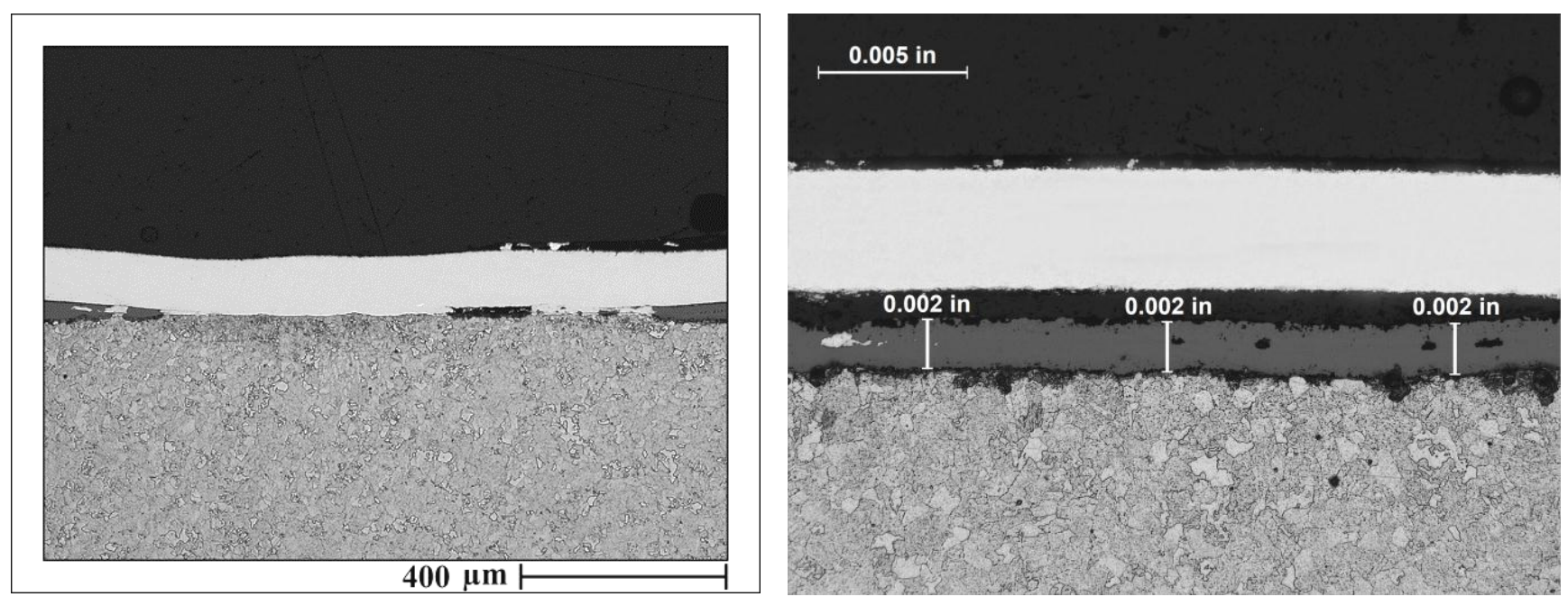

Figure 12. Documenting the condition of the test specimen after corrosion testing at $600^{\circ} \mathrm{C}$ on the OD surface at a location where capacitive weld was used to attach the Inconel strip. The weld attachment was judged to be satisfactory; however, there was corrosion product ( 2 mils thick) seen underneath the Inconel strip and that may pose an issue in terms of durability. 


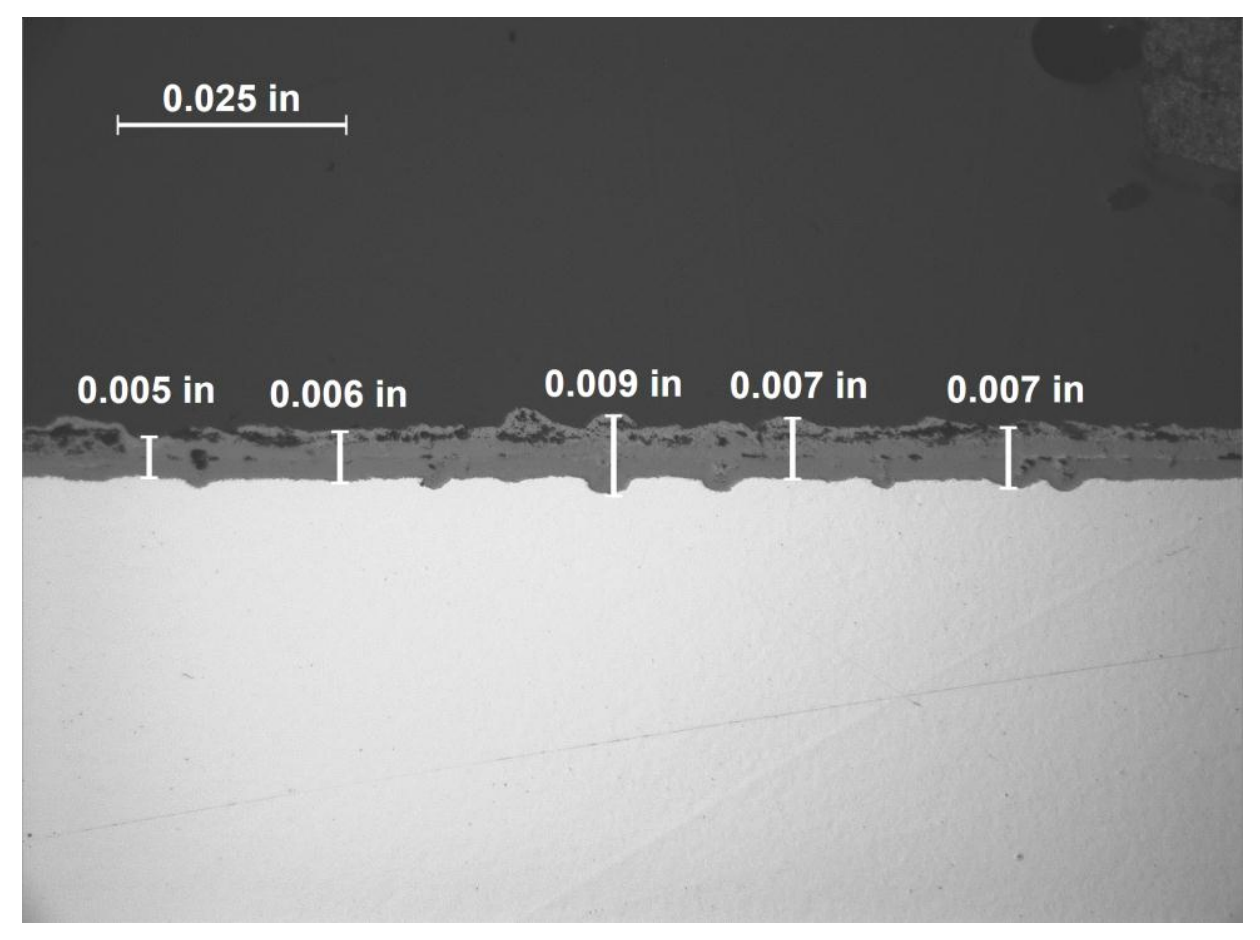

Figure 13. Documenting the corrosion product thickness observed on the test specimen after corrosion testing at $600^{\circ} \mathrm{C}$. The maximum corrosion product thickness observed was $\sim 9$ mils. 


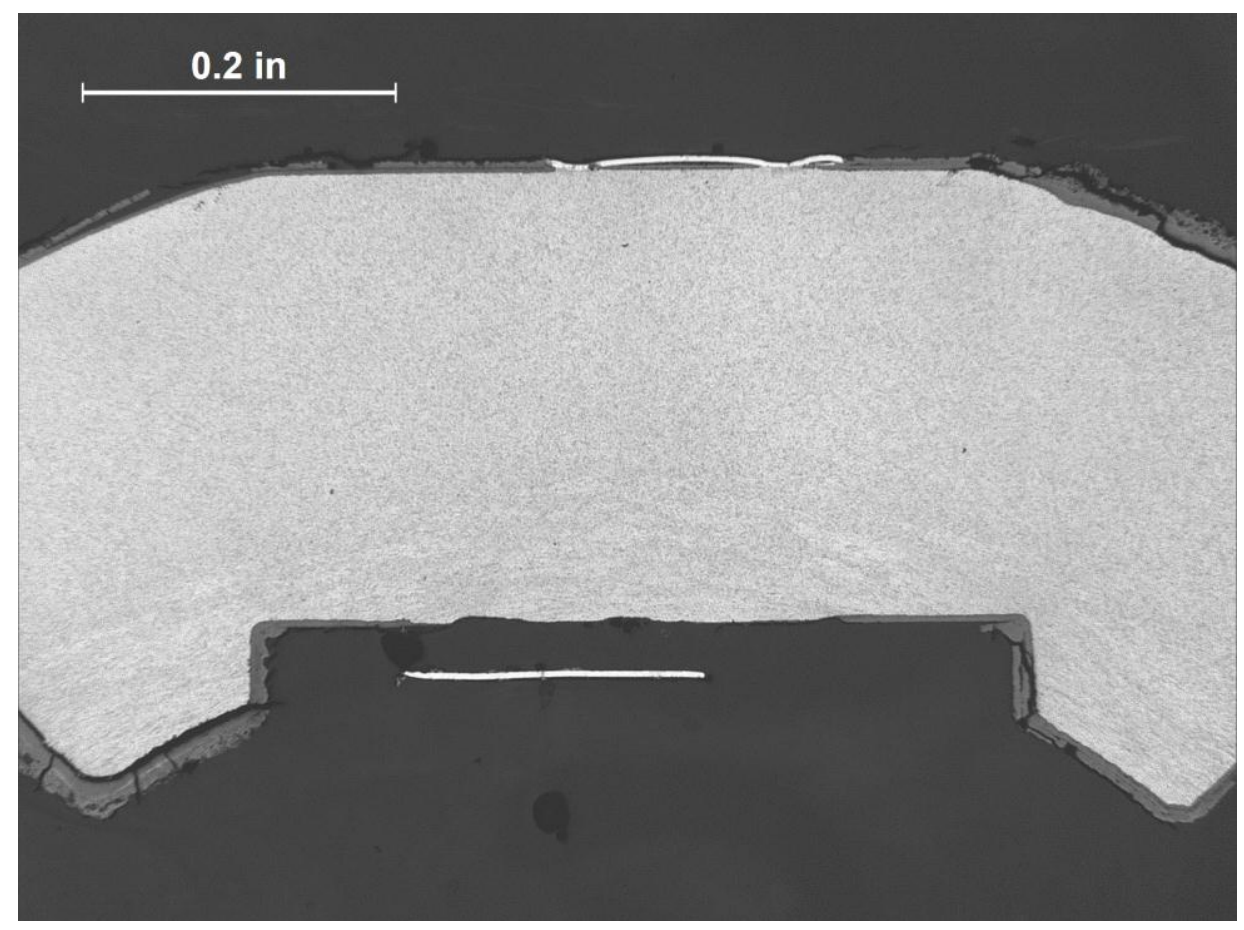

Micro C

Figure 14. Documenting the overall condition of the test specimen on Micro $\mathrm{C}$ location after the corrosion testing at $650^{\circ} \mathrm{C}$. As shown, the Inconel strip is intact with the substrate on the OD surface where capacitive weld attachment was used. The Inconel strip was detached from the substrate on the ID surface where Ceramabond 671-1112 was used. 


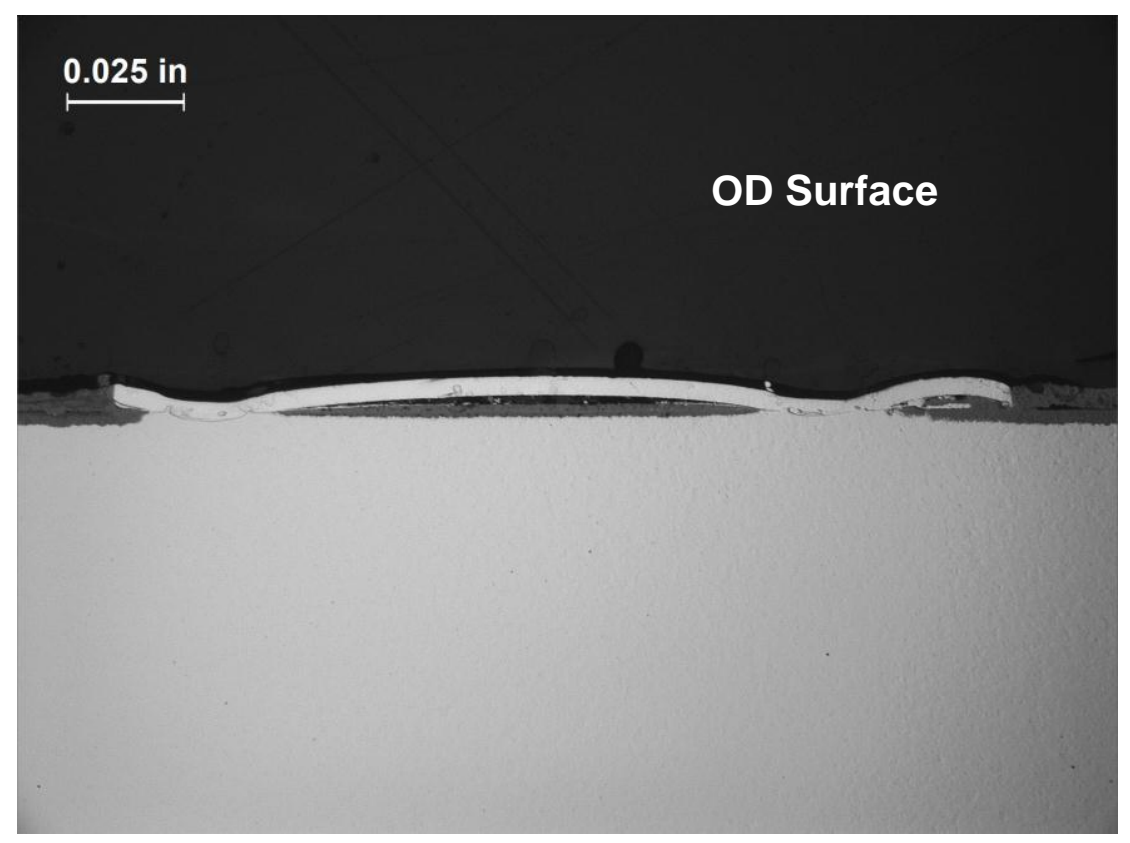

\section{Micro C}
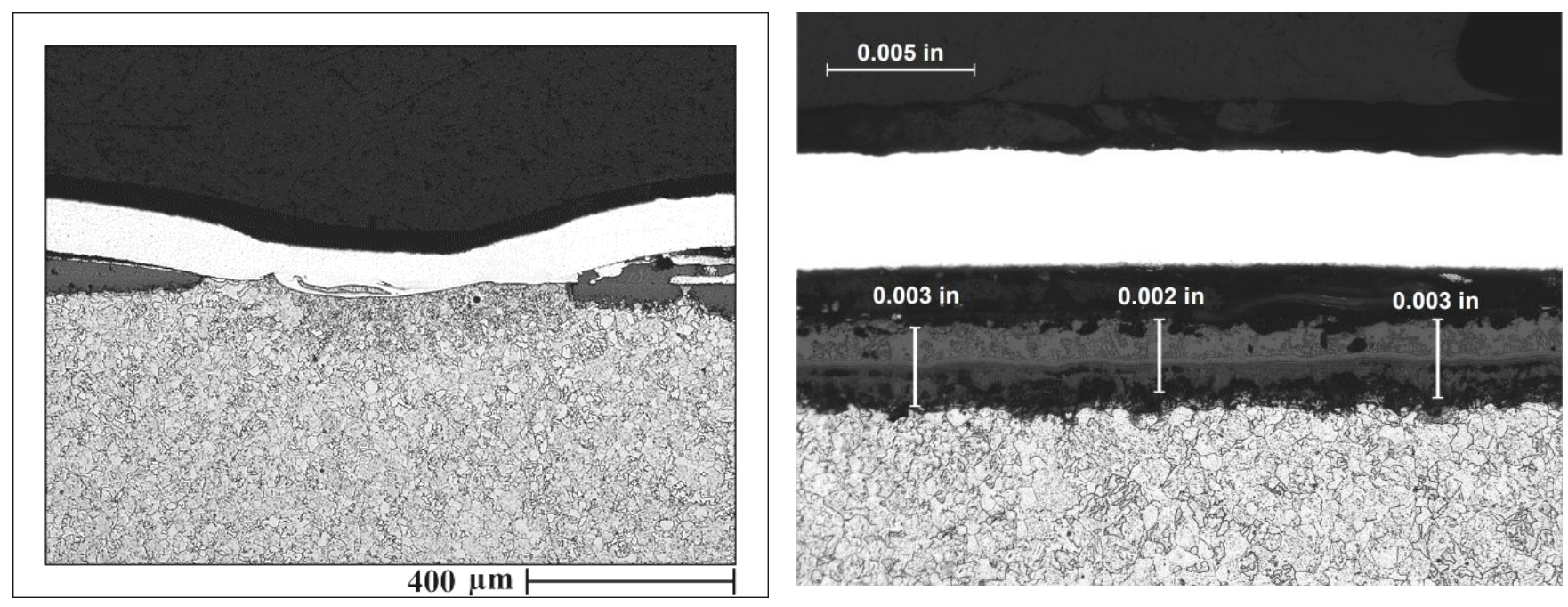

Figure 15. Documenting the condition of the test specimen after corrosion testing at $650^{\circ} \mathrm{C}$ on the $\mathrm{OD}$ surface at a location where capacitive weld was used to attach the Inconel strip. The weld attachment was judged to be satisfactory; however, there was corrosion product ( 2-3 mils thick) seen underneath the Inconel strip and that may pose an issue in terms of durability. 


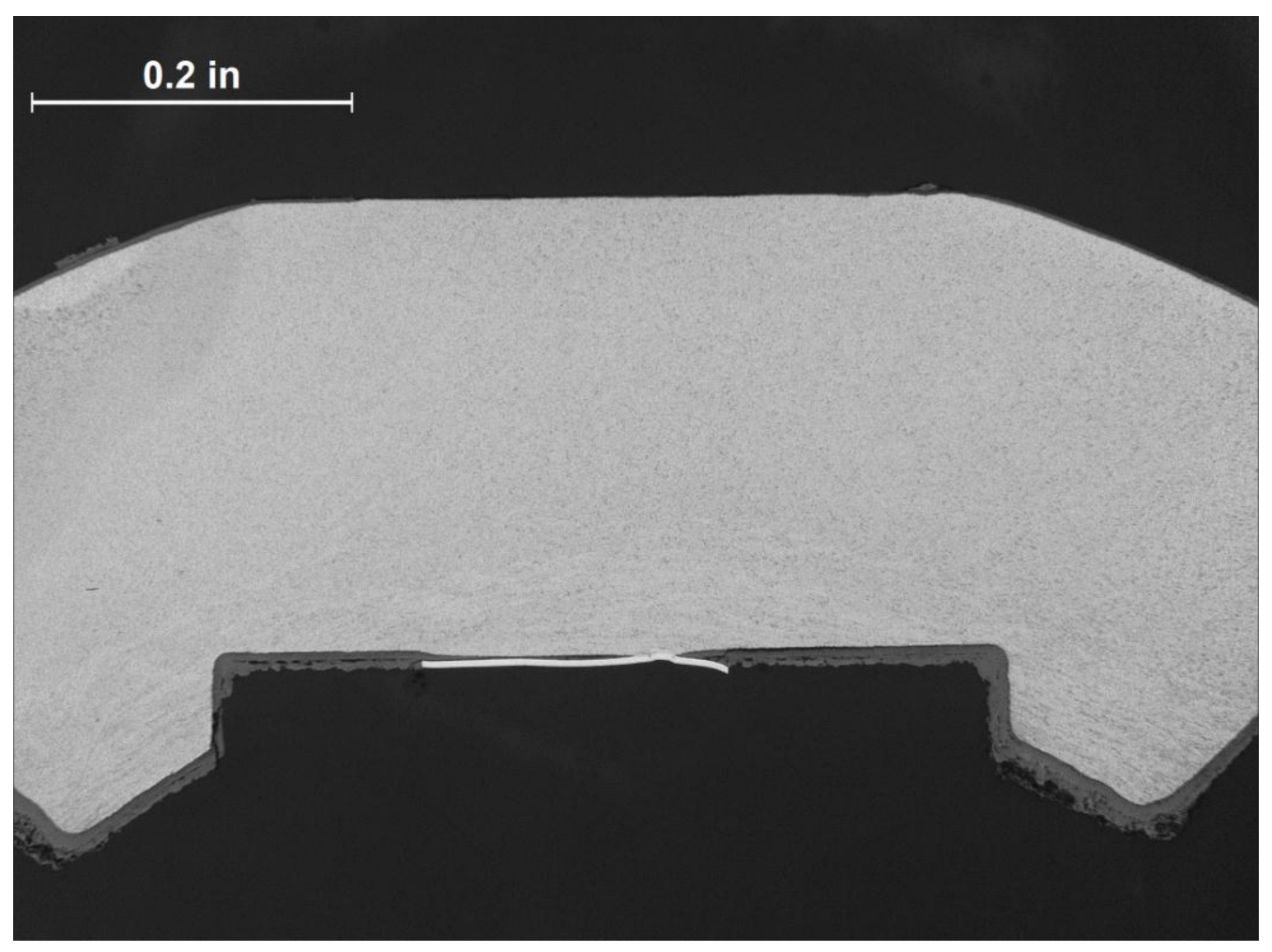

Micro D

Figure 16. Documenting the overall condition of Micro $D$ after the corrosion testing. As shown, the Inconel strip is intact with the substrate on the ID surface where capacitive weld attachment was used. The Inconel strip was detached from the substrate on the OD surface where Resbond 989FS was used. 


\section{ID Surface}

Micro D

\subsection{5 in}
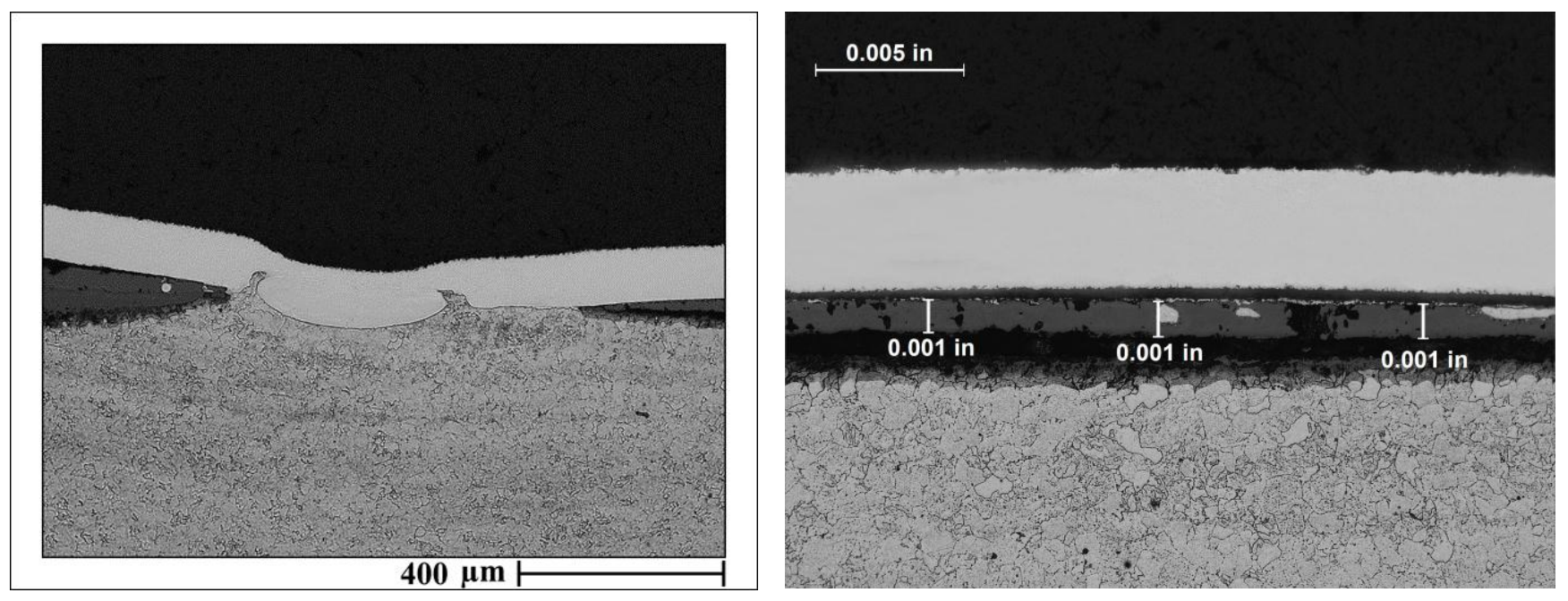

Figure 17. Documenting the condition of the test specimen after corrosion testing at $650^{\circ} \mathrm{C}$ on the ID surface at a location where capacitive weld was used to attach the Inconel strip. As seen, the attachment was judged to be satisfactory; however, there was corrosion product ( 1 mil thick) seen underneath the Inconel strip and that may pose an issue in terms of durability. 


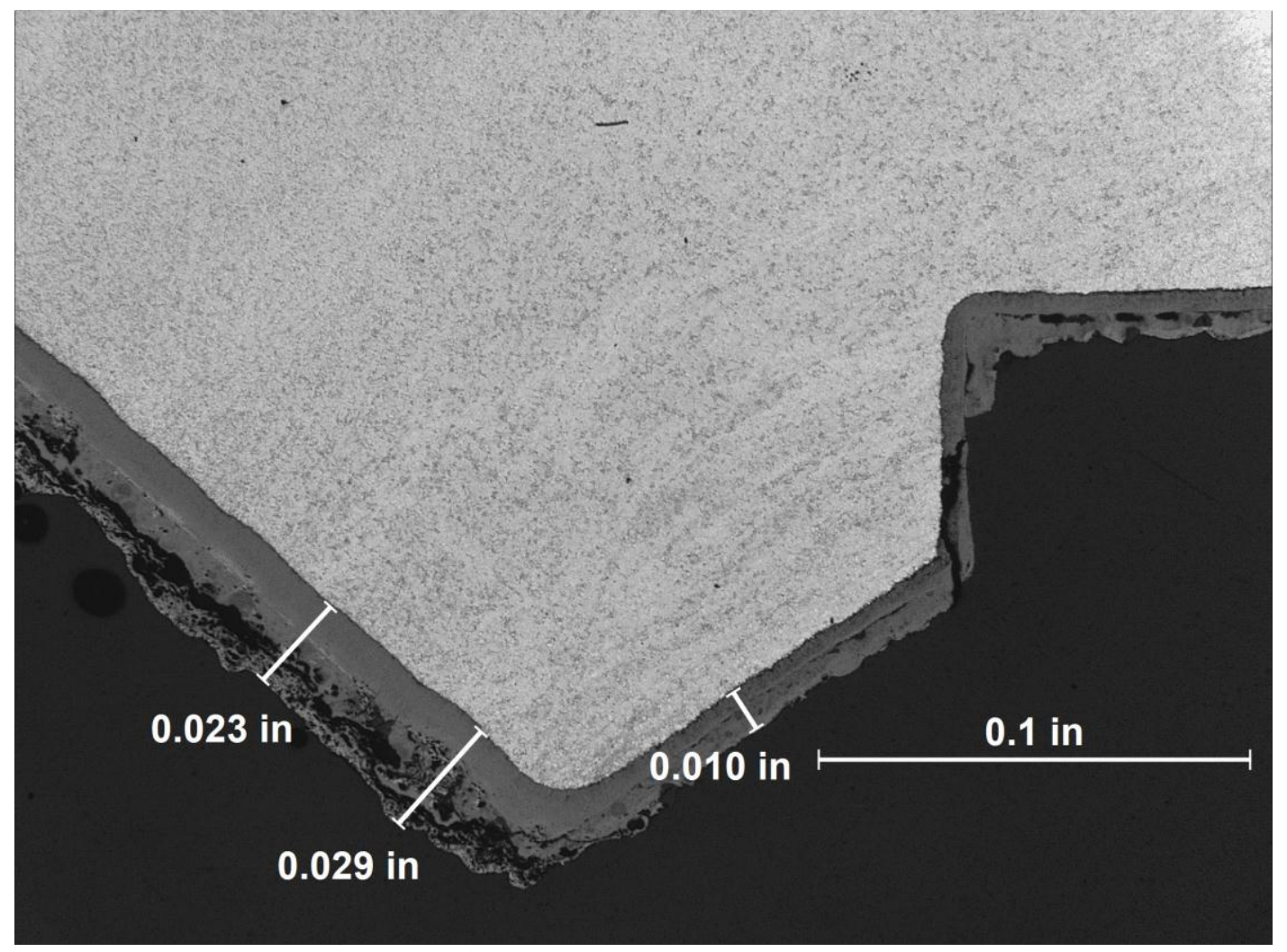

Figure 18. Documenting the corrosion product thickness observed on the test specimen after corrosion testing at $650^{\circ} \mathrm{C}$. The maximum corrosion product thickness observed was $\sim 29$ mils. 


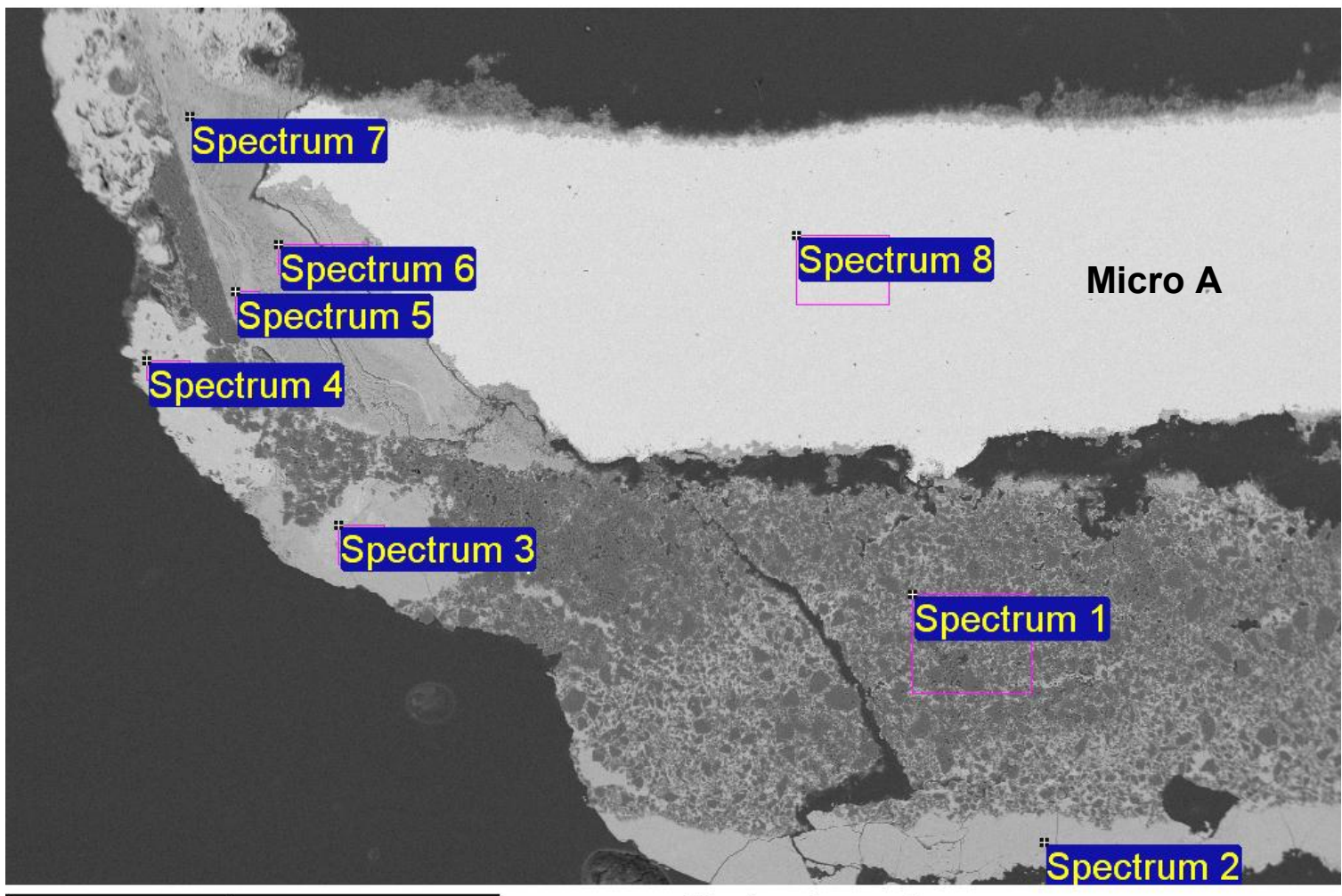

$1 \mathrm{~mm}$

Electron Image 1

Semi-Quantitative Analysis, Wt\%

\begin{tabular}{|c|c|c|c|c|c|c|c|c|}
\hline Spectrum & $\mathrm{O}$ & $\mathrm{Al}$ & $\mathrm{Si}$ & $\mathrm{S}$ & $\mathrm{Ti}$ & $\mathrm{Cr}$ & $\mathrm{Fe}$ & $\mathrm{Ni}$ \\
\hline & & & & & & & & \\
\hline Spectrum 1 & 20.72 & 46.36 & 1.45 & & & & 31.47 & \\
\hline Spectrum 2 & 8.60 & & & & & 4.09 & 87.31 & \\
\hline Spectrum 3 & 9.73 & & & & & & 73.92 & 16.35 \\
\hline Spectrum 4 & 5.48 & & & 9.79 & & & 0.52 & 84.21 \\
\hline Spectrum 5 & 10.38 & & & 3.59 & 0.60 & 36.16 & 19.11 & 30.17 \\
\hline Spectrum 6 & 10.22 & 1.21 & & 3.58 & 0.57 & 39.29 & 15.49 & 29.64 \\
\hline Spectrum 7 & 15.96 & 1.31 & & & 0.66 & 33.02 & 35.69 & 13.36 \\
\hline Spectrum 8 & & & & & & 15.92 & 8.58 & 75.50 \\
\hline
\end{tabular}

Figure 19. Shown are the SEM-EDS results of the corrosion product that was found along the edge of the Inconel strip, which was detached from the substrate on the OD surface where Resbond 989FS was used. The corrosion mechanism observed was predominantly oxidation and sulfidation. The composition of the Inconel strip was consistent with the Inconel-600 type material. 


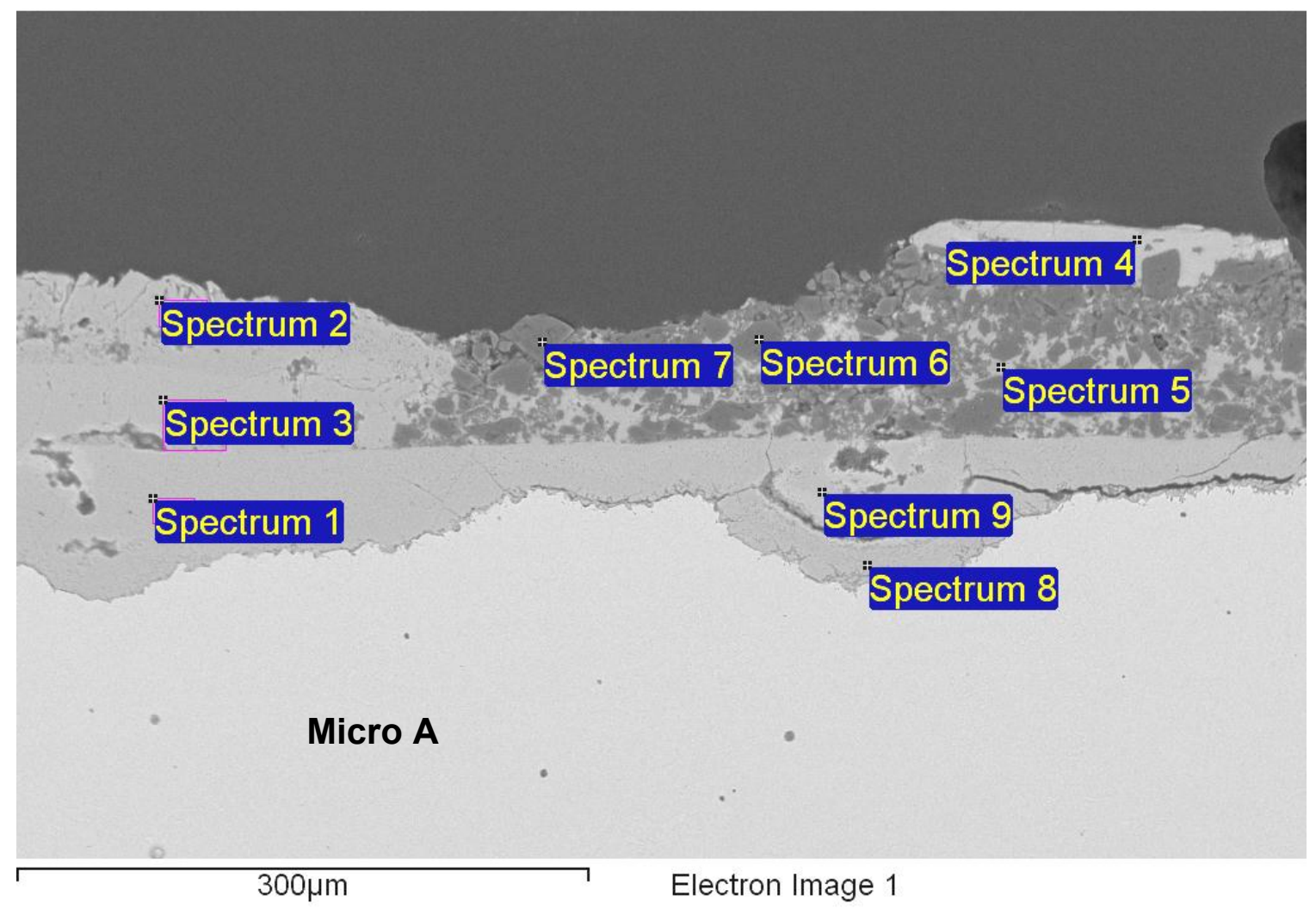

Semi-Quantitative Analysis, Wt\%

\begin{tabular}{|c|c|c|c|c|c|c|c|}
\hline Spectrum & $\mathrm{O}$ & $\mathrm{Al}$ & $\mathrm{S}$ & $\mathrm{Cr}$ & $\mathrm{Mn}$ & $\mathrm{Fe}$ & $\mathrm{Ni}$ \\
\hline & & & & & & & \\
\hline Spectrum 1 & 8.48 & & & 4.63 & & 86.88 & \\
\hline Spectrum 2 & 9.15 & & & & & 90.85 & \\
\hline Spectrum 3 & 8.96 & & 2.80 & & & 88.24 & \\
\hline Spectrum 4 & 5.92 & & 1.96 & & & 63.44 & 28.68 \\
\hline Spectrum 5 & 9.40 & 4.72 & & & & 85.89 & \\
\hline Spectrum 6 & 32.85 & 67.15 & & & & & \\
\hline Spectrum 7 & 29.51 & 70.49 & & & & & \\
\hline Spectrum 8 & 9.12 & & & 4.00 & & 86.88 & \\
\hline Spectrum 9 & 9.36 & & & 3.29 & 0.83 & 86.51 & \\
\hline & & & & & & & \\
\hline
\end{tabular}

Figure 20. Shown are the SEM-EDS results of the corrosion product that was observed along the OD surface of the test specimen where the Inconel strip was detached from the substrate (Micro A). Some remnants of the corrosion product from the Inconel strip were also observed on the outer most layer (Spectrum 4). 


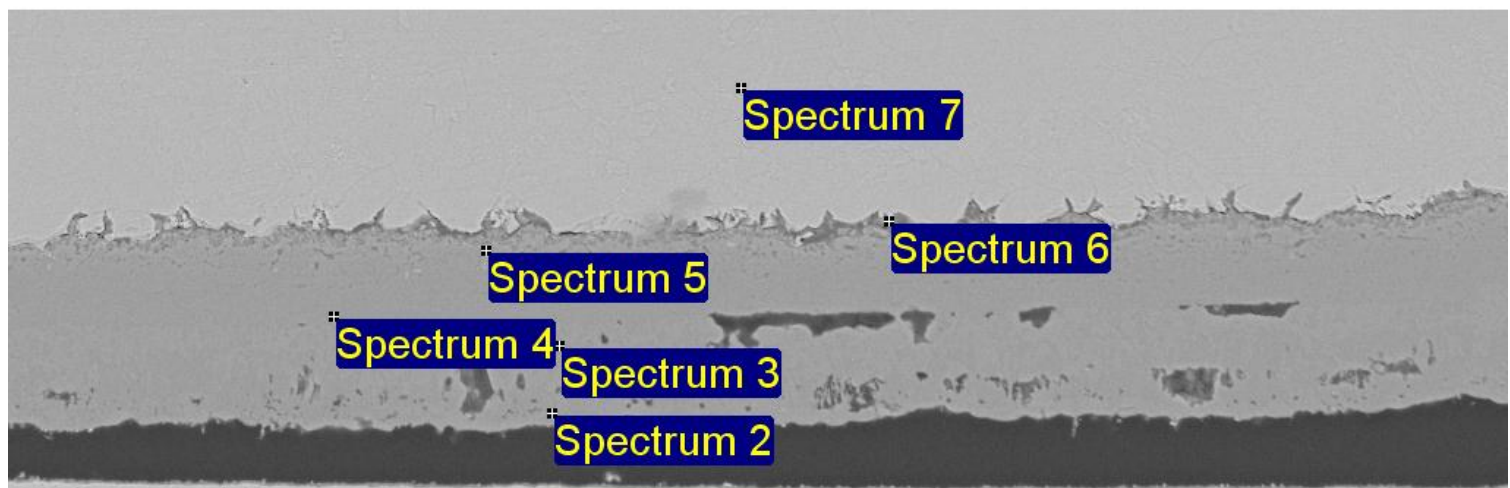

Micro A

Spectrum 1

$200 \mu \mathrm{m}$

Electron Image 1

Semi-Quantitative Analysis, Wt\%

\begin{tabular}{|c|c|c|c|c|c|}
\hline Spectrum & $\mathrm{O}$ & $\mathrm{Cr}$ & $\mathrm{Mn}$ & $\mathrm{Fe}$ & $\mathrm{Ni}$ \\
\hline & & & & & \\
\hline Spectrum 1 & & 15.80 & & 9.08 & 75.12 \\
\hline Spectrum 2 & 7.38 & & & 92.62 & \\
\hline Spectrum 3 & 7.54 & & 0.64 & 91.82 & \\
\hline Spectrum 4 & 7.90 & 4.06 & & 88.04 & \\
\hline Spectrum 5 & 6.72 & 3.68 & 0.80 & 88.79 & \\
\hline Spectrum 6 & 3.77 & 3.31 & 1.07 & 91.85 & \\
\hline Spectrum 7 & & 2.79 & 1.07 & 96.15 & \\
\hline & & & & & \\
\hline
\end{tabular}

Figure 21. Shown are the SEM-EDS results of the corrosion product that was observed along the ID surface underneath the Inconel strip from Micro A. The composition of the Inconel strip was consistent with the Inconel-600 (Spectrum 1). The substrate composition was consistent with the T22 material (Spectrum 7). 